\title{
Combining a cochlear implant and a hearing aid in opposite ears
}

Citation for published version (APA):

Devocht, E. (2019). Combining a cochlear implant and a hearing aid in opposite ears: The best of both worlds. [Doctoral Thesis, Maastricht University]. Maastricht University.

https://doi.org/10.26481/dis.20190703ed

Document status and date:

Published: 01/01/2019

DOI:

10.26481/dis.20190703ed

Document Version:

Publisher's PDF, also known as Version of record

\section{Please check the document version of this publication:}

- A submitted manuscript is the version of the article upon submission and before peer-review. There can be important differences between the submitted version and the official published version of record.

People interested in the research are advised to contact the author for the final version of the publication, or visit the DOI to the publisher's website.

- The final author version and the galley proof are versions of the publication after peer review.

- The final published version features the final layout of the paper including the volume, issue and page numbers.

Link to publication

\footnotetext{
General rights rights.

- You may freely distribute the URL identifying the publication in the public portal. please follow below link for the End User Agreement:

www.umlib.nl/taverne-license

Take down policy

If you believe that this document breaches copyright please contact us at:

repository@maastrichtuniversity.nl

providing details and we will investigate your claim.
}

Copyright and moral rights for the publications made accessible in the public portal are retained by the authors and/or other copyright owners and it is a condition of accessing publications that users recognise and abide by the legal requirements associated with these

- Users may download and print one copy of any publication from the public portal for the purpose of private study or research.

- You may not further distribute the material or use it for any profit-making activity or commercial gain

If the publication is distributed under the terms of Article $25 \mathrm{fa}$ of the Dutch Copyright Act, indicated by the "Taverne" license above, 


\section{COMBINING A COCHLEAR IMPLANT AND A HEARING AID NOPPOSTEEARS}

THE BEST OF BOTH WORLDS

Elke Devocht
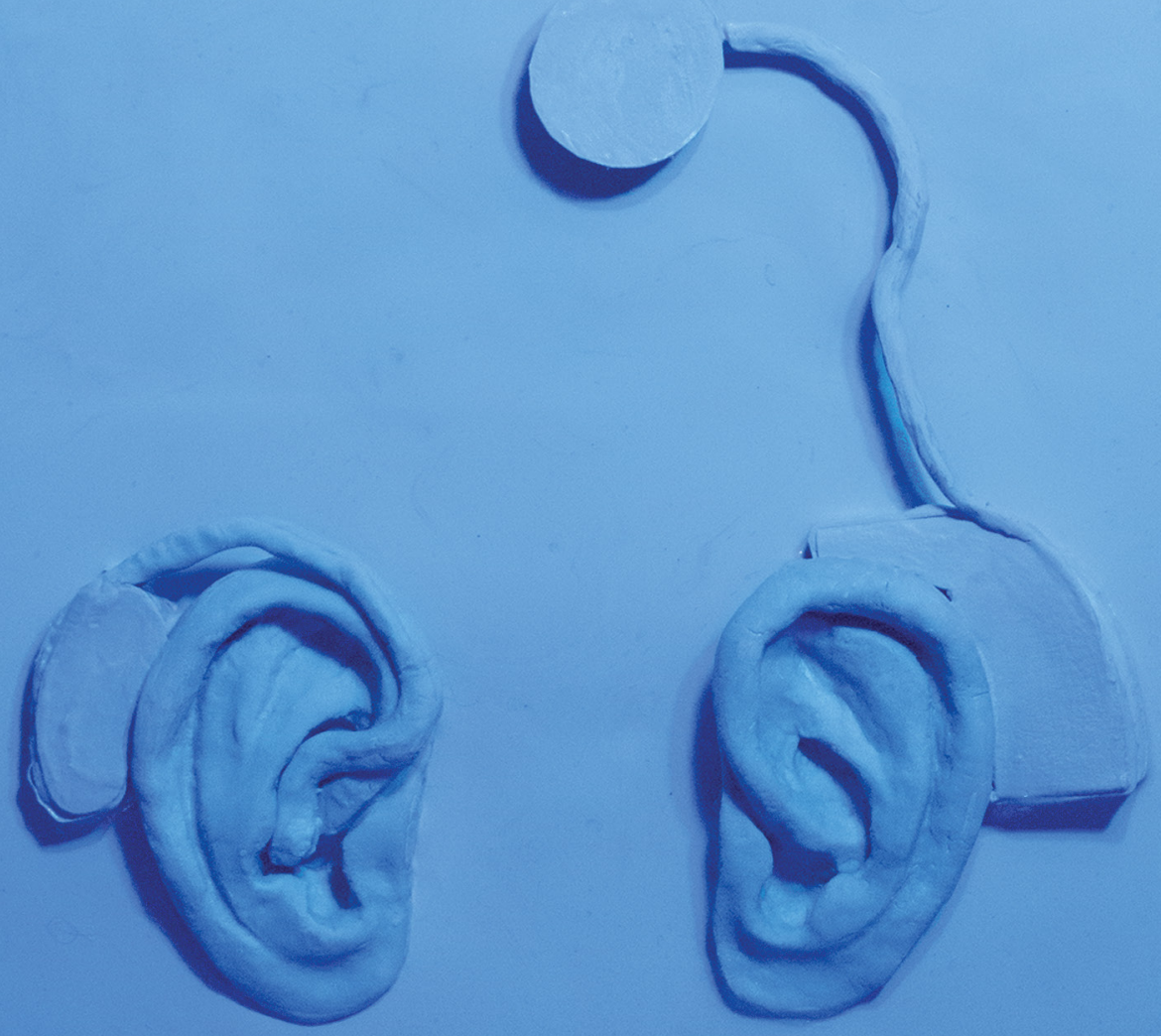



\section{COMBINING A COCHLEAR IMPLANT AND A HEARING AID IN OPPOSITE EARS \\ THE BEST OF BOTH WORLDS}

\section{EEN COCHLEAIR IMPLANTAAT IN HET ENE OOR EN EEN HOORTOESTEL IN HET ANDERE OOR HET BESTE VAN BEIDE WERELDEN COMBINEREN}


(C) Copyright Elke Devocht, Maastricht 2019

ISBN: 978-94-6380-343-4

Cover design by Rosse \& Josse ; thisisjoren.com

Layout by Elke Devocht; Anouk Linssen

Printed by www.proefschriftmaken.nl 


\section{COMBINING A COCHLEAR IMPLANT AND A HEARING AID IN OPPOSITE EARS THE BEST OF BOTH WORLDS}

\section{PROEFSCHRIFT}

ter verkrijging van de graad van doctor aan de Universiteit Maastricht, op gezag van de Rector Magnificus, Prof. dr. Rianne M. Letschert, volgens het besluit van het College van Decanen, in het openbaar te verdedigen op woensdag 3 juli 2019 om 10 uur

door

Elke M. J. Devocht 


\section{PROMOTOR}

Prof. Dr. H. Kingma

\section{COPROMOTOR}

Dr. E. L J. George

\section{BEOORDELINGSCOMMISSIE}

Prof. Dr. B. Kremer (voorzitter)

Prof. Dr. T. Francart (Katholieke Universiteit Leuven, België)

Prof. Dr. E. Formisano

Prof. Dr. M. A. Joore

Prof. Dr. S. E. Kramer (Amsterdam Universitair Medische Centra, locatie VUmc)

The research described in this thesis was performed at the School for Mental Health and Neuroscience of the Faculty of Health, Medicine and Life Sciences of Maastricht University (UM) and the Department of Otorhinolaryngology, Head and Neck Surgery of the Maastricht University Medical Centre (MUMC+). The work of the first author was financially supported by a research grant from Advanced Bionics Inc. 


\section{CONTENTS}

GENERAL INTRODUCTION . . .

CHAPTER 1

Bimodal hearing aid retention

after unilateral cochlear implantation

15

CHAPTER 2

Self-assessment of unilateral and bimodal

cochlear implant experiences in daily life

CHAPTER 3

The benefits of bimodal aiding on extended dimensions of speech perception: Intelligibility, listening effort, and sound quality 65

CHAPTER 4

Monaural beamforming in bimodal cochlear implant users:

Effect of (a)symmetric directivity and noise type

CHAPTER 5

Revisiting place-pitch match in CI recipients

using 3D imaging analysis

GENERAL DISCUSSION AND VALORISATION 133

SUMMARY 143

SAMENVATTING 149

REFERENCES 155

DANKWOORD 175

ABOUT THE AUTHOR[S]. 181 



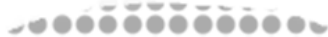

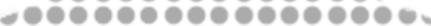

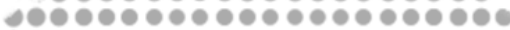

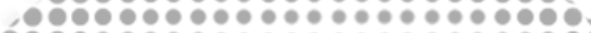

,

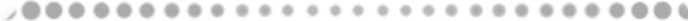

,

우



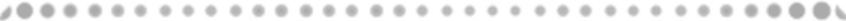

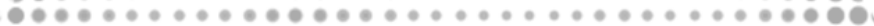

○.

우…

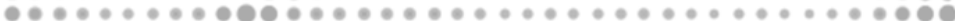

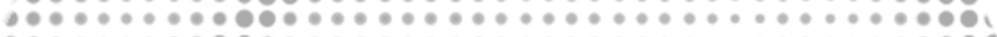

눈

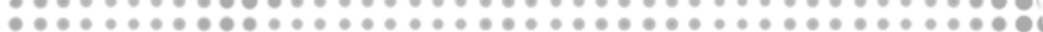

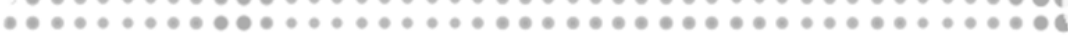

.

,

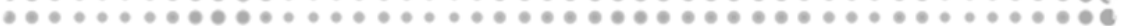

,

두우

(a)

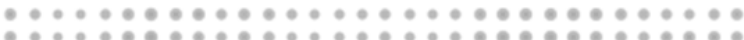

순.

.

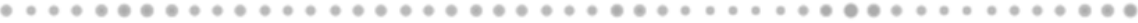

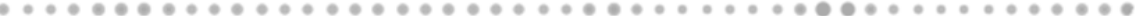

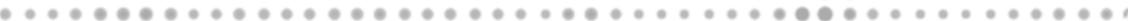

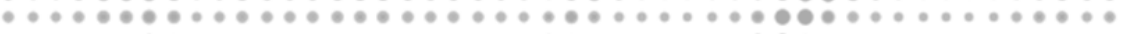

,

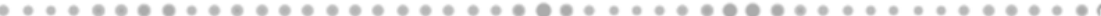

ㄷ․

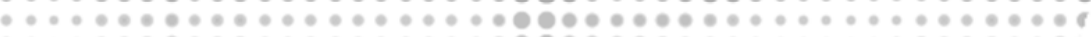

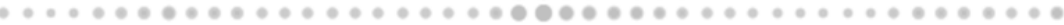

"

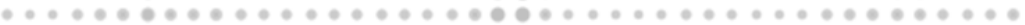

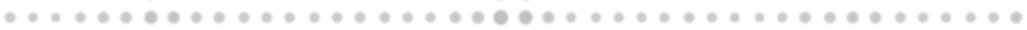

눙ำ

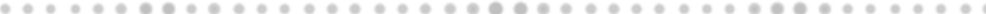

, + , +

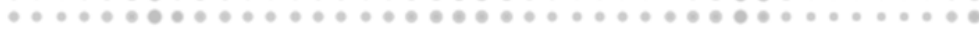

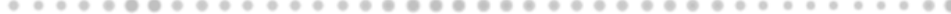

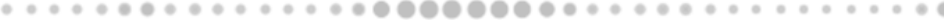

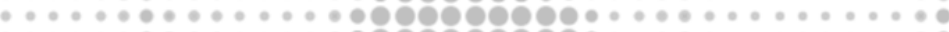

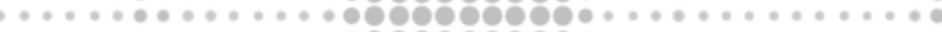

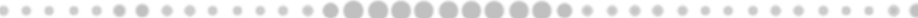

เ

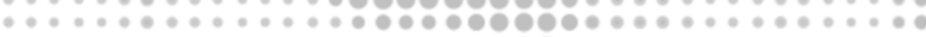

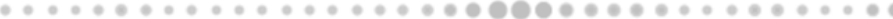

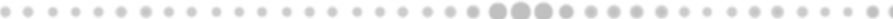

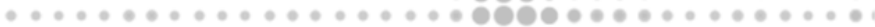

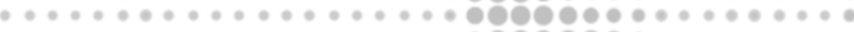

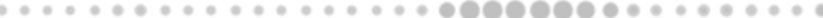

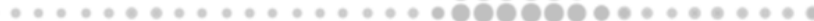

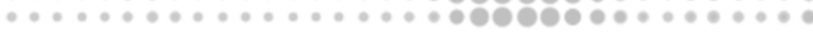

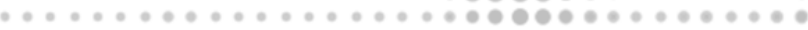

"

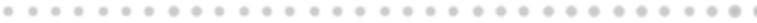

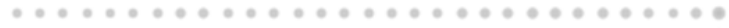

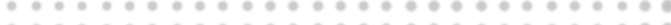

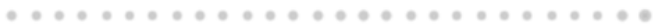

:

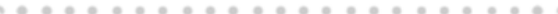

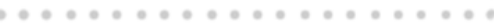

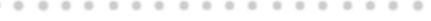

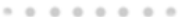





\section{THE NATURE OF HEARING}

Nature provides us with an ingenious organ to fulfill the sense of hearing. Human hearing occurs by retrieving sound from its surroundings (outer ear) and transferring, amplifying and delivering these sounds (middle ear) to the actual hearing organ named 'cochlea' (inner ear). Here hair cells translate the acoustic vibrations into neural pulse trains whilst conserving all necessary information to be transmitted to and interpreted by the human brain. During the course of human evolution the sense of hearing unfolded itself from a survival requirement to a valuable communication and interaction tool in human society today. As a consequence hearing loss is known to be one of the most disabling and challenging sensory dysfunctions with impact on the quality of human life (Hallberg et al, 2008; Kramer et al, 2002; Mulrow, 1990; Nachtegaal et al, 2012; Punch et al, 2019).

\section{UNILATERAL COCHLEAR IMPLANTATION}

Cochlear implantation (CI) makes it possible to adults and children with severe sensorineural hearing loss and limited benefit of conventional hearing aids (HA) to retrieve access to the auditory world. By inserting an electrode array in the inner ear, the impaired hair cells of the cochlea are functionally bypassed and direct multi-channel electrical stimulation of the auditory nerve fibres can provide information to the auditory system. The technology of cochlear implants (CI) has been applied successfully since the 1980s (House, 1976) and since the 1990s it is considered to be standard care for the profound to severely hearing impaired.

As in many other countries current reimbursement regulations in the Netherlands allow cochlear implantation to be performed only in one ear. Exceptions are made for supporting bilateral implantation in young children, in case of acute cochlear obliteration or in a small group of severely visually impaired subjects. The outcome of cochlear implantation is known to depend on many factors such as demographics, hearing history and etiology (Blamey et al, 2012; Kim et al, 2018; McRackan et al, 2019; Pisoni et al, 2017; le Roux et al, 2017). After an extensive rehabilitation period, unilateral implantation can in many cases enable postlingually deafened adults to understand speech again (Bierer et al, 2016; Van de Heyning et al, 2004; Krueger et al, 2008; Parkinson et al, 2002; Zwolan et al, 2001). However, a natural sound experience and performance of complex auditory tasks (e.g. speech understanding in difficult listening conditions and sound localization) remain a challenge since the signal coded by a CI only provides basic unilateral hearing in many of these patients (D'Alessandro et al, 2018; Dorman \& Gifford, 2017; Faulkner \& Pisoni, 2013; Runge et al, 2018; Wilson \& Dorman, 2008). 


\section{BIMODAL HEARING}

In view of the successful results of cochlear implantation in profoundly hearingimpaired people and through advances in signal processing, speech processors, implants and surgical techniques, the indications for a CI have expanded to include moderate to severe hearing losses (Gifford et al, 2010; Hughes et al, 2014; Huinck et al, 2019; Leigh et al, 2016) and asymmetric hearing (Firszt et al, 2018; Lepage et $\mathrm{al}, 2019)$. This means that in many cases unilaterally implanted patients still have aidable residual hearing in the non-implanted ear which may profit from acoustic amplification delivered by a conventional hearing aid. Contralateral residual hearing is mostly situated in the low frequency region (D'Alessandro et al, 2018; Dorman et al, 2015; Yehudai et al, 2012), while a cochlear implant by its insertion routing primarily stimulates the basal part of the cochlea which is sensitive for high frequency hearing. Taking advantage of combining two inputs in contralateral ears, electrical stimulation by a CI in one ear and acoustic amplification by a HA in the opposite ear, is known by the label of 'bimodal hearing'.

TABLE 1. Bimodal hearing: components and benefits.

\begin{tabular}{|cc}
\hline BIMODAL HEARING & CONTRALATERAL EAR \\
\hline IMPLANTEDEAR & Hearing aid (HA) \\
\hline $\begin{array}{c}\text { Cochlear implant (CI) } \\
\text { Direct electric stimulation of never fibres } \\
\text { Mainly high frequent information }\end{array}$ & $\begin{array}{c}\text { Acoustic amplification of residual hearing } \\
\text { Mainly low frequent information }\end{array}$ \\
\hline Bilateral input \\
Binaural cues \\
Complementary information
\end{tabular}

\section{BIMODAL PROFITS}

Bimodal benefit, defined as the benefit of listening with a $\mathrm{CI}$ and $\mathrm{HA}$ together compared to listening with the unilateral $\mathrm{CI}$ alone, is attributed to the combined effect of the use of two ears (bilateral input), the opportunity to centrally combine the input in both ears (binaural cues) and the access to complementary information (electric-acoustic, high and low frequencies). In a sense bimodal hearing has the merit of combining the best of both worlds, resulting in an outcome that can outperform each input in itself (Warren \& Dunbar, 2018).

Bimodal hearing has demonstrated to result in an improved ability to understand speech in difficult listening situations and to localize sounds (Ching et al, 2004; Ching et al, 2007; Gifford et al, 2007; Van Loon et al, 2017; Lotfi et al, 2019; Morera et al, 2012; Olson \& Shinn, 2008; Schafer et al, 2011). Other benefits across a range of hearing functions include improved listening for music and speech quality 
(Cheng et al, 2018; Ching et al, 2004; Crew et al, 2016; Cullington \& Zeng, 2011; Looi et al, 2007). It has also been noted that bimodal listeners report greater ease of listening, improved auditory spatial organization, more balanced sound and overall enhanced sound quality (Potts et al, 2009; Wallhäusser-Franke et al, 2018). Another advantage of contralateral stimulation by a hearing aid is that it may protect against auditory deprivation, the deterioration of auditory function by nonuse of the residual hearing (Polonenko et al, 2018; Silman et al, 1993).

\section{BIMODAL CHALLENGES}

In contrast to the theoretical benefits of bimodal aiding, there still remain a lot of unanswered questions and concerns within the field of bimodal hearing. A consensus statement states that all subjects with aidable residual hearing in the contralateral ear and good HA performance should be advised to try bimodal stimulation (Offeciers et al, 2005). No clear criteria exist however on the definition of 'aidable' residual hearing or 'good' HA performance (Scherf \& Arnold, 2014). As a consequence, and co-related to the changing CI-candidacy criteria over time, the number of patients preferring to retain a hearing aid after unilateral cochlear implantation diverges across studies, ranging from 10 to 60\% (Cowan \& ChinLenn, 2004; Fitzpatrick et al, 2009; Scherf \& Arnold, 2014; Syms et al, 2002; Tyler et al, 2002; Yamaguchi \& Goffi-Gomez, 2013).

Secondly, there is a large variability across bimodal users concerning the degree of objectified benefits (Ching et al, 2005; Hay-McCutcheon et al, 2018; Schafer et al, 2011). No clear correlations are found between the extent of bimodal benefit and the amount of residual hearing (Ching et al, 2007; Fitzpatrick et al, 2009; Hoppe et al, 2018). Moreover rather low correlations are found between objective benefits and self-reported outcomes (Noble et al, 2008b; Wallhäusser-Franke et al, 2018).

Thirdly, it is unclear if a derangement may occur between electric and acoustic stimulation across ears (Warren \& Dunbar, 2018). It has been described that some subjects can experience a degree of interference when combining bimodal devices (Fitzpatrick et al, 2009). This may be a consequence of the fact that electric hearing by a CI and acoustic hearing by a HA actually are two distinct worlds presented by two independent systems. This can introduce differences between both inputs (bilateral) and between both ears (binaural). Differences in signal processing and fitting procedures can give rise to a different percept of loudness, frequency representation, timing and sound quality, restraining the potential of bimodal benefits (Francart \& McDermott, 2013). Indeed no general agreement of best practice exists concerning how to optimally fit bimodal devices (Scherf \& Arnold, 2014; Siburt \& Holmes, 2015; Vroegop et al, 2018a). 


\section{OUTLINE OF THIS THESIS}

The goal of this thesis is to gain more insight into the field of bimodal hearing, specifically regarding the following research questions:

\section{WHO ARE CURRENT DAY BIMODAL USERS?}

Unilateral CI recipients can benefit from using a conventional hearing aid in the contralateral ear. It is however unclear which CI recipients are most likely to become bimodal users in the first place. This question is relevant when counseling $\mathrm{CI}$ candidates to provide them with the best fitted hearing solution. The first aim of this thesis is to investigate the preference of unilateral CI recipients whether to continue or discontinue the use of their contralateral HA after receiving a CI. Chapter 1 describes a retrospective cross-sectional database study among a recent CI population. The relation between the preference of retaining a contralateral HA and the following factors is investigated: demographic information, hearing history, residual hearing thresholds and the functional hearing status of each ear as well as the difference between both ears. In an attempt towards clinical guidelines, the secondary goal of this study is to determine threshold values for the most relevant factors in order to identify patient characteristics that best describe those unilateral CI recipients who are most likely to become bimodal users.

\section{WHAT ARE THE EXPERIENCED BENEFITS OF BIMODAL HEARING IN DALLY LIFE?}

Aside from objectified results, subjective patient related outcomes are very important to take into account when evaluating and improving health care (Farnik \& Pierzchała, 2012). Although many studies report bimodal benefit in laboratory situations, there still is a lack of studies exploring benefits reported by bimodal recipients themselves when wearing combined bimodal devices in daily listening situations. In Chapter 2 we send a set of daily-life questionnaires on bimodal HA use, hearing disability, hearing handicap and general quality of life to a cohort of unilateral CI-recipients. We question whether findings from previous literature (Fitzpatrick \& Leblanc, 2010; Noble et al, 2008a; Noble et al, 2008b) on the experiences of subjects who wear versus those who do not wear a contralateral hearing aid can be replicated. Moreover, the novel question in this study is to assess in which daily listening situations bimodal users themselves report benefit of their bimodal combination.

\section{HOW IS SPEECH PERCEPTION IMPROVED BY BIMODAL HEARING?}

Optimizing speech perception is the common incentive for hearing rehabilitation and consequently the clinically reported motivation of many subjects to continue 
the bimodal hearing combination after receiving a unilateral CI. We question how to capture and quantify the potential benefits of bimodal aiding regarding speech perception across the whole range of bimodal CI listeners. Traditionally speech perception is tested using intelligibility scores in quiet, while speech intelligibility in noise is often considered as a better measure since it would reflect real-life listening situations more appropriately. Measuring speech intelligibility in noise is however only feasible in subjects who already perform good in quiet. Yet, speech perception is more than intelligibility performance alone. It is a multidimensional concept in itself that entails amongst others also the ease and the naturalness of listening. In Chapter 3 we compile a test battery to evaluate the bimodal benefits on speech perception in a representative group of bimodally aided recipients. Speech intelligibility testing in quiet and in noise is supplemented with listening effort ratings and a sound quality questionnaire.

\section{CAN DIRECTIONAL MICROPHONES IMPROVE BIMODAL SPEECH PERCEPTION?}

Speech perception in demanding listening situations remains a challenge for many hearing impaired subjects and in particular CI recipients. Bimodal aiding is known to improve speech perception. Directional microphones are a proven preprocessing technique to enhance the desired signal in front while attenuating interfering sources around. This technique has nowadays entered the market of clinically available hearing aids as well as CIs. We question whether directional microphones and the bimodal combination of a CI and HA are complementary in improving speech intelligibly in noise. Chapter 4 describes the evaluation of a monaural beamformer in bimodal CI recipients. A repeated measures design is used to compare different directional configurations across CI and HA.

\section{WHAT FUTURE DIRECTIONS MIGHT IMPROVE THE BIMODAL EXPERIENCE?}

Nowadays cochlear implants are fitted with a one size fits all principle regarding the frequency representation across the electrode array. An insertion to the full extent of the cochlea is impossible and the exact location of the electrodes in the individual cochlea shows a large variability. A mismatch is therefore known to occur between the natural tonotopy at the cochlear electrode location and the frequency that CI processing presents at this electrode. It can be hypothesized that this mismatch not only has an influence on the learning process and the eventual outcome with a CI in itself but moreover plays a role when combining a CI with the natural residual hearing in the contralateral ear, as in the case of bimodal aiding. Chapter 5 presents a case-report of a single-sided deaf subject as a proof-ofprinciple study regarding an improved 3D imaging analysis technique to predict the pitch percept on CI electrode positions as a method towards a more natural CI frequency representation and a more optimal bimodal combination. 



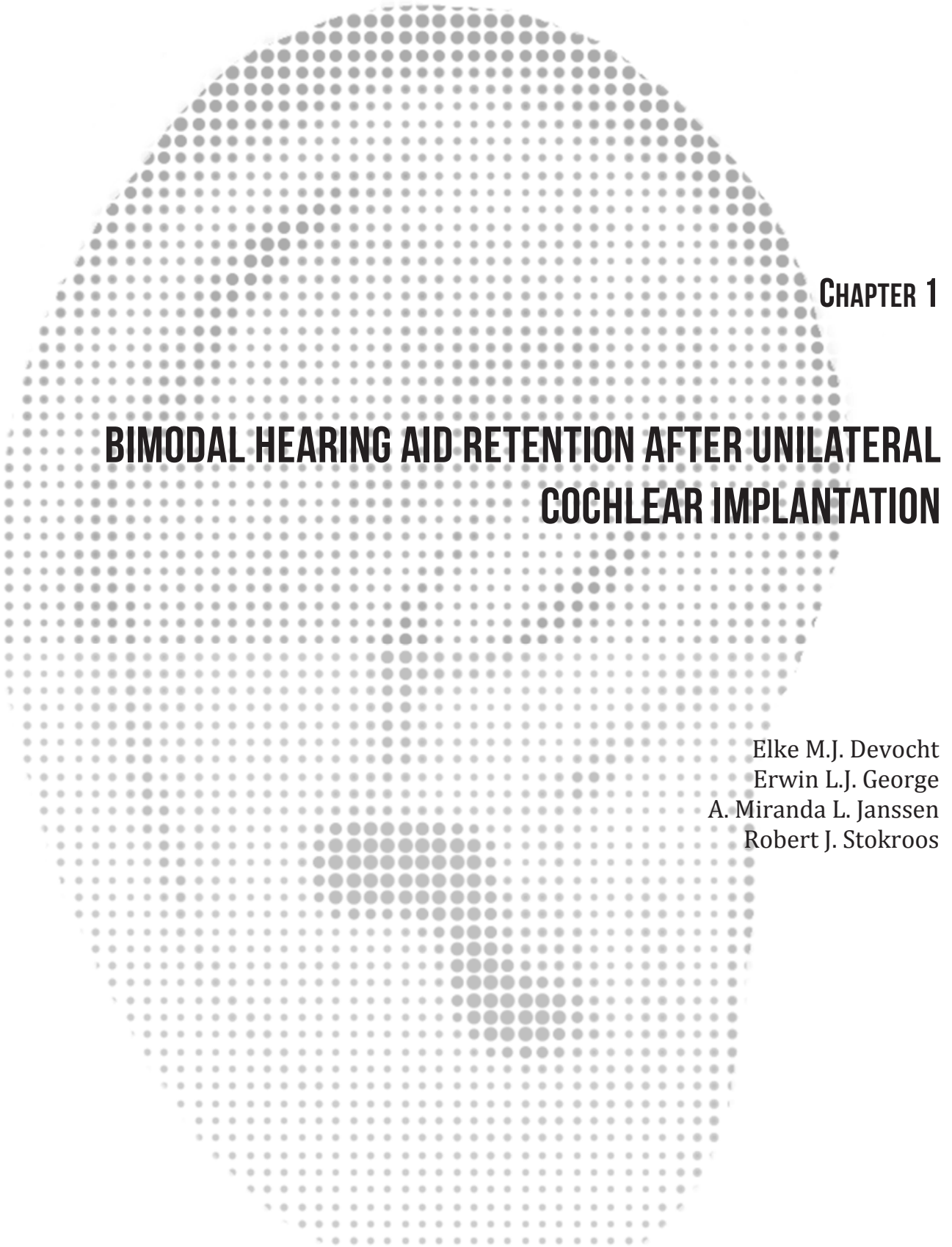

Audiology \& Neurotology, 2015;20:383-393 
The goal of this study was to investigate contralateral hearing aid (HA) use after unilateral cochlear implantation and to identify factors of influence on the occurrence of a unilateral cochlear implant (CI) recipient becoming a bimodal user. A retrospective cross-sectional chart review was carried out among 77 adult unilateral CI recipients 1 year after implantation. A bimodal HA retention rate of $64 \%$ was observed. Associations with demographics, hearing history, residual hearing and speech recognition ability were investigated. Better pure-tone thresholds and unaided speech scores in the non-implanted ear, as well as a smaller difference in speech recognition scores between both ears, were significantly associated with HA retention. A combined model of HA retention was proposed, and cutoff points were determined to identify those CI recipients who were most likely to become bimodal users. These results can provide input to clinical guidelines concerning bimodal CI candidacy. 


\section{INTRODUCTION}

With inclusion criteria broadening over the years (Gifford et al, 2010; Hughes et al, 2014), the number of cochlear implant (CI) candidates keeps increasing. Many still have useful residual hearing in the non-implanted ear and can therefore be fitted with a conventional hearing aid (HA). When electric hearing by means of a CI in 1 ear is supplemented with acoustic hearing by use of a conventional HA in the opposite ear, one speaks of bimodal hearing. This gives several advantages: the use of 2 ears (bilateral), the opportunity to centrally combine the input in both ears (binaural) and access to complementary information. It has been repeatedly demonstrated that bimodal hearing can provide benefit for speech recognition in noise, sound source localization, sound quality and music appreciation (Ching et al, 2007; El Fata et al, 2009; Olson \& Shinn, 2008; Schafer et al, 2011).

Many researchers have demonstrated benefit from the HA in bimodal patients. Yet survey studies show that how patients perform in laboratory tests is not always related to how they rate their abilities in everyday situations (Noble et al, 2008b). Thus, more research into stated patient preference regarding bimodal hearing is warranted. For instance, few studies have examined the number and type of patients who choose to use bimodal hearing in daily life (Cowan \& Chin-Lenn, 2004; Fitzpatrick et al, 2009; Yamaguchi \& Goffi-Gomez, 2013).

There is uncertainty about the rates of contralateral HA use after unilateral implantation. Most studies report bimodal rates of only 10-25\% (Fitzpatrick et al, 2009; Syms et al, 2002; Tyler et al, 2002; Yamaguchi \& Goffi-Gomez, 2013). This was countered by a demographic study among 71 adult CI recipients, showing that 75\% tried wearing an HA and 59\% continued using bimodal devices in daily life (Cowan \& Chin-Lenn, 2004). Then again, a recent survey study in CI centres around the world revealed that, within the total population of adult unilateral CI recipients, on average 32\% are bimodal users (Scherf \& Arnold, 2014).

Divergent bimodal rates could pertain to particular patient populations or point to differences in clinical practices (Siburt \& Holmes, 2015). There is no consensus on which patients are good candidates for the simultaneous use of a CI and a contralateral HA (Scherf \& Arnold, 2014), except the presence of usable residual hearing (Offeciers et al, 2005). The degree of residual hearing in CI patients however has progressively increased over the years, therefore lower bimodal rates may be related to stricter CI candidacy criteria in the past (Ching, 2005). A study by Fitzpatrick et al. (2009) showed a significant difference in time since implantation between the unilateral and bimodal patient groups. This study also considered age at implantation, duration of deafness and severity of hearing loss as 
possible contributing factors. They found a small but significant association between unaided hearing thresholds in the non-implanted ear and the likelihood of using a contralateral HA.

It is known that the prolonged lack of amplification has an effect on functional hearing performance with an HA (Silman et al, 1993). In that light, it is conceivable that the amount of HA experience before implantation could play a role in subsequently retaining the HA in the contralateral ear. From questionnaire results, it appears that a patient's satisfaction with CI results also influences the bimodal decision process (Fitzpatrick \& Leblanc, 2010). Van Hoesel (2012) showed that the bimodal benefit increased as the HA performance improved relative to that with the CI alone. A study by Yoon et al. (2014) recently suggested that the benefit of a bimodal fitting is facilitated when the performances of both modalities, electric and acoustic, are similar. Therefore, it is plausible that the outcome difference between both ears could influence bimodal HA retention as well.

\section{CuRRENT Study}

Studies investigating the added value of an HA in bimodal patients are numerous. Meanwhile, little research is carried out to assess which unilateral CI recipients are most likely to become bimodal users in the first place. Addressing this question is however very relevant in counselling unilateral CI recipients and providing them with a tailored fitting. The primary goal of the current study was therefore to further investigate the (dis)continuation of contralateral HA use after cochlear implantation and to identify factors contributing to bimodal HA retention.

A retrospective study with a cross-sectional design was carried out within a recent patient population, investigating a large set of factors related to bimodal HA use. Demographic information and HA history were factored in the study. Aside from residual hearing thresholds, it was assumed that also the functional hearing status of the contralateral ear, referring to speech recognition ability, played a role in bimodal HA retention. Furthermore, the outcome with CI and the difference in speech recognition ability between ears were hypothesized to be related to bimodal HA use as well.

The secondary aim of this study was to determine which threshold values best discriminate between HA retention and HA termination. Their determination could be a first step towards clinical guidelines to identify which unilateral CI patients are most likely to retain their HA after receiving a $\mathrm{CI}$ and hence become bimodal users. 


\section{Materials AND MethodS}

\section{ETHICS}

This study was conducted in accordance with national legislation, the medicalethical standards of the local institutional review board and the principles stated in the Declaration of Helsinki. Patients included in the study population declared no objection to the use of clinically obtained data for medical research.

\section{SUBJECTS}

The clinical charts of the adult patients of the CI team South-East Netherlands were reviewed. A potential bimodal candidate was defined as a unilateral CI recipient who reported to regularly (more than $50 \%$ of the time) use a conventional HA in the non-implanted ear prior to receiving the CI. All patients who had received a unilateral CI of the brand Advanced Bionics (Hires 90K CI system) in the period 2004-2013 and had come to the CI centre for yearly follow-up were selected $(n=86)$. Within this group, 77 subjects $(89.5 \%)$ were identified as potential bimodal candidates and were therefore included in the study population. In addition to demographic information (sex and age at implantation), details on hearing history were collected: duration of self-reported hearing loss in the nonimplanted ear, HA experience in the non-implanted ear and bilateral HA experience before implantation.

\section{Procedures}

For all patients in the study population, cross-sectional clinical data were reviewed 1 year after cochlear implantation. By then, the outcome situation is deemed to be stable (Luntz et al, 2005; Luntz et al, 2007). Of primary interest was the continuation of HA use in the contralateral ear, referred to as bimodal HA retention. Besides demographic information and details on hearing history, possible associations with the outcome were considered to lie in tone and speech audiometric measures. Results were collected unilaterally with the device in the other ear turned off. When testing the CI ear, the HA was kept in situ whereby the patient's own ear mold acted as a damping plug. For the non-implanted contralateral ear, aided results were never available for those patients who did not continue to use an HA. To ensure consistency, only unaided contralateral puretone thresholds and unaided contralateral speech recognition scores were included in the analysis. After all, both unaided and aided measures of speech recognition are known to be related to the same underlying speech recognition ability (Humes, 2002). All tests were performed in a sound-attenuated room under 
headphones in the unaided condition, and in free field at ear level at $1 \mathrm{~m}$ distance of a loudspeaker in the aided condition.

\section{BIMODAL HA RETENTION}

Bimodal HA retention was determined to be positive if clinical chart documentation stated that the patient reported to habitually use (more than $50 \%$ of the time) a contralateral HA in combination with the CI 1 year after implantation. Patients with positive HA retention were denoted as the bimodal group', while patients who had chosen to discard the HA were denoted as the 'unilateral' group. No strong recommendations were made by the rehabilitation team upon the continuation of contralateral HA use after implantation. HA use was mainly patient-driven and therefore represented stated patient preference regarding bimodal hearing.

During the first weeks after implantation, the primary focus of rehabilitation was the performance with CI. In clinical practice, no systematic bimodal fitting protocol was applied, not even over time, for a simple reason. Although adapting HA and/or CI fitting to optimize bimodal benefit is suggested in the literature (Francart \& McDermott, 2013), no generally accepted bimodal fitting method exists. In general, the clinical approach described in this study is characteristic of the current course of bimodal practice in CI centres around the world (Scherf \& Arnold, 2014).

\section{RESIDUAL HEARING}

Pure-tone audiometric thresholds were clinically collected at octave frequencies from 250 up to $8,000 \mathrm{~Hz}$. If no response was recorded at the maximum output of the audiometer, a value of $5 \mathrm{~dB}$ HL greater than the maximum tested level was noted. The pure-tone average (PTA) was calculated as the mean threshold at 500, 1,000 and 2,000 Hz. For all patients in the study population, the unaided PTA in the non-implanted contralateral ear was determined as a measure of residual hearing ('PTA contra UA'). The threshold at $250 \mathrm{~Hz}$ in the contralateral ear ('250 Hz contra UA') was considered as a separate factor. In CI candidates, thresholds at low frequencies commonly show the largest range of residual acoustic hearing. Furthermore, researchers previously demonstrated that mainly low-frequency information ( $<500 \mathrm{~Hz}$ ) contributes to bimodal benefit (Büchner et al, 2009; Illg et al, 2014; Mok et al, 2010; Sheffield \& Gifford, 2014; Zhang et al, 2010). Therefore, residual hearing at low frequencies could relate to bimodal HA retention as well. In the implanted ear, data on aided PTA with CI were gathered ('PTA CI') as a measure of auditory detection outcome with CI. 


\section{SPEECH RECOGNITION}

Speech audiometry was conducted in a quiet setting using a Dutch monosyllabic consonant-nucleus-consonant (CNC) test (Bosman \& Smoorenburg, 1995). The maximum score (\%) was determined over the levels up to $120 \mathrm{~dB}$ SPL for the nonimplanted ear in the unaided situation ('CNC contra UA') and over the levels 55, 65 and $75 \mathrm{~dB}$ SPL for the aided condition with $\mathrm{CI}$ ('CNC CI'). The difference in speech recognition ability between the two ears was also considered to possibly contribute to bimodal HA retention. This difference was defined as the maximum aided score with CI minus the maximum unaided contralateral score ('CNC CI CNC contra UA').

\section{Data ANalysis}

Demographic information and clinical test results 1 year after cochlear implantation, for both the bimodal and the unilateral group, were described by means and standard deviations.

Univariable logistic regression analyses were performed to investigate the association between each of the patient characteristics and the status of bimodal HA retention 1 year after cochlear implantation. The univariable odds ratios are presented along with the $95 \%$ statistical confidence intervals and the corresponding $p$ value. Statistical significance was assumed at $p<0.05$. The Hosmer and Lemeshow test was performed to inspect the goodness of fit of the logistic model. Since the null hypothesis of this test is a good model fit, a nonsignificant $\mathrm{p}$ value implies that the model describes the data well.

For each significant contributing factor a receiver-operating characteristic curve (ROC) was studied, and the area under the curve (AUC) was used to assess the ability of the factor to discriminate between positive HA retention and discontinued HA use. An ROC curve plots the true-positive rate (sensitivity) against the false-positive rate (1 - specificity) at any given value of the factor under investigation (Metz, 1978; Zweig \& Campbell, 1993). The AUC is equal to the probability that an observer will correctly identify the positive case when presented with a random positive-negative case pair (Hanley \& McNeil, 1982). Hereby an AUC of 0.5 reflects no discrimination above chance, whereas an AUC of 1.0 indicates perfect discrimination. Generally, AUC values of $\geq 0.90$ are considered excellent, 0.80-0.89 good, 0.70-0.79 fair and $<0.70$ poor.

For factors with an AUC significantly different from 0.5, the most optimal cut-off point with corresponding specificity and sensitivity was determined using the maximum value of the Youden index (Youden, 1950). The optimal cut-off point is the discrimination threshold that reflects the best performance of the factor as a 
classifier of the outcome between positive and negative HA retention, considering the best trade-off between test sensitivity and specificity.

A multivariable logistic regression model was constructed by methods of backward stepwise selection with a drop-out criterion of $\mathrm{p}>0.10$. Those variables that were significantly associated with HA retention in the univariable analysis were considered candidate factors for the multivariable analysis. Also for the multivariable model, a Hosmer and Lemeshow test and ROC curve analysis were performed, the AUC was assessed, and the optimal cut-off point, as a trade-off between the different remaining factors, was determined.

All statistical analyses were performed using IBM SPSS Statistics version 22.0.0.1.

\section{RESULTS}

\section{Patient Characteristics}

One year after implantation, 49 patients in the study population continued to habitually use an HA in the contralateral ear, while the other 28 patients terminated HA use. This amounts to an HA retention rate of $63.6 \%$.

Demographic information, details on hearing history and audiological test results for the subgroups of bimodal and unilateral users are presented in table 1 . For some patients, details on duration of hearing loss and HA experience could not be derived retrospectively from clinical charts. Therefore, the obtained sample size is listed for those variables where it does not equal the total study population.

TABLE 1. Characteristics of Study Population and Results of Univariable Logistic Regression Analysis

\begin{tabular}{lcc|cccc} 
Variable (unit) & Bimoda & Unilateral & \multicolumn{3}{c}{ Univariable logistic } \\
& $N=49$ & $N=28$ & OR & $95 \%$ CI $_{\text {stat }}$ & $p$ & 0.079 \\
Sex (No. male/female) & $23 / 26$ & $19 / 9$ & 0.4 & {$[0.16-1.12]$} & 0.9 \\
Age at implantation (yrs) & 59.9 & $61.2(17.1)$ & 0.9 & {$[0.96-1.03]$} & 0.703 \\
Duration hearing loss contra (yrs) (n=72) & 31.3 & $34.4(14.9)$ & 0.9 & {$[0.96-1.02]$} & 0.434 \\
HA experience contra (yrs) (n=60) & 26.9 & $28.2(13.3)$ & 0.9 & {$[0.96-1.03]$} & 0.743 \\
HA experience bil (yrs) (n=43) & 20.2 & $29.0(9.9)$ & 0.9 & {$[0.91-1.00]$} & 0.057 \\
PTA contra UA (dB HL) & 92.3 & $102.1(14.0)$ & 0.9 & {$[0.91-0.98]$} & $0.005^{* *}$ \\
250 Hz contra UA (dB HL) & 76.7 & $88.4(21.9)$ & 0.9 & {$[0.96-1.00]$} & $0.045^{*}$ \\
PTA CI (dB HL) & 38.5 & $34.6(9.3)$ & 1.0 & {$[0.99-1.10]$} & 0.099 \\
CNC contra UA (\%) & 38.2 & $16.3(17.9)$ & 1.0 & {$[1.02-1.07]$} & $<0.001^{*}$ \\
CNC CI (\%) & 52.7 & $59.0(28.3)$ & 0.9 & {$[0.97-1.01]$} & 0.318 \\
CNC CI - CNC contra UA (\%) & 14.5 & $42.7(26.9)$ & 0.9 & {$[0.95-0.99]$} & $0.001^{* *}$
\end{tabular}

Patient characteristics at time of implantation and audiological test results one year after implantation: mean (standard deviation) and results of univariable logistic regression analysis for the outcome of $\mathrm{HA}$ retention $(* \mathrm{p}<0.05$ ** $\mathrm{p}<0.01$ ). OR, odds ratio; CIstat, 95\% confidence interval (statistics); CI, cochlear implant; HA, hearing aid; contra, non-implanted ear; bil, bilateral; yrs, years; UA, unaided measurement; PTA, pure-tone average at 500 - 1000 $2000 \mathrm{~Hz}$; CNC, Consonant Nucleus Consonant speech testing in quiet. 
A visual representation of the hearing loss in the non-implanted ear for both groups is displayed in figure 1 . The patients who discarded their HA $(n=28)$ had an average CNC score with the CI of 59\% (SD 28.3\%), while those patients who did retain their HA $(n=49)$ had average CNC scores of $52.7 \%$ (SD 25.7\%), 41.1\% (SD 23.6\%) and 66.1\% (SD 20.8\%) aided with CI alone, HA alone and CI and HA together, respectively.

FIGURE 1. Residual hearing thresholds in the non-implanted ear.

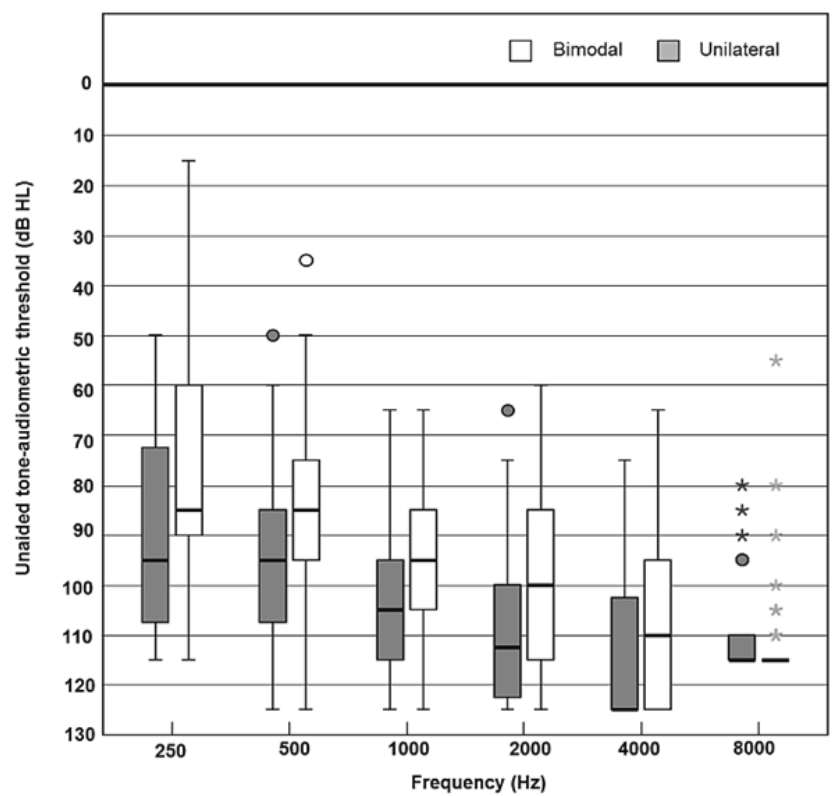

For the groups of unilateral and bimodal users 1 year after cochlear implantation. Box plots represent the distribution per frequency (median and interquartile range), with whiskers denoting minimum and maximum values within 1.5 times the interquartile range, circles denoting outliers and asterisks denoting extremes.

\section{UNIVARIABLE LOGISTIC REGRESSION}

Results of the univariable logistic regression analyses for each factor related to bimodal HA retention are included in table 1 . Age at implantation, duration of contralateral hearing loss, experience of HA use in the contralateral ear, bilateral HA experience and PTA and CNC scores with CI did not show a significant relationship with continuation of HA use in the non-implanted ear. Sex had a notable small odds ratio $(0.42)$ in association with HA retention but fell short of significance $(p=0.079)$. However, HA retention was shown to be highly significantly related to the general amount of residual hearing in the contralateral ear (PTA contra UA; $p=0.005$ ), the unaided speech scores in the contralateral ear (CNC contra UA; $p<0.001$ ) and the difference in speech recognition ability between both ears (CNC CI - CNC contra UA; $p=0.001$ ). The hearing threshold at 
$250 \mathrm{~Hz}$ ( $250 \mathrm{~Hz}$ contra UA) also showed an association with HA retention, although in comparison to the other factors it was found to be only just significant $(p=0.045)$. Hosmer and Lemeshow tests were not significant $(p>0.05)$, indicating a good fit for each of the univariable models.

Since HA use was defined as positive if an HA is retained after implantation, a significant odds ratio $>1$ means that $\mathrm{HA}$ retention was associated with higher values of the variable under investigation. On the other hand, an odds ratio $<1$ stands for an association between HA retention and lower values of the tested variable. As shown in table 1 , resulting odds ratios revealed that the probability of bimodal HA retention increased with lower (i.e. better) residual hearing thresholds (averaged and at $250 \mathrm{~Hz}$ ) and higher speech recognition scores in the contralateral ear. HA continuation was also related to a smaller difference in speech scores, referring to a functional outcome whereby the $\mathrm{CI}$ is less dominant over the HA ear. Figure $2 \mathrm{a}-\mathrm{d}$ illustrates the results for each of the four factors found to be significantly related to bimodal HA retention. Histograms represent the observed distribution of the factor under investigation for the bimodal group (open bars at the top) and for the unilateral group (shaded bars at the bottom). The fitted line, accompanied by its $95 \%$ confidence range, demonstrates the probability of bimodal HA retention predicted by the logistic model of the respective factors.

\section{UNIVARIABLEROC ANALYSIS}

Table 2 summarizes the results of the univariable ROC analysis. With significant AUC values of $0.72-0.76$, the factors PTA contra UA, CNC contra UA and CNC CI CNC contra UA exhibited fair accuracy in discriminating between positive and negative HA retention. For the factor $250 \mathrm{~Hz}$ contra UA however, discrimination accuracy was not significantly better than chance in defining HA retention ( $p=0.053$ ). This is related to the fact that the contralateral threshold at $250 \mathrm{~Hz}$ was only just significant in the earlier univariable logistic regression analysis.

For each of the other three significant factors, a cut-off point was determined, as displayed in figure $2 \mathrm{a}, \mathrm{c}$ and d. Evaluation of the cut-off points demonstrated that a PTA in the contralateral ear of less than $100.8 \mathrm{~dB} \mathrm{HL}$, an unaided speech recognition score in the non-implanted ear of more than $36 \%$ and a speech recognition difference of less than $34.5 \%$ in favour of the $\mathrm{CI}$ were associated with continued HA use after implantation. 
FIGURE 2. Probability of bimodal hearing aid (HA) retention one year after cochlear implantation.

A

B

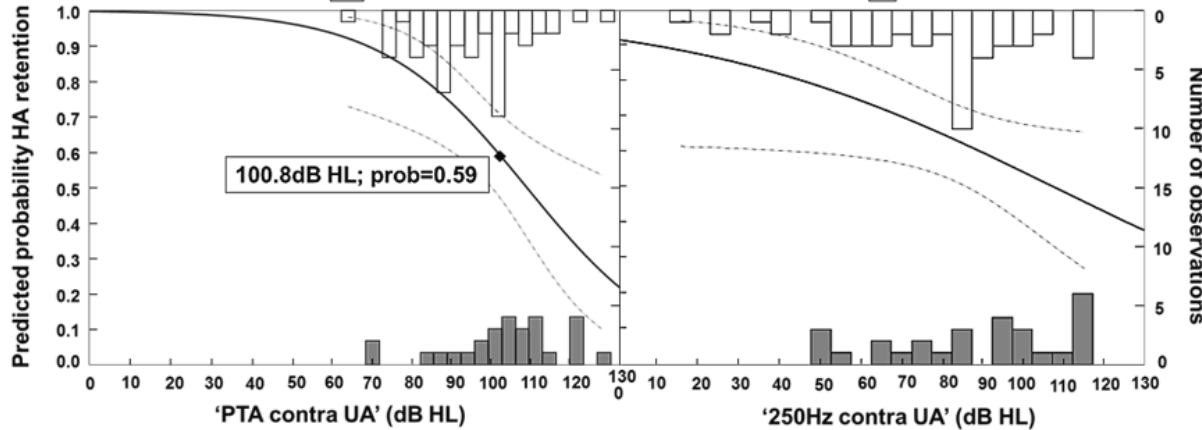

C

D

In

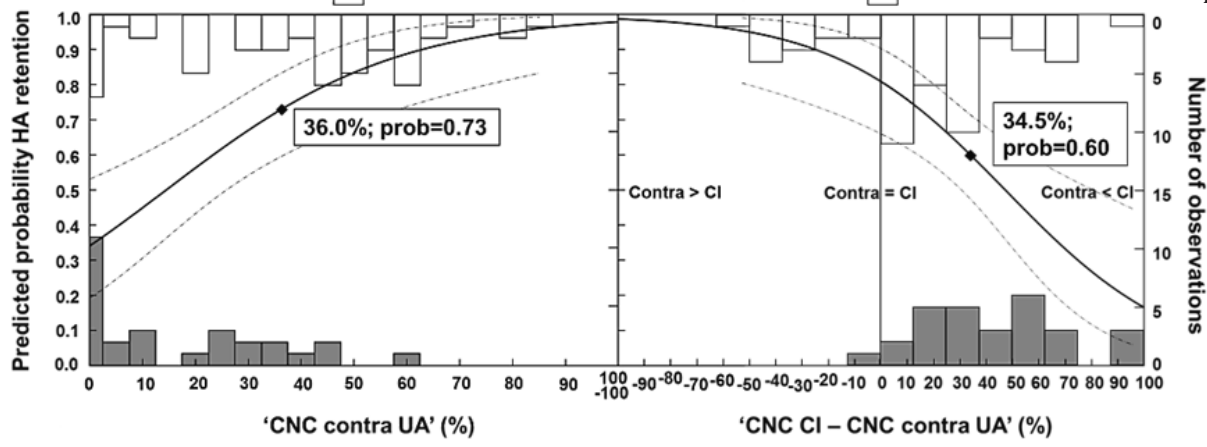

Observed Bimodal 대] Observed Unilateral _— Probability predicted by model _..-.- 95\% Confidence Interval

- Cut-off point ROC analysis

relation to the unaided pure-tone average $(500,1000,2000 \mathrm{~Hz})$ in the non-implanted contralateral ear ('PTA contra UA') (A); the unaided pure-tone threshold at $250 \mathrm{~Hz}$ in the non-implanted contralateral ear ('250 Hz contra UA') (B); the maximum unaided CNC score in the non-implanted contralateral ear ('CNC contra UA') (C); and the difference in maximum speech-recognition scores between the cochlear implanted ear and the unaided contralateral ear ('CNC CI CNC contra UA') (D).

TABLE 2. Results of ROC Analyses with Cut-Off Points.

\begin{tabular}{|c|c|c|c|c|c|c|}
\hline \multirow{2}{*}{ Model } & \multicolumn{2}{|r|}{ ROC } & \multicolumn{4}{|c|}{ Cut-off point } \\
\hline & AUC & $p$ & Sens & Spec & Pred Prob & Value \\
\hline \multicolumn{7}{|l|}{ Univariable } \\
\hline PTA contra UA & 0.72 & $0.002^{* *}$ & 0.78 & 0.61 & 0.59 & $100.8 \mathrm{~dB}$ \\
\hline $250 \mathrm{~Hz}$ contra UA & 0.63 & 0.053 & & & & \\
\hline CNC contra UA & 0.76 & $<0.001^{* *}$ & 0.59 & 0.86 & 0.73 & $36.00 \%$ \\
\hline CNC CI - CNC contra UA & 0.74 & $0.001^{* *}$ & 0.80 & 0.61 & 0.60 & $34.50 \%$ \\
\hline \multicolumn{7}{|l|}{ Multivariable } \\
\hline PTA contra UA; CNC CI - CNC contra UA & 0.78 & $<0.001^{* *}$ & 0.65 & 0.89 & 0.71 & \\
\hline
\end{tabular}

Results of ROC analysis $\left(* p<0.05,{ }^{* *} p<0.01\right)$ for significant univariable factors and the combined multivariable model. For those factors with a significant AUC, cut-off points are determined based on maximum Youden Index. ROC, Receiver Operating Characteristic curve; AUC, Area Under the ROC Curve; Sens, sensitivity; Spec, specificity; Pred Prob, predicted probability; $\mathrm{CI}$, cochlear implant; HA, hearing aid; contra, non-implanted ear; UA, unaided measurement; PTA, pure-tone average at $500-1000-2000 \mathrm{~Hz}$; CNC, Consonant Nucleus Consonant speech testing in quiet. 


\section{MuLTIVARIABLE LOGISTIC REGRESSION}

To investigate the relative combined contribution of the variables PTA contra UA, $250 \mathrm{~Hz}$ contra UA, CNC contra UA and CNC CI - CNC contra UA to bimodal HA retention, backward stepwise selection was applied to construct a multivariable logistic regression model:

(1) $\mathrm{P}(\mathrm{HA}$ retention $)=1 /(1+\mathrm{EXP}-[0.03(\mathrm{CNC}$ contra UA $)$

$$
-0.02(\mathrm{CNC} \text { CI - CNC contra UA) + 0.21]) }
$$

Hereby EXP indicates the natural exponential function, and P stands for the predicted probability of HA retention from the regression model. This model (equation 1) included both speech recognition in the contralateral ear (odds ratio 1.03, confidence interval $1.00-1.06, p=0.025$ ) and the difference between both ears (odds ratio 0.98, confidence interval $0.96-1.00, p=0.096$ ) as significantly contributing variables. However, the factors representing the mean unaided puretone audiometric threshold and the threshold at $250 \mathrm{~Hz}$ did not improve the predictive power. They were therefore not selected for inclusion in the combined regression model $(p=0.94$ and $p=0.82$, respectively). A Hosmer and Lemeshow test on the final model did not yield significance $(p>0.05)$, indicating a good fitted model. The fitted multivariable logistic model is illustrated in figure 3 . The greyscale rep resents the modelled probability of contralateral HA retention, with brightness indicating a higher predicted probability of bimodal HA continuation. The circles, which display the original data of the study population, are filled for subjects who discontinued HA use (unilateral group) and open for subjects who continued HA use (bimodal group).

\section{MULTIVARIABLE ROC ANALYSIS}

The result of ROC curve analysis for the multivariable model is shown in table 2 . The model discriminated with significant and fairly good accuracy (AUC 0.78) between positive and negative HA retention. The cut-off point function for HA retention based on the combination of outcome measures CNC contra UA and CNC $\mathrm{CI}$ is associated with a predicted probability of 0.71 and is illustrated by the fitted line in figure 3 . Within the range limits of the CNC outcome measures $(0-100 \%)$, this cut-off point function demonstrated that a maximum CNC word score $<13.8 \%$ in the unaided contralateral ear was associated with discontinued HA use after implantation. In contrast, a maximum CNC score $>49 \%$ in the unaided HA ear was associated with continued HA use. For an unaided CNC score in the non-implanted ear between 13.8 and $49 \%$, the CNC outcome with CI played a complementary role in the association with bimodal HA retention. 
FIGURE 3. Probability of bimodal hearing aid (HA) retention one year after cochlear implantation.

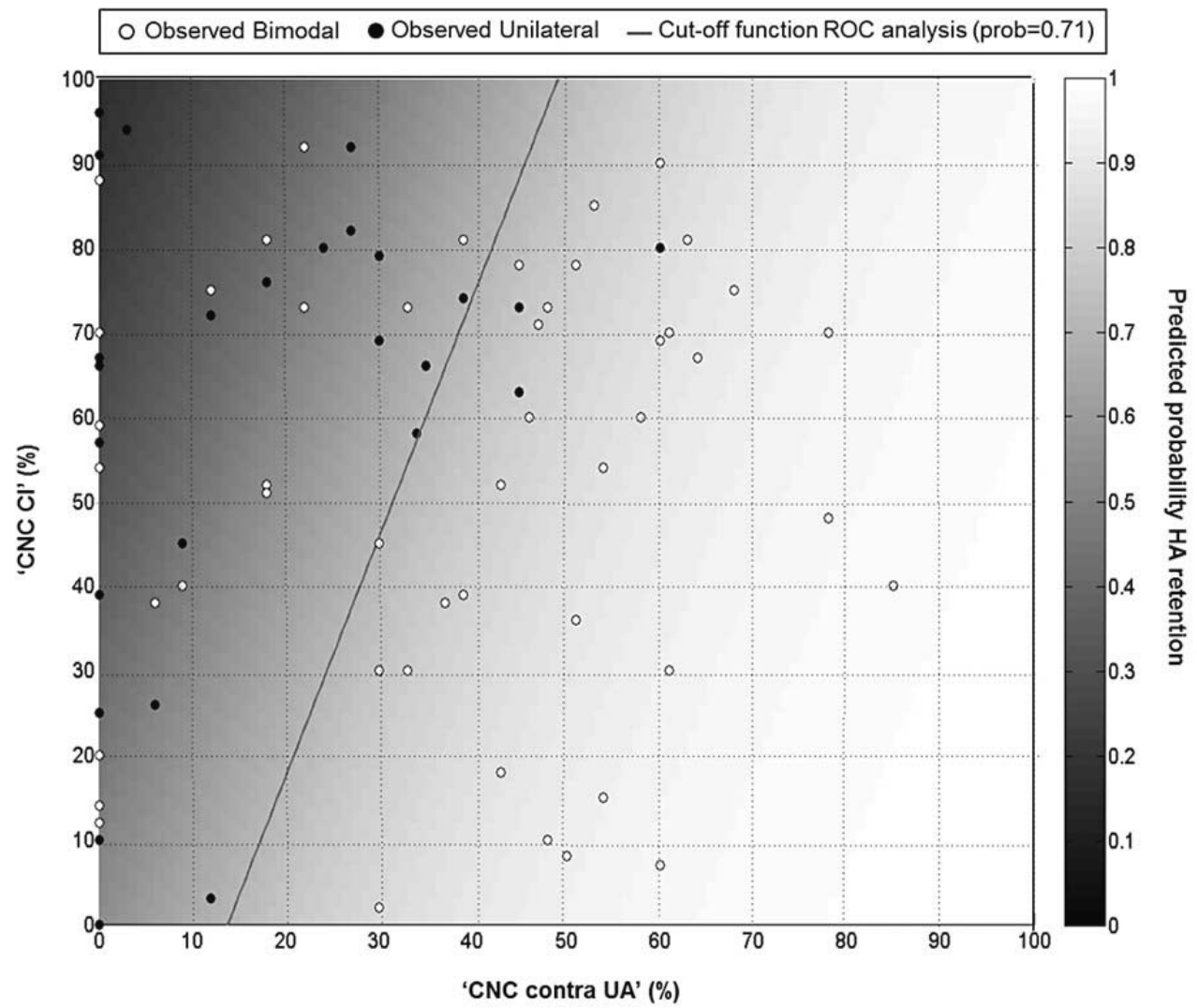

In relation to the maximum speech-recognition score in the cochlear implanted ear (' $\left.\mathrm{CNC} \mathrm{CI}^{\prime}\right)$ and the unaided contralateral ear ('CNC contra UA'). 


\section{DISCUSSION}

\section{BIMODAL HARETENTION}

The aim of this study was to investigate the continuation of contralateral HA use after unilateral cochlear implantation. For patients who wore a contralateral HA preoperatively, an $\mathrm{HA}$ continuation rate of $64 \%$ after 1 year of $\mathrm{CI}$ experience was observed. This bimodal rate is rather higher than the range of bimodal use (10 up to 59\%) retrieved from previous demographic studies (Cowan \& Chin-Lenn, 2004; Syms et al, 2002; Tyler et al, 2002). Previous studies never noted whether an HA had been used prior to implantation and therefore most likely looked into the total unilateral population. If the total unilateral population is considered in this study, still a rather high bimodal usage rate of 57\% (49 out of 86 subjects) was found. Unlike some of those previous studies, the present study placed no predefined restrictions on residual hearing for bimodal eligibility, yet a higher rate of HA retention was found. The higher rate is most likely an effect of inclusion criteria becoming less strict over the years. All previous studies cover patients implanted before 2004, while this study population contained more recent CI recipients (2004-2013). Its composition accounts for the difference in mean contralateral residual hearing thresholds (PTA) between this recent study population (95.9 dB HL) and that of a previous study (108.1 dB HL) by Fitzpatrick et al. (2009).

\section{CONTRIBUTNGG FACTORS}

Residual Hearing

The degree of residual contralateral hearing turned out to be significantly associated with HA retention. This is consistent with previous research (Yamaguchi \& Goffi-Gomez, 2013), just as the finding that average contralateral residual hearing (PTA) was a contributing factor, while the threshold specified at low frequencies did not seem to add any additional information (Fitzpatrick et al, 2009). Some earlier studies showed that low-frequency information contributes to bimodal benefit for speech recognition in noise, prosody and music perception since it is not well transmitted by the CI (Büchner et al, 2009; Illg et al, 2014; Mok et al, 2010). However, such complex auditory tasks were not addressed in the current study, which looked into the reasons for patients to become bimodal or unilateral users in the first place. Here results suggest usable residual hearing over a wide frequency range as reason for patients to retain their HA. This result can be related to other findings from the bimodal literature that state that a contralateral HA should be fitted to provide acoustic information over the whole range of 
frequencies with usable residual hearing, and not only at low frequencies (Neuman \& Svirsky, 2013; Sheffield \& Gifford, 2014).

\section{SPEECH RECOGNITION}

Speech recognition ability in the non-implanted ear also appeared to be related to HA retention after implantation. In fact, the multivariable model demonstrated that mainly speech recognition, assessed by the maximum CNC word recognition score in the non-implanted ear and the difference between both ears, was related to bimodal HA use. Tone audiometric hearing level did not add any additional information to the combined model. Although auditory detection is a prerequisite for functional hearing, these findings point to speech recognition as a more important factor contributing to bimodal HA retention in daily life.

Besides threshold information, it has been suggested that measures of suprathreshold information are also important for speech recognition (George et al, 2006; Glasberg \& Moore, 1989; Plomp, 1978; Stephens, 1976). Hearingimpaired subjects with comparable hearing sensitivity (i.e. pure-tone thresholds) would not necessarily achieve the same level of speech recognition (Skinner, 1982). The results of the current study highlight the importance of suprathreshold factors for the bimodal research field. Suprathreshold hearing incorporates functions such as frequency resolution, temporal resolution, temporal fine structure coding and loudness perception, but also linguistic skills and cognition (Bernstein \& Mehraei, 2013; Grant \& Walden, 2013; Kramer et al, 2009). As Zhang et al. (2013) recently suggested in a study on bimodal benefit, acoustic spectral modulation detection thresholds, as a measure of spectral resolution, may be stronger predictors of the degree of benefit than basic audiometric measurements.

Although in this study speech recognition proved to be the main factor associated with bimodal HA retention, it could not explain continued bimodal use for all patients. Figures $2 \mathrm{c}$ and 3 show that some subjects did continue to wear a contralateral HA, despite having little or no measurable speech recognition ability in the non-implanted ear. For those patients, other qualities of the acoustic ear may be important for retaining the HA, depending on the demands of their environment. For example, Kong et al. (2005) described 5 patients who, even though no speech recognition could be achieved with the acoustic ear alone, gained benefit from bimodal stimulation when listening to speech in background noise. This benefit was attributed to the ability of the acoustic ear to analyse the temporal fine structure of the acoustic scene. 


\section{DIFFERENCE BETWEEN EARS}

It has previously been suggested (Ching et al, 2004; Noble et al, 2008b), and demonstrated in a survey study (Fitzpatrick \& Leblanc, 2010), that CI outcome plays an important role in the bimodal decision-making process. When results with the $\mathrm{CI}$ are unsatisfying, a contralateral HA may provide adequate means to improve performance in daily listening situations. On the other hand, when $\mathrm{CI}$ results are already satisfying, an $\mathrm{HA}$ might be experienced as interfering with the outperformance of the CI.

Data from the current study suggest that it is not the CI outcome by itself but the difference between functional speech performance of both ears that significantly affects HA retention. It was seen that a between-ear difference score less in favour of the $\mathrm{CI}$ was related to HA continuation after implantation. If the HA outperforms the $\mathrm{CI}$, in case of a disappointing $\mathrm{CI}$ outcome, it seems evident that a patient will decide to continue HA use. However, the distribution of difference scores among bimodal users ( fig. $2 \mathrm{~d}$ ) reveals that most bimodal users had a comparable speech recognition ability in both ears (difference between 0 and 30\%). Apparently, functional across-ear comparability is connected to bimodal use. This observation is related to a recent study by (Yoon et al, 2015) on the benefit provided by a bimodal fitting. They demonstrated a significant correlation between bimodal benefit and the difference in performance between ears for speech recognition, with a smaller difference leading to greater bimodal benefit.

Finally, the importance of the difference between ears found in this study suggests that emerging patient preference takes the contralateral score as a reference point for performance. The postoperative $\mathrm{CI}$ outcome may then be weighed against that score when making the decision whether to opt for bimodal hearing.

\section{GENDER}

Sex approached but did not reach significance as a factor in the relationship with HA retention in the current study. Despite limited power, a trend towards male subjects having a lower rate of bimodal HA retention could be observed ( table 1 ). The underlying reasons for the found trend are unclear. A gender difference might be intermediated by the degree of hearing loss or related to attitude factors such as appearance. Even in the general field of HAs, there has been very little research to date that examines the differences between men and women in the use of HAs (McCormack \& Fortnum, 2013). In a study by Staehelin et al. (2011), women also reported a higher prevalence of daily and regular use of HAs. 


\section{DESIGN LIMITATIONS}

An even better representation of functional hearing would have been based on the aided speech reception scores with an HA. Indeed, it is reasonable to assume that HA fitting could also have played a role in the observed bimodal HA retention rate since insufficient gain could restrain the perceived bimodal benefit (Yehudai et al, 2013). However, the current retrospective study design of a cross-sectional review 1 year after implantation implied that postoperative aided data in the contralateral ear were not available for unilateral patients who discarded their HA. Furthermore, also pure-tone thresholds below $250 \mathrm{~Hz}$ were unavailable, since these are not routinely measured in clinical practice. A limitation of the current study therefore lies in its retrospective cross-sectional design in a clinical population. Moreover, since the outcome situation and the possible contributing factors were all collected at the same point in time (1 year after implantation), a predictive model in the strict sense of the word was not possible. Nevertheless, this design was chosen because it enabled exclusion of progressive hearing loss as a confounding factor and inclusion of the outcome with a CI. Recent research has demonstrated stability of contralateral residual hearing up to 2,000 $\mathrm{Hz}$ over more than 6 years after implantation (Yehudai et al, 2012). This means that hearing status 1 year after implantation can be considered a good estimate of preoperative hearing abilities.

\section{EvaluATION OF StUdY AIMS}

The primary aim of the current study was to investigate demographic and auditory factors related to bimodal HA retention. However, many non-auditory factors are known to impact HA satisfaction, such as aesthetics, stigma, habitude, cost and comfort (Wong, 2003). These latter factors contribute to the stated patient preference and may account for some of the unaccounted predictive power of the current model. Incorporation of more factors would

require a larger cohort and self-reported experiences with bimodal fitting. To this end, and to investigate follow-up use in the longer term, further research is warranted.

The secondary aim was to determine discrimination values to identify which unilateral CI patients are most likely to become bimodal users. Results are based on standard data from CI clinical practice. Therefore, these statistical outcomes can provide input for clinical guidelines on bimodal CI candidacy. Besides underpinning decision-making on which ear to implant, the outcomes can help distinguish between potential bimodal candidates and candidates for bilateral implantation. 
For residual hearing (PTA), a cut-off point of $100 \mathrm{~dB}$ HL was obtained. This lies within the range of criteria (from < 90 to $110 \mathrm{~dB}$ HL) applied in bimodal research to define usable residual hearing (Fitzpatrick et al, 2009; Neuman \& Svirsky, 2013; Park et al, 2012). For speech recognition ability in the contralateral ear, a cut-off point of 36\% was found, or a point somewhere between 14 and 49\% when looking at the model combined with the outcome of the CI. These results align with previous findings: subjects with pre-operative contralateral HA word scores of roughly $\geq 20 \%$ tend to have greater potential to derive benefit from bimodal stimulation than subjects scoring $<20 \%$ (Morera et al, 2005).

It may be inferred from these cut-off points that, with a CNC score of $>49 \%$ bimodal stimulation stands a good chance, that a score of $<14 \%$ warrants considering bilateral implantation, and that a score somewhere in between calls for considering the degree of achieved bimodal benefit before deciding upon bilateral implantation. It was also found that patients with more balanced speech recognition abilities (less than 35\% difference between the CI and non-implanted ear) have more chances of becoming a bimodal user. This result is in line with the findings of Yoon et al. (2015), who suggested that a speech performance difference of more than $30 \%$ would potentially cause bimodal interference.

Determining cut-off points in the univariable and multivariable ROC analysis is only the first step towards formulating clinical guidelines. These cut-off points have to be tested for validity in other patient populations and in a prospective manner before they could be relied upon as rules in everyday clinical practice.

\section{CONCLUSIONS}

This study investigated contralateral HA use after unilateral implantation. A retrospective cross-sectional chart review demonstrated a $64 \%$ rate of bimodal HA retention 1 year after implantation. Residual hearing and mainly speech recognition ability were identified as factors related to the occurrence of a unilateral CI recipient becoming a bimodal user 1 year after implantation. Patients who had more residual speech recognition ability in the non-implanted ear and featured a less dominant outcome of the CI over the HA ear were more likely to continue using a contralateral HA 1 year after receiving a unilateral CI. Although the criteria for bimodal candidacy are still unresolved, this study proposed discrimination values to identify which unilateral CI patients are most likely to turn into bimodal users. 


\section{DISCLOSURE OF INTEREST}

We acknowledge a research grant from Advanced Bionics Inc., which financially supported the work of the first author (E.D.) in this investigator-initiated study. The third author (M.J.) provided statistical support, made possible by a grant from the Dutch Heinsius-Houbolt Foundation. The authors declare no other conflicts of interest. 



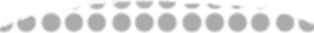

-

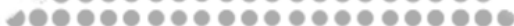

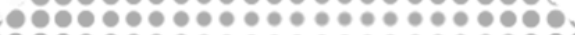

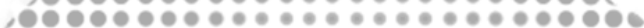

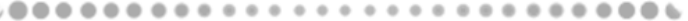

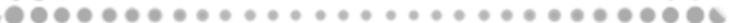

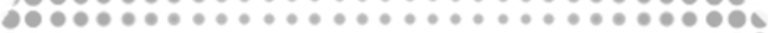



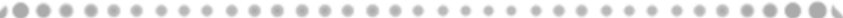

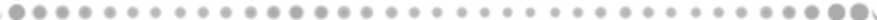

เ

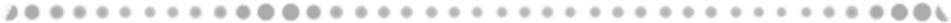

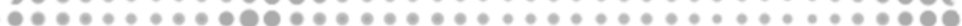

,

(5)

-

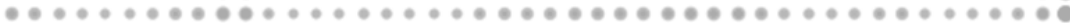

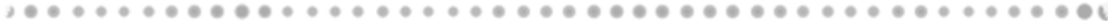

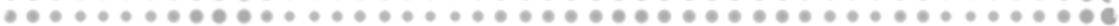

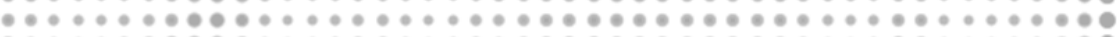

,

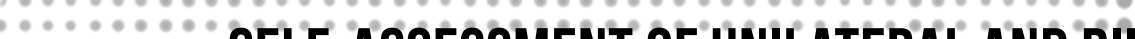

\section{SELF-ASSESSMENT OF UNIEATERAL AND BIMODAL COCHLEAR IMPLANT EXPERIENCES IN DAILY LIFE}
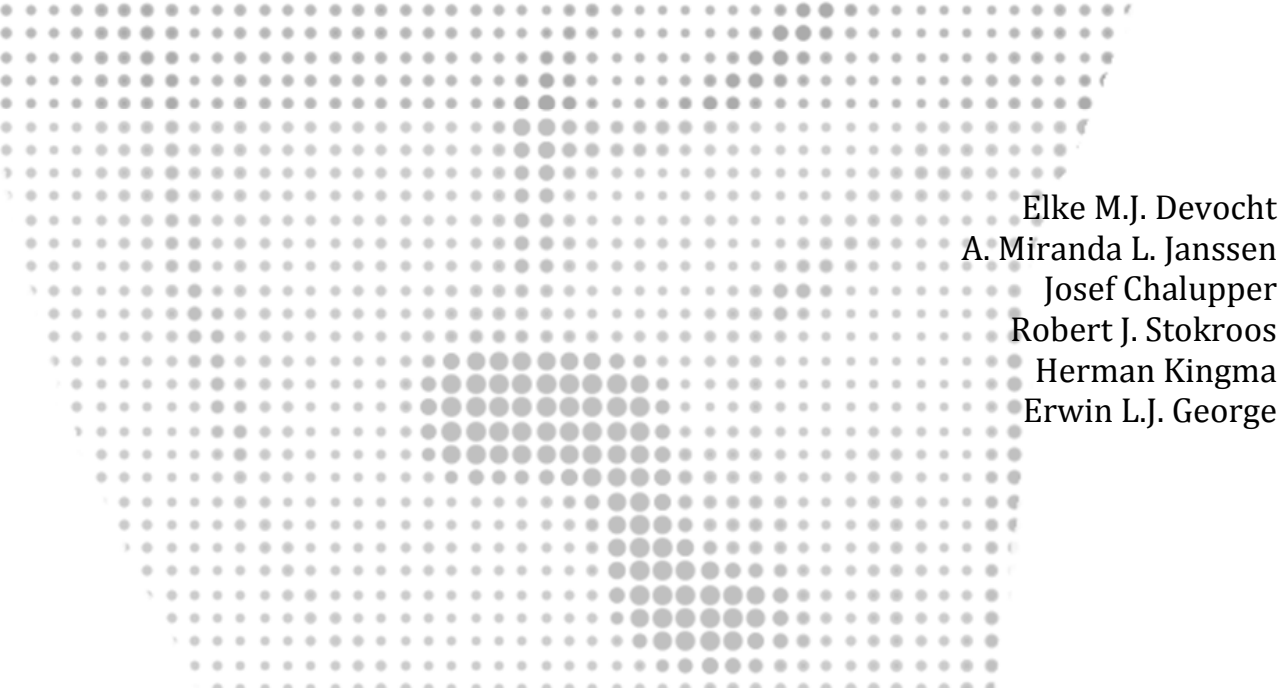
The objective of this study was to assess the subjective experiences of cochlear implant (CI) users either wearing or not wearing a hearing aid (HA) at the contralateral ear. A group of unilateral CI-recipients were asked to fill out a set of daily-life questionnaires on bimodal HA use, hearing disability, hearing handicap and general quality of life. Twenty-six CI-recipients regularly used a contralateral HA (bimodal group). The other twenty-two CI-recipients did not use a HA in the contralateral ear (unilateral group). Comparisons between both groups (bimodal versus unilateral) showed no difference in self-rated disability, hearing handicap or general quality of life. However within the group of bimodal listeners, participants did report a significant benefit of bimodal hearing ability in various daily life listening situations. It is shown that bimodal benefit in daily life can consistently be experienced and reported within the group of bimodal users. 


\section{INTRODUCTION}

Given the beneficial results of cochlear implantation (CI), candidacy criteria for receiving a $\mathrm{CI}$ have broadened from profound hearing loss to also include moderately-severe hearing loss (Gifford et al, 2010; Hughes et al, 2014; Leigh et al, 2016). As a result a growing number of CI recipients have aidable residual hearing in the contralateral ear (Fielden et al, 2016). In many countries around the world, current reimbursement regulations for adults support solely unilateral cochlear implantation. Fitting a contralateral hearing aid in the non-implanted ear when aidable residual hearing is present is generally recommended and well established as standard clinical practice (Ching et al, 2004; Offeciers et al, 2005; Scherf \& Arnold, 2014). Previous research has shown that an increasing number (50 to $60 \%$ ) of patients who receive a CI in one ear indeed prefer to retain their acoustic HA in the non-implanted ear (Cowan \& Chin-Lenn, 2004; Devocht et al, 2015; Lenarz et al, 2017).

Combining electrical stimulation by a $\mathrm{CI}$ in one ear with acoustic amplification by a conventional HA in the other ear, is known by the label of bimodal hearing. The benefits of bimodal hearing are attributed to the combined effects of the use of two ears (bilateral input), the opportunity to centrally combine the input in both ears (binaural cues), and the access to complementary information. Bilateral and bimodal effects such as summation, head shadow and squelch, are general characteristics of bilateral hearing (Dillon, 2012a). Moreover, bimodal hearing can offer the unique opportunity of combining complementary information by having access to two distinct modalities. Contralateral residual hearing is mainly situated in the low frequency region (Yehudai et al, 2012), which is known to contain cues regarding e.g. voice fundamental frequency, prosody and music (Crew et al, 2015; Most et al, 2012; Shpak et al, 2013; Zhang et al, 2010). Such information cannot be captured well by electrical stimulation. Literature shows that bimodal benefits can be demonstrated within the auditory domains of speech understanding in noisy situations, ease of listening, sound localization, music appreciation and sound quality (Ching et al, 2004; Ching et al, 2007; Cullington \& Zeng, 2011; Devocht et al, 2017; Gifford et al, 2007; Looi et al, 2007; Morera et al, 2012; Olson \& Shinn, 2008; Potts et al, 2009; Schafer et al, 2011; Sucher \& McDermott, 2009).

Despite the evidence of these bimodal advantages in research settings, the fact remains that about 40 to $50 \%$ of CI-recipients who are deemed eligible bimodal candidates, do not opt for the bimodal combination in daily life (Cowan \& ChinLenn, 2004; Devocht et al, 2015; Lenarz et al, 2017). Moreover, rather low correlations between objective performance measures and self-reported outcomes have been reported (Noble et al, 2008b). This is not surprising since a laboratory 
environment provides only a selected sample of abilities whereas self-ratings cover a generality of contexts in daily environments. Therefore, the self-assessed daily-life experiences of these patients are an important research area to address when investigating bimodal benefit.

By interviewing bimodal non-users, Fitzpatrick \& Leblanc (2010) identified three factors that primarily influenced the decision to discontinue hearing aid use, namely the experience with hearing aids prior to implantation, the percept of superiority of a unilateral CI compared to hearing aids, and the experienced interference with sound quality when a CI and hearing aid were combined. In a study conducted by Noble et al (2008a), results from self-report handicap questionnaires amongst different profiles of CI-users (CI+CI, CI alone, CI+HA) demonstrate a smaller handicap for the bilateral CI group compared to the other groups but no significant difference between the bimodal group and the group with only a unilateral CI. This could suggest that, in contrast to a second cochlear implant, the additional hearing aid does not contribute to reducing the handicap perceived by unilateral CI-patients. In fact the bimodal group even scored slightly higher handicap ratings compared to the unilateral group. A study examining CIrelated quality of life, however did show an improved rating of sound perception in bimodal compared to unilateral CI listeners (Farinetti et al, 2015). In contrast however, when assessing hearing disability ratings in specific daily life listening situations, the self-rated performance of a bimodal group was not statistically different from that of a unilateral CI group, in contrast to the significantly better performance of a bilaterally implanted group (Noble et al, 2008b). A more recent study repeated the earlier study in a comparable group of bimodal and a unilateral listeners using the same questionnaire (Farinetti et al, 2015). Even though scores seemed in favor of the bimodal users, again no statistical significant differences could be established between both groups, except for the scale of sound quality and naturalness. When bimodally fitted patients subsequently received a second cochlear implant however, disability scores using the same questionnaire, did improve significantly (Yawn et al, 2018).

Overall, questionnaire results so far seem to indicate that bimodal listeners experience only limited, if any, benefit over unilateral CI users. However in these studies only comparisons between groups were made. Up until now no study exists that looked into comparisons between the condition with and without their hearing aid within the group of bimodal users as to address the perceived level of benefit. 


\section{CURRENT STUDY}

Although many studies have proven the benefit of bimodal hearing in laboratory situations, there is still a lack of studies examining patient-related benefits of wearing bimodal devices in the real world (Farinetti et al, 2015). The current study aims to assess the experiences of recipients of a unilateral cochlear implant either wearing or not wearing a contralateral hearing aid by using a set of daily-life questionnaires in the field of bimodal use, hearing disability, hearing handicap and general quality of life. It was questioned whether comparisons between both groups could repeat the findings from previous literature (Fitzpatrick \& Leblanc, 2010; Noble et al, 2008a; Noble et al, 2008b). Additionally, it was examined whether bimodal benefits could be demonstrated by comparing listening conditions within the group of bimodal users.

\section{METHODS}

\section{ETHICS}

The local Medical Ethical Committee (Maastricht University Medical Center, NL42011.068.13) has approved this study as part of a larger clinical trial registered in the Dutch National Trial Register (NTR3932). The study has been conducted in accordance with the ethical principles as formulated in the Declaration of Helsinki. All participants have provided written informed consent to the inclusion of their anonymous data and received a small participation gift.

\section{PROCEDURE}

Inclusion criteria were that participants were capacitated adults, patients of CIteam South-East Netherlands, users of a unilateral CI of the brand Advanced Bionics $^{\mathrm{TM}}$ (AB) (Valencia, USA), had at least one year of CI experience, used the CI speech processor more than 10 hours a day, were willing and able to fill out questionnaires and agreed to participate in the study by informed consent. Subjects were excluded if they were less than 18 years of age or incapacitated, were non-Dutch speaking or used bilateral cochlear implants.

All subjects that were deemed eligible, were invited by mail. They were requested to fill out the paper-based questionnaires and return them together with the informed consent form in order to participate. If no response, either positive or negative, was received within one month, a non-committal reminder was sent. When some of the responses of participating subjects were ascertained to be missing within or across questionnaires, a one-time request for clarification and addition was sent in order to complete the data collection. 


\section{QUESTIONNARES}

A set of five self-administering questionnaires was compiled to assess daily-life experiences of unilateral $\mathrm{CI}$ recipients regarding their bimodal hearing aid use, hearing (dis)abilities, hearing handicap and health related quality of life (HRQL).

\section{BIMODAL USE}

In order to explore the use of the bimodal hearing combination, a composite bimodal questionnaire was used. The bimodal questionnaire was designed in line with the questionnaire recently used by Neuman et al. (2017). It represents a fusion of questions derived from four existing questionnaires: the bimodal questionnaires by Fitzpatrick et al. (2009), Fitzpatrick \& Leblanc (2010) and Tyler et al. (2002), and the International Outcome Inventory for Hearing Aids (IOI-HA) by Cox et al. (2000). The resulting explorative questionnaire and its composition can be found in Appendix 1. Questions were formulated in Dutch and divided in three main parts: the experience with HAs prior to receiving the CI (7 items), the decision-making process on retaining the contralateral HA (3 items) and the experiences with a contralateral $\mathrm{HA}$ after CI implantation (29 items). The main themes that were addressed by a combination of open and closed questions are: the frequency of HA use, the situations in which bimodal stimulation is preferred, the fitting of CI and HA settings, the balance/fusion of CI and HA, the satisfaction with the HA and the decision of (dis)continued HA-use. The bimodal questionnaire was composed to be applicable to all unilateral CI-recipients whether they still wore a contralateral HA, tried one but stopped using it or did not try a contralateral $\mathrm{HA}$ at all. In the latter case a number of questions could be skipped in order to fit the patient-related situation.

HEARING [DIS]ABILITY

Two relevant questionnaires were used to measure the patients' perception of residual hearing disability in daily-life listening situations: the SSQ and the AVETA. The SSQ, the Speech, Spatial and Qualities of hearing scale, was used in its Dutch version (Demeester et al, 2012). This questionnaire is designed and validated by Gatehouse \& Noble (2004) to reflect real life listening experiences of patients with different hearing profiles and rehabilitative interventions such as hearing aids and cochlear implants (Noble et al, 2008b; Noble \& Gatehouse, 2006). The SSQ is designed with a special focus on daily listening situations whereby binaural auditory functions play an important role such as understanding speech in complex situations and localizing environmental sounds. The SSQ consists of 49 questions, divided in three main scales, asking subjects to respond on a visual analog scale of 0 (not at all) to 10 (perfectly). The Speech scale (14 items) asks to 
rate the ability to understand speech under quiet and noisy conditions as well as in more complex situations of speech perception. The Spatial scale (17 items) questions the performance to localize sounds and judge the distance of moving objects. Finally, the Qualities scale (18 items) aims to map the identification and segregation of sounds as well as the naturalness and effort of listening. In total, these substantives subdomains make that the questionnaire can also be described by 10 pragmatic subscales (Gatehouse \& Akeroyd, 2006).

Secondly, the AVETA, Amsterdam Questionnaire for Unilateral or Bilateral Fittings (Boymans et al, 2008), is a Dutch specialized questionnaire that combines two existing questionnaires: the Amsterdam Inventory of Auditory Disability and Handicap (Kramer et al, 1995) and the Abbreviated Profile of Hearing Aid Benefit (Cox \& Alexander, 1995). Originally the AVETA also included questions from the IOI-HA (Cox et al, 2000), however to avoid overlap with the questionnaire on bimodal use only the 18 main questions of the AVETA were used. The questionnaire covers six categories: detection of sounds (3 items), discrimination or recognition of sounds (3 items), speech intelligibility in quiet (3 items), speech intelligibility in noise (3 items), directional hearing and comfort of loud sounds (3 items). Subjects were asked to respond by checking one of four answering alternatives (never, sometimes, often, almost always).

For both hearing (dis)ability questionnaires subjects were asked to respond to each question as fitted with their daily hearing device or devices. Those subjects who continued to regularly use a contralateral HA besides the CI were asked not only to fill out each question for the bimodal condition but to also respond for the listening condition with $\mathrm{CI}$ alone and with HA alone in order to reflect the perceived added value of each device besides the other.

HEARING HANDICAP

According to the World Health Organization (World Health Organisation, 1980), handicap is defined as a disadvantage for a given individual, resulting from an impairment or a disability, that limits or prevents the fulfillment of a role that is normal for that individual. Handicap as such represents the continued socialization of an impairment or disability. To assess whether wearing a contralateral hearing aid affects the hearing specific perceived handicap of unilateral CI patients, the Hearing Handicap Questionnaire (HHQ) was used. The questionnaire was developed by Gatehouse and Noble (2004) aside from the SSQ questionnaire and has been validated among CI recipients (Noble et al, 2008a) as a useful questionnaire of the true concept of handicap as defined by the World Health Organisation (1980). The original English questionnaire was translated and backtranslated into Dutch by a native English audiologist with a good level of Dutch and 
two native Dutch speakers with a Master's degree in English. The 12 questions of the HHQ can be resolved into two unique factors: emotional distress (7 items) and social restriction (5 items). Subjects were asked to respond by checking one of five answering alternatives (never, rarely, sometimes, often, almost always) representing their daily perceived handicap as fitted with their hearing device(s).

\section{HEALTH RELATED QUALITY OF LIFE}

Hearing loss is known to affect the ability to exchange information and therefore affecting a person's quality of life (Dalton et al, 2003). In this study the Dutch version of the HUI3, Health Utility Index Mark III, was used to assess HRQL (Grutters et al, 2007). The questionnaire is a generic multi attribute preferencebased measure of health status and HRQL that is widely used as an outcome measure in clinical studies (Feeny et al, 2002). Subjects were asked to check their daily perceived level of health with respect to 8 attributes: vision, hearing, speech, ambulation, dexterity, emotion, cognition and pain/discomfort. The levels within each attribute vary from highly impaired or disabled to normal. The attribute level per health domain was determined and consequently translated to the multiattribute level utility score whereby each attribute is considered in relation to the other health domains. Finally, by applying health related weights and combining scores across all attributes, the resulting total score of HRQL on a scale of 0 ( = death) to 1 ( = full health) is calculated. The HUI3 has been put forward as being the instrument of first choice when measuring utility within a population with hearing complaints (Grutters et al, 2007) and in CI-recipients (Ramakers et al, 2016)

\section{Participants}

Invites were sent out to 77 patients between April 2013 and March 2014. 48 subjects were included in the study by completing the questionnaires. 29 subjects were not included due to reported or unreported non-response or incomplete informed consent. The response rate in this study (62\%) is comparable to other questionnaire studies (Farinetti et al, 2015). 26/48 subjects reported to regularly use ( $>50 \%$ of time) a conventional hearing aid in the contralateral ear (bimodal group), while the other 22/48 subjects only used the unilateral CI (unilateral group). Table 1 presents the mean characteristics and hearing history of patients in both groups alongside their last audiometric results within one year around the time of study involvement. Hearing history and other demographic information appeared not to differ between both groups. The residual hearing in the non-implanted ear was found to be significantly different ( $p<0.05$, independent-samples Student t-test) between both groups. In the current study 
the bimodal group showed more favorable hearing thresholds up to $1000 \mathrm{~Hz}$ compared to the unilateral group (Figure 1).

TABLE 1. Patient characteristics of bimodal and unilateral subjects.

\begin{tabular}{lrrrrc} 
Variable & \multicolumn{2}{c}{$\begin{array}{c}\text { Bimodal } \\
(\mathrm{n}=26)\end{array}$} & $\begin{array}{c}\text { Unilateral } \\
(\mathrm{n}=22)\end{array}$ & $\begin{array}{c}\mathrm{p} \\
\text { Sex (male/female,n) }\end{array}$ \\
Age (years) & $11 / 15$ & $15 / 7$ & 0.089 \\
Duration of deafness & 63.9 & $(2.7)$ & 67.2 & $(3.0)$ & 0.406 \\
pre-implantation (years) & 38.8 & $(3.2)$ & 32.1 & $(3.5)$ & 0.164 \\
Age onset deafness (years) & 21.7 & $(4.3)$ & 30.8 & $(5.0)$ & 0.173 \\
CI-experience (years) & 3.6 & $(0.4)$ & 4.3 & $(0.4)$ & 0.206 \\
Residual hearing (PTA, dB HL) & 96.6 & $(2.7)$ & 107.6 & $(3.8)$ & $0.020^{*}$ \\
CNC FITTED (\%) & 70.4 & $(4.0)$ & 62.6 & $(6.7)$ & 0.323 \\
CNC CI alone (\%) & 58.7 & $(4.4)$ & - & - & - \\
CNC HA alone (\%) & 46.3 & $(4.4)$ & & -
\end{tabular}

Patient characteristics of bimodal and unilateral subjects: mean (standard error) per group and significance of difference between groups $(* \mathrm{p}<0.05)$ based on Fisher's exact test for the variable 'sex' and independent-samples Student T-tests for other variables. $\mathrm{CI}=$ cochlear implant, $\mathrm{HA}=$ hearing aid, FITTED $=$ referring to CIHA in case of bimodal and $\mathrm{CI}$ in case of unilateral, $\mathrm{CNC}=$ consonant-nucleus-consonant maximum phoneme score across 55-65-75 $\mathrm{dB}$ SPL in quiet free-field, PTA = pure-tone average across $0.5,1$ and $2 \mathrm{kHz}$ under headphones in the unaided contralateral ear.

FIGURE 1. Residual hearing.

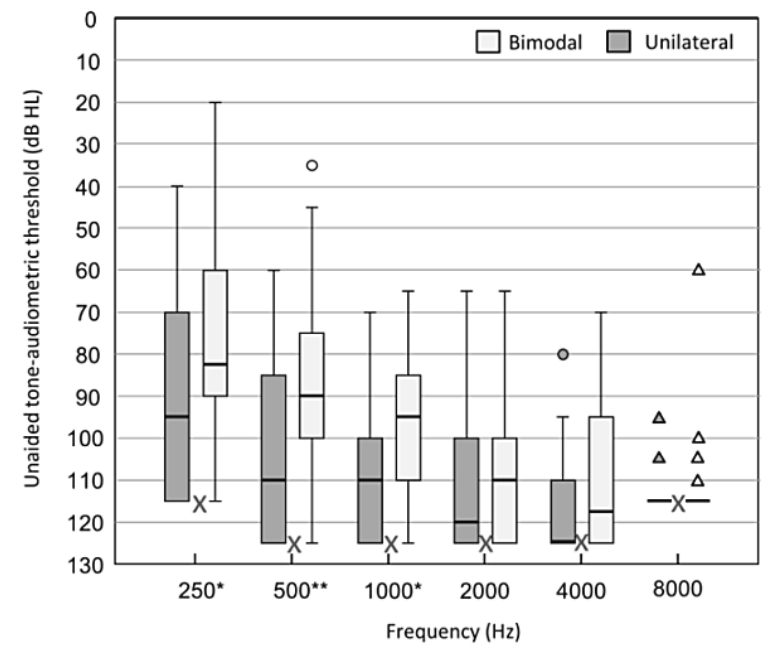

Unaided pure-tone thresholds in the non-implanted ear for bimodal $(n=26)$ and unilateral $(n=22)$ subjects. If no response could be recorded within the limits of the audiometer, a value of $5 \mathrm{~dB}$ HL greater than the maximum tested level was entered (see X markings). Box plots represent the distribution per frequency (median and interquartile range), with whiskers denoting minimum and maximum values within 1.5 times the interquartile range, circles denoting outliers and triangles denoting extremes. Significant differences between groups are based on independentsamples Student T-tests per frequency $\left({ }^{*} \mathrm{p}<0.05,{ }^{* *} \mathrm{p}<0.01\right)$. 


\section{DATA ANALYSIS}

Data were digitally entered by two independent persons using an excel file designed to avoid invalid entries. The final data file arose by merging both entry versions and correcting for discrepancies. The data of the explorative questionnaire on bimodal use were handled descriptively. The data of the other questionnaires were analyzed statistically after dealing with missing values. Statistics were performed using IBM SPSS Statistics version 21.0.0.1

Missing values were handled per questionnaire and per group. Cases were included if more than 90 percent of the questionnaire items was completed. Bimodal cases, when measured under three listening conditions (CI, HA, CIHA), were included if they had more than 90 percent complete data in at least one listening condition and up to a maximum of one listening condition without any item response. In the resulting dataset missing responses on questionnaire items were replaced using multiple imputation (MI) to generate 100 complete datasets. These datasets were then analyzed separately, and finally a single (pooled) MI estimate and its standard error was calculated by combining the estimates and standard errors obtained from each completed dataset using 'Rubin's rules' (Little \& Rubin, 2002; Rubin, 1987). The imputation method was 'Fully Conditional Specification' or 'Monotone' as determined by the automatic method in SPSS's module for multiple imputation. To improve the accuracy of the imputed values (Graham, 2009), next to questionnaire items the imputation model also included participants characteristics (Table 1) as auxiliary variables. Missing item responses were modeled by linear regression.

To compare outcomes between groups parametric two-tailed independent samples student $t$-tests were conducted for the listening condition 'as fitted', referring to $\mathrm{CI}$ alone in case of the unilateral group and CI and HA together for the bimodal group. When analyzing the hearing (dis)ability questionnaires (SSQ and AVETA) as to compare the three listening conditions within the bimodal group (CI, HA and CIHA), linear mixed model analyses were performed with person as random factor and listening condition as fixed factor. To select the most appropriate covariance structure (an unstructured matrix or a compound symmetry matrix), the restricted maximum likelihood ratio test was used. When a significant main effect was found, the three listening conditions (CI, HA and CIHA) were all compared pairwise and Bonferroni adjusted p-values were considered. Statistical significance is defined as a p-value of $<0.05$. 


\section{RESULTS}

\section{BIMODAL USE}

The complete questionnaire on bimodal use can be consulted in Appendix 1. The absolute and relative frequency at which each answering alternative is chosen, are presented per question. Furthermore results are visually summarized as compared between the unilateral and the bimodal users. A descriptive overview of the main findings is presented here.

In both groups more than $60 \%$ of the participants used to regularly ( $>10$ hours a day) wear a conventional HA in both ears prior to receiving their cochlear implant (Q3). The degree of bilateral HA experience therefore does not seem to be related to whether subjects either retain or reject the contralateral hearing aid after implantation. The quality of hearing with the conventional hearing aids however does appear to differ between both groups (Q6). Most of the bimodal users (56\%) experienced their preoperative HAs as being very helpful, while a large group of unilateral CI users (36\%) described their HAs as rarely being helpful. This is in line with the finding that most of the bimodal users (65\%) reported the prior intention of retaining the hearing aid aside the $\mathrm{CI}$, while most unilateral users (68\%) had not yet made up their mind before surgery on retaining a contralateral HA(Q8). As a result, $77 \%$ of subjects in the bimodal group, compared to only $27 \%$ in the unilateral group, started using the HA directly after CI surgery (Q15). In contrast, half of the subjects in the unilateral group never tried a contralateral HA after receiving their $\mathrm{CI}$ and the majority of unilateral subjects who did try a HA (78\%) reported that the sound from both sides didn't fuse to become one image (Q34). Overall the use of a contralateral HA augmented (73\%) the personal enjoyment in life for bimodal subjects while it made no difference $(56 \%)$ or resulted in a degradation (33\%) for subjects in the unilateral group (Q37).

\section{HEARING [DIS]ABILITY}

\section{SSQ}

Two unilateral and two bimodal cases were excluded from the analysis of the SSQ questionnaire due to more than $10 \%$ missing values. The pooled overall score and the scores per main scale of the SSQ questionnaire are presented in Figure 2. Details on the mean scores of the ten pragmatic subscales of the SSQ questionnaire can be consulted in Appendix 2.

Results showed no significant difference of the overall score or any of the (sub)scales between the unilateral and the bimodal group (as fitted). Within the 
bimodal group however, different listening conditions (CI, HA, CIHA) produced significantly different scores $(\mathrm{F}(2,23) ; \mathrm{p}<0.001)$. Bimodal subjects rated the daily life performance of the combination of $\mathrm{CI}$ and HA together significantly higher $(\mathrm{p}<$ 0.01) than the performance of the CI (about 1 point) or the HA only (about 2 points). This finding was consistent across all daily auditory functioning situations as represented by scoring the different (sub)scales. Although most (sub)scales did not demonstrate a difference between performance with $\mathrm{CI}$ and HA, the overall SSQ outcome did show that the hearing ability with CI alone was rated significantly better than the ability with HA alone $(\mathrm{p}<0.05)$.

FIGURE 2. SSQ.

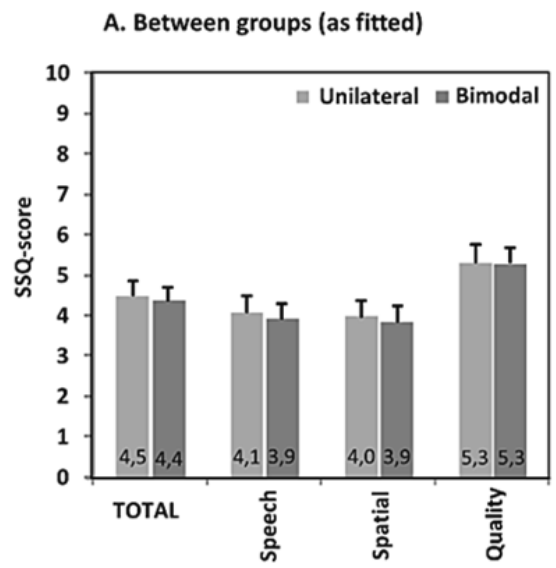

B. Within bimodal group

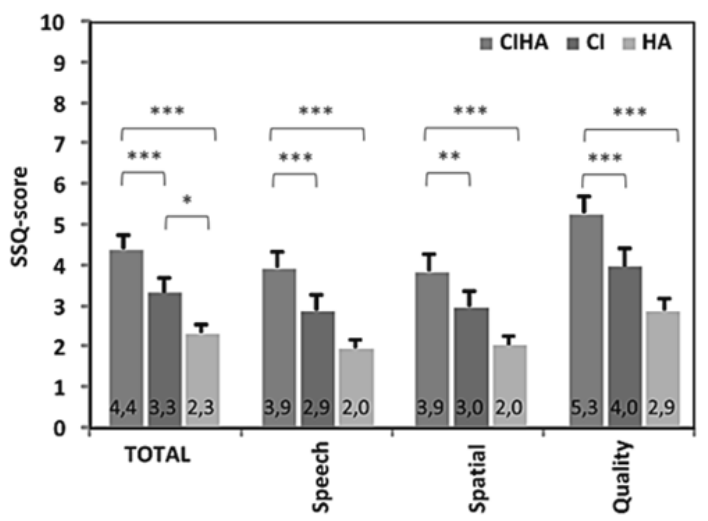

Hearing (dis)ability ratings for subjects who only use a cochlear implant (CI) (unilateral group, $\mathrm{n}=20$ ) and subjects who also use a hearing aid (HA) in the non-implanted ear (bimodal group, $n=24)$. Pooled mean (+standard error) overall scores (SSQ) and scores per main scale (Speech, Spatial, Quality) on a visual-analogue scale (VAS, 0-10) using the SSQ-questionnaire by Gatehouse \& Noble (2004). Scores were compared between groups as fitted (A.) and evaluated within the bimodal group for listening conditions with CI, with HA and with CI and HA together (B.) Asterisks denote significant differences between groups or listening conditions $\left({ }^{*} \mathrm{p}<0.05,{ }^{* *} \mathrm{p}<0.01,{ }^{* * *} \mathrm{p}<0.001\right)$. 


\section{AVETA}

Three unilateral and four bimodal subjects were not included in the final analysis of the AVETA questionnaire since more than $10 \%$ of their data were established to be missing. The pooled overall score and the scores per category on the AVETA questionnaire are presented in Figure 3.

FIGURE 3. AVETA.

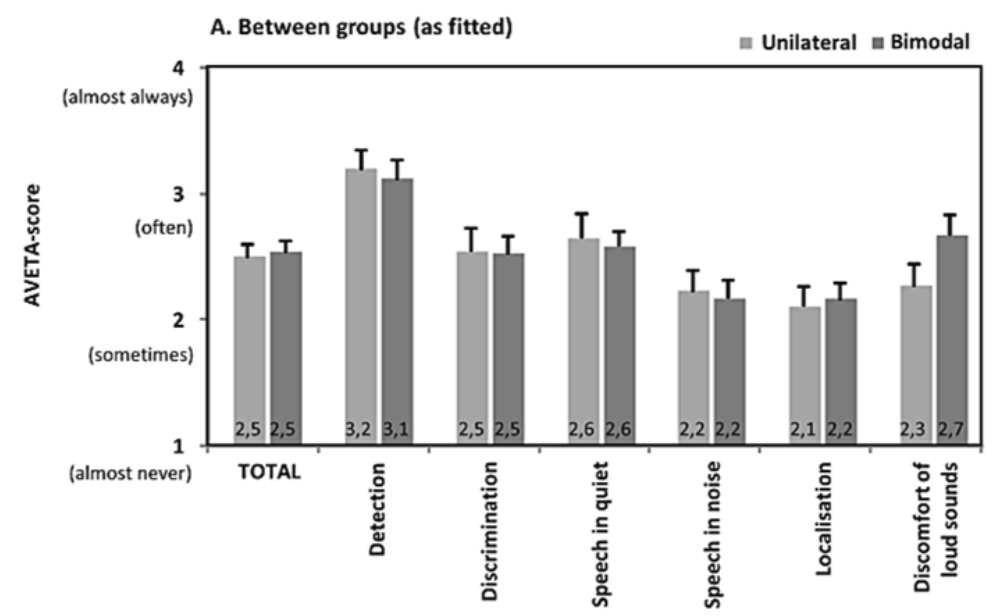

B. Within bimodal group

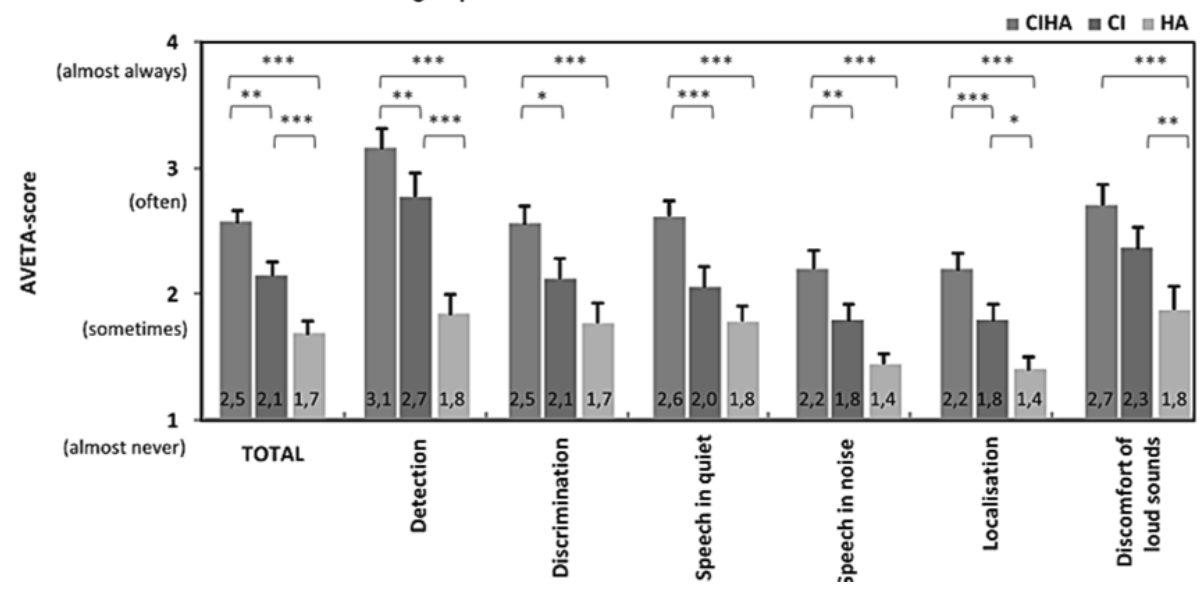

Hearing (dis)ability ratings for subjects who only use a cochlear implant (CI) (unilateral group, $\mathrm{n}=19$ ) and subjects who also use a hearing aid (HA) in the non-implanted ear (bimodal group, $n=22$ ). Pooled mean (+standard error) overall score and scores per category using the AVETA-questionnaire by Boymans et al (2008). Scores were compared between groups as fitted (A.) and evaluated within the bimodal group for listening conditions with $\mathrm{CI}$, with $\mathrm{HA}$ and with $\mathrm{CI}$ and HA together (B.) Asterisks denote significant differences between groups or listening conditions $\left({ }^{*} \mathrm{p}<\right.$ $\left.0.05,{ }^{* *} \mathrm{p}<0.01, * * * \mathrm{p}<0.001\right)$. 
Although a slightly higher level of discomfort for loud sounds was seen in the bimodal group compared to the unilateral group, no significant difference was found between both the unilateral and the bimodal group on the overall score or on any of the other subscales. For subjects within the bimodal group however, a significant effect of listening conditions (CI, HA, CIHA) was found $(\mathrm{F}(2,42)$; $\mathrm{p}<0.001$ ). Listening with the bimodal combination (CIHA) was rated significantly higher compared to the CI or the HA alone $(\mathrm{p}<0.05)$ in all questioned auditory situations except in the discomfort scale. The performance with CI alone was rated significantly better compared to HA alone for the overall scale as well as for the detection and the localization scale $(\mathrm{p}<0.05)$. The discomfort level for loud sounds was rated significantly higher (it is less favorable) $(\mathrm{p}<0.05)$ for the bimodal combination compared to the $\mathrm{HA}$ alone condition. The $\mathrm{CI}$ alone condition in its turn was experienced to be less comfortable $(\mathrm{p}<0.05)$ than the HA alone condition.

\section{HEARING HANDICAP}

All cases were included in the analysis of the HHQ questionnaire and no imputations were performed since no missing values occurred. Results of the HHQ questionnaire are presented in Figure 4.

FIGURE 4. HHQ.

Between groups (as fitted)

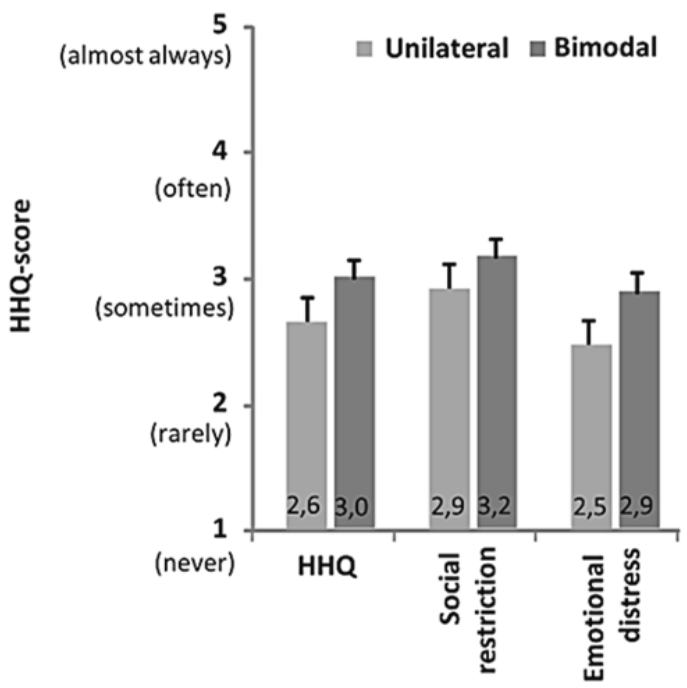

Hearing handicap ratings for subjects who only use a cochlear implant (CI) (unilateral group, $\mathrm{n}=22$ ) and subjects who also use a hearing aid (HA) in the non-implanted ear (bimodal group, $n=26$ ). The mean (+standard error) overall score and scores per subscale using the HHQ-questionnaire by Gatehouse and Noble (2004). No significant differences between groups (as fitted) were found ( $\mathrm{p}>0.05)$. 
Although ratings in the bimodal group were higher for the overall outcome as well as on both subscales, suggesting a slightly higher level of perceived handicap compared to the unilaterally fitted subjects, scores were not found to be significantly different between both groups.

HEALTH RELATED QOL

One of the unilateral cases and none of the bimodal cases were discarded from the final analysis due to more than $10 \%$ missing values. The pooled multi-attribute utility score per attribute as well as the pooled overall HRQL score is presented in Figure 5.

The attribute vision showed a significant difference between both groups $(\mathrm{p}<0.05)$, whereby the visual functionality in the bimodal group was scored less optimal compared to the unilateral group. No significant differences were found between both groups in any of the other health domains, including the hearing domain. It can be noticed that all health domains scored on average a perfect or near perfect attribute level, except for the hearing domain reflecting the population of hearing

FIGURE 5 . HUI3.

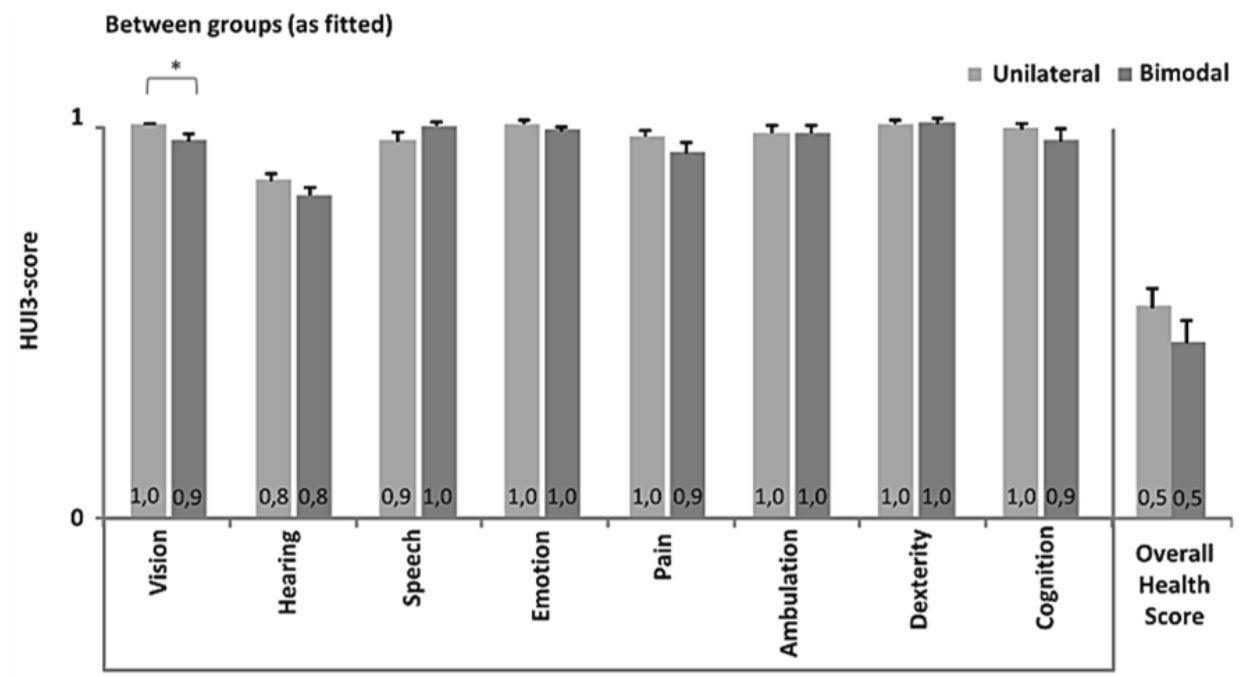

Multi Attribute Utility Score

Health related Quality of Life ratings for subjects who only use a cochlear implant (CI) (unilateral group, $\mathrm{n}=21$ ) and subjects who also use a hearing aid (HA) in the non-implanted ear (bimodal group, $n=26)$. Pooled mean $(+$ standard error) multi attribute utility scores across health related subscales and the pooled mean (+standard error) overall health utility score are determined using the HUI3 questionnaire by Grutters et al (2007). Asterisks denote significant differences between both groups $(* \mathrm{p}<0.05)$. 
impaired subjects in the use of their hearing equipment. Although a slightly lower score was found in the bimodal group, no significant difference could be established between both groups looking at the overall HRQL.

\section{DISCUSSION}

\section{EXPERIENCE WITH BIMODAL AIDNG}

The aim of the current study was to assess the experiences of CI recipients either wearing or not wearing a contralateral hearing aid. The study sample consisted for $54 \%$ of bimodal subjects which seems a good representation of the target group, given that a bimodal retention rate of $64 \%$ was previously established within a larger sample of the CI population in the same CI center (Devocht et al, 2015). The experience of using the bimodal combination was examined by a qualitative questionnaire on bimodal use. Results seemed to indicate that it is not the degree of hearing aid experience but rather the functional quality of the hearing aid experience prior to implantation that differed between the bimodal and the unilateral group. A study by Fitzpatrick \& Leblanc (2010) previously suggested a comparable conclusion. This finding can be interpreted in the light of the fact that demographic information and hearing history did not differ between participants in both groups. However, the degree of residual hearing in the contralateral ear was found to significantly differ between both groups, whereby the bimodal group demonstrated better residual hearing thresholds in the low frequencies compared to the unilateral group. These findings are consistent with previous reports in bimodal literature (Farinetti et al, 2015). Indeed a database study by Devocht et al (2015) also demonstrated that the rate of retaining a hearing aid in the contralateral ear one year after receiving a unilateral CI, was not significantly related to demographics or hearing history. Instead, the retention rate was related to the amount of residual hearing, to the residual speech understanding score and to the difference in scores between the HA and the CI ear. The perceptual difference between both ears can in turn be linked to the current finding that a number of unilateral users reported an unfused sound image when trying the CI together with a HA.

Despite the given negative experiences of unilateral users who did try a contralateral HA, it should be noted that more than $50 \%$ never again tried a HA after receiving their CI. It is therefore possible that a potential group of bimodal users was missed by never giving the HA a chance. Therefore, clinicians have an essential role to provide patients with the tailored fitting of the appropriate devices. When counseling eligible CI-candidates in clinical practice, it is important to identify those subjects that would make good bimodal users. Although the 
criteria for bimodal candidacy are still unresolved, previous research did present discrimination values to identify which unilateral CI patients are most likely to turn into bimodal users (Devocht et al, 2015).

\section{COMPARING BIMODAL AND UNILATERAL USERS}

Daily life experiences of unilateral and bimodal CI users were measured by a set of self-administrative questionnaires, taking into account the different aspects of hearing impairment. To assess a subjects' personal health experience it is important to not only question a functional disability resulting from a physical impairment, but also ask about the social and cultural consequences of the impairment (handicap) as well as the overall health quality as perceived by the concerned individual (Barbotte et al, 2001; World Health Organisation, 1980).

When comparing the outcomes 'as fitted' between those CI recipients who do (bimodal group) and those who do not (unilateral group) wear a contralateral HA, no significant differences could be found across scales concerning disability or handicap, which is in line with previous findings by Noble et al (2008b). Another study (Farinetti et al, 2015) did find higher scores for the bimodal compared to the unilateral group, however statistical significance could not be reached except for the SSQ-subscale of sound quality and naturalness. Consistent with these findings, current results showed that bimodal users rated this particular Quality subscale higher compared to the unilateral group. However, statistical significance could not be reached in the current sample. Other research has indeed demonstrated that sound quality is enriched by adding a HA aside a unilateral CI (Devocht et al, 2017).

The overall health related quality of life was not found to be significantly different between both groups. Only the attribute level of vision was established to just significantly differ between both groups whereby the vision state of the bimodal users was less optimal compared to the unilateral listeners. A possible reason for this finding might be that a reduced visual quality urges to compensate by optimizing other sensory modalities, increasing the chance of wearing a contralateral HA. Low frequency acoustic hearing provided by a HA namely is known to improve the representation of voicing (Zhang et al, 2010) which can compensate for reduced visual lip-reading abilities. Also, bimodal input can to some extent enable sound localization (Ching et al, 2007) which can make up for a diminished visual orientation. However, no existing literature was found to support the current finding of a reduced vision state in bimodal users, indicating future research is warranted to investigate whether this finding is repeatable. 


\section{Percelved BIMOdAL BeneFIT}

The current study also questioned, to our knowledge for the first time, the differences in disability between the listening conditions with CI alone, HA alone and $\mathrm{CI}$ and HA together within the group of bimodal users. Results showed that the bimodal combination of CI and HA together was rated consistently more favorable across all questioned daily life listening situations, except for discomfort of loud sounds. The aversiveness of loud sounds was rated higher, that is less favorable, for the bimodal listening condition compared to the condition with HA alone or CI alone, even though this last comparison failed to reach significance. This finding can be explained in the light of the binaural loudness summation effect and is consistent with outcomes for bilateral conventional hearing aids collected using the same AVETA-questionnaire (Boymans, 2003). Giving these results it is important to include the restoration of normal loudness perception in the fitting process of hearing equipment. It has been demonstrated that loudness normalization especially of binaural broadband signals asks for individual gain corrections which cannot always be corrected for by compression algorithms (Oetting et al, 2017).

The observation that bimodal benefit was present across all other questioned listening situations, shows that bimodal hearing cannot only be beneficial in complex situations reflecting true binaural hearing capacities, but also means an improvement for basic auditory functions (Farinetti et al, 2015) like identifying sounds and understanding speech in quiet. It has been confirmed that bimodal aiding indeed enhances multiple dimensions of speech perception such as intelligibility, listening effort and sound quality, whereby the bimodal summation effect and the access to complementary information is believed to play an important role (Devocht et al, 2017) .

It is known that the degree of bimodal benefit in laboratory settings shows a large amount of variability among bimodal listeners (Devocht et al, 2017; Schafer et al, 2011). The origin behind the degree of benefit is still unclear. The chance of a unilateral CI-recipient becoming a bimodal user, is known to be related to the level of residual hearing (Devocht et al, 2015; Farinetti et al, 2015). Meanwhile, the actual degree of bimodal benefit cannot be explained by the amount of residual hearing alone (Devocht et al, 2017; Dincer D'Alessandro et al, 2015; Veugen et al, 2015; Yoon et al, 2012a). For example, it is known that the fitting of the HA may play an important role (Harris \& Hay-McCutcheon, 2010; Yehudai et al, 2013). In the current study, the fitting of the CI and the HA were not assessed directly. Rather, the data show how subjects evaluated their daily life functioning by using their hearing devices in the daily settings. All subjects were active patients at the CI center of MUMC+ and had regular check-ups of their devices when necessary. In 
clinical practice, as in many CI centers around the world, no systematic bimodal fitting protocol was applied, since no generally accepted bimodal fitting method exists (Scherf \& Arnold, 2014). Furthermore other factors such as the spectral resolution ability of the contralateral ear (Zhang et al, 2013) as well as the difference in speech recognition abilities between both ears (Yoon et al, 2015) have been suggested as playing a role in the measured bimodal benefit. The small sample sizes of most CI studies (e.g. Devocht et al, 2017) however often limit the ability to investigate correlations between related factors with sufficient statistical power. Combining findings by meta-analysis into larger samples and prospective follow-up of subjects whereby patients act as their own control, should therefore be considered in future research.

When looking at the degree of favorable bimodal benefit in the current study an average amount of 0.6 to 1.9 points was seen across all SSQ-scales using a scaling from 0 to 10 . For the AVETA-questionnaire a mean benefit of 0.3 to 0.6 was observed with a scaling from 1 to 4 . The established degree of bimodal benefit lies in range with an average SSQ-score difference of 0.8 to 2.0 that is reported for subjects transferring from one CI to a successive second CI (Summerfield et al, 2006; Yawn et al, 2018). Even though the amount of bimodal and bilateral benefit seems quite similar, the resulting ability level is known to be significantly more favorable for the bilaterally implanted subjects compared to bimodal or unilateral CI users (Noble et al, 2008b). The question then arises as to what makes a bimodal and a unilateral CI-recipient different, and yet results in a rather comparable disability outcome 'as fitted'? It has been hypothesized before that listeners choosing a bimodal fit might experience tougher demands in their daily life activities and may have not been performing well with the $\mathrm{CI}$ alone, giving rise to a continued HA use in an effort to improve things (Noble et al, 2008b). Bimodal subjects indeed rated their ability level with $\mathrm{CI}$ alone less optimal compared to the unilateral group. However, no significant difference in CNC-score with CI-alone was observed between both groups. Overall the bimodal group scored slightly better for the condition 'as fitted'. Earlier research indeed showed that the CNCscore with CI alone is in itself not related to bimodal HA retention (Devocht et al, 2015). There is no doubt however that daily auditory functioning is much more complex than illustrated by testing word scoring in quiet. It should therefore be noted that current results also demonstrated that, even though not significant, the bimodal subjects on average scored their residual hearing handicap about 0.4 points higher (that is worse) on a scale of 0 to 5 compared to the unilateral CI users. This is in line with previous findings done by Noble et al (2008a). In the light of these handicap ratings, it could be that the personal expectations and environmental requirements related to daily auditory functioning, are situated at a higher performance level within the group of bimodal subjects compared to the 
unilateral group. Further research into this social field of bimodal hearing is warranted in order to test this hypothesis.

\section{MethOdOLOGICAL CONSIDERATIONS}

Since missing data are a familiar phenomenon in a survey study, efforts were undertaken to complete the data collection. For the SSQ and AVETA survey it was observed that missing items mainly occurred for the CI and HA alone conditions within the group of bimodal users. This might be due to the fact that most bimodal subjects wore the CIHA combination during most of the day, making it more difficult to judge unilateral listening conditions in itself. This is an important limitation of the current study set-up which by all known means could not be avoided. Bimodal subjects who at first did not respond to some unilateral conditions were therefore provided with the extra instructions to complete all conditions in order to reflect the added value of one device versus the other when listening across all questioned daily life situations.

Despite all efforts, however a portion of the data remained missing. Cases marked by more than $10 \%$ missing values were removed from the final analysis per questionnaire. As a result the sample size varied slightly across quantitative questionnaires. The amount of excluded cases fortunately remained limited and was comparable in the bimodal and unilateral group. Since no direct evidence was found to establish the origin of the missing values in the remaining cases, missing values were dealt with by means of multiple imputation taking demographic information into account. This method is especially designed for complex cases where theoretically satisfactory answers are difficult to derive explicitly and has proven its validity under the assumption of ignorable non response (Rubin, 1987).

A survey study is known to be subjective in nature since it questions the personal experiences of subjects. By rating daily listening situations responses are made against an internal point of reference. When comparing hearing devices it is not possible to treat the conditions blindly. Especially in the case of bimodal subjects when questioning bimodal benefit by comparing the listening conditions with $\mathrm{CI}$, HA and CIHA, a bias towards the most used and preferred bimodal situation cannot be ruled out. It is therefore important to realize that the observed difference between the bimodal and the unilateral conditions should not be seen as a quantification of the actual measurable benefit but as the reflection of the perceived merit driving bimodal subjects to opt for the bimodal combination. 


\section{USED QUESTIONNAIRES}

Disability outcomes were collected using two questionnaires. The SSQ questionnaire is a well-established tool within the field of hearing rehabilitation and is available in multiple languages. With its 49 questions it is however a quite extensive survey to complete, making it not very suitable for clinical practice. The AVETA on the other hand only consists of 18 illustrated questions with confined answering alternatives. The survey has been developed with the same basic intentions as the SSQ, but is on the other hand only available in Dutch language. The current study illustrates that comparable results were achieved by using both questionnaires within the field of bimodal aiding. Since the start of the current study, a new version of the SSQ questionnaire, namely the SSQ12, has been proposed (Noble et al, 2013). This reduced form of the SSQ-questionnaire is especially developed for clinical use. In this light current data were also evaluated on the proposed 12 items only (not displayed). Since the perceived bimodal benefit was present across all questioned listening situations, the SSQ12 outcome yielded comparable results compared to the full SSQ survey. Based on these findings it can be advised to use the SSQ12 survey when evaluating bimodal disabilities in a clinical setting.

The HUI3 is a general QoL questionnaire that is frequently used in and presented as the preferred QoL survey regarding hearing related research (Grutters et al, 2007; Maes et al, 2011; Ramakers et al, 2016). It should however be remarked that the questions reflecting the hearing item in itself are very general in nature and do not differentiate between hearing devices (e.g. hearing aid, cochlear implant, etc). This can lead to confusions especially in the case of bimodally fitted subjects. The cost-effectiveness of bimodal hearing devices versus bilateral implantation is gaining more and more interest these years (Smulders et al, 2016; Yawn et al, 2018). Therefore, it is important to use a QoL measure that is sufficiently sensitive to changes in hearing related health state, specifically concerning various listening conditions, as shown by the difference in daily life results within the current study's bimodal group.

\section{CONCLUSION}

When investigating bimodal benefit, the self-assessed daily-life experiences of CI listeners are an important research area to address. The current study aimed at reviewing the experiences of unilateral CI recipients who do or do not retain a conventional hearing aid in the contralateral ear by a set of questionnaires on bimodal use, hearing disability, hearing handicap and general quality of life. $77 \%$ of bimodal listeners started using a contralateral HA right away after 
receiving the $\mathrm{CI}$, while $50 \%$ of unilateral listeners never tried a contralateral HA. It seemed that not the extent but the quality of the experience with conventional hearing aids prior to receiving the CI differed between both groups. Daily hearing abilities, residual handicap and general quality of life were not significantly different between both groups, which is in line with previous literature. However, when questioning bimodal subjects on the perceived merits of the bimodal combination compared to the CI or HA alone, bimodal benefits were consistently reported across all listening situations. This illustrates that bimodal hearing is not only perceived as valuable in complex situations, but instead can improve listening experiences based on all basic auditory functions. It is therefore important to comprehend what drives a bimodal user to prefer the bimodal combination in comparison to a unilateral subject, even though the resulting overall disability outcome is the same. The demands on daily auditory abilities, personality aspects and the social framework surrounding these subjects therefore seem to ask for further research.

\section{ACKNOWLEDGMENTS}

We thank all respondents for the time and effort taken to fill out the questionnaires. We are grateful for the assistance of S. Otterbeck, M. Truyens and M. van Belle in the translation process of the handicap questionnaire, the help of T.L.C. Scheepers in conducting the data entry check, the contribution of F. Scherf (employed by Advanced Bionics) during the protocol development of this study. Preliminary portions of these data were presented at the 9th Asia Pacific Symposium on Cochlear Implants and Related Sciences (Nov 2013, Hyderabad, India), the 224th Annual Meeting of the Dutch Head and Neck Society (April 2014, Nieuwegein, The Netherlands), the 13th International Conference on Cochlear Implants and Other Implantable Auditory Technologies (June 2014, Munich, Germany), the $8^{\text {th }}$ international Symposium on Objective Measures in Auditory Implants (October 2014, Toronto, Canada), the $18^{\text {th }}$ Jarestagung of the Deutsche Gesellschaft für Audiologie (March 2015, Bochum, Germany) and the Phonak Conference 'Hearing loss and audiological technology between present and future' (June 2016, Milan, Italy).

DISCLOSURE OF INTEREST

A research grant from Advanced Bionics Inc. to MUMC+ financially supported the work of the first author (E.D.) in this investigator-initiated study. The second author (M.J.) reviewed the manuscript and provided statistical support, made possible by a grant from the Dutch Heinsius-Houbolt Foundation. The third author (J.C.) holds a scientific post in the Advanced Bionics European Research Center. For the remaining authors no conflicts were declared. The study was designed in cooperation between MUMC+ and Advanced Bionics. Data collection, analysis and the decision to publish were all solely accounted for by MUMC+. The work presented in this manuscript is the intellectual property of MUMC+. 
APPENDIX 1. Bimodal questionnaire.

Qualitative questionnaire on bimodal hearing aid use and bimodal experiences for subjects who discarded the hearing aid (HA) aside the cochlear implant (CI) (UNI = unilateral group, $\mathrm{n}=22$ ) and subjects who continued to use the HA in the non-implanted ear (BIM = bimodal group, $\mathrm{n}=26$ ). Instructions, questions and results are presented. The origin of each question is described by its reference in literature. Results are presented per group as absolute frequencies as well as percentages across answering alternatives. The overall number of responding subjects per question may differ depending on the completeness of data as well as the relevance of each question in a patient-related manner.

\section{BIMODAL QUESTIONNAIRE}

BIM=bimodal users (continued to use the hearing ead aside the $\mathrm{Cl}$ ) UNI=unilateral users (discarded the hearing aid aside the $\mathrm{Cl}$ )

Instructions: By this questionnaire we hope to gain more insight into the advantages and disadvantages of wearing a hearing aid and the reasons why patients do (not) continue to wear a hearing aid. Please answer each question by checking the box that best represents your situation, experience or vision. Feel free to add any comments in the assigned free space or on the back of the page (please add the number of the question).

Remember the period BEFORE you received a cochlear implant. These questions ask about your experiences with hearing aids during this period.

1. When did you start wearing hearing aids? (Please indicate year and month if possible)

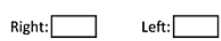

2. When did you stop wearing hearing aids? (Please indicate year and month or "I continued to use a hearing aid untill surgery") Right: $\square$ Left: $\square$

3. How much did you wear your hearing aid(s) before your surgery?

Both sides:

more than 10 hours a day

5 to 10 hours a day less than 5 hours a day

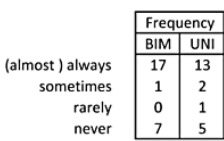

Right: $\quad \begin{array}{r}\text { more than } 10 \text { hours a day } \\ 5 \text { to } 10 \text { hours a day } \\ \text { less than } 5 \text { hours a day }\end{array}$
Left: $\quad$ more than 10 hours a day
5 to 10 hours a day
less than 5 hours a day

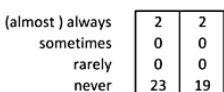

(almost) always \begin{tabular}{ll|}
23 & 19 \\
\hline
\end{tabular} sometrings rarely \begin{tabular}{|c|c|}
\hline 5 & 2 \\
0 & 1 \\
0 & 0 \\
20 & 18 \\
\hline
\end{tabular}

4. Could you hear environmental sounds with your hearing aid(s) before your surgery?

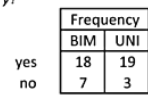

5. Could you understand people talking to you with your hearing aid(s) before your surgery?

With lipreading:

Without lipreading:

6. All in all, how helpful was your hearing aid(s)?

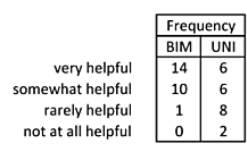

7. How do you feel that you coped with your hearing loss before you received your imlant?

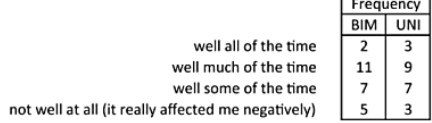

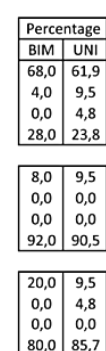
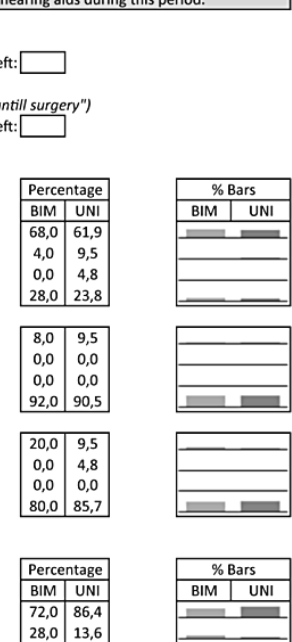
\begin{tabular}{l|l|}
\hline 72,0 & 86,4 \\
28,0 & 13,6 \\
\hline
\end{tabular}

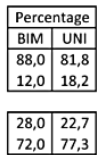

\begin{tabular}{|l|l|}
28,0 & 22,7 \\
72,0 & 77,3 \\
\hline
\end{tabular}

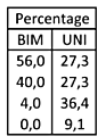

\begin{tabular}{l|l|l|}
28,0 & 31,8 \\
20,0 & 13,6 \\
\hline
\end{tabular}

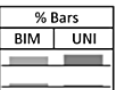

Fitzpatrick et ol. (2010)

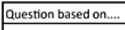

Fitzpatrick et al. (2010)

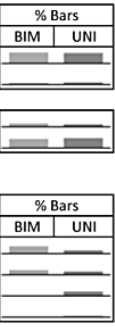

Fitzpatrick et ol. (2010)

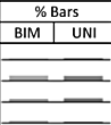


The following questions deal with your decision process about using a hearing aid with the cochlear implant.

8. Had you made a decision before your surgery about whether you would use your hearing aid with your cochlear implant?

\begin{tabular}{c|c|c|}
\multicolumn{1}{c}{} & \multicolumn{2}{c|}{ Frequency } \\
\cline { 2 - 4 } yes & BIM & UNI \\
\cline { 2 - 4 } no & 17 & 7 \\
no & 15 \\
\cline { 2 - 4 }
\end{tabular}$\quad$\begin{tabular}{|c|c|c|}
\multicolumn{2}{|c|}{ Percentage } \\
\hline BIM & UNI \\
\hline 65,4 & 31,8 \\
34,6 & 68,2 \\
\hline
\end{tabular}

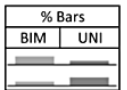

9. Did your audiologist talk to you about the possibility of wearing your hearing aid with your cochlear implant?

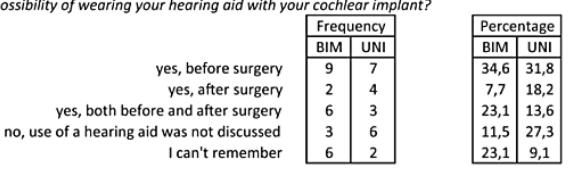

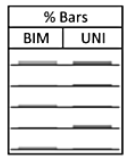

10. Did your audiologist encourage you to try wearing your hearing aid with your cochlear implant?

\begin{tabular}{|c|c|c|c|c|}
\hline & \multicolumn{2}{|c|}{ Frequency } & \multicolumn{2}{|c|}{\begin{tabular}{|l} 
Percentage \\
\end{tabular}} \\
\hline & BIM & UNI & BIM & UNI \\
\hline yes, before surgery & 6 & 1 & 23,1 & 4,5 \\
\hline yes, after surgery & 5 & 4 & 19,2 & 18,2 \\
\hline yes, both before and after surgery & 4 & 4 & 15,4 & 18,2 \\
\hline no & 7 & 9 & 26,9 & 40,9 \\
\hline I can't remember & 4 & 4 & 15,4 & 18,2 \\
\hline
\end{tabular}

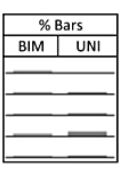

Fitzpatrick et ol. (2010) The following questions deal with your experiences of using a hearing aid AFTER you received the cochlear implant.

11. Has the hearing in your un-implanted ear changed since you received your cochlear implant?

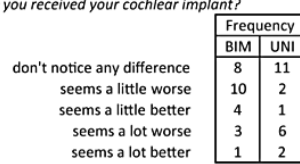

12. How often do you use a hearing aid with your cochlear implant?

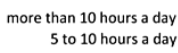

less than 5 hours a day

13. How often do you use only your cochlear implant?

$$
\begin{array}{r}
\text { more than } 10 \text { hours a day } \\
5 \text { to } 10 \text { hours a day } \\
\text { less than } 5 \text { hours a day }
\end{array}
$$

14. How often do you use only a hearing aid?

$$
\begin{array}{r}
\text { more than } 10 \text { hours a day } \\
5 \text { to } 10 \text { hours a day } \\
\text { less than } 5 \text { hours a day }
\end{array}
$$

\begin{tabular}{|c|c|}
\hline \multicolumn{2}{|c|}{ Percentage } \\
\hline BIM & UNI \\
\hline 30,8 & 50,0 \\
38,5 & 9,1 \\
15,4 & 4,5 \\
11,5 & 27,3 \\
3,8 & 9,1 \\
\hline
\end{tabular}

\begin{tabular}{|c|c|}
\hline \multicolumn{2}{|c|}{ Percentage } \\
\hline BIM & UNI \\
\hline 80,8 & 0,0 \\
7,7 & 0,0 \\
11,5 & 0,0 \\
0,0 & 100,0 \\
\hline
\end{tabular}

\begin{tabular}{|c|c|}
\hline \multicolumn{2}{|c|}{ Percentage } \\
\hline BIM & UNI \\
\hline 8,0 & 100,0 \\
8,0 & 0,0 \\
28,0 & 0,0 \\
56,0 & 0,0 \\
\hline
\end{tabular}
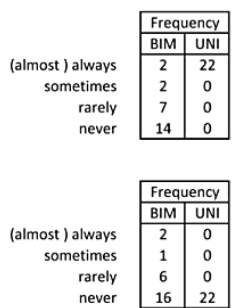

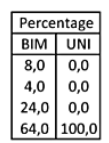

\begin{tabular}{|l|l|}
64,0 & 100,0 \\
\hline
\end{tabular}

\begin{tabular}{|c|c|}
\multicolumn{2}{|c|}{ Percentage } \\
\hline BIM & UNI \\
\hline 76,9 & 27,3 \\
7,7 & 9,1 \\
7,7 & 4,5 \\
7,7 & 9,1 \\
0,0 & 50,0 \\
\hline
\end{tabular}

\begin{tabular}{l|r}
7,7 & 9,1 \\
0,0 & 50,0 \\
\hline
\end{tabular}

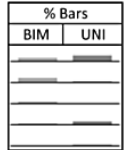

Fitzpatrick et ol. (2009)

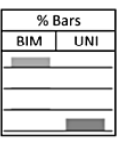

Fitzpatrick et al. (2009)
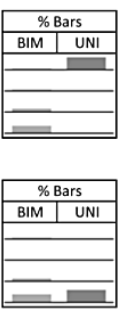

Fitzpatrick et al. (2009)

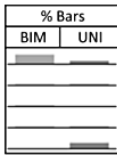

$\rightarrow>$ If you never tried a hearing aid with your cochlear implant: go straight on to question 38.

\begin{tabular}{|c|c|c|}
\multicolumn{1}{c|}{} & \multicolumn{2}{|c|}{ Frequency } \\
\cline { 2 - 3 } & BIM & UNI \\
\cline { 2 - 3 } & 20 & 6 \\
nt & 2 & 2 \\
$n t$ & 2 & 1 \\
nnt & 2 & 2 \\
nn & 0 & 11 \\
\cline { 2 - 3 } & &
\end{tabular}

15. When did you start using your hearing aid and cochlear implant together?

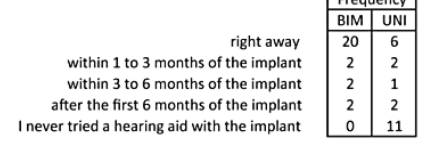


16. After receiving your implant, how long did you use your hearing aid in the other ear?
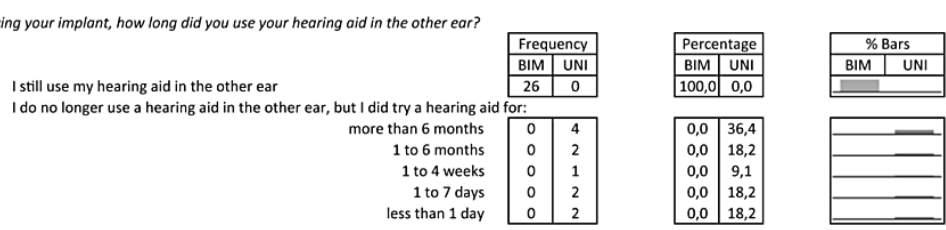

$\rightarrow>$ Please answer the following questions, even if you no longer use a hearing aid aside your implant. You will be asked about your experiences during the period that you did try the hearing aid.

17. How fast did you adapt to using your hearing aid and cochlear implant together?

\begin{tabular}{|c|c|c|c|c|}
\hline & \multicolumn{2}{|c|}{ Frequency } & \multicolumn{2}{|c|}{ Percentage } \\
\hline & BIM & UNI & BIM & \begin{tabular}{|l|l} 
UNI \\
\end{tabular} \\
\hline right away & 18 & 4 & 69,2 & 40,0 \\
\hline within 1 to 3 months of the implant & 4 & 1 & 15,4 & 10,0 \\
\hline within 3 to 6 months of the implant & 2 & 0 & 7,7 & 0,0 \\
\hline after the first 6 months of the imlant & 1 & 0 & 3,8 & 0,0 \\
\hline I couldn't get used to the hearing aid & 1 & 5 & 3,8 & 50,0 \\
\hline
\end{tabular}

18. The hearing aid that you used with your cochlear implant, is this the same hearing aid you used before your surgery?

\begin{tabular}{|c|c|c|c|c|}
\hline & \multicolumn{2}{|c|}{ Frequency } & \multicolumn{2}{|c|}{ Percentage } \\
\hline & BIM & UNI & BIM & UNI \\
\hline yes & 13 & 9 & 50,0 & 90,0 \\
\hline no, I immediately started with a new hearing aid after surgery & 3 & 1 & 11,5 & 10,0 \\
\hline no, first I used the old hearing aid, but now I have a new hearing aid since...... & 10 & 0 & 38,5 & 0,0 \\
\hline
\end{tabular}

19. How old was the hearing aid that you used with your cochlear implant?

\begin{tabular}{r|c|c|}
\cline { 2 - 2 } & \multicolumn{2}{|c|}{ Frequency } \\
\cline { 2 - 3 } more than 10 years old & BIM & UNI \\
\cline { 2 - 3 } 5 to 10 years old & 6 & 1 \\
3 to 5 years old & 10 & 4 \\
less than 3 years old & 10 & 2 \\
\cline { 2 - 3 }
\end{tabular}

\begin{tabular}{|c|c|}
\hline \multicolumn{2}{|c|}{ Percentage } \\
\hline BIM & UNI \\
\hline 0,0 & 11,1 \\
23,1 & 22,2 \\
38,5 & 44,4 \\
38,5 & 22,2 \\
\hline
\end{tabular}

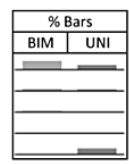

Fitzpatrick et ol. (2009)

40,0

(1) 0,0

\begin{tabular}{|c|c|}
3,8 & 0,0 \\
3,8 & 50,0 \\
\hline
\end{tabular}

Fitzpatrick et al. (2010)

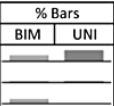

Fitzpatrick et al. (2010)

\begin{tabular}{l|l}
38,5 & 0,0 \\
\hline
\end{tabular}
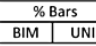

Fitzpatrick et al. (2010)

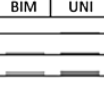

20. Did you need to have your hearing aid adjusted after using your cochlear implant?

not at all, the same settings that were in place before my surgery were used yes, the fitting was changed:

1 to 3 times
3 to 6 times

\begin{tabular}{|c|c|}
\hline \multicolumn{2}{|c|}{ Frequency } \\
\hline BIM & UNI \\
\hline 17 & 6 \\
5 & 1 \\
1 & 2 \\
\hline
\end{tabular}

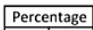

\begin{tabular}{|l|l|}
\hline \multicolumn{2}{|c|}{ Percentage } \\
\hline BIM UNI \\
\hline 65,4
\end{tabular}

\begin{tabular}{|l|l|}
\hline BIM & UNI \\
\hline 65,4 & 66,7 \\
\hline
\end{tabular}

19,2 11,1

\begin{tabular}{c|c|}
19,2 & 11,1 \\
3,8 & 22,2 \\
\hline & 11,5 \\
\hline
\end{tabular}

more than 6 times

\begin{tabular}{c|c|c}
3,8 & 22,2 \\
11,5 & 0,0 \\
\hline
\end{tabular}

These changes were made by:

the audiologist (audiological centre)

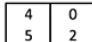

\section{\begin{tabular}{|r|r|}
\hline 44,4 & 0,0 \\
55,6 & 100,0
\end{tabular} \\ \begin{tabular}{|c|c|c|}
55,6 & 100,0 \\
\hline
\end{tabular}} the hearing aid dispenser (shop)

21. How did you set the volume of your hearing aid and cochlear implant when whorn combined? Please check all that apply.

Hearing aid:

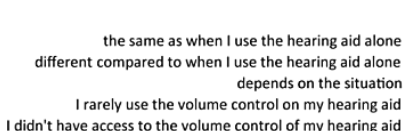
I didn't have access to the volume control of my hearing aid

\begin{tabular}{|c|c|}
\hline \multicolumn{2}{|c|}{ Frequency } \\
\hline BIM & UNI \\
\hline 17 & 3 \\
2 & 2 \\
6 & 2 \\
8 & 3 \\
1 & 1 \\
\hline
\end{tabular}
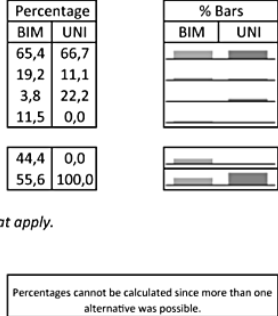

Fitzpotrick et ol. (2009) Fitzpatricket ol. (2010)

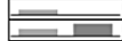

Cochlear implant:

\begin{tabular}{r|c|c|}
\cline { 2 - 2 } the same as when I use the cochlear implant alone & 17 & 5 \\
different compared to when I use the cochlear implant alone & 0 & 1 \\
depends on the situation & 7 & 2 \\
I rarely use the volume control on my cochlear implant & 7 & 1 \\
I didn't have access to the volume control of my cochlear implant & 1 & 1 \\
\hline
\end{tabular}

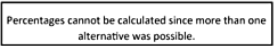

I didn't have access to the volume control of my cochlear implant

22. How did you adjust the program of your hearing aid and the cochlear implant when whorn together? Please check all that apply.

Hearing aid:

the same as when I use the hearing aid alone
different compared to when I use the hearing aid alone
depends on the situation

\begin{tabular}{|c|c|}
\hline \multicolumn{2}{|c|}{ Frequency } \\
\hline BIM & UNI \\
\hline 18 & 5 \\
0 & 0 \\
5 & 2 \\
5 & 2 \\
2 & 1 \\
\hline
\end{tabular}

Cochlear implant:

$$
\begin{array}{r|c|c|}
\text { the same as when I use the cochlear implant alone } & 15 & 7 \\
\text { different compared to when I use the cochlear implant alone } & 1 & 1 \\
\text { depends on the situation } & 10 & 3 \\
\text { I rarely use different programs on my cochlear implant } & 6 & 0 \\
\text { I don't have access to the program function of my cochlear implant } & 1 & 0 \\
\cline { 2 - 4 }
\end{array}
$$

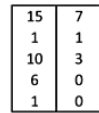


23. In which of the following settings did you wear your hearing aid with your cochlear implant? Please check all that apply.

$$
\begin{array}{c|c|c|}
\cline { 3 - 3 } & \multicolumn{2}{|c|}{\text { Frequency }} \\
\cline { 2 - 3 } \text { I use(d) my hearing aid at work } & 1 & \text { UNI } \\
\cline { 3 - 3 } \text { I use(d) my hearing aid at home } & 3 & 5 \\
\text { I use(d) my hearing aid everywhere I went } & 23 & 5 \\
\cline { 3 - 3 } & &
\end{array}
$$

24. In wich of the following listening conditions did you wear your hearing aid with your cochlear implant? Please check all that apply.

$$
\begin{array}{r|c|c|}
\cline { 2 - 3 } & \multicolumn{2}{|c}{\text { Frequency }} \\
\cline { 2 - 3 } \text { I use(d) my hearing aid in noisy situations } & 22 & 4 \\
\text { I use(d) my hearing aid in quiet situations } & 25 & 9 \\
\text { I use(d) my hearing aid when listening to music } & 22 & 5 \\
\text { other listening situations: .... } & 0 & 0 \\
\hline
\end{array}
$$

\begin{tabular}{|c|c|c|c|c|}
\hline \multirow{2}{*}{ 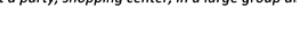 } & \multicolumn{2}{|c|}{ Frequency } & \multicolumn{2}{|c|}{\begin{tabular}{|l} 
Percentage \\
\end{tabular}} \\
\hline & BIM & UNI & BIM & \begin{tabular}{|l|l} 
UNI \\
\end{tabular} \\
\hline cochlear implant only & 0 & 5 & 0,0 & 55,6 \\
\hline hearing aid only & 2 & 0 & 7,7 & 0,0 \\
\hline hearing aid together & 20 & 2 & 76,9 & 22,2 \\
\hline no difference with any of the above & 4 & 2 & 15,4 & 22,2 \\
\hline
\end{tabular}

\begin{tabular}{|c|c|}
\hline \multicolumn{2}{|c|}{ Percentage } \\
\hline BIM & UNI \\
\hline 0,0 & 60,0 \\
\hline 0,0 & 0,0 \\
\hline 100,0 & 0,0 \\
\hline 0,0 & 40,0 \\
\hline ith you & \\
\hline \begin{tabular}{|l|l|} 
Perce \\
\end{tabular} & ntage \\
\hline BIM & UNI \\
\hline 0,0 & 55,6 \\
\hline 7,7 & 0,0 \\
\hline 76,9 & 22,2 \\
\hline 15,4 & 22,2 \\
\hline
\end{tabular}

25. In quiet listening situations (e.g. a 1-on-1 conversation), you hear better with your:

$$
\begin{array}{rl|c|} 
& \multicolumn{2}{|c|}{\text { Frequency }} \\
\cline { 2 - 3 } \text { cochlear implant only } & \text { BIM } & \text { UNI } \\
\cline { 2 - 3 } \text { hearing aid only } & 0 & 6 \\
& 0 & 0 \\
\text { cochlear implant and hearing aid together } & 25 & 0 \\
\text { no difference with any of the above } & 0 & 4 \\
\cline { 3 - 3 } &
\end{array}
$$

\begin{tabular}{l|l|l|l}
25 & 9 \\
22 & 5 \\
\hline
\end{tabular}

26. In noisy situations (e.g. in a restaurant, at a party, shopping center, in a large group discussion), you hear better with your:

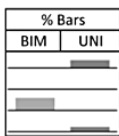

Fitzpatrick et al. (2009) no difference with any of the above \begin{tabular}{c|c}
20 & 2 \\
4 & 2
\end{tabular}

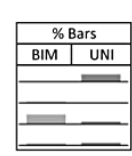

27. In places with an echo (e.g. church, auditorium), you hear better with your:

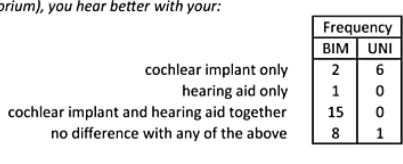

$$
\begin{array}{r}
\text { cochlear implant only } \\
\text { hearing aid only }
\end{array}
$$

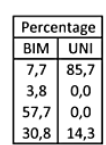

\begin{tabular}{r|r|r|}
57,7 & 0,0 \\
30,8 & 14,3 \\
\hline
\end{tabular}
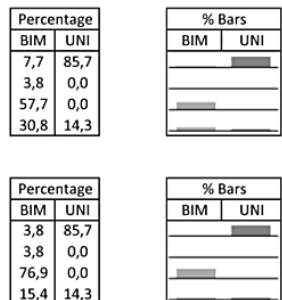

\begin{tabular}{|l|l|l|}
\hline 15,4 & 14,3 \\
\hline
\end{tabular}

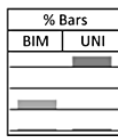

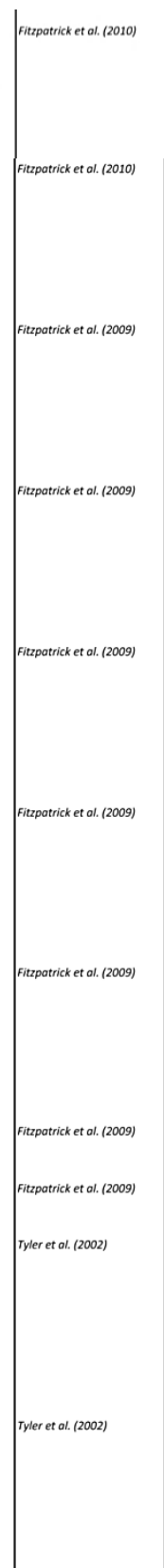

29. Telling from which direction souds are coming is easier with your:

$$
\begin{array}{r}
\text { cochlear implant only } \\
\text { hearing aid only } \\
\text { cochlear implant and hearing aid together } \\
\text { no difference with any of the above }
\end{array}
$$

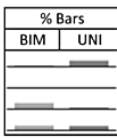

30. Are there certain sounds that you hear better (or worse) when wearing your hearing aid and cochlear implant together? Describe which sounds, e.g. sounds in the enfironment (e.g. telephone, water), speech sounds (e.g. consonants, vowels, quiet voices, etc.)

31. Please use the space below and the reverse side of this page to share any additional information about how you feel that using a hearing aid and cochlear implant together helps or hinders listening and communication.

32. When you are using your hearing aid and cochlear implant together, where do you perceive sound?

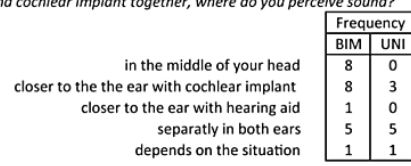

\begin{tabular}{|c|c|}
\hline \multicolumn{2}{|c|}{ Percentage } \\
\hline BIM & UNI \\
\hline 34,8 & 0,0 \\
34,8 & 33,3 \\
4,3 & 0,0 \\
21,7 & 55,6 \\
4,3 & 11,1 \\
\hline
\end{tabular}

33. When you are using your hearing aid and cochlear implant together, where is the sound louder?

\begin{tabular}{c|c|c|} 
& \multicolumn{2}{|c|}{ Frequency } \\
\cline { 2 - 3 } in the cochlear implant ear & BIM & UNI \\
\cline { 2 - 3 } in the hearing aid ear & 1 & 7 \\
same loudness in both ears & 7 & 1 \\
depends on the situation & 0 & 0 \\
\cline { 2 - 3 } & &
\end{tabular}
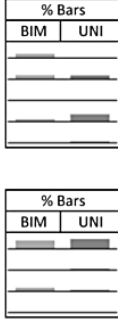

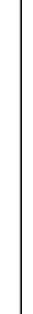


34. When using the cochlear implant and hearing aid together, does the sound "fuse" or "join" to become one image?

\begin{tabular}{c|c|c|}
\cline { 2 - 3 } & \multicolumn{2}{|c|}{ Frequency } \\
\cline { 2 - 3 } & BIM & UNI \\
\cline { 2 - 3 } yes, it fuses together to become one image & 16 & 1 \\
no, I hear two separate images & 5 & 7 \\
different, namely: ..... & 3 & 1 \\
\cline { 2 - 3 } & &
\end{tabular}

\begin{tabular}{|l|l|}
\hline \multicolumn{2}{|c|}{ Percentage } \\
\hline BIM & UNI \\
\hline 66,7 & 11,1 \\
20,8 & 77,8 \\
12,5 & 11,1 \\
\hline
\end{tabular}

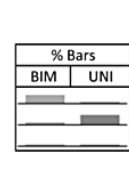

Iyler et al. (2002)

35. Which ear contributes most to understanding speech

$$
\begin{array}{r}
\text { especially the cochlear implant ear } \\
\text { rather the cochlear implant ear } \\
\text { the hearing aid and cochlear implant ear add equally } \\
\text { rather the hearing aid ear }
\end{array}
$$

\begin{tabular}{|c|c|}
\hline \multicolumn{2}{|c|}{ Frequency } \\
\hline BIM & UNI \\
\hline 12 & 10 \\
2 & 0 \\
9 & 0 \\
1 & 0 \\
2 & 0 \\
\hline
\end{tabular}

\begin{tabular}{|c|c|}
\hline \multicolumn{2}{|c|}{ Percentage } \\
\hline BIM & UNI \\
\hline 46,2 & 100,0 \\
7,7 & 0,0 \\
34,6 & 0,0 \\
3,8 & 0,0 \\
7,7 & 0,0 \\
\hline
\end{tabular}

36. Considering everything, do you think your hearing aside the cochlear implant is worth the trouble?

\begin{tabular}{c|c|c|} 
& \multicolumn{3}{c|}{} \\
\cline { 2 - 3 } no, not worthwhile at all & 1 & 7 \\
a bit worthwhile & 2 & 1 \\
\cline { 2 - 3 } reasonably worthwhile & 4 & 0 \\
really worthwhile & 8 & 1 \\
extremely worthwhile & 11 & 0 \\
\cline { 2 - 3 } & &
\end{tabular}

\begin{tabular}{|c|c|}
\hline \multicolumn{2}{|c|}{ Percentage } \\
\hline BIM & UNI \\
\hline 3,8 & 77,8 \\
7,7 & 11,1 \\
15,4 & 0,0 \\
30,8 & 11,1 \\
42,3 & 0,0 \\
\hline
\end{tabular}
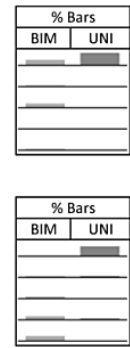

37. Considering everything, how much has your hearing aid aside the cochlear implant changed your enjoyment in life?

\begin{tabular}{r|c|c|}
\multirow{1}{*}{ worse } & 0 & 3 \\
\cline { 2 - 3 } no difference & 2 & 5 \\
a bit better & 5 & 0 \\
better & 7 & 0 \\
much better & 12 & 1 \\
\cline { 2 - 3 } & &
\end{tabular}

\begin{tabular}{|c|c|}
\hline \multicolumn{2}{|c|}{ Percentage } \\
\hline BIM & UNI \\
\hline 0,0 & 33,3 \\
7,7 & 55,6 \\
19,2 & 0,0 \\
26,9 & 0,0 \\
46,2 & 11,1 \\
\hline
\end{tabular}

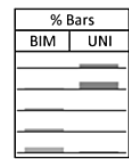

38. List the 3 factors that most influenced your decision (not) to use year hearing aid with the cochlear implant (from 1 to 3 in order of importance).

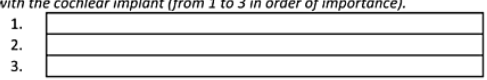

39. Please feel free to share any additional information concerning the reasons why you decided (not) to wear your hearing aid in conjunction with your

\section{Bibliography}

Cox, R. M., Stephens, D., \& Kramer, S. E. (2002). Translations of the International Outcome inventory for Hearing Aids (IOI-HA) Jnternational Journal of Audiology, 41 (1), 3-26. Fitzpatrick, E. M., \& Leblanc, S. (2010). Exploring the factors influencing discontinued hearing aid use in patients with unilateral cochlear implants Trends in Amplification , 14 (4), $199-210$. Fitzpatrick, E. M., Seguin, C., Schramm, D., Chenier, J., \& Armstrong, S. (2009). Users' experience of a cochlear implant combined with a hearing aidJ/nt J Audiol, 48 (4), $172-182$. Tyler, R. S., Parkinson, A. J., Wilson, B. S., Witt, S., Preece, J. P., \& Noble, W. (2002). Patients utilizing a hearing aid and a cochlear implant: speech perception and localizationEar and Hearing , 23 (2), 98-105. 
APPENDIX 2. Pragmatic subscales SSQ.

Hearing (dis)ability ratings for subjects who only use a cochlear implant (CI) (unilateral group, $n=20$ ) and subjects who also use a hearing aid (HA) in the non-implanted ear (bimodal group, $\mathrm{n}=24$ ). Pooled mean (+standard error) scores for the 10 pragmatic subscales on a visual-analogue scale (VAS, 0-10) using the SSQ-questionnaire by Gatehouse \& Noble (2004). Scores were compared between groups as fitted (A.) and evaluated within the bimodal group for listening conditions with $\mathrm{CI}$, with $\mathrm{HA}$ and with $\mathrm{CI}$ and $\mathrm{HA}$ together (B.) Asterisks denote significant differences between groups or listening conditions ${ }^{*} p<0.05$, ** $\left.\mathrm{p}<0.01,{ }^{* * *} \mathrm{p}<0.001\right)$.

\section{A. Between groups (as fitted)}

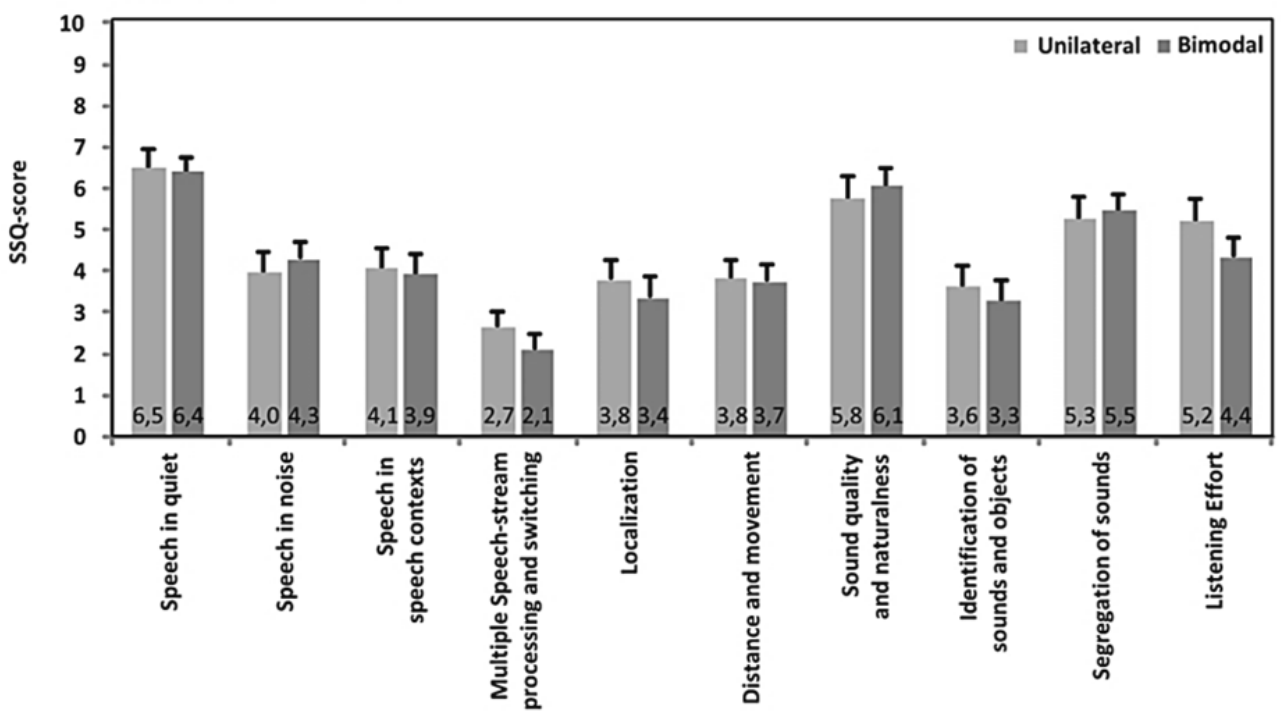




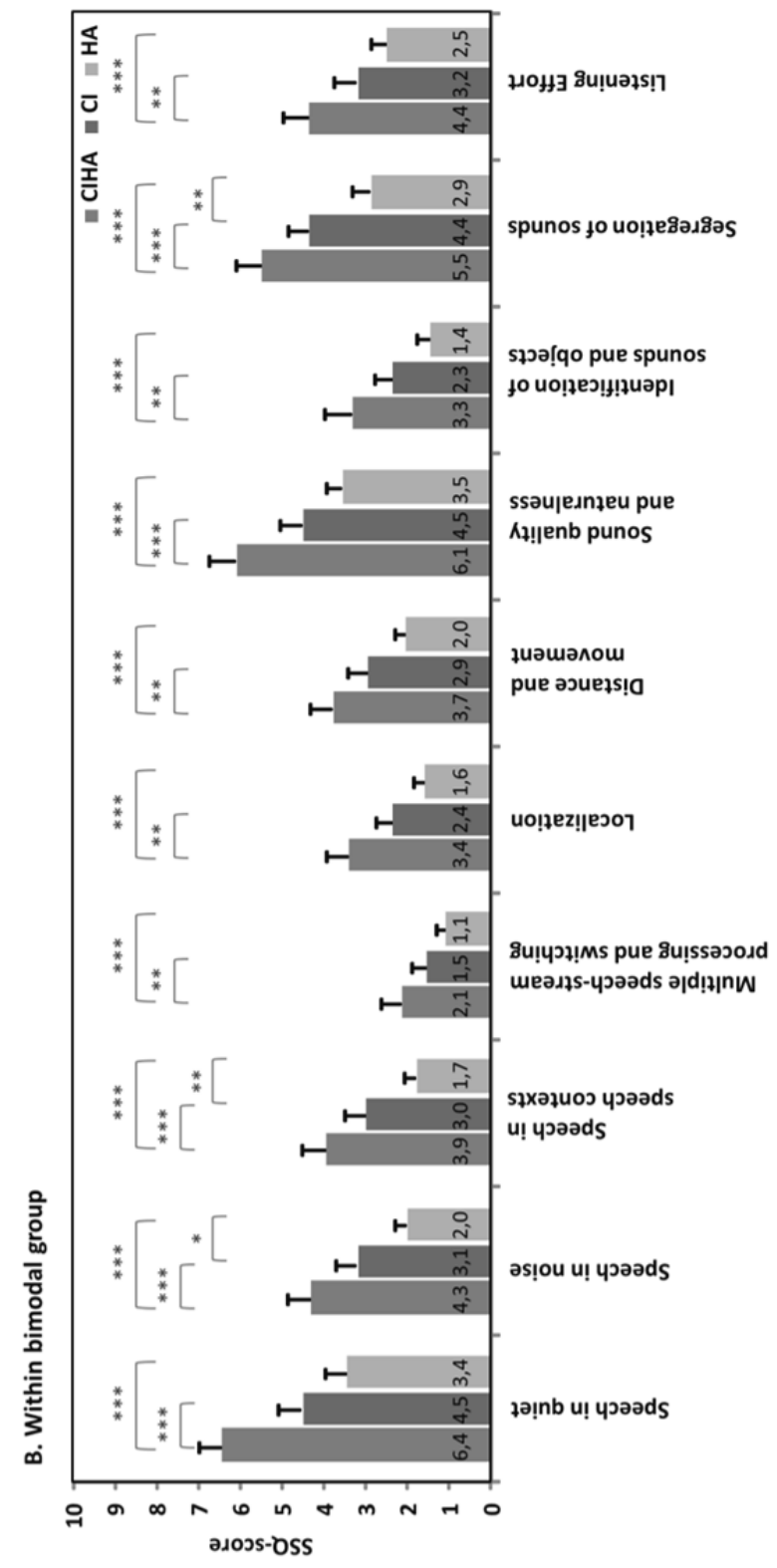





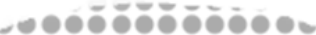

-

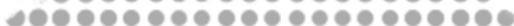

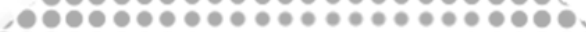

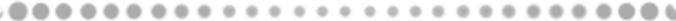

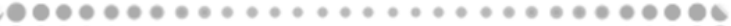

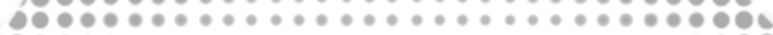



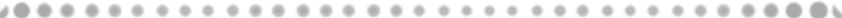

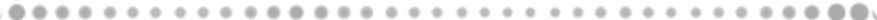

เ

분

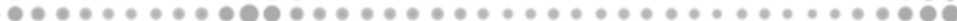

เ

,

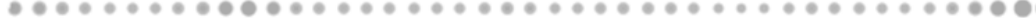

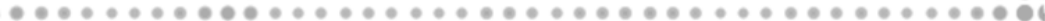

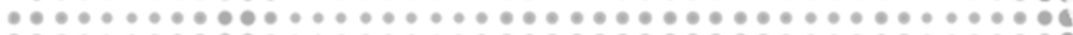

ㄴ․

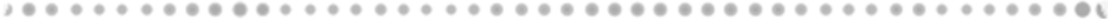

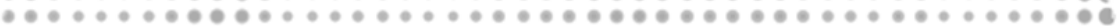

\section{c}

THE BENEFITS OF BIMODALAIDING ON EXTENDED DIMENSIONS

OF SPEECH PERCEPTION: INTELLIGIBILITY LISTENING EFFORT,

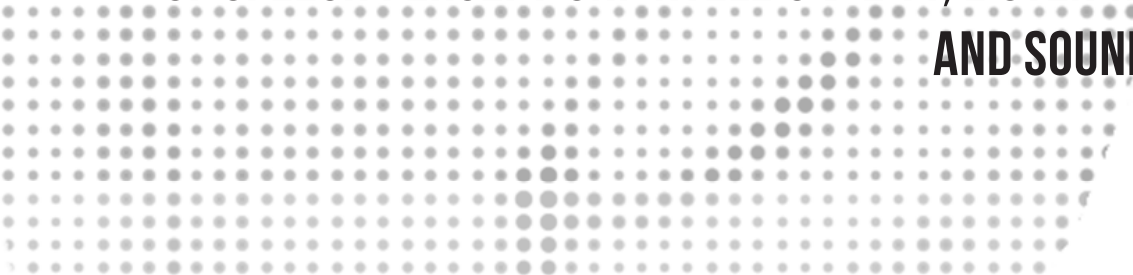

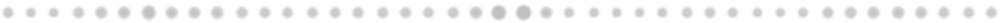

a.

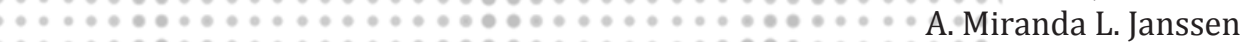

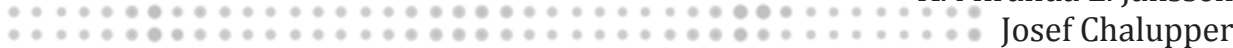

$40 \%$ Robert J. Stokroos

Erwin L.J. George

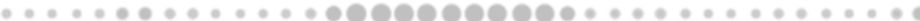

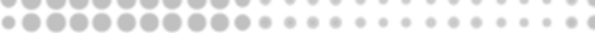

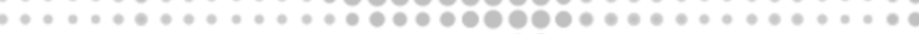

…

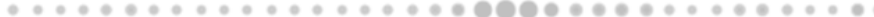

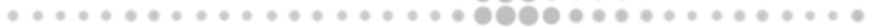

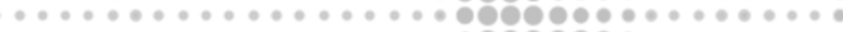

OPिPि?

Qि口िि

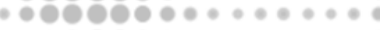

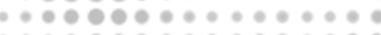

.

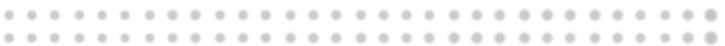

* +

Trends in Hearing, 2017, Volume 21:1-20

DOI: $10.1177 / 2331216517727900$ 
The benefits of combining a cochlear implant (CI) and a hearing aid (HA) in opposite ears on speech perception were examined in 15 adult unilateral CI recipients who regularly use a contralateral HA. A withinsubjects design was carried out to assess speech intelligibility testing, listening effort ratings, and a sound quality questionnaire for the conditions $\mathrm{CI}$ alone, CIHA together, and HA alone when applicable. The primary outcome of bimodal benefit, defined as the difference between CIHA and CI, was statistically significant for speech intelligibility in quiet as well as for intelligibility in noise across tested spatial conditions. A reduction in effort on top of intelligibility at the highest tested signal-tonoise ratio was found. Moreover, the bimodal listening situation was rated to sound more voluminous, less tinny, and less unpleasant than $\mathrm{CI}$ alone. Listening effort and sound quality emerged as feasible and relevant measures to demonstrate bimodal benefit across a clinically representative range of bimodal users. These extended dimensions of speech perception can shed more light on the array of benefits provided by complementing a CI with a contralateral HA. 


\section{INTRODUCTION}

\section{BIMODAL AIDING}

As inclusion criteria for receiving a cochlear implant (CI) have been expanded to include candidates with hearing loss ranging from severe to moderate, a trend has been observed toward more residual hearing in the non-implanted ear (Gifford et al, 2010; Hughes et al, 2014). More than $60 \%$ of a recent sample of unilateral CI recipients with aidable residual hearing opted to retain a conventional hearing aid (HA) in the contralateral ear (Devocht et al, 2015). The bimodal combination of a $\mathrm{CI}$ and a HA (CIHA) has the potential of providing access to bilateral, binaural, and complementary cues to overcome some shortcomings in unilateral CI performance (Ching et al, 2007; Olson \& Shinn, 2008; Schafer et al, 2011). In this growing population of bimodal users, it is important to have a set of practicable outcome measures to enable evaluation of the bimodal benefits of speech perception across the full clinical range of aided recipients to optimize hearing performance. The goal for fitting a HA should be to optimize speech understanding while maximizing listening comfort (Hällgren et al, 2005) and doing so in a qualitatively acceptable way. This calls for an exploration of the dimensions of intelligibility, listening effort, and sound quality within this context.

\section{SPEECH INTELLIGIBILITY}

In the past, clinicians have often not extended testing beyond speech intelligibility in quiet conditions. However, increasingly higher levels of CI performance suggest that testing in quiet does not sufficiently cover the difficulty range to document performance in current CI populations (Ebrahimi-Madiseh et al, 2016; Gifford et al, 2008). Beyond the fact that testing in noise better reflects real-life situations than testing in quiet, it has also been shown that speech intelligibility in noise is a better metric for evaluating the maximum potential of bimodal aiding (Dorman et al, 2015). In particular, a set-up with spatially separated speech and noise sources is known to demonstrate the extent to which participants can profit in intelligibility from binaural aiding (Avan et al, 2015).

When testing intelligibility in noise (Schafer et al, 2011), the speech reception threshold (SRT), commonly defined as the signal-to-noise ratio (SNR) at which the listener is able to understand $50 \%$ of the signal correctly, can be elicited using an adaptive paradigm (Treutwein, 1995). The advantage of such adaptive procedures is that they are not susceptible to floor or ceiling effects. At the same time, testing in noise with sentences at threshold levels is a difficult task, in particular for CI listeners (Kaandorp et al, 2015; Schafer et al, 2011). This can increase uncertainty 
regarding outcomes, resulting in a higher SRT than the actual SRT (Smits \& Festen, 2011) and high test-retest differences (Kaandorp et al, 2015). Kaandorp et al. (2015) recently proposed setting the upper limit for reliable SRT outcomes at $+15 \mathrm{~dB}$ SNR. Higher levels, namely, suggest that the adaptive procedure has not resulted in a reliable qualification of speech perception according to the speechintelligibility-index model in stationary noise (ANSI S3.5, 1997). More commonly however a selection bias is applied to avoid these difficulties by establishing a minimum required level of performance in quiet (e.g., 50\%) before testing in noise (e.g. Buechner et al. 2014; Cullington \& Zeng 2011; Nelson \& Jin 2004). Individual CI scores can range from 0 up to $100 \%$ within representative bimodal populations (Devocht et al, 2015; Dorman et al, 2015; Dorman et al, 2008; Zhang et al, 2013). Information regarding intelligibility testing in noise is then often unreliable or not available for a subgroup of bimodal subjects. The current study included bimodal subjects across the clinical variety of performance outcomes. Selection criteria for testing intelligibility in noise were not established while documentation was made of subjects who failed to achieve a reliable SRT outcome. Other measures beyond intelligibility were examined to determine their ability to evaluate bimodal speech perception benefits across the total range of bimodal users.

\section{Extended Dimensions of Speech Perception}

Speech perception is multidimensional by nature (Gatehouse \& Noble, 2004; Grancharov et al, 2007; Preminger \& Van Tasell, 1995a; Sockalingam et al, 2009). Commonly distinguished dimensions are as follows: intelligibility or performance, pleasantness or naturalness or satisfaction, loudness, and listening effort or ease. Evaluating HA outcomes should therefore include dimensions beyond aided speech intelligibility, such as sound quality, listening effort, subjective benefit, satisfaction, or use (Humes, 1999). Intelligibility is not only the most commonly tested dimension, but it is also the most dominant one since all other dimensions correlate strongly with the level of intelligibility when intelligibility is allowed to vary over a wide range (Preminger \& Van Tasell, 1995b). Other dimensions of speech perception can therefore only be observed as being unique in themselves once the level of intelligibility has been stabilized (Preminger \& Van Tasell, 1995a).

\section{LISTENING EFFORT}

Listening effort is often loosely defined (Chang et al, 2016) but generally refers to the attention and cognitive requirements of speech perception, especially in adverse listening situations (Rönnberg et al, 2014; Rudner et al, 2012). listening effort can improve even beyond the level where intelligibility has reached its maximum (Klink et al, 2012a). Approaches to assess listening effort include subjective, behavioral, or physiological methods (Ohlenforst et al, 2017). It has 
been suggested that alternative or varying procedures tap into different underlying mechanisms of listening effort (Rudner et al, 2012). A common conceptual framework for listening effort across studies is however lacking (Ohlenforst et al, 2017). When looking for a method that can be easily and quickly applied in a clinical setting (van Esch et al, 2013; Rudner et al, 2012)but at the same time is sensitive to differences between listening conditions (Brons et al, 2013; Hällgren et al, 2005; Humes, 1999; Rudner et al, 2012; Zekveld et al, 2010), a subjective procedure is often the obvious choice. It is known that binaural listening requires less listening effort than monaural hearing (Feuerstein, 1992). Reduced effort by bimodal hearing has been observed in the form of shorter response times (Chang et al, 2016; Luo et al, 2014). Questionnaire ratings of overall daily effort, however, could not establish a significant difference between subgroups of bimodally aided CI recipients and other groups of CI users (Farinetti et al, 2015; Goman, 2014; Noble et al, 2008b). In the current study, a subjective rating procedure was used in an attempt to demonstrate bimodal listening effort benefit as a supplemental dimension of intelligibility.

\section{SOUND QUALITY}

Strictly speaking, sound quality refers to the naturalness of sound, as denoted by its color, timbre, or character (Slawson, 1985). Although a subjective assessment of sound quality is considered valuable and clinically feasible, a generally accepted clinical protocol is lacking (Eisenberg et al, 1997; Sockalingam et al, 2009). Many studies have assessed sound quality in a one-dimensional way by looking upon it as a preference measurement (e.g. Koning et al. 2015; Suelzle et al. 2013)or a broad component in an overall questionnaire (Amann \& Anderson, 2014; Gatehouse \& Noble, 2004). Nevertheless it has been widely accepted that sound quality is a multidimensional phenomenon (Preminger \& Van Tasell, 1995b). When describing characteristics of hearing instruments, multiple perceptual attributes have been used, such as sharpness, clearness, darkness, fullness, nearness, loudness, and smoothness (Balfour \& Hawkins, 1992; Boretzki, 1999; Gabrielsson, 1979). It is noted that binaural compared with monaural hearing gives rise to substantial differences in overall impression, fullness, and spaciousness (Balfour \& Hawkins, 1992). Hearing electrically by a CI or acoustically by a HA is also known to produce different sound qualities (McDermott \& Sucher, 2006). Bimodal listeners report that the CI alone sounds artificial and alien (Crew et al, 2015), while supplementing with the HA bimodally makes sounds more natural (Armstrong \& Pegg, 1997; Crew et al, 2015; Most et al, 2012), more speech-like (Hamzavi et al, 2004), richer, and more colorful (Zhang et al, 2013). An improvement in sound quality is often a reported reason for CI users to retain a contralateral HA for bimodal listening (Ching et al, 2007; Flynn \& Schmidtke, 2004; 
Scherf \& Arnold, 2014; Sucher \& McDermott, 2009). The current study aimed to investigate the qualitative benefits of bimodally combining electric and acoustic inputs in opposite ears while considering sound quality in a multidimensional way.

\section{CURRENT Study}

This study aimed at assembling a test battery of speech perception to evaluate the benefits of combining a $\mathrm{CI}$ and a HA in opposite ears across a representative group of bimodally aided recipients. Monosyllabic intelligibility scores in quiet were considered as a basic reference. The outcomes and applicability of a spatial speech intelligibility in noise test were evaluated. It was expected that listening bimodally with CI and HA together would improve the SRT within a spatial set-up as compared with listening with $\mathrm{CI}$ alone. However, it was anticipated that not all subjects would be able to achieve reliable SRT outcomes when testing intelligibility in noise. It was hypothesized that extended dimensions of speech perception, namely listening effort and multiple attributes of sound quality, would provide extra insights into bimodal aiding and have the benefit of being applicable to a wide range of $\mathrm{CI}$ users. By testing at fixed levels of intelligibility, it was expected that listening effort and sound quality could be addressed as being unique dimensions in themselves rather than being related to intelligibility. It was hypothesized that listening bimodally would reduce listening effort as compared with listening with CI alone especially at levels where intelligibility already had reached a maximum. Sound quality ratings were also expected to change when adding the $\mathrm{HA}$ aside the $\mathrm{CI}$, particularly for those attributes related to the addition of low-frequency acoustic hearing. The relationship between the observed bimodal benefits and the amount of residual hearing was also examined.

\section{Materials and MethodS}

\section{ETHICS}

The local Medical Ethical Committee (Maastricht University Medical Center, NL42011.068.13) approved this study as part of a larger clinical trial registered in the Dutch National Trial Register (NTR3932) and conducted in accordance with the Declaration of Helsinki. All participants provided written informed consent prior to participation, received a modest participation fee, and were compensated for their travelling expenses.

\section{Participants}

Fifteen bimodal adult patients of the CI team South-East Netherlands were enrolled in this study (eight male and seven female, mean age: 62.0 years, $S D$ : 
12.5 years). All participants were Dutch speaking, had at least 1 year of regular experience with a CI of the brand Advanced Bionics ${ }^{\mathrm{TM}}$ (Valencia, United States) and self-reported that they consistently used a conventional HA in the contralateral ear for at least $50 \%$ of the day. Details on the participants' individual hearing situation are presented in Table 1. Unaided and aided hearing thresholds in the HA-ear are plotted in Figure 1. Participants were found to have considerable residual hearing in the HA-ear with acoustically aidable thresholds of up to $1000 \mathrm{~Hz}$ on average. The mean pure-tone average (PTA across 250, 500, and $1000 \mathrm{~Hz}$ ) was found to be 81.6 dB HL (SD: $18.3 \mathrm{~dB}$ ) in the unaided and $36.0 \mathrm{~dB}$ HL $(S D: 7.4 \mathrm{~dB})$ in the aided situation.

TABLE 1. Subject characteristics

\begin{tabular}{|c|c|c|c|c|c|c|c|c|}
\hline \multirow[b]{2}{*}{$\frac{\text { ¿ू }}{\frac{d}{3}}$} & \multirow[b]{2}{*}{ 잉 } & \multicolumn{4}{|c|}{ CI } & \multicolumn{3}{|c|}{ HA } \\
\hline & & 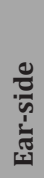 & 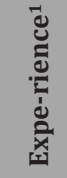 & 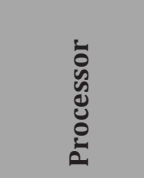 & क्ठ & 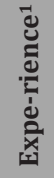 & 를 & $\sum_{0}^{\infty}$ \\
\hline B03 & Meningitis & $\mathrm{R}$ & 4,4 & Harmony ${ }^{2}$ & HiRes-S & 55 & Oticon ${ }^{3}$ & Swift $120+$ \\
\hline B06 & Otosclerosis & $\mathrm{R}$ & 5,0 & Harmony ${ }^{2}$ & HiRes-S/Fid120 & 26 & Siemens ${ }^{4}$ & Nitro $701 \mathrm{SP}$ \\
\hline B08 & Unknown & $\mathrm{R}$ & 7,1 & Harmony ${ }^{2}$ & HiRes-P/Fid 120 & 51 & Oticon ${ }^{3}$ & Ino Pro P \\
\hline $\mathrm{B} 10$ & Unknown & $\mathrm{L}$ & 6,0 & $\begin{array}{l}\text { Naida CI } \\
\text { Q70² }\end{array}$ & HiRes Optima-S & 26 & Phonak $^{5}$ & Naida IX UP \\
\hline B12 & Unknown & $\mathrm{R}$ & 3,2 & Harmony ${ }^{2}$ & HiRes-S/Fid120 & 27 & Oticon $^{3}$ & Sumo DM \\
\hline B15 & $\begin{array}{l}\text { Hereditary; } \\
\text { Trauma }\end{array}$ & $\mathrm{R}$ & 4,3 & Harmony ${ }^{2}$ & HiRes-S/Fid 120 & 19 & Oticon ${ }^{3}$ & Agil \\
\hline B20 & Rubella & $\mathrm{L}$ & 4,8 & Harmony ${ }^{2}$ & HiRes-S/Fid 120 & 52 & Oticon ${ }^{3}$ & Sumo XP \\
\hline B22 & Noise & $\mathrm{R}$ & 1,8 & Harmony $^{2}$ & HiRes-P/Fid 120 & 17 & Phonak $^{5}$ & Naida S I UP \\
\hline B26 & $\begin{array}{l}\text { Turner } \\
\text { syndrome }\end{array}$ & $\mathrm{R}$ & 2,3 & Harmony $^{2}$ & HiRes-S/Fid 120 & 47 & Oticon ${ }^{3}$ & $380 \mathrm{P}$ \\
\hline B34 & $\begin{array}{l}\text { Viral } \\
\text { infection }\end{array}$ & $\mathrm{L}$ & 3,8 & Harmony ${ }^{2}$ & HiRes-S & 29 & Phonak $^{5}$ & Naida V UP \\
\hline B37 & Meningitis & $\mathrm{R}$ & 6,8 & Harmony $^{2}$ & HiRes-S/Fid 120 & 45 & Phonak $^{5}$ & Naida S III UP \\
\hline B42 & $\begin{array}{l}\text { Cogan } \\
\text { syndrome }\end{array}$ & $\mathrm{R}$ & 6,5 & Harmony $^{2}$ & HiRes-S & 11 & Phonak $^{5}$ & Naida V UP \\
\hline B43 & Meningitis & $\mathrm{L}$ & 4,8 & Harmony $^{2}$ & HiRes-S/Fid120 & 61 & Phonak $^{5}$ & Naida IX UP \\
\hline B45 & Meningitis & $\mathrm{R}$ & 1,9 & Neptune $^{2}$ & HiRes-S & 42 & Phonak $^{5}$ & Naida S IX UP \\
\hline B47 & $\begin{array}{l}\text { Hereditary; } \\
\text { Meniere }\end{array}$ & $\mathrm{R}$ & 1,3 & Harmony² & HiRes-S/Fid120 & 28 & Phonak $^{5}$ & Naida V UP \\
\hline
\end{tabular}


FIGURE 1. Residual hearing.
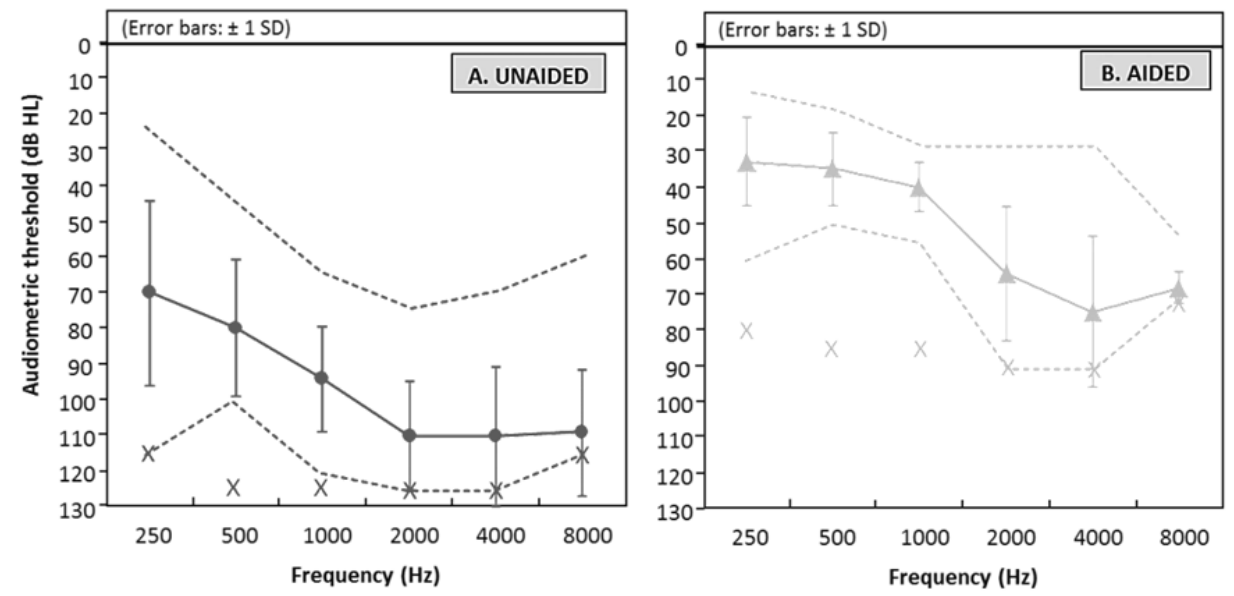

Mean pure-tone air conduction thresholds in the hearing aid ear for the unaided situation under headphones (a) and the aided situation in free field (b). Dashed lines denote the range of observed thresholds. If no response could be recorded within the limits of the audiometer, a value of $5 \mathrm{~dB}$ HL greater than the maximum tested level was entered (see X markings).

\section{ProceDURES}

A within-subjects design was used to assess the performance of bimodal CI recipients on different dimensions of speech perception: speech intelligibility, listening effort, and sound quality.

Measurements were performed in a sound-attenuated booth during one acute test session. The order of tests was fixed across participants. The spatial speech-innoise test was performed first. Then the sound quality scales were completed, followed by listening effort rating. To counteract fatigue, breaks were taken between and during tests when necessary.

All participants used their own hearing devices at typical daily use settings and manipulations during the course of testing were not allowed. Both the CI speech processor and the HA were checked to ensure they were working correctly. There were two participants whose CI speech processor was different from the others (see Table 1). For these participants, the daily CI mapping was adopted into a Harmony ${ }^{\mathrm{TM}}$ speech processor with $\mathrm{T}-\mathrm{mic}^{\mathrm{TM}}$ (Frohne-Büchner et al, 2004) to achieve consistency across participants. When testing a monaural condition, the 
contralateral device was turned off and left in situ. Since the CI-ear is assumed to be the primary speech input for most of the CI-recipients (Neuman et al, 2017), the primary outcome of bimodal benefit was defined as the benefit of listening with CI and HA together compared with the reference of listening with CI alone. As a consequence and by taking test and time constraints into account, the outcomes of the spatial speech-in-noise test and listening effort rating were not measured for the HA alone condition.

\section{SPEECH INTELLIGIBILITY IN QUIET}

Word intelligibility was retrieved from the last standard clinical routine measurement (less than 12 months prior to the acute test session). The maximum phoneme score (\%) over the levels 55,65 , and $75 \mathrm{~dB}$ SPL on a Dutch monosyllabic consonant- nucleus-consonant (CNC) intelligibility test (Bosman \& Smoorenburg, 1995)was recorded in quiet from the frontal direction for the listening condition with $\mathrm{CI}$, with HA, and with CIHA together.

\section{SPATIAL SPEECH INTELLIGIBILITY IN NOISE}

Sentence intelligibility in noise was tested with the optimized version of the Dutch Matrix test (Houben \& Dreschler, 2015)within a set-up of spatially separated speakers. The Dutch Matrix material is based on a closed speech corpus of sentences with an identical syntactical structure: "name, verb, numeral, adjective, object," for example, "Mark gives five large flowers." The accompanying Dutch Matrix noise is a stationary noise with an average power spectrum equivalent to that of the sentences. More information on the development of the Dutch Matrix material is available in Houben et al. (2014) and Houben \& Dreschler (2015). This corpus of sentences has low linguistic complexity without redundancy, making it well suited for repetitive testing (Houben et al, 2014). This speech material has been shown to be applicable for use in CI recipients (Theelen-van den Hoek et al, 2014).

The test was calibrated and administered using the Oldenburg measurement applications software package developed by HörTech gGmbH, Oldenburg, Germany (www.hoertech.de). Participants were asked to reconstruct the sentence by selecting the perceived words from 10 alternative tokens within each of the five word categories ("name, verb, numeral, adjective, object"). The participant gave their responses by using a touch screen with a visual representation of the closedset speech corpus. To force an answer, the use of an "I don't know"-button was not allowed. 
Speech was presented from a speaker at ear level at a distance of $1 \mathrm{~m}$ in the front $\left(0^{\circ}\right)$ while the stationary Dutch Matrix noise was played continuously from the same speaker (SONO), a speaker at $90^{\circ}$ on the HA-side (SONha), or a speaker at $90^{\circ}$ on the CI-side (S0Nci). This set-up is known to be able to demonstrate the benefits of binaural aiding for speech perception in noise (Avan et al, 2015).

The noise was maintained at a fixed level of $65 \mathrm{~dB}$ SPL while the first sentence started off at a level of $+5 \mathrm{~dB}$ SNR. Each sentence was scored as percentage of words correct. With each subsequent sentence, the speech level was adjusted according to an adaptive procedure that uses a logistic function (Brand \& Kollmeier, 2002) to converge at the SNR corresponding to a 50\% correct score, defined as the SRT.

Each list consisted of 20 sentences with an average test time of 5 minutes. The sequence of lists was kept constant for all participants to avoid the situation where a participant could be presented with the same list twice. To address potential learning effects (Theelen-van den Hoek et al, 2014) and familiarize participants with the task, one training list for the spatial condition S0N0 was administered prior to starting the actual test in the first listening mode (CI or CIHA). The result of this training list was excluded from the analysis.

Subsequently each of the three spatial conditions (SON0, S0Nha, S0Nci) was tested and retested for the listening condition with CI alone and with CIHA together, resulting in a total of 12 lists per participant. The listening condition (CI, CIHA) to start with was randomized between participants and completed before shifting to the other listening condition in order to avoid frequent swapping of hearing devices. The order of testing the three spatial conditions was randomized across participants but kept constant within each participant

across listening conditions and between test and retest.

In the occasional case where the adaptive procedure evoked an invalid SRT outcome, defined as a SNR result outside the range of presented levels or above $15 \mathrm{~dB}$ SNR (Kaandorp et al, 2015), the result of the affected test condition was omitted from analysis. If there were two valid outcomes, the final result per condition was calculated as the mean of test and retest.

\section{LISTENING EFFORT IN NOISE}

The set of unique sentences from the Dutch Matrix speech corpus (Houben \& Dreschler, 2015) was also used to evaluate listening effort in noise. The listening effort test is based on subjective ratings for the ease or difficulty involved in listening to speech in the presence of varying amounts of noise. This test has been 
developed within the framework of the European HearCom (Hearing in the Communication Society) project (www.hearcom.eu) to evaluate individual listening effort in a specific communication situation or with a distinct HA algorithm. The procedure has been validated as a scaling procedure at different SNRs using stationary and fluctuating background noise (Schulte et al, 2008) and has since been applied in several other studies (Brons et al, 2012; van Esch et al, 2013; Harlander et al, 2012; Luts et al, 2010). The test was administered using the Oldenburg measurement applications software package developed by HörTech gGmbH, Oldenburg, Germany (www.hoertech.de). Rating was performed using a vertical scale with 13 discrete points (seven named categories interspersed with an empty category) ranging from no effort (Score 0) to extreme effort (Score 12) (Luts et al, 2010). One randomly selected sentence from the Dutch Matrix corpus was repeated until the participant could confidently provide a self-rating of the listening effort using a vertical scale displayed on a touch screen. Listening effort was assessed for the listening condition with CI alone and with CIHA together. The listening condition to start with was randomized between participants.

The Dutch Matrix speech and stationary noise were emitted concurrently at a distance of $1 \mathrm{~m}$ in the front (SON0) from a speaker at ear level. The noise level as fixed at $65 \mathrm{~dB}$ SPL, while the speech level was set according to the participant's individual SRT resulting from the spatial speech-in-noise test in the corresponding listening condition for the spatial condition S0N0. This means that when testing at SRT level, intelligibility was by definition fixed at the 50\%-point for all participants in all modes, making it possible to evaluate listening effort on top of intelligibility. When a SRT outcome could not be determined below 15 dB SNR (Kaandorp et al, 2015), a value of $15 \mathrm{~dB}$ was assigned. Overall, six levels were set between $6 \mathrm{~dB}$ below and $9 \mathrm{~dB}$ above the participant's individual SRT in steps of $3 \mathrm{~dB}$ (SRT-6, SRT-3, SRT, SRT+3, SRT+6, SRT+9). Following one practice run in the first listening mode, each level was presented five times, resulting in a total of five sentence ratings per level. A random presentation order was applied, which differed between listening conditions and across participants. The final result per level was calculated as the mean of these five ratings.

\section{SOUND QUALITY}

The quality of bimodal speech perception was evaluated with a multidimensional questionnaire. The questionnaire was based on the inventory of quantifiable sound quality attributes as described by Boretzki (1999) within the field of hearing instrument fitting. The initial set of 21 descriptors was translated into Dutch and back-translated to the original German as well as the published English wording by two Dutch native speakers with Master degrees in German and English. Based on the Dutch translation, a pilot survey was carried out among a group of 18 bimodal 
listeners (unpublished data), who were asked to indicate those features that are most relevant to describe their hearing situation with $\mathrm{CI}, \mathrm{HA}$, and CIHA together. The 10 most frequently selected features were identified. Each of these 10 features was extracted by $35 \%$ or more of the bimodal patients and was not identified as being too difficult in terms of phrasing. The 10 most selected sound quality features (Table 2) were then used to comprise the resulting bimodal questionnaire. In his original study, Boretzki (1999)suggested a relationship between the sound quality features and the frequency region in which they are expected to be sensitive for particular acoustical modifications as a starting point for improving HA fittings. Since residual hearing in the nonimplanted ear is known to be situated mainly in the low-frequency acoustic region, one could expect the effect of bimodal hearing to appear as intensifications of the attributes full and dull while attenuating attributes that are associated with high-frequency information (Table 2).

TABLE 2. Bimodal questionnaire of sound quality features.

\begin{tabular}{|c|c|c|c|}
\hline English* & Dutch & Acoustical sensitivity* & $\begin{array}{l}\text { Test } \\
\text { order }\end{array}$ \\
\hline Voluminous or Full & Vol of Volumineus & Low frequencies & 1 \\
\hline Dull or Damped & Dof & Low frequencies & 6 \\
\hline Sharp & Scherp & High frequencies & 4 \\
\hline Bright or Harsh & Helder of Fel & High frequencies & 5 \\
\hline Tinny or Metallic & Blikkerig of Metaalachtig & High frequencies & 10 \\
\hline Shrill & Schel & $\begin{array}{l}\text { Narrow band } \\
\text { high frequency emphasis }\end{array}$ & 3 \\
\hline Hard & Hard & More likely high frequencies & 2 \\
\hline Nasal & Nasaal & More likely high frequencies & 9 \\
\hline Unclear or Blurry & Vaag of Wazig & Unspecific & 8 \\
\hline Unpleasant & Onaangenaam & Unspecific & 7 \\
\hline
\end{tabular}

* Based on the inventory of quantifiable sound quality features (Boretzki, 1999)

The questionnaire used in this study asked participants to describe "how a familiar speaker in quiet conditions sounds" to them by rating the set of 10 sound quality attributes on a linear ruler from 0 (not at all) to 10 (very) to a precision of 0.1 . Ratings were assessed for the situations with CI, with HA, and with CIHA together in this fixed order. The sound quality attributes were presented in a random order (Table 2) that was kept constant across participants. Participants were also asked to rate the overall difficulty of the questionnaire using the same ruler. 


\section{DATA ANALYSIS}

All statistical analyses were performed using IBM SPSS Statistics version 23.0.0.2. In case of missing data points, the cause was ascertained. When structural nonresponse was observed, referring to multiple untestable conditions within one participant, these cases were described and excluded from the analysis of the affected outcome measure. When data points were established to be missing at random, the mean of 100 imputations was used to

replace missing values (Graham et al, 2007). Normality was checked with the Shapiro-Wilk test and visual inspection of the outcome distributions. Overall mean pairwise differences are presented accompanied by the standard error, except where median differences were considered given the non-normal distribution of scores. Bonferroni adjustments for multiple comparisons were applied with $\mathrm{p}<.05$ indicating statistical significance.

Pairwise comparisons were performed for the dimensions of speech intelligibility in quiet and sound quality for the three listening conditions (CI-HA-CIHA). Parametric two-tailed paired-samples student t-tests were conducted for speech intelligibility in quiet. Since sound quality ratings did not appear to be normally distributed, the nonparametric two-tailed Wilcoxon signed rank test was applied to each of the 10 sound quality attributes.

When using percentage speech intelligibility scores, the room for bimodal improvement is known to be restricted if the score with $\mathrm{CI}$ alone is already high (Dorman et al, 2015). Therefore, the "normalized bimodal benefit" was also calculated for speech intelligibility in quiet by dividing the measured change by the potential change, in case of an improvement (CIHA-CI/100-CI) as well as a drop in performance (CIHA-CI/CI), and multiplying this quotient by 100 to produce an indexed score with a possible range of 100 to 100 percentage points (Zhang et al, 2013). A one-sample t-test assessed whether the normalized bimodal benefit differed significantly from zero.

The dimensions of spatial speech intelligibility in noise and listening effort were both determined using multiple measures within each test condition. To evaluate test-retest reliability, intraclass correlation coefficients (ICCs) were obtained for the two repeated measures of speech intelligibility in noise and the five repeated measures of listening effort. A one-way random ICC model (Shrout \& Fleiss, 1979) was selected since variability across participants and across repeated measurements could not be disentangled statistically. This result stemmed from the fact that different participants were assessed using different sentence lists (speech intelligibility in noise) or using different sentences (listening effort) from the Dutch Matrix speech material. 
For both the dimensions of spatial speech intelligibility in noise and listening effort, two factors were assessed. These two factors were listening condition (CICIHA) and spatial condition (SON0, SONci, SONha) for speech intelligibility in noise. For listening effort, the two conditions were listening condition (CI-CIHA) and test level (SRT-6, SRT-3, SRT, SRT+3, SRT+6, SRT+9). Therefore a two-way repeatedmeasures analysis of variance (ANOVA) was performed. Where the ANOVAs revealed statistically significant differences after applying a Greenhouse-Geisser adjustment to correct against violations of sphericity, post hoc comparisons between different levels of the factors were made with the two-tailed pairedsamples student $t$ tests.

Given the small sample size, an explorative correlation analysis using Spearman correlations without any predefined corrections was performed as a first attempt to explore the relations between the different dimensions of observed bimodal benefits. The correlation between bimodal benefits and the unaided and aided residual hearing (PTA) in the HA ear was also calculated.

\section{RESULTS}

Raw scores across the dimensions of speech in quiet, spatial speech in noise, listening effort, and sound quality are resented in two supplementary appendices. The ICC is also given for those dimensions having an outcome based on multiple measures within a test condition.

\section{SPEECH INTELLIGBBLLTY IN QUIET}

Mean CNC phoneme-scores for the CI, HA, and bimodal listening conditions are presented in Figure 2. The average score with CI was $10.3 \pm 7.1$ percentage points higher than with HA, but this difference was not statistically significant ( $\mathrm{p}=.516$ ). The difference between CI and HA was less than 10 percentage points for four participants. The CI outperformed the HA by 10 percentage points or more for eight participants. For three participants, the HA provided better monaural performance by at least 10 percentage points. The primary outcome of bimodal benefit, defined by comparing the bimodal combination CIHA to the CI alone, was found to be statistically significant with a mean improvement of $14.6 \pm 4.1$ percentage points $(\mathrm{p}=.009)$. For all the participants except two, some bimodal improvement in percentage points was observed. Listening with CIHA compared with the HA alone showed a significant improvement of $24.9 \pm 3.6$ percentage points $(\mathrm{p}<.001)$. The normalized bimodal benefit was found to be significantly different from zero by $36.2 \pm 7.0$ percentage points $(p<.001)$. 
FIGURE 2. Speech in quiet.

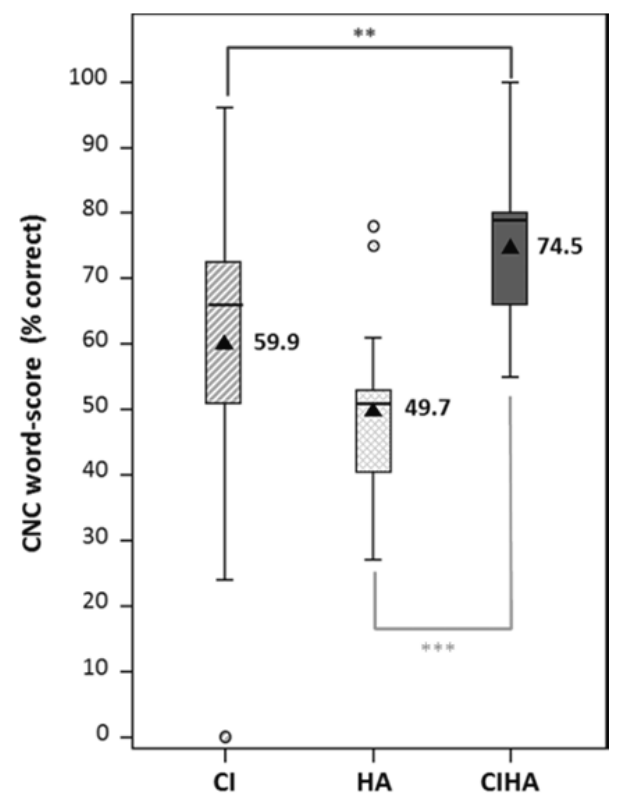

CNC phoneme-scores (\% correct) in quiet for the listening condition with $\mathrm{CI}, \mathrm{HA}$, and CIHA. Box plots represent the distribution per condition (median and interquartile range), with whiskers denoting minimum and maximum values within 1.5 times the interquartile range and circles denoting outliers. Triangles highlight the annotated mean value per condition. Asterisks denote significant differences between listening conditions $\left({ }^{* *} \mathrm{p}<.01 .{ }^{* * *} \mathrm{p}<.001\right)$. CI $=$ cochlear implant; $\mathrm{HA}=$ hearing aid; $\mathrm{CIHA}=\mathrm{CI}$ and $\mathrm{HA}$ in contralateral ears (bimodal hearing); $\mathrm{CNC}=$ consonant-nucleusconsonant.

\section{SPATIAL SPEECH INTELLIGIBILITY IN NOISE}

Where test and retest outcomes were available, the ICC for the spatial speech-innoise test was found to be 0.7 or higher over all conditions. According to the classification scale of Landis \& Koch (1977), an ICC for a single assessment greater than 0.6 is substantial and greater than 0.8 is almost perfect.

For the individual SRT outcomes in noise, one participant was unable to perform in all conditions while three participants could not perform in the $\mathrm{CI}$ alone conditions. These cases (B03, B12, B43, and B45) were therefore excluded from further analysis. Another participant (B15) featured one specific condition missing at random for which a value was imputed. Five of the remaining participants had just one outcome in one or two of the tested conditions due to the applied outcome restrictions. Given the fact that ICC values indicated a substantial reliability for the single assessment, the single assessment was taken for those specific conditions rather than the average of two assessments, as occurred in all other conditions and other participants. 
The average SRT outcomes between listening conditions (CI, CIHA) and across spatial settings (SON0, SONha, SONci) are shown in Figure 3. At an individual level, all participants demonstrated a bimodal SNR improvement in all spatial conditions, except for two participants who showed a comparable outcome in the SONha setting. The ANOVA revealed a significant main effect of the factor listening condition $\mathrm{F}(1.0,10.0)=31.7, \mathrm{p}<.001, \eta \mathrm{p}^{2}=0.76$. The main effect of spatial condition was not found to be significant, $\mathrm{F}(1.3,12.8)=2.9, \mathrm{p}=.107, \eta_{\mathrm{p}}^{2}=0.22$. However, since the interaction effect between the factors listening condition and spatial condition was statistically significant, $F(1.7,17.0)=6.5, p=.010, \eta_{p}^{2}=0.40$, the effects of these two factors were investigated separately.

FIGURE 3. Spatial speech in noise.

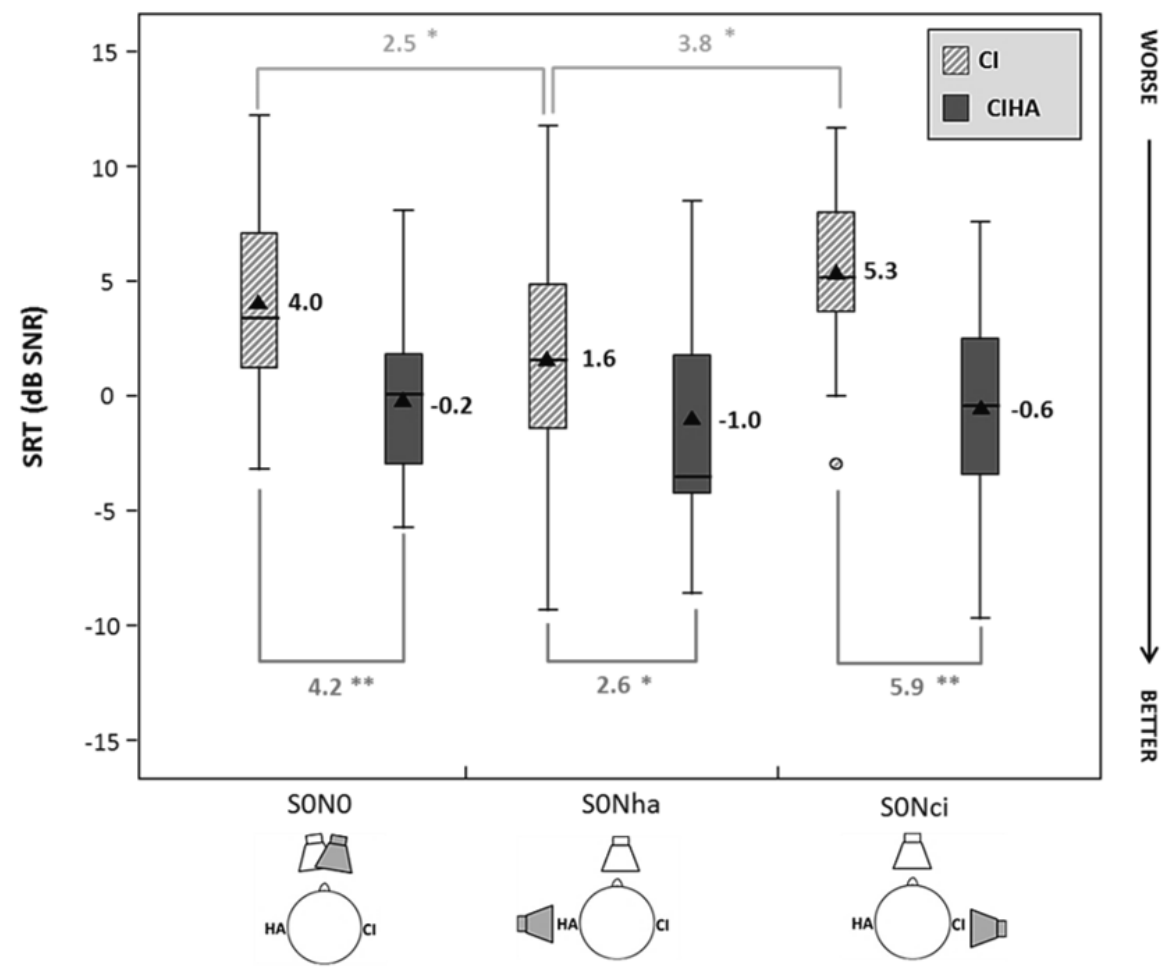

Spatial condition

Results for the spatial conditions with speech and noise from the front (SON0), speech from the front while noise from the HA side (SONha), and speech from the front while noise from the CI side (SONci). Each spatial condition was assessed for the listening condition with $\mathrm{CI}$ and CIHA. Box plots represent the distribution per condition (median and interquartile range), with whiskers denoting minimum and maximum values within 1.5 times the interquartile range and circles denoting outliers. Triangles highlight the annotated mean value per condition. Monaural effects are indicated on the top and bimodal effects in the bottom. Asterisks denote significant differences between listening conditions $\left(* \mathrm{p}<.05 .{ }^{* *} \mathrm{p}<.01\right)$. SRT $=$ speech-reception threshold; $\mathrm{CI}=$ cochlear implant; $\mathrm{CIHA}=\mathrm{CI}$ and $\mathrm{HA}$ in contralateral ears (bimodal hearing); SNR = signal-to-noise ratio. 
When comparing the SRT outcome between the bimodal (CIHA) and the unilateral (CI) listening conditions, the benefit of bimodal aiding can be determined for each spatial condition. With speech and noise coming from the front (SON0), adding the HA provided a mean significant benefit of $4.2 \pm 0.9 \mathrm{~dB}$ SNR $(\mathrm{p}=.002)$. For the setting with noise coming from the HA-side (SONha), a significant improvement of $2.6 \pm 0.7 \mathrm{~dB}$ SNR ( $p=.014$ ) was observed when the HA was added. In the condition with noise originating from the CI-side (SONci), a significant gain of $5.9 \pm 1.1 \mathrm{~dB}$ SNR ( $p=.001$ ) occurred by adding the HA. Monaural effects can be addressed by comparing the different spatial settings for the listening condition with CI alone. A significant improvement of $2.5 \pm 0.6 \mathrm{~dB}$ SNR ( $p=.011$ ) and $3.8 \pm 1.0 \mathrm{~dB}$ SNR ( $=.020)$ was seen when the noise was shifted an angle of 90 (S0N0 to S0Nha) or 180 (S0Nci to S0Nha), respectively, in the shadow of the head to the contralateral side.

When comparing spatial settings for the bimodal listening condition, one can evaluate spatial release from masking, which is defined as the benefit of spatially separating the speech and noise source. The data did not show a significant effect ( $p>.05$ ), either for the HA-side (S0N0 to S0Nha, $0.8 \pm 1.0 \mathrm{~dB}$ SNR) or the CI-side (SON0 to SONci, $0.4 \pm 1.0 \mathrm{~dB}$ SNR). Even though it is expected that the best performing ear side will vary per subject, it is not possible to evaluate spatial release from masking toward the best performing monaural side since HA-alone performance for speech recognition in noise was not assessed here.

\section{LISTENING EFFORT In NoISE}

The reliability (ICCs) of listening effort ratings for each level in each listening condition based on a single assessment ranged from fair $(>0.2)$ to substantial $(>0.6)$, whereas the average of five assessments was found to be substantial ( $>0.6$ ) to almost perfect ( $>0.8$; Landis \& Koch 1977). All participants successfully completed all rating conditions. The mean ratings across levels (SRT-6, SRT-3, SRT, SRT+3, SRT+6, SRT+9) and between listening conditions (CI, CIHA) are displayed in Figure 4. The ANOVA revealed no significant main effect of the factor listening condition $\mathrm{F}(1.0,14.0)=1.7, \mathrm{p}=.215, \eta_{\mathrm{p}}{ }^{2}=0.11$. The main effect of the factor test level was statistically significant, $F(1.3,18.2)=38.3, p<.001, \eta_{p}{ }^{2}=0.73$, as was the interaction term between the factors listening condition and test level $F(3.6,50.0)=5.3, p=.002, \eta p^{2}=0.27$. The effects of these two factors were therefore analyzed separately.

The investigation into bimodal benefit by comparing CIHA to CI revealed no significant effect over the levels SRT-6 up to SRT+3. At the level of SRT+6 , a decline of $1.0 \pm 0.4$ points lost statistical significance after Bonferroni correction $(p=.18)$. At the most favorable tested level of SRT +9 , all participants except one 
reported a reduction in listening effort of up to 4.4 points with an average reduction of $1.4 \pm 0.3$ points $(p=.007)$.

Two one-way ANOVAs demonstrated the effect of level to be highly significant $(\mathrm{p}<.001)$ whereby the rated effort decreased linearly with increasing level when listening with CI, F $(1,14)=28.3, p<.001, \eta p^{2}=0.7$, as well as bimodally, $\mathrm{F}(1,14)=58.7, \mathrm{p}<.001, \eta_{\mathrm{p}}^{2}=0.8$. For each $3 \mathrm{~dB}$ step toward a more favorable SNR level, an average of 0.8 and 1.1 points reduction in listening effort was seen for CI and CIHA, respectively.

FIGURE 4. Listening effort in noise.

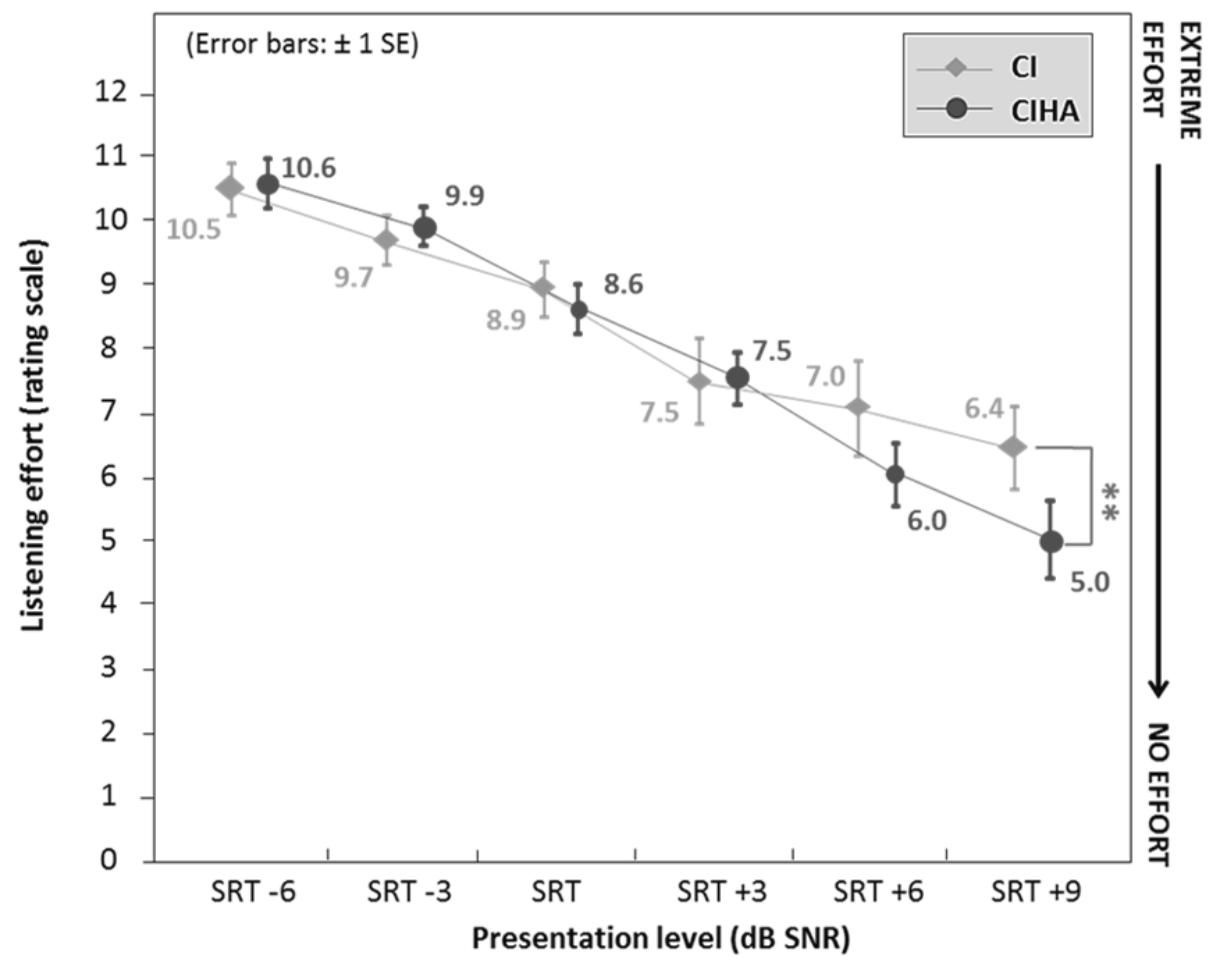

Mean listening effort ratings on a scale of 0 ( no effort )to12 ( extreme effort) for speech and noise presented from the front (SONO) when listening with CI and CIHA. Listening conditions are slightly offset on the $\mathrm{x}$ axis to improve readability. Tested levels of SRT-6, SRT-3, SRT, SRT+3, SRT+6, and SRT+9 are expressed as relative to the participant's individual SRT on the spatial speech in noise task in the corresponding condition. Asterisks denote significant differences between listening conditions $(* * \mathrm{p}<.01)$. SRT = speech-reception thresholds; $\mathrm{CI}=$ cochlear implant; $\mathrm{CIHA}=$ $\mathrm{CI}$ and HA in contralateral ears (bimodal hearing); SNR = signal-to-noise ratio. 


\section{SOUND QUALITY}

A median questionnaire score of 6.0 out of 10 was obtained for overall difficulty. No missing data occurred since all participants were able to provide a rating for all 10 sound quality attributes. Median ratings for the listening conditions (CI, HA, CIHA) across the 10 sound quality attributes are shown in Figure 5. None of the questioned attributes showed a significant difference in perceived sound quality between $\mathrm{CI}$ and HA. The bimodal condition however was rated to be significantly more voluminous (2.9 points, $\mathrm{p}=.022$ ) and brighter (2.1 points, $\mathrm{p}=.006)$ when compared with the HA condition, while being perceived more voluminous (2.3 points, $\mathrm{p}=.017$ ), less unpleasant ( 1.4 points, $\mathrm{p}=.032$ ), and less tinny (3.0 points, $\mathrm{p}=.013$ ) when compared with the $\mathrm{CI}$ alone condition.

\section{EXPLORATORY CORRELATION ANALYSIS}

The correlations between bimodal benefits on the different dimensions of speech perception are presented in Table 3. The bimodal benefit for the spatial condition SONci was found to correlate with the bimodal effect for the spatial condition SONO as well as with the normalized bimodal benefit of intelligibility in quiet. The bimodal benefit for the spatial condition S0N0 also correlated positively with the build-up of the sound quality descriptor voluminous or full. No correlations were found between the reduction of listening effort at SRT+9 and any other measure of bimodal benefit. The unaided PTA in the HA ear was found to correlate with the degree of bimodal benefit in the condition SONha and the reduction in the sound quality feature tinny or metallic. The PTA aided with HA was related to all demonstrated bimodal benefits of sound quality: an increase of voluminous or full , a decrease of unpleasant, and a reduction of tinny or metallic. These correlations indicate that lower and therefore more favorable hearing thresholds are related to more change in sound quality. 


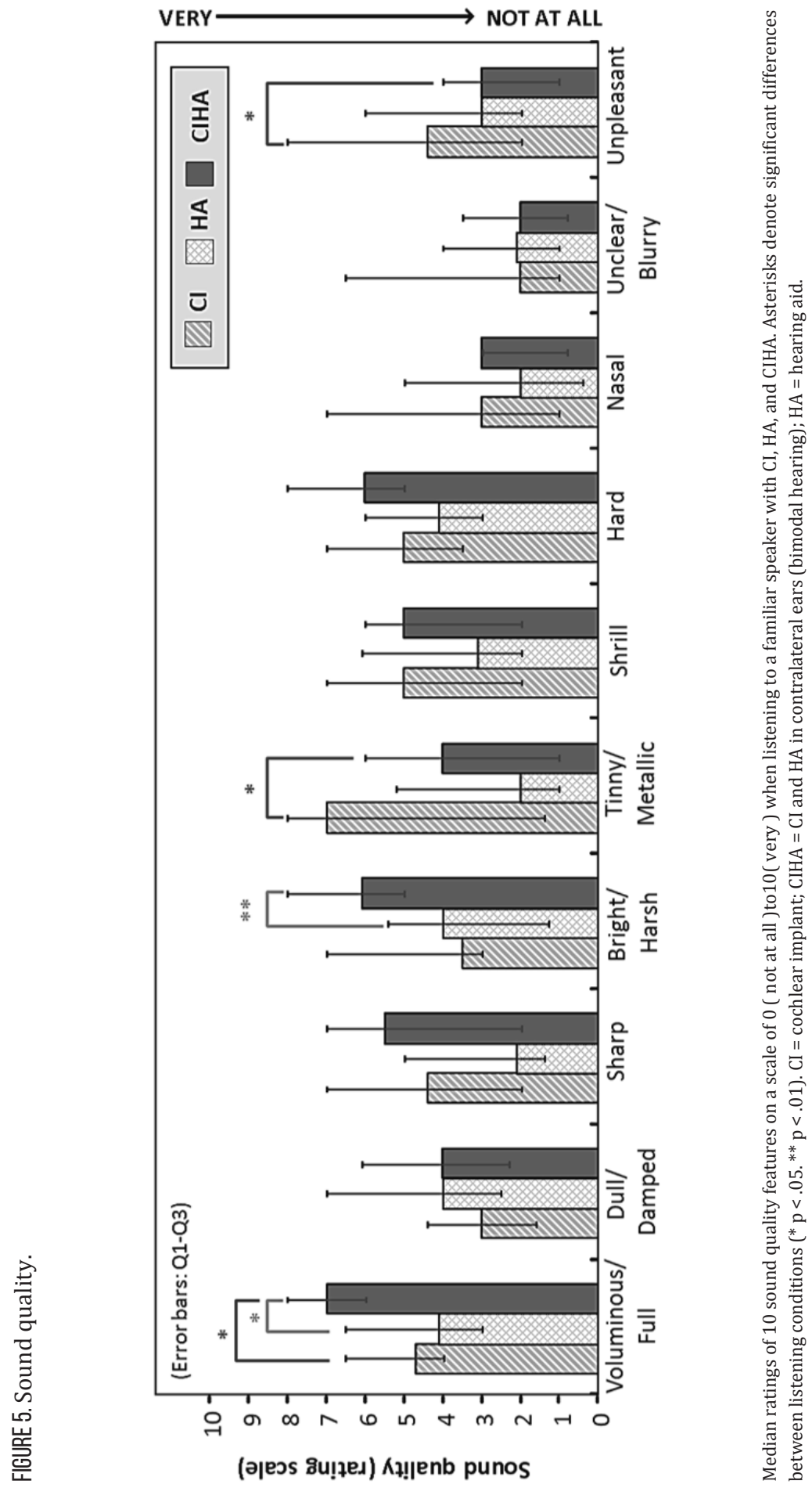


TABLE 3. Correlation Matrix of the Bimodal Benefits on Speech Perception.

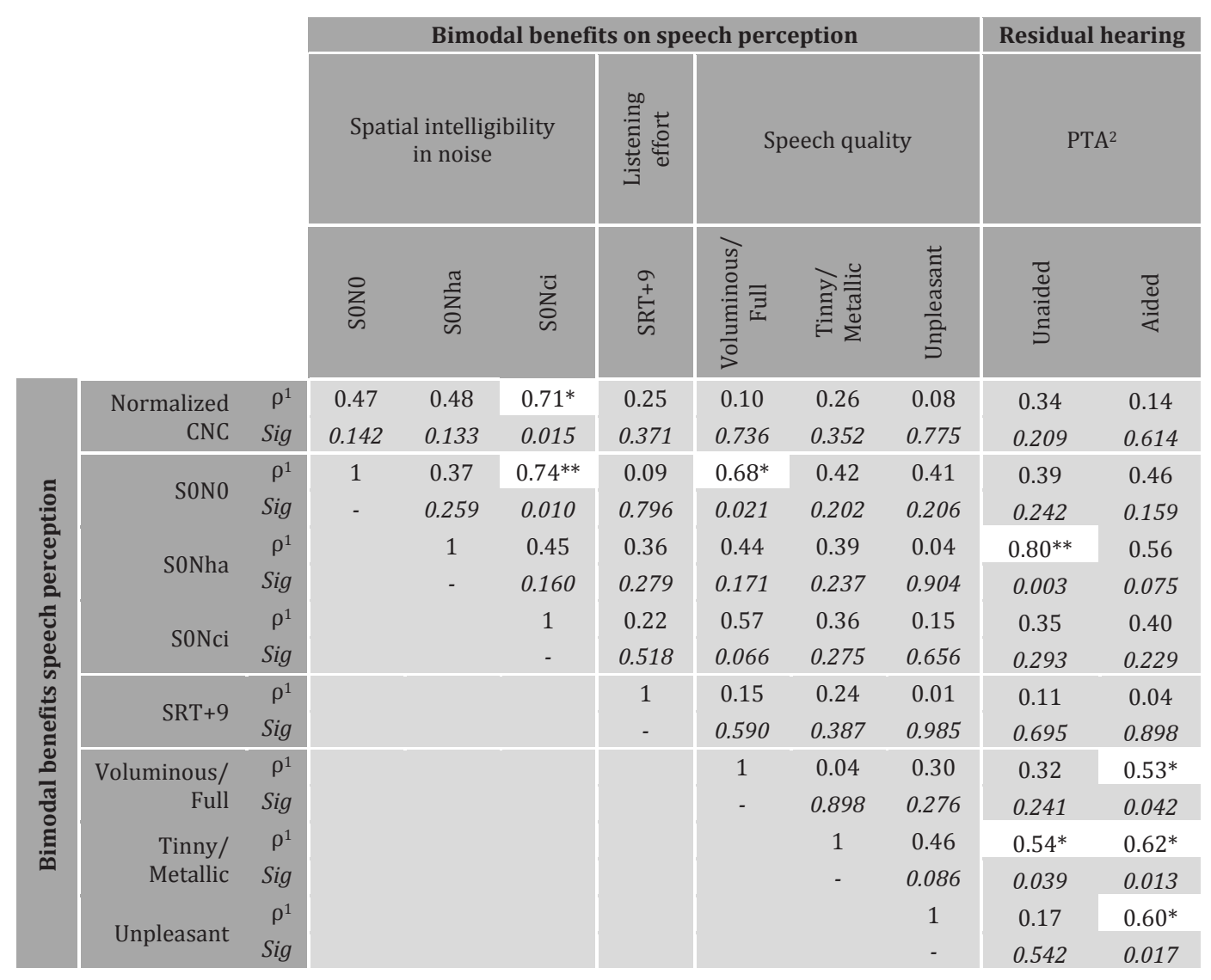

${ }_{1}^{1}$ absolute spearman rho correlation coefficients are presented, the direction of significant correlations are described in the text of the manuscript, ${ }^{2}$ pure-tone average across 250,500 and $1000 \mathrm{~Hz}$ at the hearing aid side, ${ }^{*} \mathrm{p}<0.05 ; * \mathrm{p}<0.01$ 


\section{DISCUSSION}

\section{SPEECH INTELLIGBBLITY IN QUUET}

Speech intelligibility in quiet remains the most widely used outcome measure in $\mathrm{CI}$ rehabilitation practice aside from audibility (Vaerenberg et al, 2014). In the current study, most participants (12 out of 15) scored better or about equal with CI when compared with HA alone. This finding supports the used definition of bimodal benefit as the monaural condition with $\mathrm{CI}$ alone supplemented with HA. It should however be noted that outcome differences between $\mathrm{CI}$ and HA were not found to be significant. This most likely reflects the fact that our sample included persons with a substantial degree of aidable residual hearing on the HA-side up to 1000 Hz. Compared with other studies (e.g. Gifford et al. 2014; Neuman et al. 2017), the average speech intelligibility score observed with the HA is rather high (49.5\%). The current sample is believed to be representative of the current bimodal population, as more and more patients with considerable residual hearing are being implanted. A recent bimodal population study by the same authors (Devocht et al, 2015) reported an average CNC score of $41 \%$ when aided with a HA.

Overall a significant bimodal benefit of 15 percentage points was observed, suggesting a large summation effect in quiet. Bimodal improvements on monosyllabic word scores in quiet between 10 and 20 percentage points have also been reported in other studies (Devocht et al, 2015; Dorman et al, 2008; Gifford et al, 2007; Gifford et al, 2010; Iwaki et al, 2004; Park et al, 2012; Sheffield \& Gifford, 2014; Zhang et al, 2010; Zhang et al, 2013). Dorman et al. (2008) noted that the lowest scores in the CIHA condition were near the mean score for the $\mathrm{CI}$ alone condition, as was also found here (Figure 2). Although none of the individual participants in the current population displayed reduced performance in the bimodal as compared with the CI alone listening situation, not all of them obtained a benefit. While it is considered an important basic characteristic of monaural hearing, speech perception in quiet might not be the optimal measure to assess bimodal benefit (Dorman et al, 2015; Neuman et al, 2017). Not only is speech intelligibility in quiet susceptible to ceiling effects, it might not be the most relevant measure to evaluate everyday speech perception.

\section{SPATIAL SPEECH INTELLIGIBILITY IN NOISE}

Speech intelligibility in noise is often suggested as being more relevant than testing speech intelligibility in quiet (e.g. Gifford et al. 2008). When testing in a spatial setup, bilateral and binaural benefits can be assessed (Avan et al, 2015). Commonly these benefits are attributed to the large bilateral effect of head shadow (attending 
to the input with the more favorable SNR), the smaller binaural effect of squelch (centrally combining differences across inputs) and the smallest binaural effect of summation or redundancy (combining two inputs with an identical SNR; Dillon 2012). While the exact physiological

mechanisms mediating these binaural benefits remain unclear, their presence has been demonstrated even in asymmetric hearing situations like bimodal hearing (Avan et al, 2015).

Just as in other studies in this field (Dincer D'Alessandro et al, 2015), a substantial amount of variability between bimodal participants was observed. However spatial bimodal effects were consistently found in this study, in contrast to the sometimes inconsistent and non-significant outcomes reported in earlier studies (Gifford et al, 2014; Schafer et al, 2011). In this study, all testable participants experienced some SNR improvement with bimodal aiding in multiple spatial conditions.

The poorest performance in a monaural condition was observed for speech coming from the frontal direction while noise was presented from the CI-side (SONci). This is not surprising given the fact that the CI was the primary speech input for most participants. Accordingly the largest bimodal effect of $5.9 \mathrm{~dB}$ was obtained when the HA was added in this condition. This effect reflects not only the ability to listen with both ears but also head-shadow effects on the effective speech-to-noise ratio at each ear (Dillon, 2012b). This head shadow effect has been reported to be the most robust benefit of bimodal speech intelligibility in noise (Schafer et al, 2011). Previous studies using a comparable set-up and procedure in bimodal participants noted average improvements of between 3.7 and $6.3 \mathrm{~dB}$ (Iwaki et al, 2004; Mok et al, 2006; Morera et al, 2012), which concords with the effect found in this study.

Rather than attending to one ear or the other, the bimodal benefits in the other two spatial conditions represent listeners' ability to combine information across both ears. When speech and noise both came from the front, a bimodal summation effect of $4.2 \mathrm{~dB}$ was found. When the HA input was added with an inferior SNR, a bimodal release from masking of $2.6 \mathrm{~dB}$ was found. In this spatial condition, the effect is often attributed to the binaural squelch phenomenon (Avan et al, 2015). Comparable bimodal studies refer to significant benefits of squelch and summation with an average size of 2.6 to $3.6 \mathrm{~dB}$ (Kokkinakis \& Pak, 2014; Morera et al, 2012) and 1.1 to $7.6 \mathrm{~dB}$, respectively (Ching et al, 2005; Iwaki et al, 2004; Kokkinakis \& Pak, 2014; Mok et al, 2006; Morera et al, 2012; Vroegop et al, 2017). The current study demonstrated a similar squelch effect and a rather large summation effect. It has been shown before that test procedures, material, and inclusion criteria may influence results (Dorman et al, 2015; Schafer et al, 2011). With average aided 
thresholds of $40 \mathrm{~dB}$ HL or better up to $1000 \mathrm{~Hz}$, the included participants in the current study demonstrate more potential to benefit from residual hearing capacities in the HA ear than those included in most other studies. Unlike other hearing-impaired individuals who use bilateral identical devices, bimodal listeners are predefined to have asymmetric hearing. This can lead to significant benefits when the low-frequency acoustic ingredients of the HA ear are complementary to the characteristics of electric hearing with a CI. This complementary component of redundancy, probably fulfilled by the monaural extraction of fundamental pitch information (Avan et al, 2015; Brown \& Bacon, 2010; Qin \& Oxenham, 2006), seems to play a major role in the benefits of bimodal aiding (Schafer et al, 2011). Complementarity could therefore be responsible for the large bimodal summation effect. On the other hand, it is possible that the comparatively small bimodal squelch effect simply reflects the associated degree of summation instead of reflecting the true binaural integration of interaural level and time differences. It is namely known that due to timing and loudness inconsistencies across CI and HA devices and the limited frequency overlay between listening modes, the bimodal use of interaural differences in itself is limited (Francart \& McDermott, 2013).

Although spatial speech-in-noise testing with an adaptive SRT-procedure is a wellestablished method to demonstrate bimodal benefits, it demands a specific set-up and extensive test time. Furthermore some CI recipients are unable to perform when testing speech-in-noise (Kaandorp et al, 2015; Schafer et al, 2011). In the current study, 4 out of 15 participants with incomplete results in noise could not be included in the corresponding analysis. Not surprisingly, it was seen that those participants were the poorest performers on CI speech intelligibility in quiet, with word scores of less than 50\% (Appendix 1). Although the closed-set sentence material used in this study has proven its applicability in CI recipients (Theelenvan den Hoek et al, 2014), it is not surprising that an SRT-outcome in noise cannot be determined for CI recipients with speech recognition performance below $50 \%$ correct in quiet. In three of the excluded participants meningitis was assessed to be the cause of hearing loss (Table 1). It has been shown that meningitis-associated cochlear ossification can result in less favorable CI outcomes when implanted at a later age (Blamey et al, 2012; Durisin et al, 2010; Kraaijenga et al, 2015; Waltzman et al, 1995). Three out of four participants who were excluded from the final spatial speech in noise data analysis were able to perform in the bimodal condition but failed to reach an outcome below $15 \mathrm{~dB}$ SNR when listening with their CI alone (Appendix 1). Therefore the presence of bimodal benefit could be hypothesized even though the actual degree could not be determined. Excluding these cases, if anything, may have resulted in an underestimation of the bimodal benefit in noise. Other studies often deal with the issue of not testable participants in noise by not including them in the first place (e.g. Buechner et al. 2014; Cullington \& Zeng 2011; 
Nelson \& Jin 2004). This, however, introduces a selection bias with the population of bimodal recipients not being fully represented. The current study aimed to gain more insight into the applicability of this outcome measure in a relevant bimodal sample and the characteristics of non-testable participants. Other measures of speech perception were also introduced here to target other dimensions of bimodal benefit applicable to the total range of bimodal recipients.

\section{LISTENINGEFFORT}

The proposed extended measures of bimodal speech perception could be obtained for all included participants. Provided that the relative SNR-levels were fixed to a maximum for those who were unable to perform speech intelligibility testing in noise, an overall significant release of listening effort up to 1.4 points could be demonstrated at higher individualized SNRs.

The intention of setting levels relative to the individual SRT was to enable the perceptual extension of listening effort per se, instead of an iteration of measuring subjective intelligibility (Preminger \& Van Tasell, 1995a; Preminger \& Van Tasell, 1995b). It has been reported, that reduction in perceived listening ease can be influenced by the listener's awareness of decreasing in speech understanding (Feuerstein, 1992). In the current study, which assessed effort in addition to intelligibility, exploratory correlation analysis suggested the benefit of listening effort to be a dimension in itself, since no clear relations with any of the other bimodal benefits were found (Table 3). It should however be noted that even though a correction was applied for the individual level of intelligibility at SRT for the corresponding listening condition, both listening conditions (CIHA and CI) do not necessarily have to follow the same course of intelligibility across levels below and above SRT. Further research is then warranted to shed more light on the differences in psychometric functions between bimodal listening conditions.

The results demonstrated a reduced rated effort at more favorable SNRs. At higher individualized SNRs, reasonably good levels of speech intelligibility of $50 \%$ up to $100 \%$ are expected, while noise still challenges the listening situation (Rönnberg et al, 2014). It has been reported that listening effort can be reduced even if speech intelligibility is at its maximum level (Klink et al, 2012a), since limited abilities like degraded spectral resolution can result in increased effort even when intelligibility is at $100 \%$ (Winn \& Edwards, 2013). Listening effort therefore has been demonstrated to be a sensitive measure to assess the transmission of speech, especially at high levels of performance in noise (Morimoto et al, 2004). It has, for example, been suggested that the effect of noise modulations may only be apparent at better SNRs (Rönnberg et al, 2010). Research into the cognitive capacities related to speech perception indicates that at poor SNRs, working memory 
capacity plays a major role, while at more favorable SNRs, the importance of the executive function of updating gains importance (Ellis \& Ronnberg, 2014; Rönnberg et al, 2014; Rudner et al, 2011). Given the within-subjects design of the current study, basic cognitive capacities of tested participants did not differ between listening conditions. However it can be imagined that when more complementary information becomes available by adding the acoustic to the electric input, the executive functions of processing and updating this information, in particular, experience a lower workload while the amount of extracted information to be stored in memory stays the same given the same stabilized level of intelligibility. Just as in other studies (Rudner et al, 2012; Schulte et al, 2008), the amount of effort decreased at higher SNRs. But while effort stagnated at higher levels when listening with a CI alone, a further decline was observed for the bimodal listening situation. It should however be noted that even with bimodal aiding, the rated effort was far from zero since it dropped only to 5 on a 12-point scale.

A simple scaling method at individualized SRT levels demonstrated here to be a simple and fast way to address bimodal listening ease. Testing all levels with the used scaling procedure required about 15 minutes time, leaving a single level testable within only a few minutes.

\section{SOUND QUALITY}

Even though the feedback provided by bimodal users indicates an improvement in sound quality experience, this study was the first to specifically quantify bimodal sound quality for multiple attributes. With this intention, a questionnaire based on known sound quality descriptors (Boretzki, 1999) was proposed and well received by bimodal users. Adding a contralateral HA in addition to a CI resulted not only in a less tinny sound (3.0/10), an effect found earlier (Christal, 2012), but also a more voluminous (2.3/10) and less unpleasant (1.4/10) sound quality experience. These outcomes closely reflect the experiences heard from clinical practice within the field of bimodal hearing (e.g. Potts et al. 2009). They are also in line with a study, demonstrating that in normal-hearing listeners, binaural input delivers primarily more fullness and a better overall impression when compared with monaural hearing (Balfour \& Hawkins, 1992). As shown in Table 2, and is known from the amount of residual hearing in the HA-ear (Figure 1), these descriptors relate to a reduced dominance of components in high-frequency regions and added components in low-frequency regions. Furthermore, the exploratory correlation analysis suggests that voluminous benefit is associated with benefit in the condition SONO (Table 3), which is not surprising in the light of the known phenomenon of loudness summation (Moore \& Glasberg, 2007). 


\section{Residual HeARING}

With inclusion criteria broadening over the years, more candidates with relatively more residual hearing have become CI recipients (Gifford et al, 2010; Hughes et al, 2014). It has been shown that low-frequency residual hearing plays an important role in bimodal hearing (Büchner et al, 2009; Illg et al, 2014; Kong et al, 2005; Mok et al, 2010). Many previous studies, however, did not find a significant correlation between measures of bimodal benefit and residual hearing thresholds in the HAear (Beijen et al, 2008; Ching et al, 2006; Dincer D’Alessandro et al, 2015; Litovsky et al, 2006; Mok et al, 2006; Veugen et al, 2015; Yoon et al, 2012b). Others on the other hand have suggested that more residual hearing leads to more bimodal benefit (Dorman et al, 2015; Firszt, 2008; Zhang et al, 2013).

Although this group of participants has fairly favorable residual hearing, a clear correlation could not be discerned between hearing thresholds and the largest effects of speech intelligibility or listening effort in noise. It should be kept in mind, however, that due to small sample size, we are confronted with limited statistical power. At the same time, other studies have not been able to fully explain the specific origins of the large intersubject variations in bimodal benefits. Explanations have been proposed such as spectral resolution of the HA ear (Zhang et al, 2013) or differences in the characteristics of the two ears (Yoon et al, 2015).

\section{LIMITATIONS AND RELEVANCE}

Prior studies have evaluated bimodal speech intelligibility (Gifford et al, 2014; Gifford \& Dorman, 2012; Kokkinakis \& Pak, 2014; Morera et al, 2012; Schafer et al, 2011). The novel contribution of the current study lies in the fact that it aimed at pointing toward the content and applicability limitations of intelligibility outcome measures. This was demonstrated by evaluating a bimodal test battery within a recent clinical sample introducing other outcome measures such as listening effort and sound quality. Indeed the results could illustrate that listening effort and sound quality were outcome measures of bimodal benefit on top of intelligibility, which are clinically testable across the whole range of bimodal participants, in contrast to the more complex research originated measure of spatial intelligibility in noise. Like other CI studies into the benefits of bimodal aiding (Schafer et al, 2011), the present study had a limited sample size. The relationship between the tested dimensions of bimodal benefit and residual hearing (Table 3) could therefore only be examined with simple correlation analysis, given the number of assessed variables and limited power. This precludes making strong conclusions and generalizing the obtained results to the total population of bimodal recipients. The current sample can be considered representative of bimodal participants 
being currently fitted, having CI speech intelligibility scores in quiet ranging from 0\% up to 96\% (Dorman et al, 2008; Dorman et al, 2015; Zhang et al, 2013)and aidable hearing in the HA ear up to $1000 \mathrm{~Hz}$ on average (Hughes et al, 2014; Yoon et al, 2012b). So even though any conclusions drawn must be considered with caution, the results make a relevant contribution to the current field of bimodal aiding, while providing suggestions for future research. Furthermore, the directions resulting from the presented set of outcome measures may also extend toward related research areas and patient populations such as combining input across equally aided ears (bilateral HAs or bilateral CIs) or combining acoustic and electric input within the same ear (electro-acoustic stimulation). Nevertheless the test battery should be further evaluated in a larger set of patients and settings to gain more insight into the relationship between benefits, the mechanisms underlying these benefits, and ways to optimize them.

\section{CONCLUSION}

This study was conducted to extend the common measures of speech perception to include other dimensions relevant to the current population of bimodally aided CI users. Measures of subjective listening effort and sound quality were applied in clinical practice, in addition to the more complex testing of spatial speech in noise. Significant benefits of combining the CI with a conventional HA in opposite ears were observed across tested speech perceptual dimensions. As in other studies, all tested bimodal improvements of speech intelligibility were obtained in different spatial settings of speech and noise. Moreover a reduction of listening effort was present at the highest SNRs tested. Furthermore it was established that bimodal hearing reflected a more voluminous, less tinny, and more pleasant sound experience. Listening effort and sound quality suggested complementary outcomes. Therefore it is advisable to take various dimensions of speech perception into account when assessing bimodal benefit in current clinical CI populations. 
APPENDIX 1.

Raw scores on speech in quiet. spatial speech in noise and listening effort in noise.

Available online: http://journals.sagepub.com/doi/suppl/10.1177/2331216517727900

APPENDIX 2.

Raw scores on sound quality.

Available online: http://journals.sagepub.com/doi/suppl/10.1177/2331216517727900

\section{ACKNOWLEDGMENTS}

The authors are grateful to all participants for their time and effort during testing. The authors thank M. Boretzki for his permission and suggestions on adopting the sound quality attributes as published by Boretzki (1999) into the sound quality questionnaire used in this study. The authors acknowledge the assistance of M. Truyens and G. van Hees in the translation process of the sound quality questionnaire, the contribution of F. Scherf (employed by Advanced Bionics) during the protocol development of this study, and the English editing services provided by M. Chenault in finalizing the manuscript. Preliminary portions of these data were presented at the $8^{\text {th }}$ international Symposium on Objective Measures in Auditory Implants (October 2014, Toronto, Canada) and the $18^{\text {th }}$ Jarestagung of the Deutsche Gesellschaft für Audiologie (March 2015, Bochum, Germany).

\section{DISCLOSURE OF INTEREST}

This work is supported by a research grant from Advanced Bionics Inc. to MUMC+ in this investigator-initiated study. Statistical support was made possible by a grant from the Dutch Heinsius-Houbolt Foundation. J. C. holds a scientific post in the Advanced Bionics European Research Center. The study was designed in cooperation between MUMC+ and Advanced Bionics. Data collection, analysis, and the decision to publish were all solely accounted for by MUMC+. The work presented in this manuscript is the intellectual property of MUMC+. 



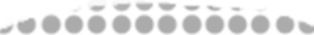

-

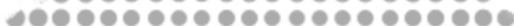

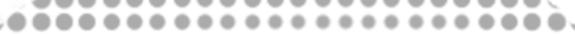

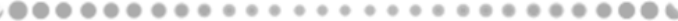

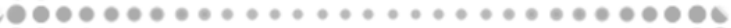

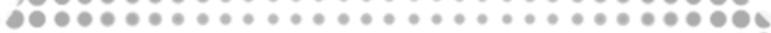



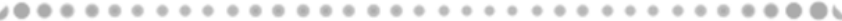

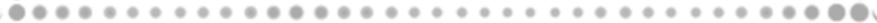

เ

분

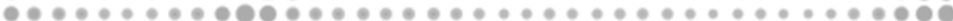

,

,

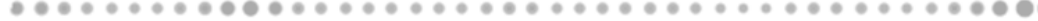

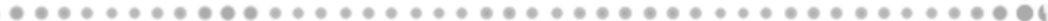

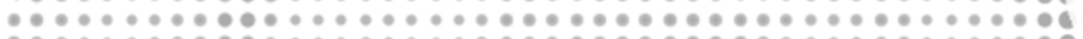

$\because 2$ MONAURAL BEAMFORMING IN a $\therefore$ EFFECT OF CAJSYMMETRICDIRECTIVITY AND NOISE TYPE

논

,

,

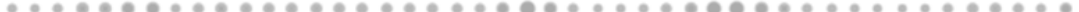

.

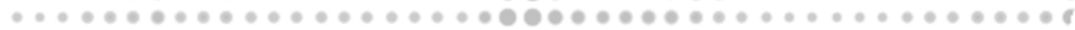

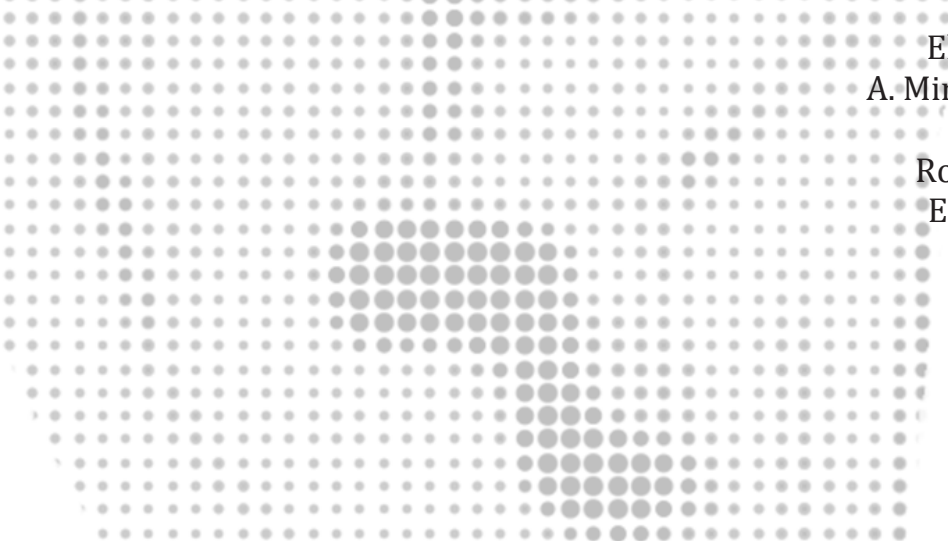

Elke M.J. Devocht

A. Miranda L. Janssen Josef Chalupper Robert J. Stokroos Erwin L.J. George

PLOS ONE, 2016, 11(8): e0160829

DOI:10.1371/journal.pone.0160829 
The objective of this study was to evaluate monaural beamforming in bimodally aided cochlear implant (CI) users. The study enrolled twelve adult bimodal listeners who were uniformly fitted with the same CI speech processor and HA, giving access to an identical monaural beamformer in both ears. A within-subject repeated measures design evaluated three directional configurations [omnidirectional, asymmetric directivity (in CI alone) and symmetric directivity (in both CI and HA)] in two noise types [stationary and fluctuating]. Bimodal speech reception thresholds (SRT) as well as listening effort ratings were assessed in a diffuse noise field. Results show that monaural beamforming provided substantial benefit for speech intelligibility in noise for bimodal listeners. The greatest benefit occurred when monaural beamforming was activated symmetrically in both CI and HA. Directional benefits were similarly observed in stationary and fluctuating noise. Directivity did not contribute to less listening effort in addition to improvement in speech intelligibility. Monaural beamforming does not bridge the gap between bimodal and normal hearing performance, especially in fluctuating noise. Results advocate further bimodal co-operation. 


\section{INTRODUCTION}

Although most cochlear implant (CI) recipients can achieve high levels of speech intelligibility in quiet (Krueger et al, 2008), understanding speech in the presence of noise or competing talkers remains a major challenge (Fetterman \& Domico, 2002; Noble et al, 2008a). Useful input to both ears is a way to improve speech perception in noise. Both bilateral CIs and bimodal hearing, referring to the combination of a $\mathrm{CI}$ and a conventional hearing aid (HA) in opposite ears, are known to improve intelligibility in noise by offering access to bilateral and binaural cues (Blamey et al, 2015; Ching et al, 2007; Schafer et al, 2011). CI candidacy criteria are expanding (Gifford et al, 2010) and now include patients with aidable residual hearing. Bimodal fitting therefore has become wellestablished clinical practice (Scherf \& Arnold, 2014) and higher bimodal hearing aid retention rates have been reported (Devocht et al, 2015). It has been shown that by combining modalities, the limits of electrical hearing can be complemented by low-frequency information retrieved from the acoustic ear (Büchner et al, 2009; Illg et al, 2014; Sheffield \& Gifford, 2014).

An alternative approach to enhance speech intelligibility in noise is to improve the signal-to-noise ratio (SNR) before sound is offered to the ear. Given that interfering sources are often spatially separated, a directional microphone system may be applied to focus on the target speech while attenuating noise from other directions (Ricketts \& Mueller, 1999). Creating microphone directionality is often referred to as beamforming (Peterson \& Zurek, 1987). Modern beamformers make use of multi-microphone arrays (Soede, 1993) located at the same (monaural) or across ear sides (binaural) (Klasen \& Moonen, 2005) and can function in a fixed or adaptive manner (Kompis \& Dillier, 2001). Directional microphone systems, available in HAs since the 1970s (Sung et al, 1975), substantially improve speech intelligibility in noise (Amlani, 2001; Bentler, 2005; McCreery et al, 2012). Since 2005 also CI recipients have been able to benefit from monaural adaptive beamforming (Brockmeyer, 2011; Buechner et al, 2014; Chung et al, 2006; Geissler \& Arweiler, 2014; Gifford \& Revit, 2010; Hersbach et al, 2012; Spriet et al, 2007; Wouters \& Vanden Berghe, 2001).

Given that directional microphone systems are now available for both HA and CI, and that benefits provided by bimodal hearing are expected, it can be hypothesized that the two approaches are complementary in improving speech intelligibility in noise. An unaddressed question related to the bimodal application of directivity is whether to activate beamforming in both CI and HA or only in the primary speech input, which is often the CI. When a directional microphone is activated in one ear while an omnidirectional microphone is used in the other ear, it is referred to as an 
asymmetric directional fitting (Mackenzie \& Lutman, 2005) (Fig 1). Given that an asymmetric hearing situation is often predefined when combining CI and HA in opposite ears, the evaluation of directional symmetry is of particular interest in bimodal hearing.

FIGURE 1. (A)symmetric directivity.

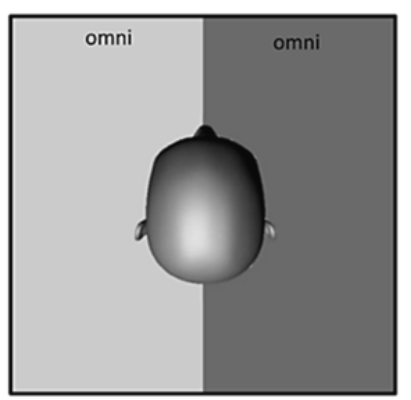

A. Bilateral Omnidirectional

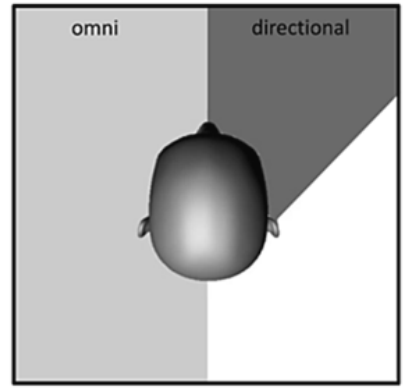

B. Asymmetric Directional

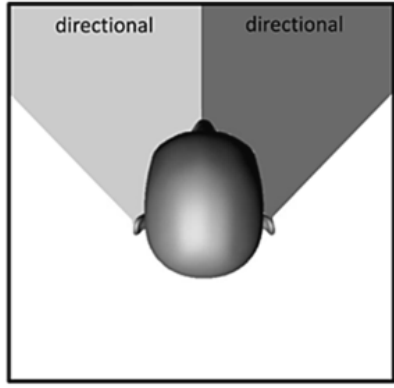

C. Symmetric Directional

Schematic illustration of bilateral omnidirectional (A), asymmetric directional (B) and symmetric directional (C) configurations. Online available at: doi:10.1371/journal.pone.0160829.g001

The masking efficiency of noise is known to depend on the availability of temporal gaps and fine structure, as well as the degree of perceptual similarity between target and interferer (Francart et al, 2011). Speech-in-noise testing is traditionally performed in stationary noise in the absence of the abovementioned characteristics. Non-stationary and modulated maskers are however considered as being more representative of everyday listening situations (George et al, 2007; Kramer et al, 1996). Bimodal users still have some limited access to low frequency hearing through the use of a contralateral HA. It could therefore be argued that acoustic HA use, to some extent, facilitates listening in the temporal gaps and using temporal fine structure which cannot occur via electric CI stimulation (Francart \& McDermott, 2013). Moreover, directional microphones enhance the SNR available to the ear theoretically giving more access to temporal information of speech within background noise. Directional microphones could then, in particular, be expected to improve performance in fluctuating noise (Spriet et al, 2007).

Measurements of speech perception in noise seldom extend beyond intelligibility. It is however known that in challenging auditory environments it can be 'easier' or 'harder' to listen to speech even for identical levels of intelligibility (Klink et al, 2012b; Klink et al, 2012a). Background noise namely can make speech communication tiring and cognitively taxing, especially for individuals with hearing impairment (Rudner \& Lunner, 2014). Sometimes listening difficulty ratings can evaluate speech transmission more accurately and sensitively than intelligibility scores, especially at high performance levels (Morimoto et al, 2004). 
Even though improvement in intelligibility has not been seen, noise reduction algorithms have proven to reduce listening effort (Sarampalis et al, 2009) and free up cognitive resources for other tasks ( $\mathrm{Ng}$ et al, 2013). Also directional microphone systems have previously been described as easing listening effort in some HA studies (Cord \& Walden, 2007; Wu et al, 2014).

\section{CURRENT STUDY}

The current study was designed to evaluate the performance of a monaural beamformer in bimodal listeners. It was hypothesized that, for speech perception in noise, symmetric directional fitting (monaural beamforming in both $\mathrm{CI}$ and $\mathrm{HA}$ ) could provide more benefit than asymmetric fitting (monaural beamforming in CI only). Hence both directional configurations were compared to the omnidirectional standard for the primary outcome of speech intelligibility and the secondary outcome of listening effort in the presence of a stationary as well as a fluctuating masker.

\section{Materials AND MethodS}

\section{ETHICS}

The study was approved by the local Medical Ethical Committee (Maastricht University Medical Center, NL51559.068.14), registered in the Dutch National Trial Register (NTR4901) and conducted in accordance with the Declaration of Helsinki. All bimodal participants provided written informed consent prior to participation and received compensation for participation and travelling expenses.

\section{Participants}

The bimodal study group consisted of twelve adult bimodal listeners ( 8 male/4 female; mean age 64.6 years, SD 14.2 years, range 23-77 years). All participants were Dutch speaking and had at least 6 months of regular experience with a CI speech processor of the brand Advanced Bionics (AB) (Valencia, USA). In the contralateral ear all participants had to use a conventional HA for at least $50 \%$ of the daytime. The aided phoneme score in quiet for the bimodal situation had to be at least $50 \%$ to ensure ability to participate in speech-in-noise testing (Theelen-van den Hoek et al, 2014) and be included as a participant in the current study. Details on the individual hearing situation of the bimodal participants are presented (Table 1). To estimate the effect of noise type in the speech-in-noise test, a normal hearing group was considered $(n=7$, age 27.3 years, SD 4.5 years, audiometric thresholds_20 dB HL) as reference. 


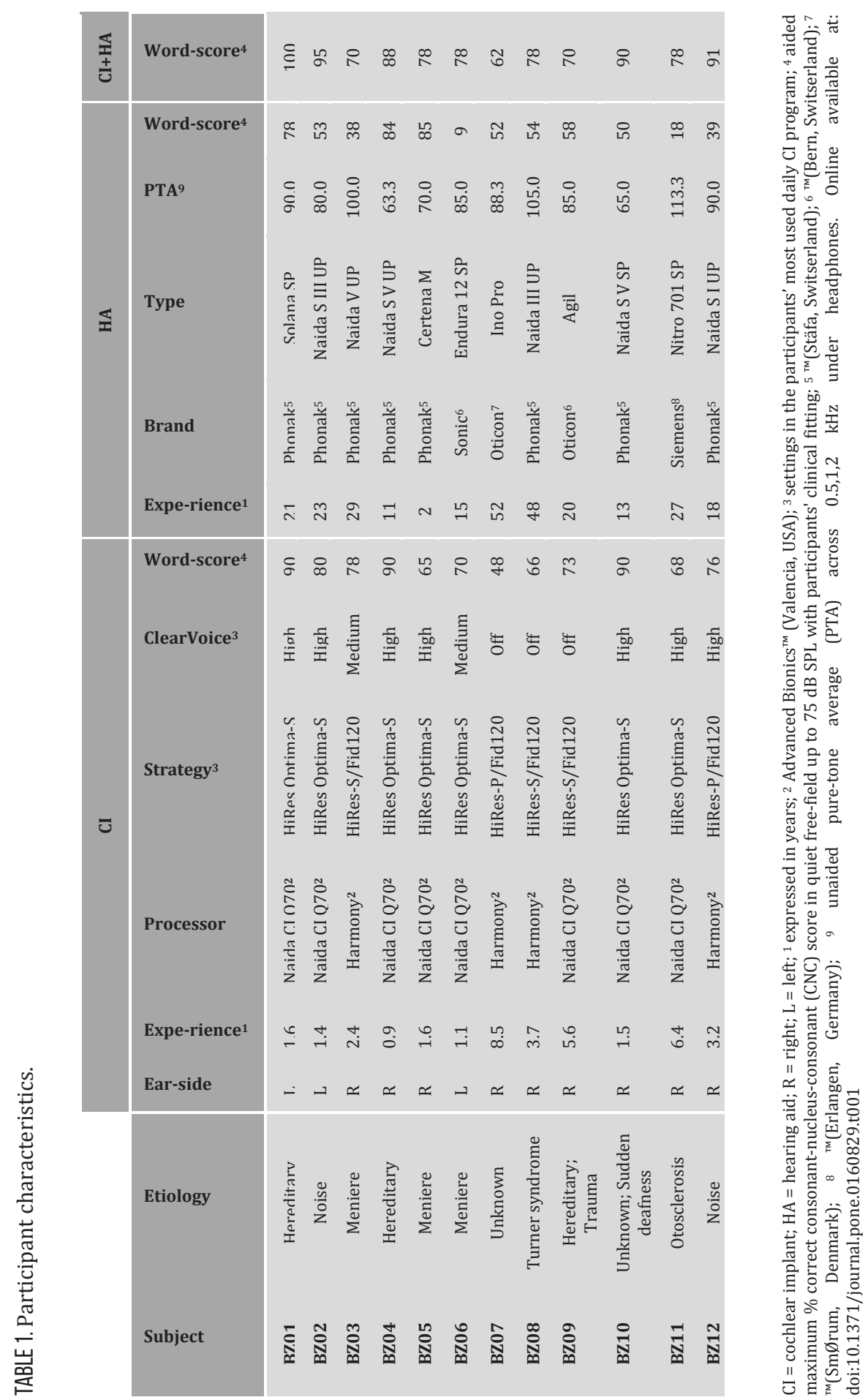




\section{MonaURAL BEAMFORMING}

The UltraZoom ${ }^{\mathrm{TM}}$ system was used since this directional system is identically available in CI processors by AB (Buechner et al, 2014; Geissler \& Arweiler, 2014) as well as in HA's by Phonak due to the collaboration between these two manufacturers within the Sonova group (Stäfa, Switserland). This directional system is a monaural beamformer based on an array of two omnidirectional microphones. Sound attenuation for the back hemisphere is adaptively steered in a frequency specific manner depending on the noise source orientation (Elko \& Pong, 1995).

\section{DEVICE FITTING}

All participants were fitted in the context of the study with the exact same CI speech processor and HA in order to avoid variability of devices and microphone effectiveness (Keidser \& Seymour, 1999). The participant's daily CI program (based on a HiRes Fidelity $120^{\text {тм }}$ or Optima ${ }^{\mathrm{TM}}$ processing strategy, Table 1), was transferred without changing basic map parameters from their everyday processor into the selfsame study speech processor (Naida CI $\mathrm{Q}^{\mathrm{T}}{ }^{\mathrm{TM}}, \mathrm{AB}$ ). The optionally activated noise reduction algorithm (ClearVoice ${ }^{\mathrm{TM}}$ (Buechner et al, 2010; Kam et al, 2012), Table 1) was converted accordingly. No interaction exists between this algorithm and the investigated beamformer (Buechner et al, 2014). In the opposite ear, all participants were uniformly fitted with the same hearing aid (Naida Q90 $U^{\mathrm{TM}}$, Phonak) to their own closed earmold. The HA's proprietary formula (Adaptive Phonak Digital) was used to calculate the prescribed acoustic gain based on the participant's residual hearing thresholds. The optimized frequency response and aligned compression of the Bimodal Formula (Chalupper et al, 2013) was applied to enhance fitting for the bimodal situation. All other HA processing features, aside from feedback cancellation, were deactivated to avoid interactions. Following a short acclimatization period, gain settings were individually fine-tuned based on the participant's feedback of interaural balance or comparability with their daily fitting.

\section{STUDY DESIGN}

A within-subject repeated measures design was applied (Fig 2). Speech intelligibility performance (I) was first assessed followed by listening effort (II). Each outcome measure was tested for the bimodal situation in three directional configurations $(1,2,3)$ and two masking noises (A and $B$ ), resulting in six test conditions per outcome. To control for sequencing, test conditions were randomised across subjects for each outcome measure separately using a balanced Latin Square design. 


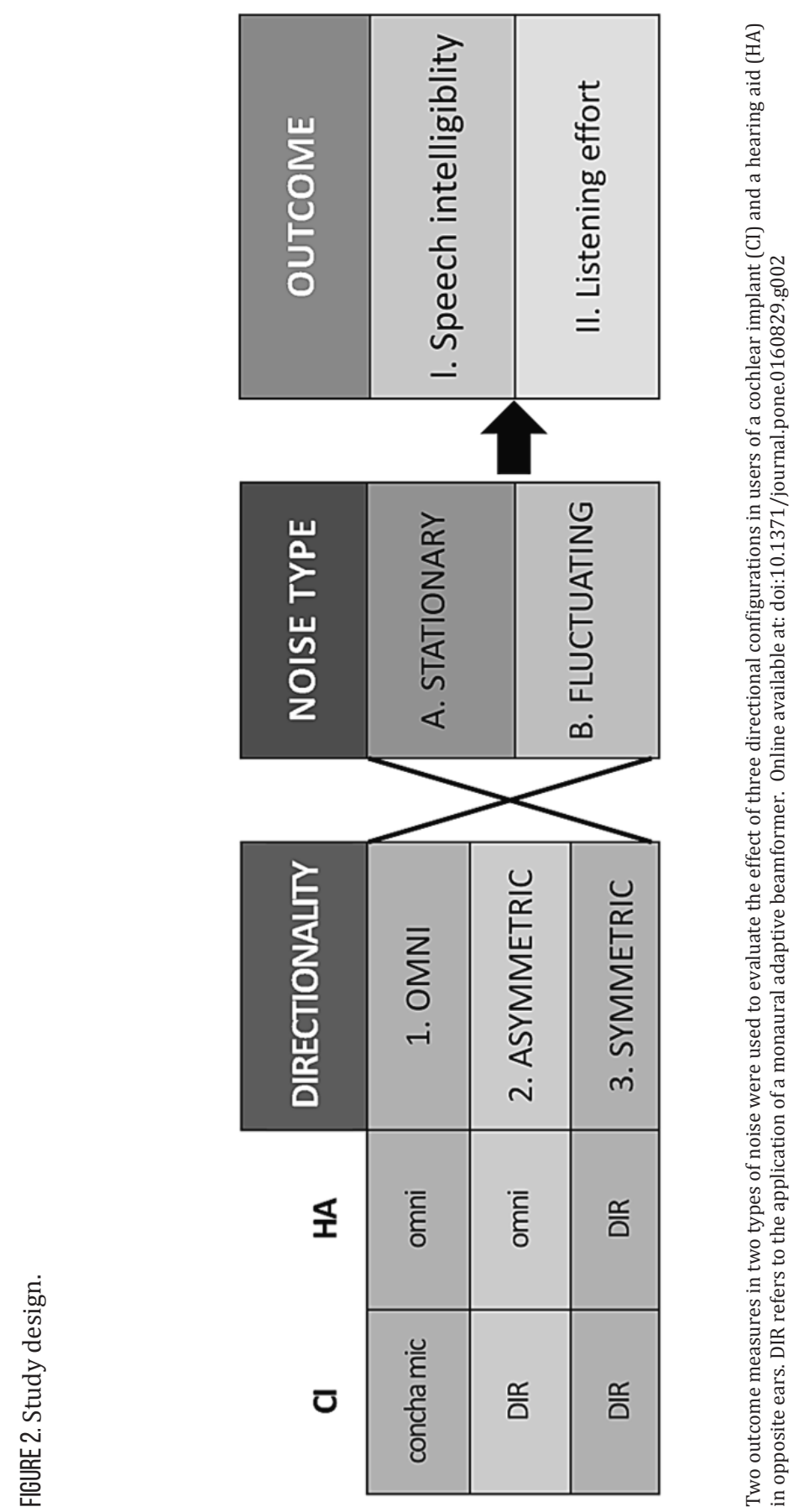




\section{DIRECTIONAL CONFIGURATION}

Three directional configurations were single-blindly evaluated: omnidirectional (1), asymmetric (2) and symmetric directivity (3). To switch and verify the directional configurations according to the condition to be tested, the researcher used a remote control on the $\mathrm{CI}$ as well as the HA. Participants were not informed about the directional settings of their devices during testing.

The reference condition was the omnidirectional setting with the standard microphones in CI and HA. For the HA the basic standard is an omnidirectional behind-the-ear microphone. For the CI processor, the default microphone is the T$\mathrm{Mic}^{\mathrm{TM}}$ : an omnidirectional microphone positioned in the pinna (Frohne-Büchner et al, 2004). In the symmetric configuration, directivity (DIR) was switched on in the $\mathrm{CI}$ as well as the HA, resulting in the same monaural beamformer applied bimodally.

In order to keep total test time within participants' concentration span, only the single most relevant asymmetric directional setting was included. For CI-recipients the CI-side in general is assumed to serve as the primary input for speech intelligibility. Furthermore it has been observed that severe hearing impaired listeners are less likely to use a directional microphone program on their HA (Keidser et al, 2008) since they have a significantly lower potential in directional benefit when compared to listeners with moderate hearing loss (Ricketts et al, 2005). Therefore the most clinically relevant asymmetric configuration of primary interest in this study consisted of activating the monaural beamformer (DIR) at the CI-side while keeping the HA at the omnidirectional setting.

\section{SPEECH INTELLIGIBILITY}

To assess speech intelligibility in noise (I), the optimized version of the Dutch Matrix test (Houben \& Dreschler, 2015) was used. This speech-in-noise test is based on a closed speech corpus of sentences with the same fixed syntactical structure founded on five word categories 'name, verb, numeral, adjective, object'. For example ' Mark gives five large flowers. ' More details on the Dutch Matrix test can be found in publications by Houben et al (Houben et al, 2014; Houben \& Dreschler, 2015). The corpus is by design well suited for repetitive testing and has proven to be applicable for use in cochlear implant recipients (Theelen-van den Hoek et al, 2014). The test was administered as a closed-set. The participants provided responses on a digital touch screen displaying a matrix containing the ten alternative tokes within each of the five word categories. Since the use of an 'I don't know'-button was not allowed, the participant was forced to make a choice within each of the five categories to reconstruct the perceived sentence. 
The noise was kept at a fixed overall level of $65 \mathrm{~dB}$ SPL, while the speech level started off at $+5 \mathrm{~dB}$ SNR being adjusted subsequently in an adaptive procedure (Brand \& Kollmeier, 2002) based on word scoring. The procedure aimed at finding the signal-to-noise ratio (SNR) that yielded a sentence recognition score of $50 \%$ correct, defined as the speech-reception-threshold (SRT). To address potential learning effects (Theelen-van den Hoek et al, 2014) and familiarize participants with the task, two training lists of 20 sentences were administered (one for each type of noise) prior to the start of actual testing. The results of these training lists were excluded from analysis. To obtain a reliable indication of directional benefit (Keidser et al, 2013) in each of the six test conditions, all conditions were assessed twice in a randomized order that was identical for test and retest. Each list consisted of 20 sentences and had a test time of 5 minutes on average. The sequence of lists was kept constant for all participants across randomized test conditions in order to prevent the usage of the same list twice within one participant. Overall this procedure resulted in 12 lists per participant (2 lists * 6 conditions). The actual speech-in-noise test had an average total duration of 60 minutes. To counteract fatigue, two intermissions were scheduled, one halfway through the speech-in-noise test and one before switching to the listening effort measurements. Additional breaks could be taken according to individual need.

When the adaptive procedure led to an invalid SRT outcome, defined as a SNR result outside the range of presented levels or above $15 \mathrm{~dB}$ SNR (Kaandorp et al, 2015), the outcome was omitted. If there were two valid outcomes, the final result per condition was calculated as the mean of test and retest.

\section{LISTENING EFFORT}

Participants were asked to rate the effort it took to listen to sentences in noise for the listening effort test (II). Rating was performed using a vertical scale with 13 discrete points (7 named categories interspersed by an empty category) ranging from 'no effort' (score 0) to 'extreme effort' (score 12) (Luts et al, 2010). The corpus of unique sentences from the Dutch Matrix test (Houben \& Dreschler, 2015) was also used for effort rating. Noise was presented at a fixed overall level of $65 \mathrm{~dB}$ SPL. In order to evaluate listening effort on top of speech intelligibility, the level of speech was set at the participant's individual SRT outcome in the speech intelligibility test for the corresponding condition. Listening effort was assessed at three levels: the participant's individual SRT, $5 \mathrm{~dB}$ above (SRT+5) and $10 \mathrm{~dB}$ above SRT (SRT+10). Every time a level was presented, one randomly selected sentence was repeated until the participant was confident enough to provide a rating. After a practice run with each level presented only once, every level was presented five times and the result was calculated as the mean of these five ratings. All six test 
conditions were assessed in random order with an average total test time of 12.5 minutes.

NOISE TYPE

Both outcome measures were assessed in two maskers: stationary (A) and fluctuating (B) noise. The default stationary noise associated with the Dutch Matrix (Houben et al, 2014) test was applied. This noise is composed of a randomlyaligned superposition of all the sentences in the test corpus and therefore has the same average power spectrum as the speech material (Houben et al, 2014). As fluctuating noise masker, a modification of the International Female Fluctuating Masker (IFFM) (Holube, 2011) was applied. The IFFM consists of a multilingual voice signal that has the spectral and temporal characteristics of a single speaker but is non-intelligible as a whole. The modification consisted of decreasing the fundamental frequency of the IFFM signal to male standards $(127 \mathrm{~Hz})$ to encompass extra information within the lower frequency range of aidable residual hearing in bimodal users. Both noises were checked on spectral comparability and scaled to the same root-mean-square (RMS) level.

\section{Set-up}

Testing was performed in a sound-attenuated booth using a desktop computer and the Oldenburg measurement applications (OMA) software package (HörTech gGmbH, Oldenburg, Germany). Participants used a touchscreen to self-administer all tests. Sounds were directed via analog lines of two externally connected Multiface II $^{\mathrm{TM}}$ soundcards (Hammerfall DSP System, RME, Audio AG, Haimhausen, Germany). The participant was seated amid an array of 6301B3X loudspeakers (Fostex, Tokyo, Japan) with a radius of one meter (Fig 3). Speech was presented in front $\left(0^{\circ}\right)$ while noise was continuously played from five surrounding speakers (+$\left.45^{\circ},+-90^{\circ}, 180^{\circ}\right)$. By applying a fixed randomly generated phase delay to the same basic signal in each of the five masker channels, the surrounding noise was emitted in an uncorrelated manner. This resulted in a diffuse interference field, representative of a challenging situation like a restaurant dinner. Each loudspeaker was first calibrated individually for the same presentation level. Afterwards an overall adjustment was applied to the five masker channels to reach the desired calibration level for the total noise field. 
FIGURE 3. Test set-up.

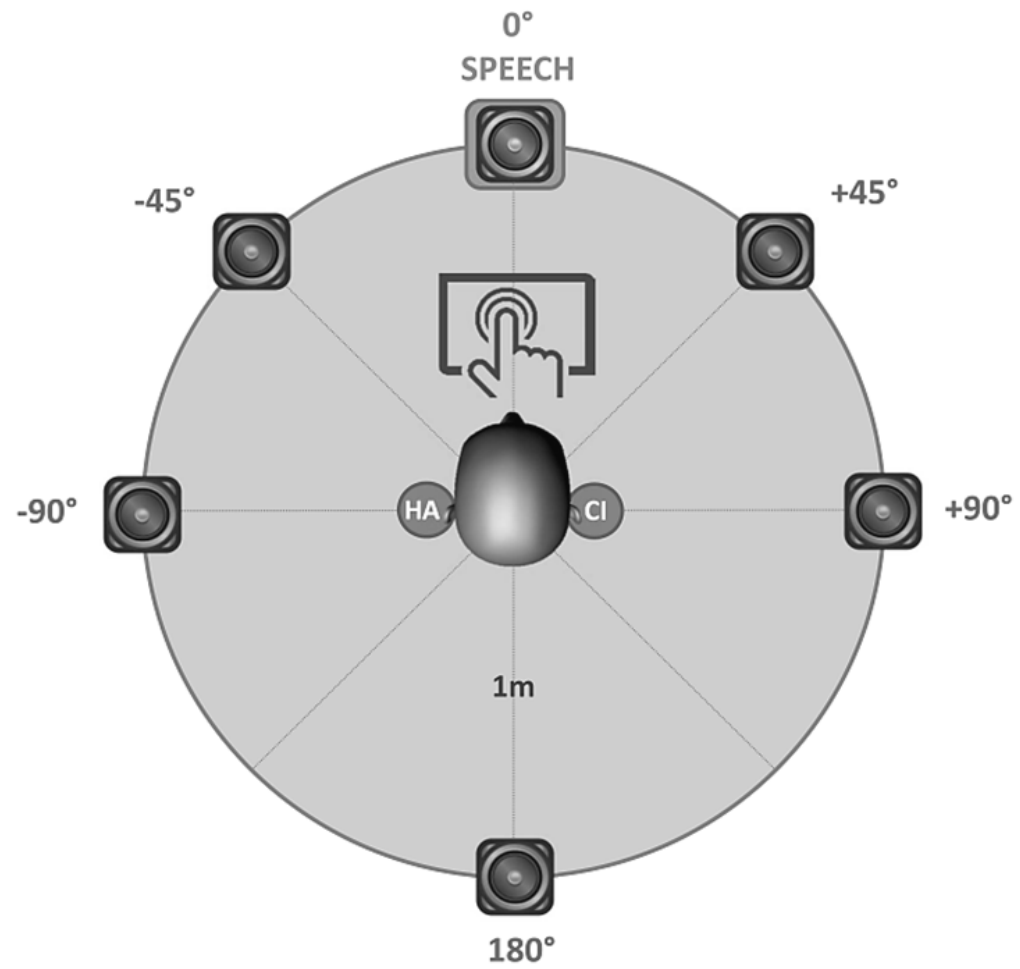

Six loudspeakers were positioned in a circle of $1 \mathrm{~m}$ around the participant, who was fitted with a cochlear implant (CI) and hearing aid (HA) in opposite ears. Speech was always presented in front $\left(0^{\circ}\right)$ while noise was simultaneously presented from the other five speaker locations. Participants used a touchscreen in front to self-administer all tests. Online available at: doi:10.1371/journal.pone.0160829.g003 


\section{SAMPLE SIZE CALCULATION}

Sample size estimation for the primary outcome of speech intelligibility was based on data available from literature. The monaural beamformer under investigation has been observed to provide an improvement of 5.2 up to $5.6 \mathrm{~dB}$ SNR with a standard deviation of 0.7 up to $1.7 \mathrm{~dB}$ SNR (Buechner et al, 2014; Geissler \& Arweiler, 2014) compared to an omnidirectional microphone (in or behind the pinna) in unilateral CI users. While the effect of asymmetry has not yet been investigated for beamforming in CI recipients, there are some studies in hearing aid users which have demonstrated a significant benefit of a symmetric over an asymmetric setting with an improvement of 1.9 up to $2.4 \mathrm{~dB}$ SNR (Hornsby \& Ricketts, 2007; Mackenzie \& Lutman, 2005; Ricketts \& Picou, 2013). To be able to detect the smallest primary effect, the estimated effect size within this study was set at 1.1 (i.e. $1.9 \mathrm{~dB} / 1.7 \mathrm{~dB}$ ). The required sample size was calculated for a paired samples statistical test using the statistical software G-Power 3.1.9 (Faul et al, 2007). The power analysis indicated that a sample of 11 (parametric) up to 12 (non-parametric) subjects was required to attain a power of $80 \%$. Based on these prospective calculations sample size was set at 12 .

\section{StatISTICAL ANALYSIS}

The outcome data were inspected for missing values. One-way random intraclass correlation coefficients (ICC) (Shrout \& Fleiss, 1979) were obtained to evaluate the reliability of the outcome measures. Normality was checked by the Shapiro-Wilk test and visual inspection of the outcome distributions using histograms and Q-Q plots.

A two-way repeated measures analysis of variance (ANOVA) was conducted to investigate the influence of two factors, namely directionality and noise type, on speech intelligibility. The factor directionality included three levels (omni, asymmetric, symmetric) and the factor noise type consisted of two levels (stationary, fluctuating). Listening effort ratings were compared across three factors by a three-way repeated measures ANOVA: the factors directionality and noise type were the same as for speech intelligibility outcomes while the third factor refers to three tested levels (SRT, SRT+5 or SRT+10). To correct against sphericity violations a Greenhouse-Geisser adjustment was applied. Where statistically significant effects were identified, post-hoc comparisons were performed with two-tailed paired samples t-tests. Mean pairwise differences are presented accompanied by the standard error (SE). An alpha value of 0.05 was considered with a Bonferroni adjustment for multiple comparisons. 


\section{RESULTS}

\section{SPEECH INTELLIGIBILITY}

Individual SRT outcomes for all bimodal participants are presented (S1 Table). No missing data were ascertained. Only one valid outcome could be obtained for one of the six tested conditions for six of the participants. For all the other test conditions and for all the other participants the mean of the two outcomes was taken. When both outcomes for test and retest were available, the ICC for the average outcome as well as for a single measure was found to lie between 0.72 and 0.95, indicating a substantial to almost perfect reliability (Landis \& Koch, 1977) of the outcome measure in all conditions.

A graphic presentation of the average bimodal SRT outcome across all test conditions is shown alongside the average results of a normal hearing reference group in both noise types (Fig 4). Data was normally distributed. In the bimodal study group, the two-way ANOVA indicated that both the main effects of directionality $\left[\mathrm{F}(1.40,15.38)=50.30, \mathrm{p}<0.001, \eta_{\mathrm{p}}{ }^{2}=0.82\right]$ and noise type $\left[\mathrm{F}(1.00,11.00)=237.13, \mathrm{p}<0.001, \eta_{\mathrm{p}}{ }^{2}=0.96\right]$ were highly significant. There appeared to be no interaction effect between directionality and noise type. Posthoc comparisons demonstrated speech intelligibility to be significantly better for the asymmetric setting compared to the omnidirectional reference with a difference of $1.57 \pm 0.20 \mathrm{~dB}$ SNR $(\mathrm{p}<0.001)$. Symmetric directionality provided an additional significant improvement of $1.07 \pm 0.23 \mathrm{~dB}$ SNR $(\mathrm{p}<0.001)$ compared to the asymmetric configuration. Overall this resulted in a significant difference of $2.64 \pm 0.34 \mathrm{~dB}$ SNR $(\mathrm{p}<0.001)$ between symmetric directionality and the omnidirectional reference.

The post-hoc comparison between noise types showed that SRT outcomes were significantly higher in fluctuating noise compared to stationary noise $(7.04 \pm 0.46 \mathrm{~dB}$ SNR $(\mathrm{p}<0.001))$. In the normal hearing reference group, however, speech intelligibility proved to be slightly better in fluctuating compared to stationary noise with a significant difference of $1.28 \pm 0.46 \mathrm{~dB}$ SNR $(\mathrm{p}=0.03)$. 
FIGURE 4. Speech intelligibility outcomes.

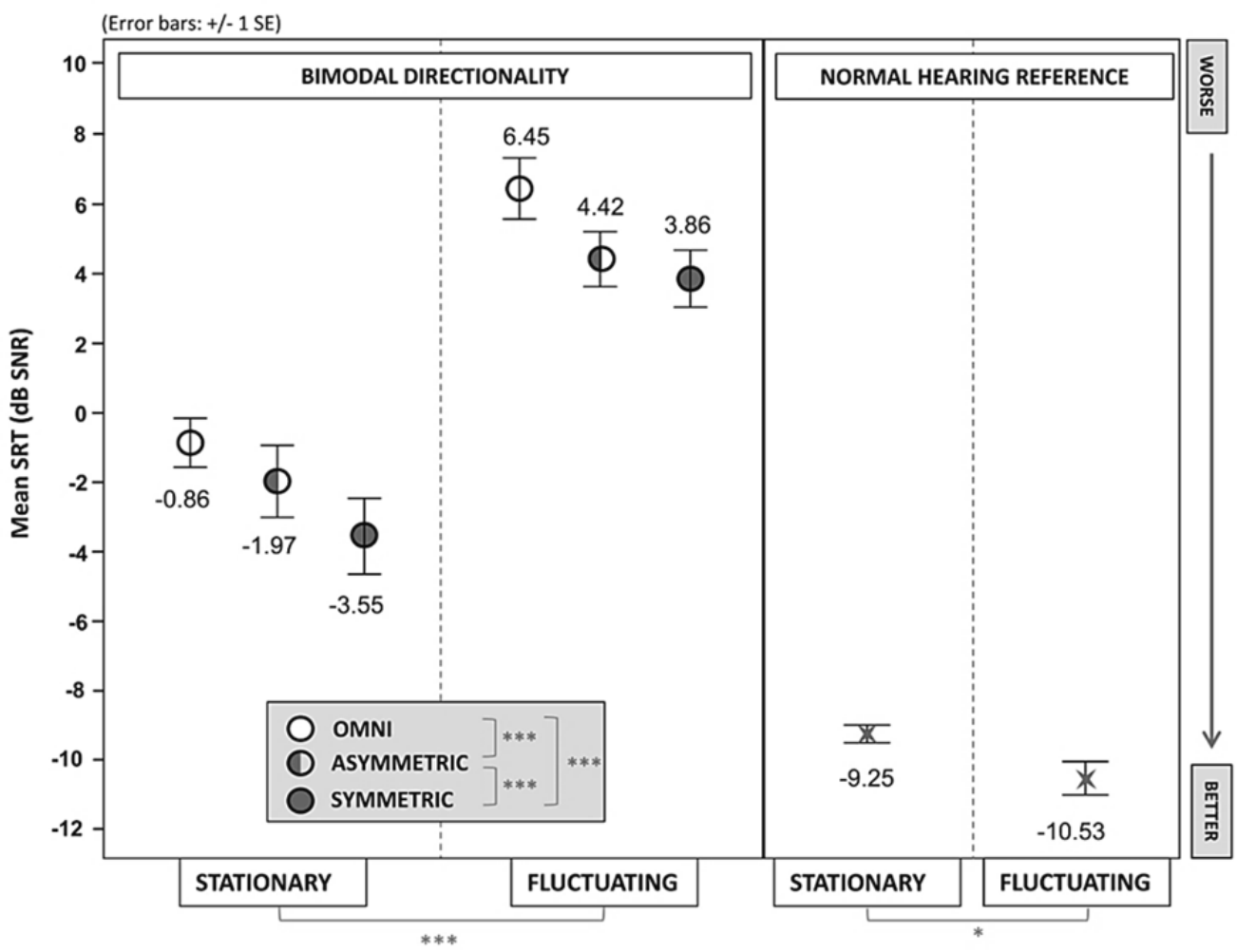

Mean speech reception thresholds (SRT) in noise of the bimodal study group are presented for the six tested conditions. Each condition consisted of a directionality configuration (omnidirectional, asymmetric, symmetric) assessed within two different types of noise (stationary, fluctuating). For comparison, mean speech intelligibility scores of a normal hearing reference group tested in the same set-up are shown. A lower SRT-value represents a better outcome. Significant differences between test conditions are flagged $\left(* \mathrm{p}<0.05,{ }^{* *} \mathrm{p}<0.01,{ }^{* * *} \mathrm{p}<0.001\right)$.

Online available at: doi:10.1371/journal.pone.0160829.g004

\section{LISTENING EFFORT}

Individual listening effort ratings for all bimodal participants are listed in S2 Table. No missing data occurred. The ICC for the average across five ratings was found to lie between 0.64 and 0.95 , indicating a substantial to almost perfect reliability (Shrout \& Fleiss, 1979) of the listening effort measurement in all conditions.

Mean ratings of bimodal listening effort across all test conditions are presented (Fig 5). Data was normally distributed. The three-way ANOVA showed no significant main effects. There was also no interaction between directionality and noise type and level; directionality and noise type; and directionality and level. There was an interaction between noise type and level $[F(1.53,16.88)=10.42$, $\left.\mathrm{p}=0.002, \eta_{\mathrm{p}}{ }^{2}=0.49\right]$. Therefore the simple effects of those two factors were 
further investigated. The results showed that listening effort was rated significantly higher at the participant's SRT ( $9.68 \pm 0.34$ points) when compared to SRT $+5(6.65 \pm 0.38$ point) and SRT +10 (4.22 \pm 0.39 points) in stationary noise (all $\mathrm{p}<0.001)$. The same order of level effect was observed in fluctuating noise comparing the rated effort at SRT ( $7.31 \pm 0.57$ points), SRT $+5(4.87 \pm 0.57$ points $)$ and SRT $+10(3.69 \pm 0.55$ points) (all $\mathrm{p}<0.002)$. Listening effort was assigned a significantly lower rate in fluctuating when compared to stationary noise at SRT (difference $2.38 \pm 0.55$ points, $\mathrm{p}=0.001$ ) and $\mathrm{SRT}+5$ (difference $1.78 \pm 0.56$ points, $\mathrm{p}=0.008$ ) but not at SRT +10 (difference $0.53 \pm 0.31$ points, $\mathrm{p}=0.11$ ).

FIGURE 5. Listening effort outcomes.

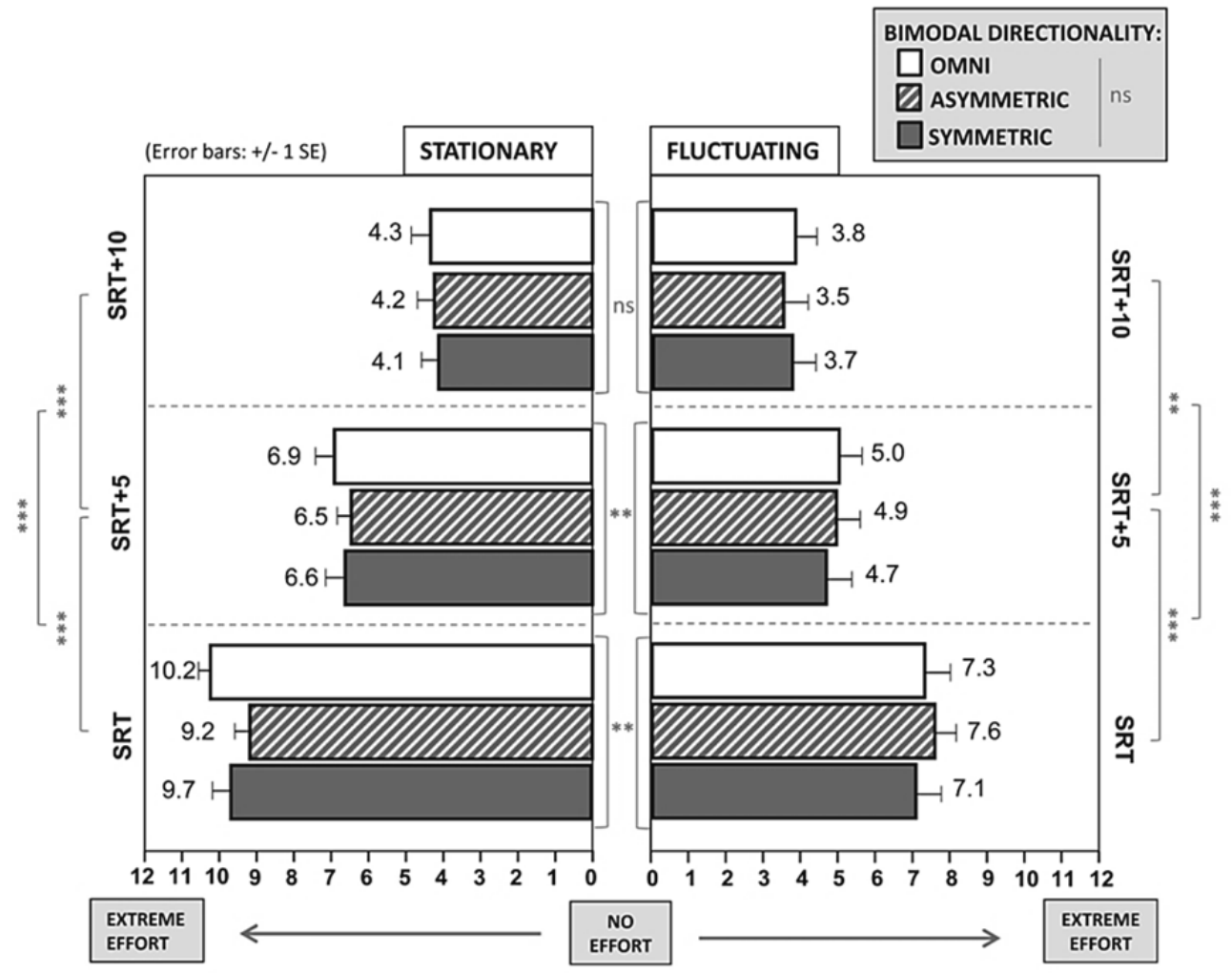

Mean Listening Effort (rating scale)

Mean listening effort ratings on a scale of 0 ('no effort ') to 12 ('extreme effort') for the bimodal study group are presented for six test conditions at three levels. Test conditions consisted of a directionality configuration (omnidirectional, asymmetric beamforming, symmetric beamforming) assessed for two different noise types (stationary, fluctuating). Tested levels of SRT, SRT +5 and SRT +10 are expressed as levels relative to the participant's individual speech-reception threshold (SRT) on the speech intelligibility task in the corresponding condition. Significant differences between test conditions are flagged $\left(* \mathrm{p}<0.05,{ }^{* *} \mathrm{p}<0.01,{ }^{* * *} \mathrm{p}<0.001\right)$. Ns $=$ not significant. Online available at: doi:10.1371/journal.pone.0160829.g005 


\section{DISCUSSION}

\section{SUMMARY OF FINDINGS}

Application of a monaural beamformer in bimodal CI-recipients improved speech intelligibility in stationary as well as fluctuating noise. Asymmetric directivity provided an average benefit of $1.6 \mathrm{~dB}$ compared to the omnidirectional standard. Symmetric directivity revealed an additional benefit of $1.1 \mathrm{~dB}$, leading to an overall improvement of $2.6 \mathrm{~dB}$. Listening effort decreased with increasing SNR but did not show an effect of directivity on top of speech intelligibility. Overall, bimodal users performed about $7 \mathrm{~dB}$ SNR worse in fluctuating as compared to stationary noise, while more listening ease was reported.

\section{SPEECH INTELLIGIBILITY}

\section{DEgREE OF DIRECTIONAL BENEFIT}

The effect of the investigated monaural beamformer has been previously reported to be 3.7 up to $5.6 \mathrm{~dB}$ in unilateral CI-recipients (Buechner et al, 2014; Geissler \& Arweiler, 2014). Compared to those findings, the unilateral degree of benefit in this study is rather small $(1.6 \mathrm{~dB})$ although significant. It is known that the benefit of directional systems is dependent on the used listening test set-up (Kompis \& Dillier, 2001). The current test set-up included speakers up to \pm 45 degrees, which still fell within the frontal beam of this monaural beamformer according to its polar plot (Buechner et al, 2014). The demanding spatial setting therefore is thought to be the primary reason for the found degree of directional benefit. To a smaller extent also the used reference condition could have played a role. Directivity in the CI-ear namely was compared to the reference of an in-the-concha microphone, already giving a first degree of natural directionality (FrohneBüchner et al, 2004) and leaving less room for improvement through beamforming (Geissler \& Arweiler, 2014; Gifford \& Revit, 2010). Withal, compared to results of unilateral CI-studies (Buechner et al, 2014; Geissler \& Arweiler, 2014), it should also be emphasized that this study evaluated bimodal listeners. Although the benefit of bimodal hearing was not separately assessed, it is known that bimodal hearing can provide significant bilateral and binaural benefits (Blamey et al, 2015; Büchner et al, 2009; Ching et al, 2007; Illg et al, 2014; Schafer et al, 2011; Sheffield \& Gifford, 2014). There is thus less room for improvement by beamforming algorithms when listening binaurally (Weissgerber et al, 2015), probably explaining the smaller degree of directional benefit found in this study. 


\section{EFFECT OF (A)SYMMETRIC DIRECTIVITY}

To date asymmetric directivity has only been suggested in bilateral HA-users as a permanent setting instead of manually switching between directional modes (Cord et al, 2011; Cord \& Walden, 2007). According to the "better SNR ear" principle, the effective SNR should be at least as good as the better of the two ears (Zurek, 1993). But the reported difference between asymmetric and symmetric directivity varies across HA-studies, ranging from no difference (Bentler \& Egge, 2004; Cord et al, 2011; Cord \& Walden, 2007; Kim \& Bryan, 2011) to a significant benefit in favor of the symmetric configuration (Hornsby \& Ricketts, 2007; Mackenzie \& Lutman, 2005; Ricketts \& Picou, 2013). However, CI-recipients are a different population, especially in the event of bimodal fitting. In bimodal users an asymmetric situation is often predetermined, having a CI in one ear and a HA in the other. The current study is the first to assess (a)symmetric directivity within a group of bimodal listeners. As demonstrated by the CNC scores in quiet (Table 1), the CI-ear was the primary speech input for most participants. Therefore only the CI was put in directional mode in the asymmetric setting. Results revealed a substantial improvement of the symmetric relative to the asymmetric configuration. Symmetric directivity $(2.6 \mathrm{~dB})$ almost doubled the advantage of asymmetric directivity (1.6 dB). Although no studies are known to evaluate the effect of (a) symmetric directivity within bimodal or bilateral CI-recipients, a recent study (Weissgerber et al, 2015) did point towards the benefit of symmetric directivity when comparing groups. They tested both bimodal and bilateral CI-users on speech intelligibility in noise with and without a monaural adaptive beamformer activated in their CI(s). The SRT improvement was found to be twice as high in the bilateral group as in the bimodal group, demonstrating the benefit of a bilateral combination of beamforming. A set-up with a moving noise source in the back hemisphere and the bilateral directivity benefit was linked to the effect of headshadow. However, the current study used a fixed set-up. It may therefore be that the effects of summation (combining two comparable inputs) and complementarity (combining two inputs with access to supplemental information (Kokkinakis \& Pak, 2014)) played a major role in the demonstrated symmetric benefit in this bimodal population.

\section{EFFECT OF NOISE TYPE}

In contrast to stationary noise, the fluctuating modified IFFM noise contained temporal gaps, and although non-intelligible, resembled a single speaker possibly inducing informational masking. A normal hearing reference group scored about $1.3 \mathrm{~dB}$ better in fluctuating when compared to stationary noise. By listening "in the noise gaps", normal hearing listeners are known to benefit from a masking release up to $7 \mathrm{~dB}$ (Francart et al, 2011). The degree of normal hearing masking release in 
this realistic set-up was smaller, probably because temporal fluctuations were reduced by presenting multiple uncorrelated spatially separated sources simultaneously. The average broad band modulation depth of the fluctuating masker (calculated according to IEC 60118-15) was $7 \mathrm{~dB}$ for the five-talker signal compared to $17 \mathrm{~dB}$ for the single talker signal. For reference, the modulation depth of the stationary noise was $2 \mathrm{~dB}$ albeit single or multi sourced.

In contrast to the normal hearing reference, bimodal listeners were not only unable to benefit from available gaps, they experienced a detrimental effect $(7 \mathrm{~dB})$ from the fluctuating masker when compared to stationary noise. This adverse effect, which has been previously reported for CI processing, is mainly caused by technical and physiological properties of CI stimulation (Zirn et al, 2016) resulting in a limited frequency and temporal resolution (Fu \& Nogaki, 2005; Nelson \& Jin, 2004; Qin \& Oxenham, 2003). Furthermore IFFM-like signals are known to have a distracting effect (Francart et al, 2011) which could result in a harder segregation task and thus demanding more attentional load (Rönnberg et al, 2013).

The noise reduction strategy available in the investigated CI processor could also have had an impact. This algorithm is known to improve speech intelligibility in stationary noise (Buechner et al, 2010; Kam et al, 2012) but has a smaller benefit in fluctuating noise (Advanced Bionics, 2012). This could have magnified the difference in SRT outcomes between the two noise types. The participants' daily setting of this algorithm was adopted in the test processor, resulting in different settings across participants. This between-subject factor was not included in the analysis due to the small sample size.

A study that compared a fixed and an adaptive directional microphone in CIpatients reported on average more directional benefit in fluctuating multi-talker babble noise as compared to a steady-state speech-weighted noise (Spriet et al, 2007). Concerning the benefit of directional microphones there might be an interaction with the masker type used to test the difference in speech recognition performance. The current study however cannot support this earlier finding, since no interaction between directionality and noise type was detected.

\section{LISTENING EFFORT}

\section{DIMENSION ON TOP OF SPEECH INTELLIGIBILITY}

Literature shows that listening effort is an additional dimension next to speech intelligibility and should be included when evaluating in noisy listening conditions (Klink et al, 2012b; Klink et al, 2012a). In the current study a quick and clinically applicable subjective rating task was included to assess listening effort 
independent of individual speech intelligibility (Schulte et al, 2008). Tested levels were therefore defined relative to the participant's SRT instead of conventionally testing at fixed SNRs. Given the slope of the optimized Matrix speech material in stationary noise for normal hearing subjects $(13.7 \% / \mathrm{dB})$ (Houben \& Dreschler, 2015), tested SRT levels are assumed to correspond to 50\% intelligibility (SRT) and go up to around $100 \%$ intelligibility for SRT+5 and SRT+10. It is known that the ease of listening increases with increasing SNR levels (Rudner et al, 2011; Schulte et al, 2008). Results did support this since effort was rated significantly lower at SRT+10 compared to SRT +5 and SRT for both noise types. But even at a level of SRT+10, the effort rated by bimodal participants did not drop to zero. This finding suggests that severe hearing impaired listeners may never report listening to be implicitly easy, supporting hypotheses linking hearing difficulties to an increased cognitive processing load (Advanced Bionics, 2012).

\section{EFFECT OF [A]SYMMETRIC DIRECTIVITY}

A reduction in effort through the application of directional fitting in HA-users has previously been reported in literature (Cord \& Walden, 2007; Wu et al, 2014). However by testing at fixed SNRs, earlier studies did not correct for speech intelligibility performance $(\mathrm{Wu}$ et $\mathrm{al}, 2014)$. A recent study in older HA listeners did report a reduction in effort in a dual task paradigm when using directional microphones at a fixed intelligibility level, even though no significant difference between objective and self-reported ratings of listening effort was found (Desjardins, 2016).

In the current bimodal study, levels relative to the participant's SRT were tested and no effect of directionality on the degree of listening effort was found. The observed directional benefit for speech intelligibility without additional effect on rated effort supports the statement that microphone directionality created an unadulterated SNR improvement. This result also demonstrated that the independent across ear operation of a monaural beamformer applied bilaterally did not seem to cause confounding cues.

\section{EFFECT OF NOISE TYPE}

Subjective effort rating has previously proven to be sensitive to differences in noise type (Hällgren et al, 2005). Results show that effort was rated lower in fluctuating noise than in stationary noise at SRT and SRT+5, but not at SRT+10. At SRT +10 , it could be expected that speech surpasses the noise and thus the basic effort of speech intelligibility is measured rather than noise influence. The effect of noise type on lower SNRs seems somewhat contra-intuitive since performance in fluctuating noise was significantly worse while effort was rated to be easier. Both 
speech intelligibility and listening effort are correlated with SNR, but they are known to be two different factors related in a non-linear manner (Klink et al, 2012 b) . The current results suggest that this relationship between intelligibility and listening effort is quite different for stationary and fluctuating noise. It seems that listening effort is closely related to the physically presented SNRs. Since levels were presented relative to the participant's SRT, SNR levels for fluctuating noise were up to $7 \mathrm{~dB}$ higher when compared to stationary noise, which was reflected in lower effort ratings. Furthermore also other unknown factors may have influenced this outcome. It has for example been suggested that the individual differences in working memory capacity may influence the relative perceived effort in different types of noise (Rudner et al, 2011). A study in young normal hearing adults showed that cognitive spare capacity performance was disrupted more in steadystate than in speech-like noise, possibly because selective attention could be used to ignore the speech-like background (Mishra et al, 2013). In general the found difference of noise type is supported by an earlier study in normal hearing, mild and moderately hearing impaired listeners that reported listening effort to be less in cafeteria noise compared to stationary noise, while intelligibility was greater in the latter (Schulte et al, 2007; Schulte et al, 2008).

\section{CHALLENGES AND FUTURE OUTLOOK}

Hearing aid manufacturers have been providing their devices with directional microphone systems for years. Likewise all of the major CI manufacturers implement a beamforming solution in their current speech processors. Therefore most bimodal recipients nowadays have access to a directional microphone system in both devices and thus comparable benefits as the ones observed in the current study are to be expected. However the long-term use of bimodal directivity in a variety of daily situations has not yet been addressed. Also the impact of the fact that most bimodal listeners make use of hearing systems giving access to different directional systems in both ears is still unknown. Even if the same beamforming system is available in both $\mathrm{CI}$ and HA, automatic program selection, which nowadays still operates independently per device, could cause the devices to select different settings at different scenes instead of a symmetric operation. The realtime and real-world application should therefore be the topic of further investigation and efforts should be made to enhance inter-device communication between $\mathrm{CI}$ and HA-systems. If such a bimodal communication system becomes available, also a binaural beamformer could be applied to even further improve directionality for bimodal listeners as has been proven for bilateral HA (Appleton \& König, 2014) and bilateral CI-recipients(Buechner et al, 2014). 


\section{APPENDIX}

S1 Table: Individual speech reception thresholds in noise (dB SNR).

Mean of two outcomes except ${ }^{1}$ based on single outcome when other outcome a outside range of presented SNR's or b larger than $15 \mathrm{~dB}$ SNR.

Available online: https://doi.org/10.1371/journal.pone.0160829.s001

\section{APPENDIX}

S2 Table: Individual listening effort ratings (scale 0-12).

Mean of five ratings on scale 0 (no effort) to 12 (extreme effort).

Available online: https://doi.org/10.1371/journal.pone.0160829.s002 


\section{ACKNOWLEDGMENTS}

We thank all participants for their time and effort during testing, Julie Bestel (Advanced Bionics) for her contribution during the protocol development and set-up preparations, and Michelene Chenault for her English editing services. Portions of these data were presented at the 12th European Symposium on Pediatric Cochlear Implants (June 2015, Toulouse, France), the 19th Jahrestagung of the Deutsche gesellschaft für Audiologie (March 2016, Hannover, Germany) and the British Cochlear Implant Group Meeting (April 2016, London, UK).

\section{DISCLOSURE OF INTEREST}

The authors of this manuscript have read the journal's policy and have the following conflicts: the work of the first author (EMJD) in this investigator-initiated study was financially supported by a research grant from Advanced Bionics Inc. The second author (AMLJ) provided statistical support, made possible by a grant from the Dutch Heinsius Houbolt foundation. The third author (JC) holds a scientific post in the Advanced Bionics European Research Center. For the remaining authors no conflicts were declared. The study was designed in close cooperation between MUMC+ and Advanced Bionics who also reviewed the final manuscript. Data collection, analysis and decision to publish were all solely accounted for by MUMC+. 



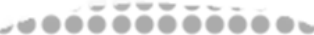

-

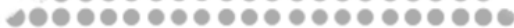

,

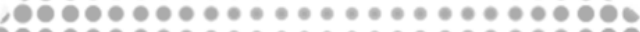

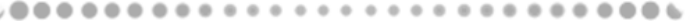

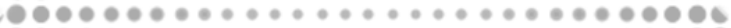

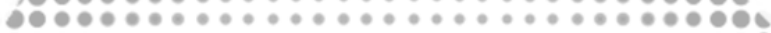

ر우

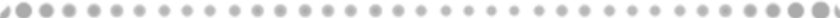

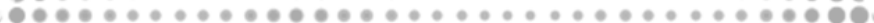

6.

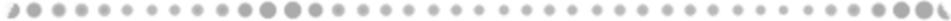

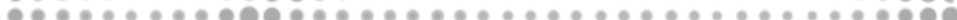

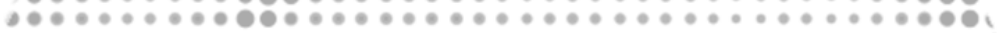

,

아웅ำ

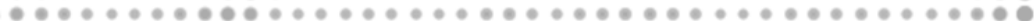

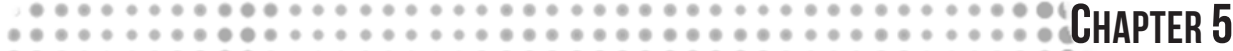

e

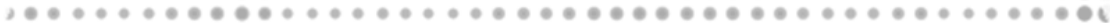

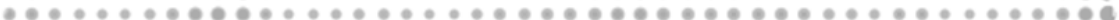

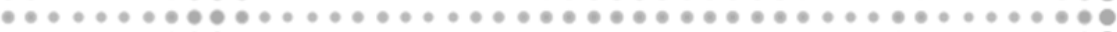

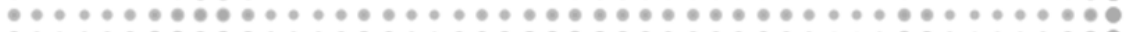

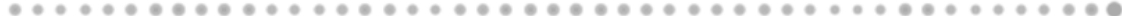

L.

: $: 2: \%$ REVISITING PLACE-PITCHMATCH IN

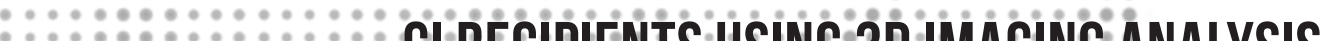
Cl RECIPIENTSUSING:3DIMAGING ANALYSIS

,

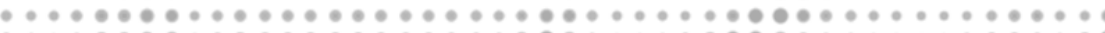

‥

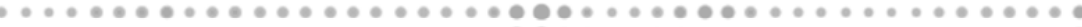

:

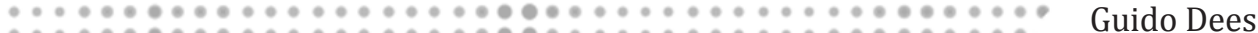

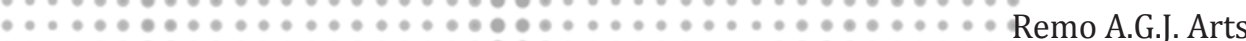

$+0$

(5)

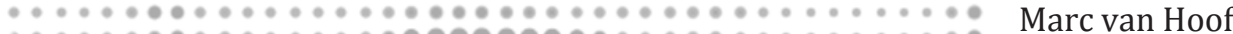

(

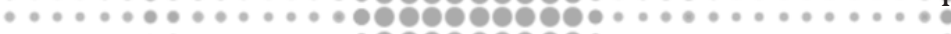

e.

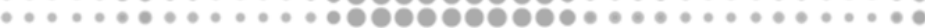

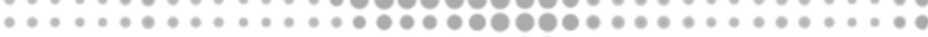

…*.............

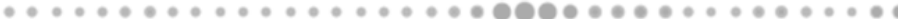

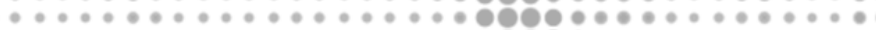

.... .............

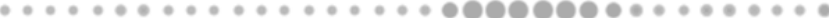

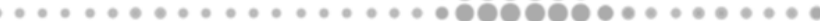

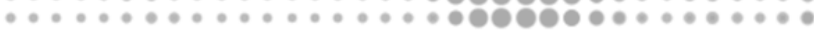

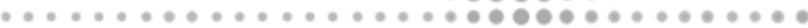

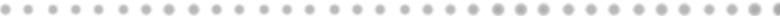

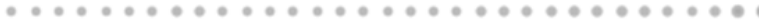

…

…

(3)

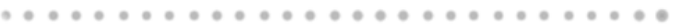

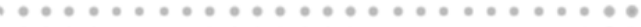

- . -

Annals of Otology, Rhinology \& Laryngology, 2016, Vol. 125(5) 378- 84

DOI: $10.1177 / 0003489415616130$ 
The objective of this study was to improve the estimation of the perceived pitch in a cochlear implant (CI) listener by using accurate 3-dimensional (3D) image analysis of the cochlear electrode positions together with the predicted tonotopical function for humans. A single-sided deaf CI user underwent a Cone-Beam computed tomography (CBCT) scan. Electrode contacts were marked in 3D space in relation to the nearest point on the cochlear lateral wall. Distance to the base of the lateral wall was calculated and plotted against the place-pitch function for humans. An adaptive procedure was used to elicit the perceived pitch of electrically evoked stimulation by matching it with a contralateral acoustic pitch. Results showed that the electrically evoked pitch percept matched well with the calculated frequency. The median mismatch in octaves was 0.12 for our method in comparison to 0.69 using the conventional Stenvers view. The method shows the potential of 3D imaging in CI fitting optimization. 


\section{INTRODUCTION}

The position of cochlear implant (CI) electrodes is important for the perception of pitch. Tonotopic organization, which relates place to pitch inside the cochlea and extends to the cortex, has shown to be of great importance in auditory perception (Oxenham et al, 2004). Using empirical evidence, Greenwood (1990) has quantified this map for humans and other species.

For CI users, it is to be expected that in a similar manner, electrical stimulation at any equivalent point in the cochlea would elicit a corresponding pitch. Yet, early studies only found limited proof of this place-pitch correlation in CI recipients: pitch-matching results were generally situated 1 to 2 octaves below calculated pitch estimations (Baumann \& Nobbe, 2006; Boëx et al, 2006; Dorman et al, 2007) and showed large variability especially beyond the first cochlear turn (Kalkman et al, 2014; Schatzer et al, 2014). In an attempt to explain this discrepancy, several theories are suggested in literature: methodological issues with the pitch-matching procedure (Carlyon et al, 2010a; Carlyon et al, 2010b; Green et al, 2012), tonotopical reorganization after CI experience (Reiss et al, 2014a; Vermeire et al, 2015), the site of electrical stimulation (Kalkman et al, 2014; Schatzer et al, 2014; Stakhovskaya et al, 2007), the degree of contralateral residual hearing (Vermeire et al, 2008), and cross-turn stimulation (Boëx et al, 2006; Frijns et al, 2001; Kalkman et al, 2014).

In the current study, it is hypothesized that measurement inaccuracy could have played a role in previous mismatching results. The conventional use of Stenvers view (Xu et al, 2000) to calculate imaging-based pitch predictions could have led to inaccurate results due to a variable (Todd \& Ball, 2004) and skewed perspective and thus an inaccurate estimation of (individual) electrode insertion angles, especially toward the apical end of the cochlea.

Here the traditional method is compared to a new method that combines highresolution 3-dimensional (3D) Cone-Beam computed tomography (CBCT) with a freely availably software package for image analysis in order to calculate the predicted pitch. This method is thought to be practically insensitive to artefacts introduced by the chosen reconstruction plane in CT or the perspective taken in an $\mathrm{X}$-ray. This imaging-based place-pitch estimation is validated in a single-sided deaf (SSD) subject using electrical stimulation in the cochlea while adaptively comparing it to the perceived pitch of an acoustic stimulus in the contralateral ear. 


\section{Materials AND MethodS}

SUBJECT

A 52-year-old female with an 11-year history of unilateral idiopathic progressive sensorineural hearing loss (92 dB hearing level [HL] averaged across 0.5, 1, and $2 \mathrm{kHz}$ ) and associated tinnitus, received a cochlear implant in the left ear as part of a prospective clinical trial (Arts et al, 2015). The trial was approved by the local Medical Ethical Committee (Maastricht University Medical Center) and was conducted in accordance with the Declaration of Helsinki. The subject was implanted with a FLEX28 ${ }^{\mathrm{TM}}$ electrode array (MED-EL Corporation, Innsbruck, Austria) using a round window approach. At the time of pitch matching, she had 18 months of experience with the OPUS 2 processor. In the contralateral acoustic hearing ear, a mild sensorineural hearing loss of $30 \mathrm{~dB}$ HL (averaged across $0.5,1$, and $2 \mathrm{kHz}$ ) was present.

\section{CONE-BEAMCT}

At 6 months post implantation, a CBCT examination was performed on clinical indication. The aim was to rule out postoperative migration in relation to the reported percept of manipulation-induced tinnitus. A 17-19 I-CAT ${ }^{\text {TM }}$ device (Imaging Sciences International, Hatfield, Pennsylvania, USA) with a tube current of $37.07 \mathrm{mAs}$ and a tube voltage of $120 \mathrm{kV}$ was used. One full rotation took 26.9 seconds. The CBCT scan resulted in a radiation exposure of approximately 0.05 $\mathrm{mSv}$. Raw data projection images were reconstructed using the I-CAT vision application (Imaging Sciences International) with an isometric voxel size of 0.2 $\mathrm{mm}$. No evidence of migration was found, and the tinnitus related to manipulation had already subsided upon performing the current experiment. Consequently, obtained imaging data could be used for additional pitch calculation analysis.

\section{Image Analysis and Pitch Calculation}

Image analysis and visualization was performed using the open source 3D Slicer 4 package (available for download at www.slicer.org) (Pieper et al, 2006). All electrode contacts were manually identified in 3 dimensions. The lateral wall and medial wall were delineated by a set of 93 and 54 fiducials at a height corresponding to the basilar membrane in 3 dimensions. The center of the modiolus was depicted by a line connecting the modiolus at the base and the apex of the cochlea. The Greenwood function with human reference values 2 was used to compute the electrode pitch by automatically choosing the nearest point on the lateral wall and entering the insertion depth relative to the subject's specific 
calculated cochlear duct length (Alexiades et al, 2015). For comparison purposes, the conventional cochlear Stenvers view (Xu et al, 2000) was reconstructed using a maximum intensity projection. As described by Xu et al. (2000) the predicted pitch was calculated by measuring the insertion angle of the individual electrode, relating it to the fractional length along the organ of Corti (Bredberg \& Bredrerg, 1968), and applying the standard Greenwood function for an average human cochlea (Greenwood, 1990). All custom algorithms and descriptive statistics were executed in Wolfram Mathematica version 10.0 using built-in functions (Wolfram Research Inc, Champaign, Illinois, USA).

\section{Electric Stimulation}

The OPUS2 processor was programmed to provide intracochlear electrical stimulation with looped patterns, independent of environmental sounds (Arts et al, 2015). Charge-balanced, cathodic first, biphasic pulses were continuously generated in monopolar mode with a phase duration of $37 \mu$ s and a stimulation rate of 1500 pulses per second (pps). Because place-coding of pitch is the primary interest of the current study, a constant stimulation rate of 1500 pps was used as to avoid potential confounding temporal effects of stimulation rate (Kong \& Carlyon, 2010).

During the place-pitch matching procedure, continuous electrical stimulation was presented on the single specific electrode (EL) to be tested. Stimulation was perceived to be comfortably loud as scored 6 on a numeric rating scale from 0 (inaudible) to 10 (extremely loud). To prevent loudness adaptation, stimulation was interrupted between test conditions.

Trading of between accuracy and practical time constraints, 4 electrodes with an estimated pitch perception within the frequency range of a standard audiometer (250- $8000 \mathrm{~Hz}$ ) were selected. Three apical electrodes (EL 1, 3, and 4) and 1 more basal electrode (EL 8) were each tested twice (test-retest: ordered EL 1-4-8-3-1-48-3).

\section{Pitch-Matching Procedure}

Pitch matching was performed using headphones (TDH-39P, Telephonics, Farmingdale, New York, USA) on the contralateral acoustic hearing ear. A pure tone or a one-third octave narrow band noise was presented, depending on which acoustic stimulus best resembled the perception of electric stimulation. According to the rate setting of the pattern generator (1500 pps), electric stimulation was first balanced in loudness with a $1500 \mathrm{~Hz}$ acoustic stimulus, using a 2-down and 1up adaptive staircase rule with a step size of $5 \mathrm{~dB}$ (Leek, 2001). Afterward, pitch 
matching was performed within the frequency range of $250 \mathrm{~Hz}$ up to $8000 \mathrm{~Hz}$, using a 3-alternative forced choice method. Both octave and interoctave frequencies were presented in separate "runs" from low-to-high and high-to-low frequencies. This way, a large range of starting frequencies was used in order to prevent octave confusion (Carlyon et al, 2010c). Subsequently, pitch matching was finetuned using semitone steps. Finally, precise loudness matching was repeated with a step size of $1 \mathrm{~dB}$ at the matched frequency. As a result, the final loudness balanced pitch matched frequency was found.

\section{RESULTS}

\section{CONE-BEam CT ANALYSIS}

When the CBCT is reconstructed to a Stenvers view, the individual electrodes can easily be identified, but the 3D orientation of electrodes in relationship to intracochlear structures is lost (Figure 1). The 3D reconstruction (Figure 2A) on the other hand does allow for a qualitative assessment that is independent of the perspective. Quantitative measurements are also possible (as plotted in Figure 2B). The cochlear duct length is calculated to be $37.2 \mathrm{~mm}$, and the most basal electrode is found to be either at the round window or just extracochlear. As the cochlea changes in inclination and curvature, large differences in the distance between the electrodes and the lateral and medial wall of the cochlea appear to exist.

FIGURE 1. Stenvers view reconstruction of the partial CBCT volume using a maximum intensity projection

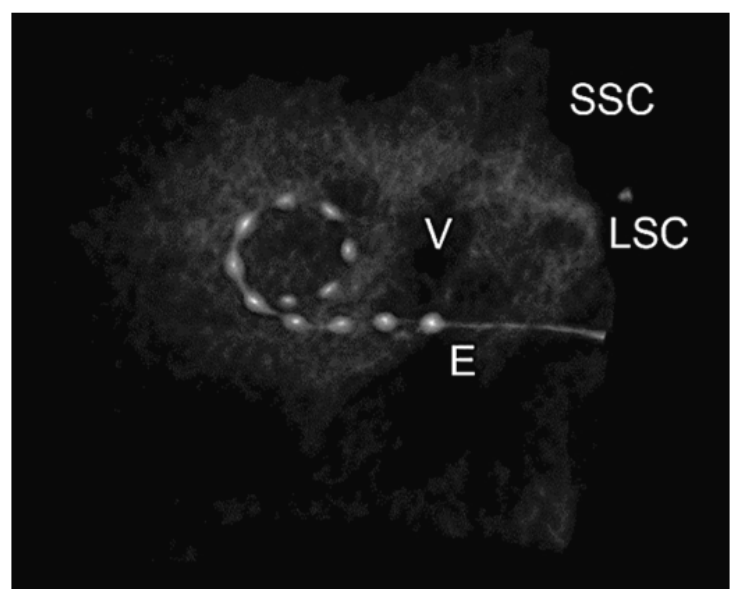

CBCT, Cone-Beam computed tomography; E, electrode array; LSC, lateral semicircular canal; SSC, superior semicircle canal; $\mathrm{V}$, vestibule. 
FIGURE 2. CI Electrodes in 3D in relation to the cochlea.
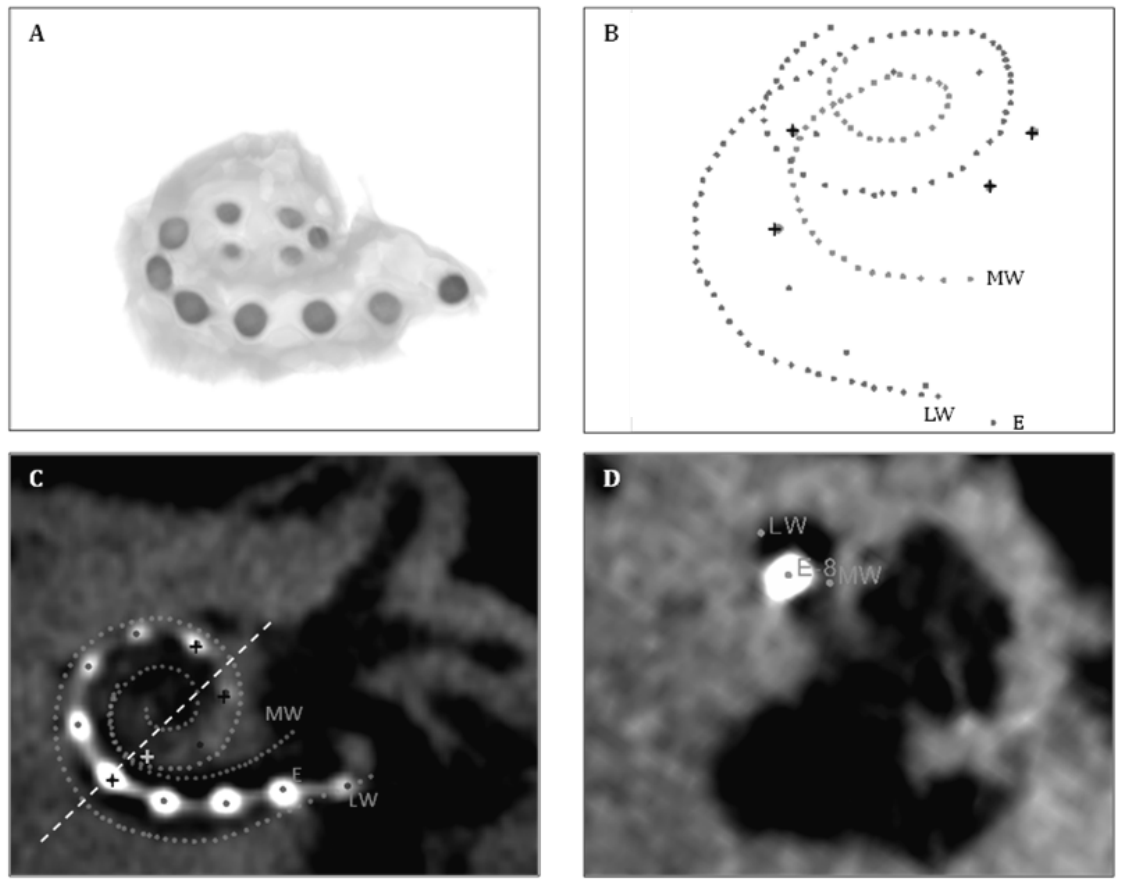

(A) Cone-Beam computed tomography (CBCT) 3D reconstruction of the cochlea. Electrodes are highlighted. (B) Detailed measurements of lateral wall (LW), medial wall (MW), and electrode contacts (E) allow for an accurate assessment of electrode position in relation to intracochlear structures. Crosses represent pitch-matched electrodes. (C) Overview of all markers in one CBCT slice with the white line delineating the cross section resulting in Figure D as a presentation of the individual pitch-matched electrode 8 and the nearest LW and MW markers.

\section{Place-Pitch Matching}

During place-pitch matching, the subject reported acoustic pure tones to best resemble the perception of electric stimulation. The median difference between matched frequencies for test and retest was found to be -0.09 octaves. The mean of test and retest was therefore considered to be the final result of the pitchmatching procedure. Results of electrodes 1, 3, 4, and 8 along with the calculated frequencies are presented in Table 1 and Figure 3. Mismatch is defined as the difference between the result of the pitch matching procedure and the frequency estimation calculated by each method. Pitch calculations based on the Stenvers reconstruction showed a median mismatch of 0.69 octaves (range, 0.14 to 0.98 ) compared to a mismatch of 0.12 octaves (range, -0.61 to 0.53 ) using the 3D analysis. 


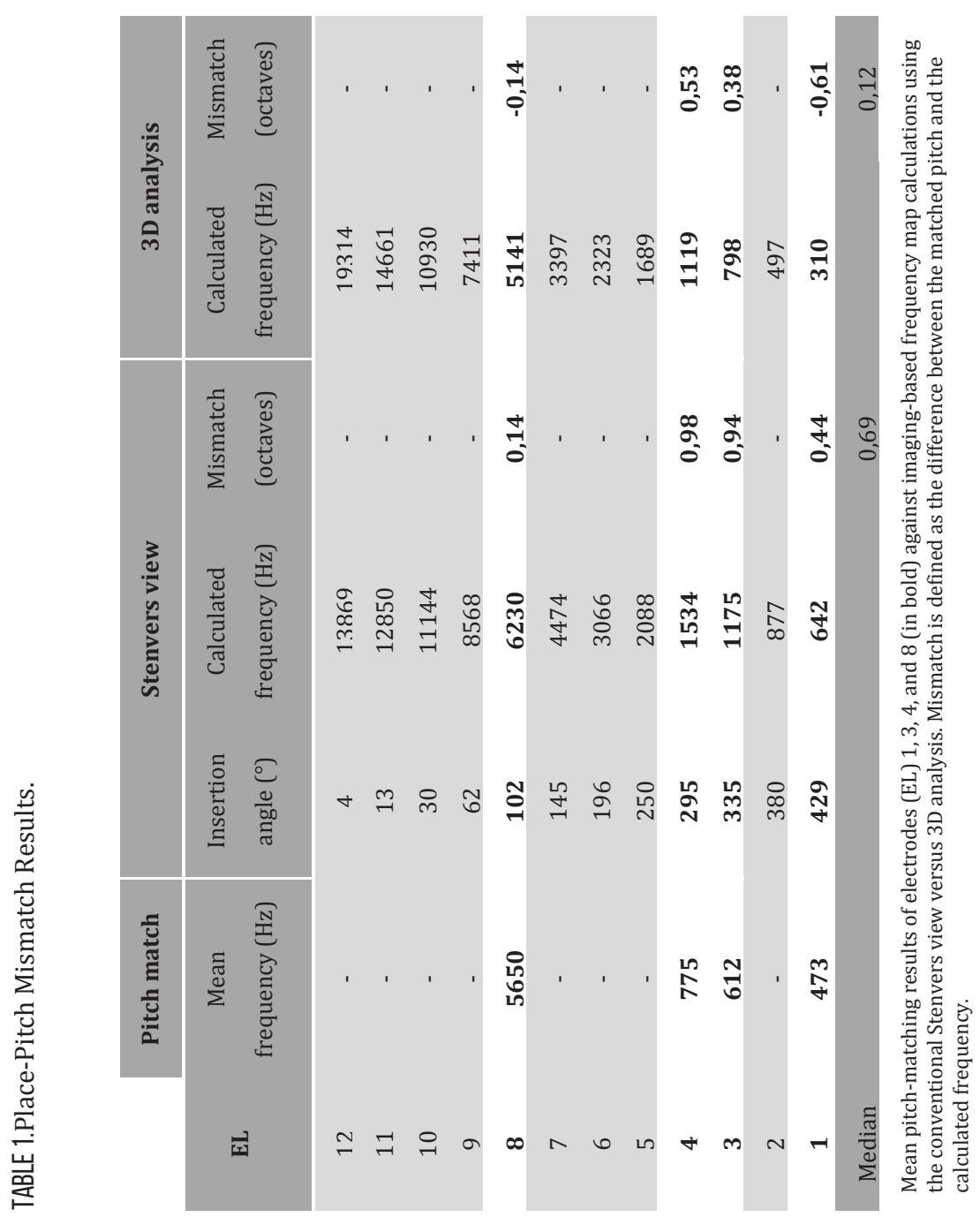


FIGURE 3.

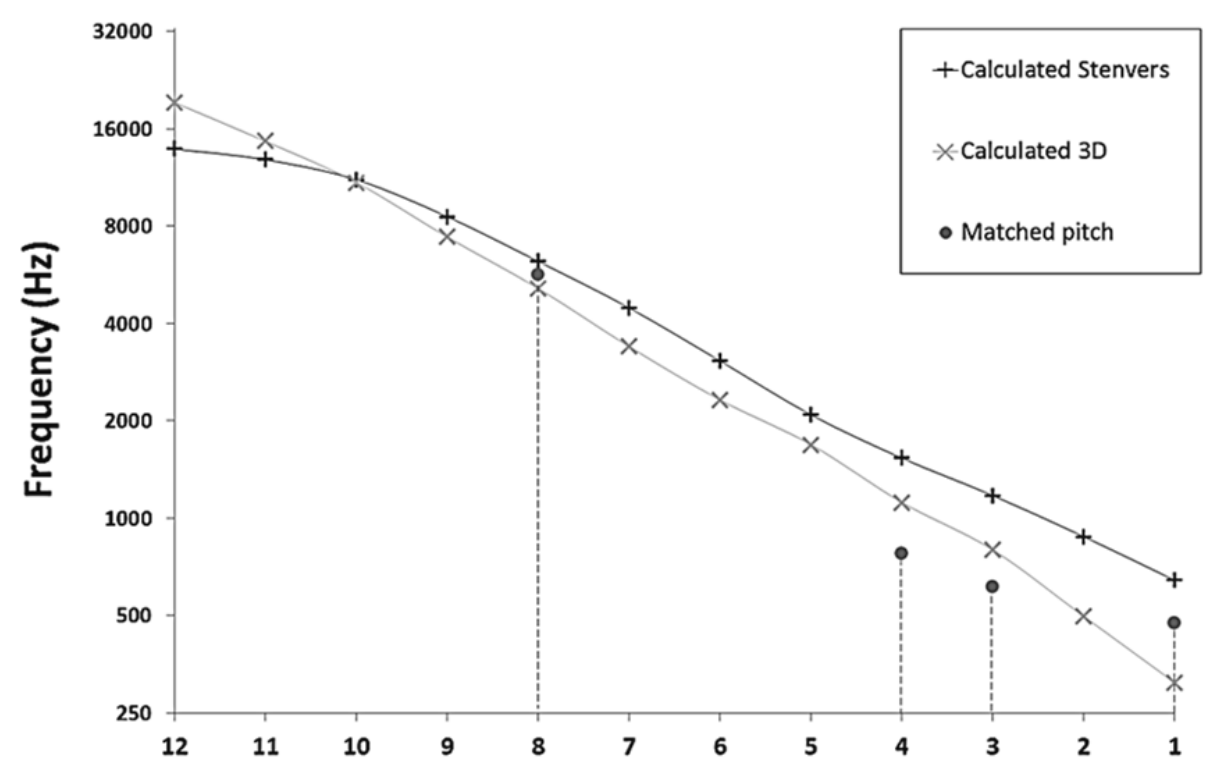

Electrode number

Electrode-pitch results as perceived by the subject (circles) are plotted against the calculated frequency map based on Stenvers view (straight crosses) and the newly proposed 3D method (oblique crosses). Dashed lines indicate the four pitch matched electrodes $1,3,4$, and 8 .

\section{DISCUSSION}

\section{INTERPRETATION OF RESULTS}

The Stenvers view (Xu et al, 2000) represents the traditional method of postimplant imaging and electrode-to-pitch mapping. Different from previous studies (Baumann \& Nobbe, 2006; Boëx et al, 2006; Dorman et al, 2007), the matched pitch in this study fell on average less than 1 octave below the frequency as calculated by the conventional method. However, here the Stenvers view was reconstructed using a 3D image, which is not in line with common practice. In clinical radiological practice, a higher variability and less optimal result is to be expected since a correct orientation of the subject is crucial. Nevertheless, the newly introduced method based on 3D measurements using CBCT imaging still achieved an even better approximation of the pitch-matching outcome, especially in the apical region. Now, the pitch percept of electrically evoked stimulation over the frequency range relevant for daily life functioning reasonably agreed with the frequency as calculated by the Greenwood function (Greenwood, 1990). 
While some recent pitch-matching studies also managed to find a place-pitch mismatch smaller than 1 octave (Vermeire et al, 2015; Vermeire et al, 2008), the conventional CT used in those studies is still inherently sensitive to the used reconstruction plane that can decrease its reliability. The new method on the other hand expresses the position of every individual electrode in relation to the closest point on the lateral wall, which in turn is expressed as an individual proportional length in order to implement the Greenwood function (Greenwood, 1990). This method ensures that position determination of the electrode or the lateral wall is only minimally affected by the viewpoint perspective. Moreover, no assumptions are made on the inclination or anatomy of the cochlea. Only the distance is accumulated over the course of the lateral wall and thus along the cochlear duct. Other notable attempts, including accurate 3D imaging augmented by models of the cochlea, have been proposed (Noble et al, 2014). However, those strategies did not yet attempt to address frequency mismatches in clinical CI users (Ali et al, 2015).

\section{Clinical and Audiological Relevance}

The (human) brain is known to be an excellent, adaptive, and flexible entity in analyzing and combining sound information (Litovsky, 2015). In order to achieve a high performance on speech discrimination (Siciliano et al, 2010; Zhou et al, 2010), music appreciation (Peretz et al, 2002), and sound source localization (Goupell et al, 2013), a natural and accurate perception of pitch in both ears is indispensable. So for the severe hearing impaired, who have regained the ability to hear by means of a cochlear implant (Fitzgerald et al, 2013), and even more for those who combine electric and acoustic hearing (eg. single-sided deaf subjects (Vermeire et al, 2008), users of electro-acoustic stimulation (Turner, 2008), and/or bimodal listeners (Guérit et al, 2014)), it is essential to pursue the "gold standard" of normal hearing if one aims to achieve the best possible outcome. It should thus be a primary requirement that electrical stimulation and the information it carries matches as closely as possible to what the human brain has evolved to cope with or learned to process instead of relying on plasticity to adapt to an induced mismatch (Reiss et al, 2014b; Reiss et al, 2014a; Vermeire et al, 2015). This ultimate goal can only be achieved by making use of high precision knowledge of intracochlear electrode positioning.

\section{FUTUREIMPROVEMENTS}

Further reducing individual electrode mismatches could be achieved by improving the presented method. The distance to the lateral and medial wall could be incorporated as illustrated in Figure 4. This way, information about the distance 
between electrodes and other surrounding intracochlear structures could be incorporated. Furthermore, taking into account the excitatory current spread would help to localize the area of stimulated nerve cells, for example in relationship to the spiral ganglion trajectory (Stakhovskaya et al, 2007). One then can estimate not only the center frequency but also establish the lower and upper frequency boundary of the corresponding electrode in the CI filter bank. Also, the conventional pitch-matching procedure needs optimization since the adaptive time-consuming procedure now still precludes one from testing every individual electrode in a clinical setting.

FIGURE 4.

A

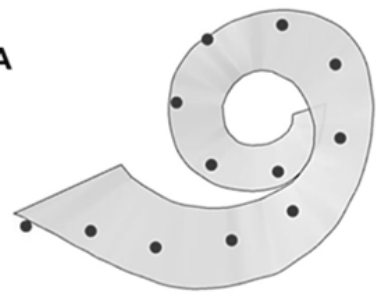

C

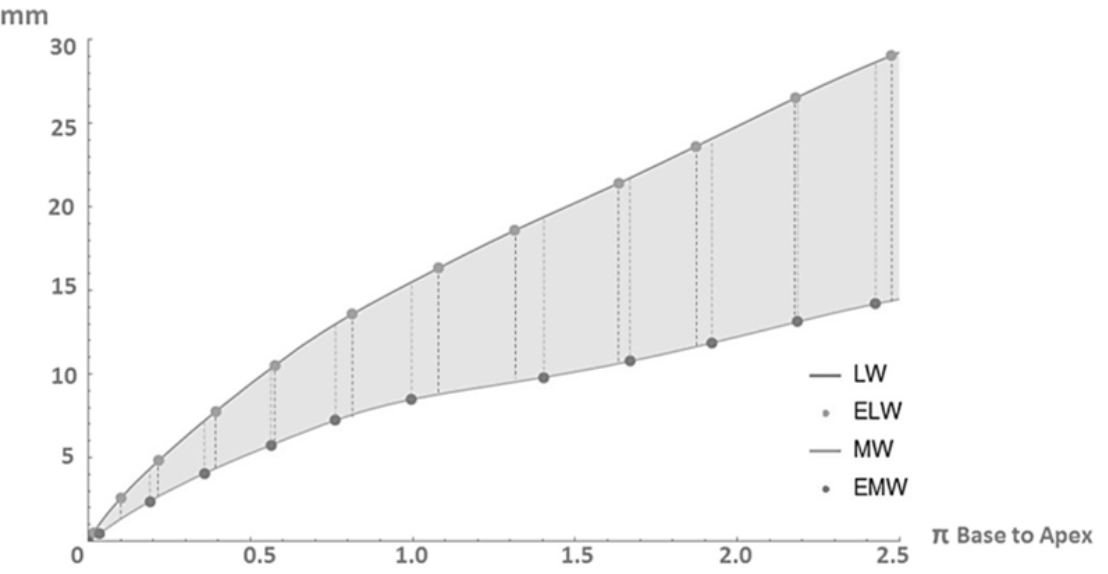

B

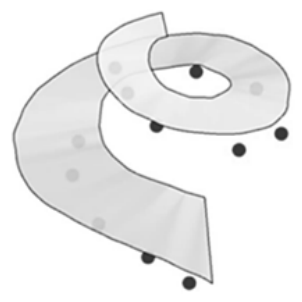

Intracochlear electrode positions relative to the medial (MW) and lateral wall (LW) in (A) inferior and (B) lateral view. (C) Electrode insertion depth (in mm) in relation to the nearest point on the LW (ELW) and MW (EMW) (from base to apex in radians). Dashed lines delineate the respective mismatches in insertion depth if measured only in relation to the LW or the MW, respectively. 


\section{LIMITATIONS}

This proof-of-principle investigation aimed at a high accuracy instead of a large sample size. The methods presented here should be further evaluated in a large clinical trial to estimate the variance in a larger sample size featuring different patient subgroups (eg. single-sided deaf subjects, bimodal and bilateral users). As these methods are within the reach of other researchers who have access to pitchmatching data, this short communication intends to bring awareness to this new alternative method. Another factor to take into account is the potentially induced tonotopic reorganization (Vermeire et al, 2015). Reorganisation can be expected to commence at $\mathrm{CI}$ activation due to an expected mismatch with the default $\mathrm{CI}$ frequency allocation table (Landsberger et al, 2015) and thus can only be accounted for in users upon first $\mathrm{CI}$ activation. Furthermore, the effect of stimulation rate should be addressed in more detail. In the current study, stimulation was kept constant at a frequently used rate of 1500 pps since this rate is well beyond the limit of temporal pitch cues (Fearn et al, 1999; Kong \& Carlyon, 2010). However, it is known that stimulation rate can alter the perceived frequency percept (Schatzer et al, 2014).

\section{CONCLUSION}

A new method that appears to reach a more accurate estimation of the perceived pitch at an associated cochlear electrode position is demonstrated in a CI-user with a single-sided deafness. This method based on 3D imaging could possibly be used to optimize CI fittings. 


\section{ACKNOWLEDGMENTS}

The authors would like to thank the researchers and developers of the different open source software packages that were used in this study and the subject for her intensive dedication during data collection.

\section{DISCLOSURE OF INTEREST}

The author(s) declared no potential conflicts of interest with respect to the research, authorship, and/or publication of this article. The author(s) received no financial support for the research, authorship, and/or publication of this article 


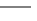




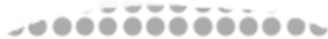

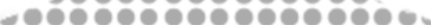

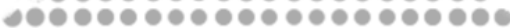

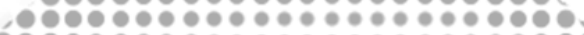

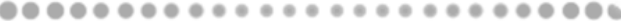

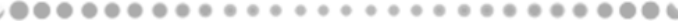

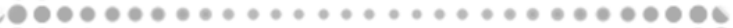





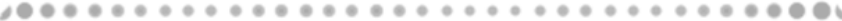

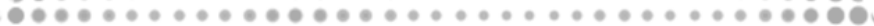

ค.

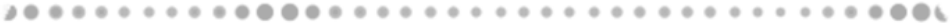

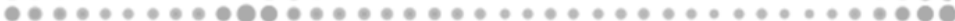

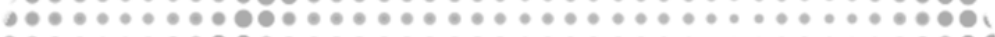

,

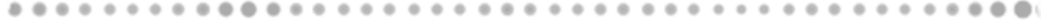

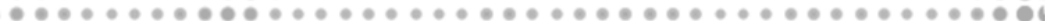

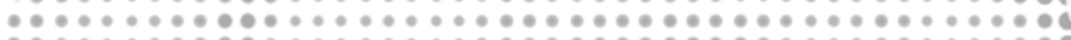

․‥

,

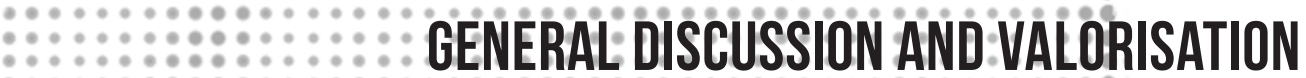

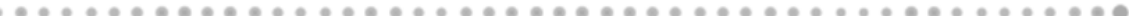

b

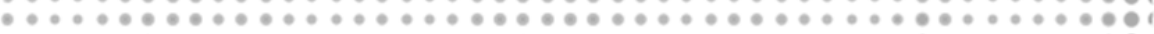

‥

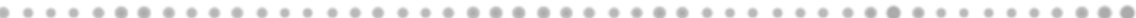

…

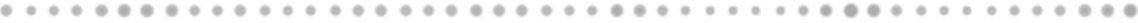

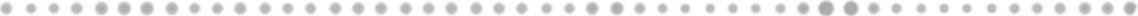

․

,

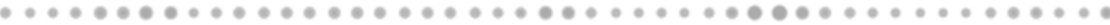

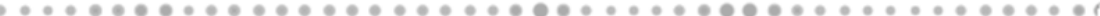

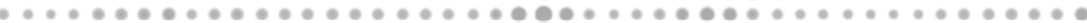

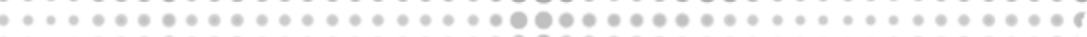

, ․ำ

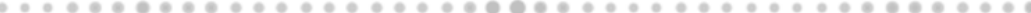

, +

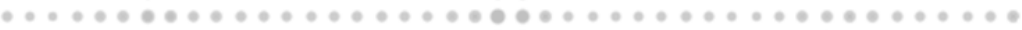

,

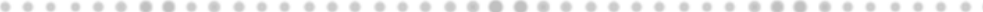

,

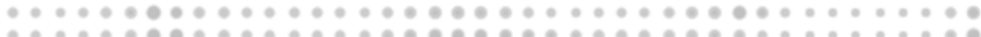

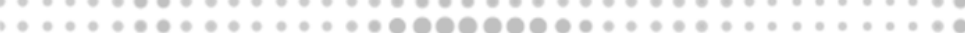

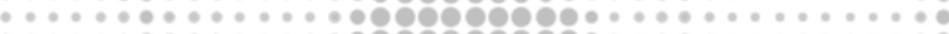

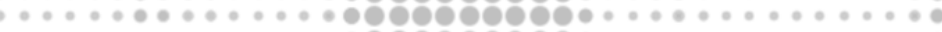

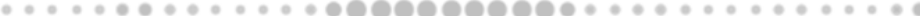

* * * * * *.....

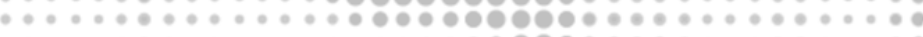

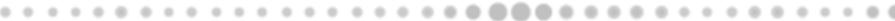

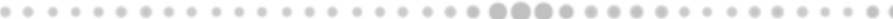

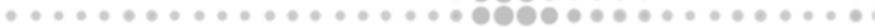

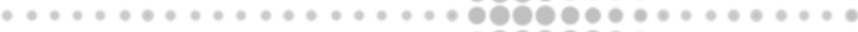

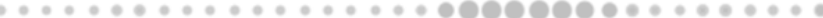

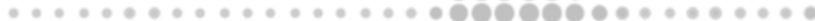

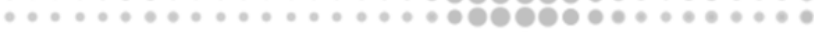

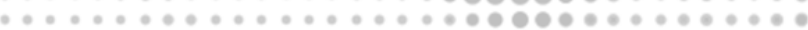

"น

․

:

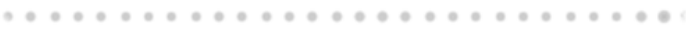

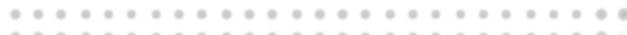

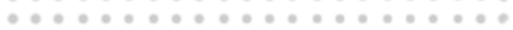

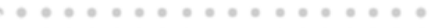





\section{THE RELEVANT NUMBERS}

The global number of hearing impaired people keeps rising. Today more than $5 \%$ of the world population (circa 460 million people) has disabling hearing loss ( $>40 \mathrm{~dB}$ HL in adults, $>30 \mathrm{~dB}$ HL in children) and this number is predicted to go up to about one in every ten people by 2050 (World Health Organisation, 2018). Contributing factors are thought to be aging, overall noise exposure and awareness of the adverse effects of hearing loss. Hearing loss namely is not only associated with incapacitating social restrictions but also has a significant cost on our economic society (Olusanya et al, 2014).

In 1995 a national UK study by the MRC Institute of Hearing Research showed that about $0.7 \%$ of the adult population (18-80 years old) had a severe hearing loss (70-94 dB HL) and 0.2\% had a profound hearing loss ( > $95 \mathrm{~dB}$ HL) (Davis, 1995). Based on these figures the number of eligible candidates for cochlear implantation (CI) may be estimated around $0.5 \%$, that is more than 30 million people worldwide. Today however, only a fraction of these people actually receive a CI. By the end of 2012 the total number of registered CI's worldwide was approximately 324,200 (NIDCD, 2016). In 2015 it was estimated that within Belgium and the Netherlands only about $6,6 \%$ of all eligible candidates has been implanted with a CI (De Raeve, 2015).

In the Netherlands (population 17 million) 4943 adults and 1714 children received a CI by the end of 2017 (OPCI, 2018). Even though CI selection criteria generally ask for a bilateral severe hearing loss, current reimbursement regulations in the Netherlands, as in many other countries around the world, only support unilateral cochlear implantation. Given the high costs associated with cochlear implantation (about €40 000 (Smulders et al, 2016)), bilateral cochlear implants are only reserved for children and a small group of adults with special indications (e.g. acute cochlear obliteration, severe visual impairment). Only about $10 \%$ of the CI population in the Netherlands consists of bilateral implantees (OPCI, 2018).

In case of severe sensorineural hearing loss, acoustic amplification has it limits and electric stimulation has proven its benefits. Consequently, the border between profiting from a conventional hearing aid and the potential of retrieving additional benefit from a cochlear implant has shifted. CI candidacy criteria have broadened from profound towards severe hearing loss (Gifford et al, 2010; Hughes et al, 2014), increasing eligibility of asymmetric hearing losses (Firszt et al, 2018; van Loon et al, 2017). New candidacy criteria entail new research questions related to the continuously changing CI population. 
Indeed, broadening of CI criteria implies that many cochlear implant candidates today have usable residual hearing in the contralateral ear. Combined with the fact that $90 \%$ of CI candidates receive a CI in only one ear, the population of eligible candidates for bimodal hearing, making use of a $\mathrm{CI}$ in one ear and a conventional hearing aid in the other ear, has increased. The patient preference regarding bimodal hearing therefore was a current and clinically relevant question. As revealed in Chapter one, a recent CI-population showed a rather high bimodal hearing aid retention rate of $64 \%$ one year after implantation, while most studies in earlier years reported a bimodal use of only about 10 to 25\% (Fitzpatrick et al, 2009; Syms et al, 2002; Tyler et al, 2002; Yamaguchi \& Goffi-Gomez, 2013).

\section{THE PROFLLE OF A BIMODAL USER}

The question arises how we can identify those CI candidates that would profit from a bimodal fitting in order to optimize counseling of eligible CI-candidates in clinical practice. Given current reimbursement regulations one often has to make a choice on which ear to implant, minding a chance to profit from bimodal hearing. Often no strong medical (contra)indications exist to implant one ear over the other. Preoperative functional hearing as well as a more comparable performance outcome between both ears have shown to be indicative factors of patient preference for bimodal hearing (Chapter 1). Therefore these factors are important to weigh when counseling CI candidates towards the best possible outcome. Especially in the light of research initiatives questioning current reimbursement regulations and investigating the benefits and associated cost-effectiveness of bilateral cochlear implantation (Smulders et al, 2016; Yawn et al, 2018), it is important to get more information on the composition of the current bimodal population in the first place (Chapter 1). If bilateral CIs might become widely available, the challenge will be to decide which devices warrant the best possible outcome given the known benefits of the bilateral and bimodal combination.

Over the years it can be seen that the characteristics of the CI population, and as a consequence the bimodal population, has changed (Chapter 1). More subjects have aidable residual hearing over a wider frequency range. The investigated bimodal population in this thesis showed aidable residual hearing up until $1 \mathrm{kHz}$ (Chapter 1-4). It has however been shown that not purely sound sensitivity or detection thresholds, but moreover the capacities of the residual hearing in terms of functionality or intelligibility have a major role in becoming a bimodal user (Chapter 1). It was also seen that not the degree of hearing loss but rather the functional quality of the hearing aid experience prior to receiving a CI differed between unilateral and bimodal listeners (Chapter 2). Improved speech perception was the most reported reason for bimodal users to retain their contralateral 
hearing aid (Chapter 2) and bimodal benefits in speech perception could be objectified using a composed test battery (Chapter 3). Speech perception is known to depend on the processing of suprathreshold information demanding a good frequency and temporal resolution, loudness and fine structure representation, as well as top-down cognitive abilities. Given an intelligibility score between 14 and $50 \%$ in the contralateral ear, the benefit of the bimodal combination should be considered (Chapter 1). If the contralateral intelligibility score rises above $50 \%$, one stands a good chance of becoming a bimodal user after receiving a CI (Chapter 1). This criterion falls in line with the clinical procedure that often is applied when advising patients on the added value of a HA in the worse ear in case of a strong asymmetric hearing loss across ears or single-sided deafness.

Functional residual hearing could only explain part of the variation in patient preference regarding the bimodal combination (Chapter 1). It can be expected that other factors such as personal expectations, demands of the environment, aesthetics, costs or other patient characteristics could have an influence on bimodal hearing aid retention. It has for example been demonstrated that psychological factors play an essential role in the satisfaction of CI recipients (Kobosko et al, 2015). We found that the fitted outcome, the perceived handicap and the overall health status is comparable between a group of unilateral and bimodal listeners (Chapter 2). These findings could be named to suggest that the benefit of the bimodal combination in daily life listening is limited. It is however important to realize that the unilateral group and the bimodal group are most probably different in nature. The bimodal subjects needed the combined fitting of $\mathrm{CI}$ and HA across ears to reach the same disability rating as the unilateral group who only used a CI in one ear. Nevertheless, the outcome with CI in itself did not differ between both groups on average (Chapter 1\&2). It was however demonstrated that the difference in outcome between both ears played a role in bimodal hearing aid retention (Chapter 1). Moreover it was suggested that CI expectations and the demands asked from the functional hearing could be higher for subjects in the bimodal group (Chapter 2). If the CI outcome is less satisfactory in everyday listening, this leaves room for the benefits of a contralateral hearing aid. When questioning the bimodal group in itself on the merits of one of the devices versus the bimodal combination, benefits are consistently reported across all daily listening situations (Chapter 2). Expectations, daily life activities and listening demands are still an underexposed factor in research using hearing related questionnaires. In the past years patient preference, related outcomes (Hughes et al, 2018), as well as the perspective of measuring capabilities of subjects in reaching the goals in life they which to accomplish, is gaining more interest within the field of healthcare evaluations (van Hoof et al, 2015). 


\section{THE ORIGINS OF BIMODAL BENEFIT}

By definition, bimodal hearing is associated with asymmetric hearing. For most subjects the CI ear is the primary input for speech perception, whereby the contralateral HA may be seen as a favorable side effect since speech intelligibility often drops below 50\% (Chapter 1-4). Moreover, asymmetric differences regarding timing, loudness and frequency representation are inherently present across ears giving the origin of acoustic versus electrical hearing (Francart \& McDermott, 2013). These differences possibly restrain the benefit from binaural cues. Despite the asymmetric deficits, an asymmetric situation also gives rise to the unique opportunity of redundancy and complementarity. Low frequent acoustic hearing encloses cues regarding voice fundamental frequency, prosody, music and more, information that (currently) cannot be captured within the domain of electrical stimulation. Furthermore, the human brain is known to be perfectly capable of analyzing and combining redundant sound information (Litovsky, 2015). Overall, the opportunities prove to conquer the pitfalls, since it has been confirmed that bimodal aiding indeed enhances multiple dimensions of speech perception, i.e. intelligibility, listening effort and sound quality (Chapter 3) in all everyday listening situations (Chapter 2). In no case bimodal interference or decrement could be objectified (Chapter 3).

The degree of bimodal benefit however appeared to be quite capricious across bimodal listeners (Chapter 3). As in many other studies the amount of residual hearing alone was not found to be able to explain the differences in bimodal benefit (Chapter 3). Results indicate that not the bimodal integration of binaural cues, based on interaural differences, but rather the basic principles of bimodal summation and therefore the access to complementary information plays an important role in the quantity, the quality and the effort of speech perception (Chapter 3) as well as the benefit of bimodal directional microphones (Chapter 4). The exact origin of the substantial variability in bimodal benefits is however still unknown and future research is warranted to expose the principles behind the degree of bimodal benefit. The small sample sizes of most CI studies however often prohibit to investigate correlations between related factors with sufficient statistical power. Combining findings by meta-analysis into larger samples and prospective follow-up of subjects whereby patients act as their own control, should therefore be considered in future research.

\section{THE CLINICAL IMPLICATIONS}

The potential benefits of bimodal hearing are perhaps quite frequently overlooked since $50 \%$ of unilateral CI listeners reported to never have tried a contralateral HA 
(Chapter 2). Clinicians need to be mindful of the opportunities which exist for patients in terms of a tailored fitting. Even though further validation of such a practical implication in other and future populations is necessary, the presented guidelines on bimodal candidacy constitute a first step towards evidence-based bimodal hearing counseling in clinical practice (Chapter 1).

After counseling, the next step entails fitting the bimodal device combination. Research has shown that the fitting of the contralateral HA often is suboptimal (Harris \& Hay-McCutcheon, 2010; Yehudai et al, 2013) and that traditional fitting formulas in general support a good bimodal outcome (Vroegop et al, 2018b). Additionally, it has been demonstrated that when the CI and HA settings across ears are more adapted to each other, the benefit of the bimodal combination can be further increased. This involves the equalization of dynamic compression (Veugen et al, 2015), optimizing the frequency bandwidth according to audibility (Neuman et al, 2018), the enhancement of timing cues (Francart et al, 2014), loudness balancing (Veugen et al, 2016), as well as additional features such as bimodal directional microphones (Chapter 4). However, still more research by good quality comparative studies is needed to further develop evidence-based fitting procedures for bimodal hearing (Vroegop et al, 2018a).

It is apparent that having the $\mathrm{CI}$ and the HA fitted by different caregivers at different locations using different software programs, is unable to ensure the best bimodal alignment (Blamey \& Saunders, 2008). Despite the fact that the indication borders between $\mathrm{CI}$ and HA are fading and the bimodal combination is recognized as standard care, CI centers in many countries worldwide still do not actively engage in HA fittings (Scherf \& Arnold, 2014; Siburt \& Holmes, 2015). As in the Netherlands, CI clinicians focus on the fitting and rehabilitation of the CI, while the HA often is left at the same preoperative setting or subjects turn to their HA dispenser for adjustments of the HA settings. If anything has been advocated by research within the bimodal field, it is that the awareness of these issues and the reduction of these distances between $\mathrm{CI}$ and HA fitting should be addressed more actively in clinical practice.

In order to evaluate the bimodal fitting, it is essential to apply appropriate evaluation methods to quantify the achieved benefit. A test method should be able to map the true bimodal experience and measure the value of alterations or alternatives. Moreover it should be acceptably fast, clinically applicable and administrable to the total population of bimodal users. How a person listens, communicates, and interacts with his or her environment is far more complex than the commonly used basic speech intelligibility tasks in which patients repeat lists of words or sentences (McRackan et al, 2018). A bimodal test battery regarding 
speech perception is investigated (Chapter 3), showing that listening effort and sound quality are also important measures to consider. These measures did not only demonstrate to represent complementary dimensions from a theoretical perspective, but also proved to be quick and suitable for the total CI population (Chapter 3). Future efforts should be made to evaluate these measures in larger and other relevant populations in order to gain more insight into the underlying mechanisms and ways to improve them. Finally, patient related outcomes are gaining importance within the evaluation of health care interventions. A set of bimodal questionnaires was assembled, applied and evaluated in a clinical population (Chapter 2). (Reduced) versions of these questionnaires (e.g. SSQ-12) are recommended to be applied within clinical conditions in order to evaluate the subjective benefits of bimodal stimulation (Chapter 2).

\section{THE FUTURE OF BOTH WORLDS}

Subjects making use of the combination of electric-acoustic hearing bimodally as well as ipsilaterally (i.e. EAS stimulation) or every possible combination within and across ears, will become more relevant with broadening CI criteria. Moreover, it is thought that bilateral CIs will become more and more of a common practice. The trade-off between the benefit of the acoustic-electric complementarity versus the additional benefit gained by a second CI despite the high costs, is still an ongoing field of interest (Smulders et al, 2016; Yawn et al, 2018). Overall it is the aim to augment the performance of severely hearing impaired people. A unilateral CI is able to allow high levels of speech understanding in quiet conditions and can support conversations over the telephone. The performance of speech perception in complex noisy conditions is however still far from normal hearing capacities, even when supported with a contralateral HA (Chapter 3) and despite specially designed processing features (Chapter 4). Attempts should be made to further lower the effort of listening instead of the level of stagnation that is currently observed (Chapter 3).

In order to achieve a high level of speech perception, an adequate perception of pitch is required (Siciliano et al, 2010; Zhou et al, 2010). The frequency fitting of a CI nowadays however occurs in a universal manner, depicting the relevant frequency range of hearing in a standardized logarithmic manner across the electrode array, without reckoning with the individual natural tonotopy which in general is pitched about one octave lower (Chapter 5). Everyone practicing CI fittings will reckon that subjects profit from the regained access to the auditory world and most subjects over time adjust to the pitch misalignment. However, even after years of CI experience, listeners still judge the sound quality of their CI to be tinny or metallic (Chapter 2). Improving the place-pitch alignment in CI 
fitting therefore could perhaps not only improve speech perception, allow a faster learning phase, but also improve the quality of the sound experience. It is believed that especially in the situations where acoustic and electric stimulation is combined, as is the case with bimodal hearing, a better frequency alignment between both worlds could augment the complementary performance as well as the listening experience. The development of a new 3D analysis technique to identify the intracochlear location of $\mathrm{CI}$ electrodes more accurately allows us to predict a natural frequency map (Chapter 5). This map can be used in fitting the frequency allocation table of a CI. A large prospective clinical trial is warranted in order to compare the natural fitted frequency map with the standard frequency alignment. Hereby it is important that subjects act as their own control and are followed from the beginning of their rehabilitation in order to compare the learning curve between both maps while preventing first order preference. Special attention should be paid to those subjects who retain a contralateral HA as to map the perceived electro-acoustic sound image across ears and the effects it might entail regarding bimodal benefit.

Bimodal hearing brings acoustic and electric stimulation together across ears. Optimizing the combination between both worlds is however still an ongoing field of search. It could be speculated that with selection criteria shifting towards asymmetric hearing (Firszt et al, 2018; van Loon et al, 2017), more room for residual hearing in the non-implanted ear could possibly shift benefits from basic complementarity towards more true collaboration and fusion across ears. Results found so far advocate for further bimodal cooperation (Chapter 4). The future of bimodal hearing can only open new doors when a combined fitting procedure, a more enhanced inter-device communication and synchronized operation becomes available between CI and HA. Secondly, the implementation and translation of such bimodal fitting recommendations should find its way into clinical practice. This implies the simultaneous fitting of both devices by the same clinician, using the same software, allowing comparable settings in both devices in order to fully benefit from alignment and complementarity of timing, loudness and frequency information across ears. Moreover real-time exchange of information regarding the settings related to the current sound scene as well as streaming of the actual sound between both devices can open even more opportunities.

In order to improve hearing for the severely hearing impaired, efforts should be continued in order to maximize the benefit from both acoustic as well as electric stimulation and allow people to profit from the best combination of both worlds. 



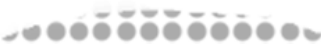

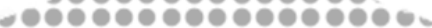

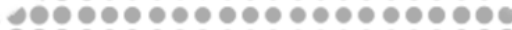

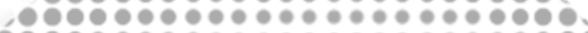

,

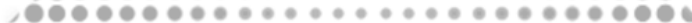

,

우

ر우

우

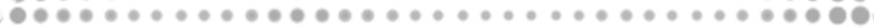

เ

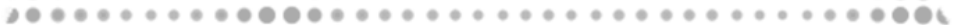

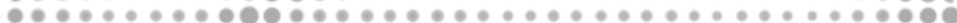

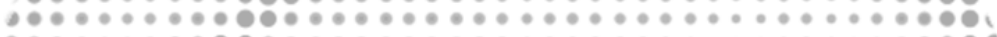

,

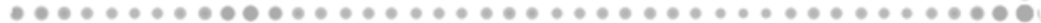

,

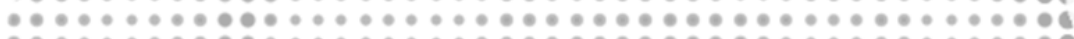



l

,

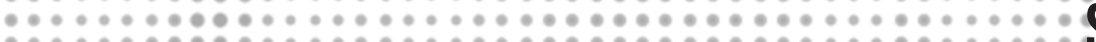

웅ำ

b

눈

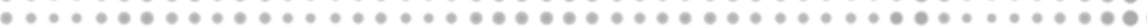

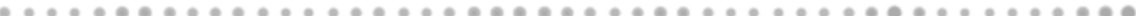

, . +

,

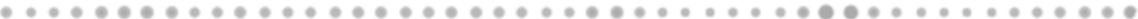

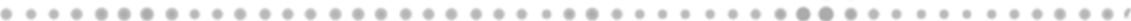

,

,

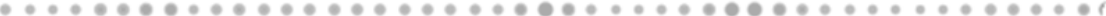

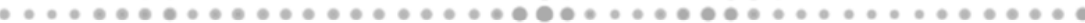

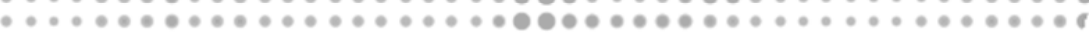

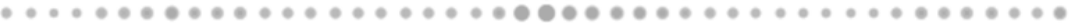

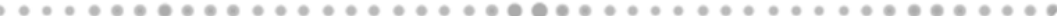

- . +

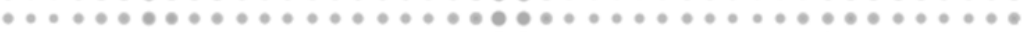

$\circ$

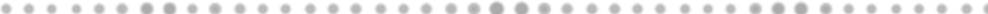

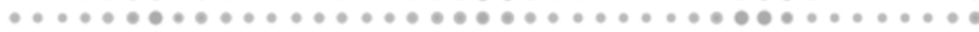

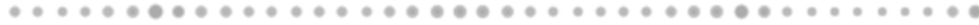

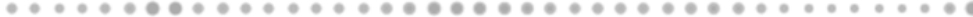

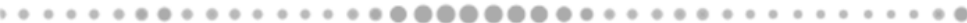

..............

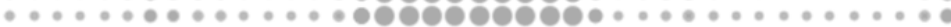

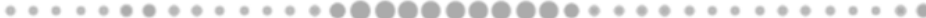

. . . * *......

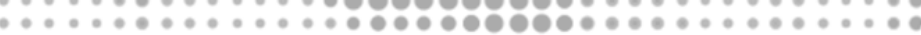

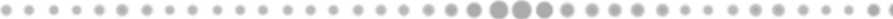

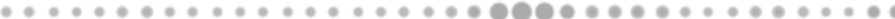

… *.............

..................

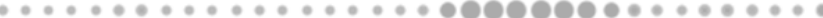

….............

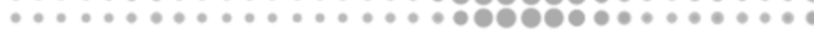

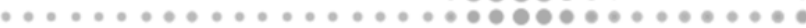

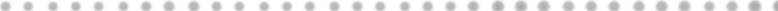

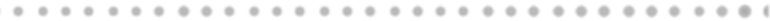

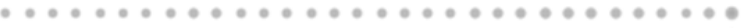

$\circ \cdots$

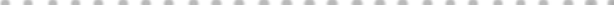

............................

,

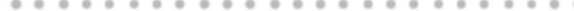

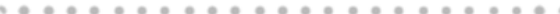

.......................

,

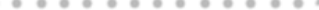

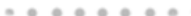





\section{BACKGROUND}

Cochlear implantation (CI) is considered standard of care to restore the access to sound in severely hearing impaired subjects. With inclusion criteria broadening over the years, the number of $\mathrm{CI}$ candidates keeps increasing. Many still have useful residual hearing in the non-implanted ear and can therefore be fitted with a conventional hearing aid (HA). When electric hearing by means of a $\mathrm{CI}$ in one ear is supplemented with acoustic hearing by use of a conventional HA in the opposite ear, one speaks of bimodal hearing.

Many researchers have demonstrated the benefit from a HA in bimodal listeners. Yet survey studies show that laboratory tests do not always relate to how CI recipients rate their abilities in everyday situations. Moreover little research has been carried out to assess which unilateral $\mathrm{CI}$ recipients are most likely to become bimodal users in the first place. Addressing the occurrence of bimodal use and the experiences of bimodal users in daily life are however very relevant topics in counseling unilateral $\mathrm{CI}$ recipients and providing them with a tailored fitting.

Concerning outcomes, it is known that speech perception in noise still remains one of the most challenging tasks for $\mathrm{CI}$ recipients. This refers not only to the intelligibility of the speech but also to the effort it takes to trace speech amongst competing noise and the quality of the sound that is perceived by the CI user. Nowadays directional microphone systems are accessible for HA as well as CI. Both bimodal hearing and directional microphone systems are proven ways to improve performance in noise. They are considered to be complementary, but however have not yet been evaluated conjointly.

Finally, the CI listening experience can possibly further be improved by optimizing its frequency representation, which differs from the natural acoustic pitch percept. It is known that $\mathrm{CI}$ recipients can, to a certain extent, adjust to this mismatch and can achieve good levels of speech intelligibility. Yet it is still unclear what could be the potential if the CI fitting better follows the natural tonotopy, especially when electric hearing is combined with natural residual hearing. In the case of bimodal hearing, frequency matched input across ears could augment the bimodal benefit based on bilateral and binaural cues.

\section{OBJECTIVES}

The general aim of the presented research was to gain more insight into the field of bimodal aiding. The following objectives were specifically dealt with in the consecutive chapters of this thesis: 
(1) Investigate the occurrence of a unilateral CI recipient becoming a bimodal user

(2) Assess bimodal experiences in daily life listening situations

(3) Measure bimodal benefit on speech perception across the range of bimodal users

(4) Evaluate monaural beamforming in bimodal recipients to improve speech perception in noise

(5) Explore the place-pitch mismatch between electric hearing and natural acoustic hearing

\section{METHODS}

A research project was carried out among the population of adult unilateral CI recipients at Maastricht University Medical Center. (1) A retrospective cohort chart review investigated the characteristics of those patients who continued or discontinued the use of their contralateral HA. (2) Secondly, a set of bimodal selfassessment questionnaires was sent out to query the daily life hearing experiences between both groups as well as within the bimodal group itself. (3) Thereafter a subset of subjects within the bimodal group was tested using a composed bimodal test battery measuring the degree of benefit from the HA aside the CI on different dimensions of speech perception, namely intelligibility, listening effort and sound quality. (4) Finally a subgroup of bimodal listeners was fitted with the same CI speech processor and a state-of-the art $\mathrm{HA}$ as to evaluate different directional microphone configurations when activated in the CI and/or the HA. (5) In order to explore the electric-acoustic place-pitch mismatch, the feasibility of a new calculation method using 3D imaging was investigated by comparing it to the pitch-matching task performed by a single sided deaf CI recipient.

\section{RESULTS}

The database study in Chapter (1) demonstrated a bimodal HA retention rate of more than $60 \%$ one year after receiving a unilateral CI. Continuing the use of a contralateral HA was significantly associated with better pure-tone thresholds and unaided speech scores in the non-implanted ear, as well as a smaller difference in speech recognition scores between both ears. A combined model of these factors related to bimodal HA retention was presented. Discrimination values were proposed as to identify which unilateral CI recipients are most likely to become bimodal users.

The questionnaire study in Chapter (2) showed that unilateral CI recipients who did not use a contralateral HA score their self-rated disability, hearing handicap and health related quality of life no different than those who did make use of 
bimodal aiding. However, when hearing (dis)ability was questioned within the group of bimodal listeners for the different listening situations (CI only, HA only, CIHA together), bimodal benefit could consistently be observed across various daily life hearing situations.

Chapter (3) presented the results of a bimodal test battery for speech perception. Speech intelligibility scores in quiet were better when performed bimodally compared to listening with $\mathrm{CI}$ or HA alone. Bilateral and binaural benefits of bimodal aiding were determined for speech intelligibility in spatially separated noise situations. At high signal-to-noise ratios, listening bimodally was shown to require less effort than listening with the $\mathrm{CI}$ alone. When rating the experienced sound quality of their bimodal hearing configuration, subjects judged it to sound significantly more voluminous and brighter than the $\mathrm{HA}$ alone and more voluminous, less unpleasant and less tinny compared to listening with the CI alone.

Chapter (4) illustrated that the application of a monaural beamformer in bimodal listeners could provide a substantial benefit for speech intelligibility in stationary as well as fluctuating noise. Most benefit was seen when beamforming was activated symmetrically in both CI and HA. Listening effort did not show an effect of directivity on top of speech intelligibility. Despite the benefits of directional microphones, there still remained a large difference between the performance of bimodal CI users and normal hearing listeners.

A proof-of-principle study in Chapter (5) revealed that the electrically evoked pitch percept matched well with the calculated frequency using the presented 3D imaging method. The new method reduced the mismatch compared to the conventional imaging method and was established as a valuable tool to predict the pitch percept on corresponding cochlear electrode positions. By means of the 3D imaging method the individual electric frequency allocation can be estimated in order to better resemble the natural acoustic tonotopy.

\section{CONCLUSIONS}

The majority of unilateral $\mathrm{CI}$ recipients continued to use a conventional HA after implantation. Using a HA aside the CI provided substantial benefits in diverse daily life hearing situations. Bimodal benefits were objectively demonstrated across extended dimensions of speech perception. Performance in noise could further be improved by implementing directional microphone systems in both CI and HA. It is hypothesized that reducing the place-pitch mismatch between electric and acoustic hearing can further improve the bimodal experience. Efforts should be continued to maximize the profit of combining the acoustic and electric worlds of hearing. 



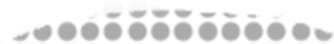

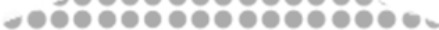

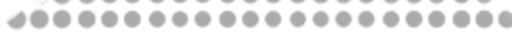

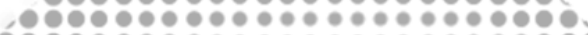

,

,

́,

6...........................

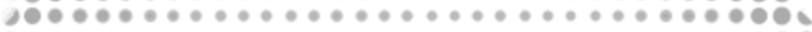

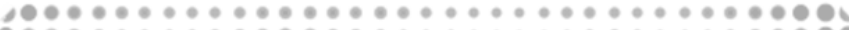

ㄴ․

เ

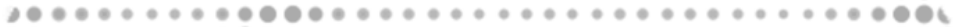

te.

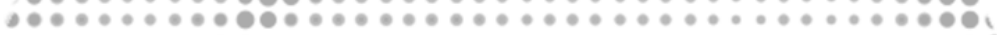

,

$\circ \circ$

,

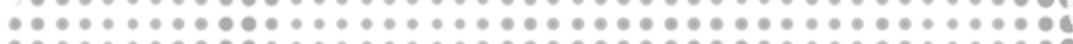

,

bis

,

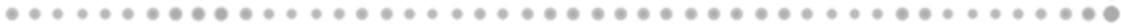

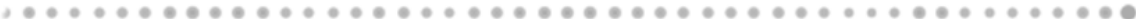

, ․ -

, * +

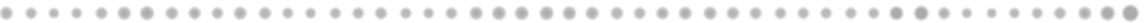

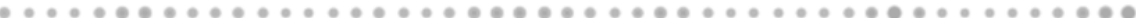

.

,

․ -

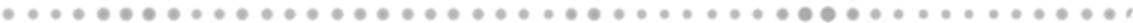

,

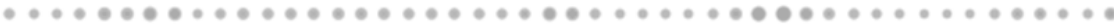

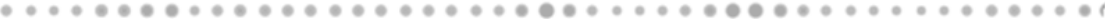

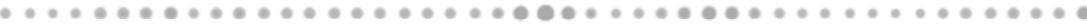

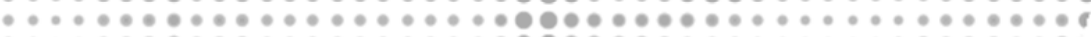

.

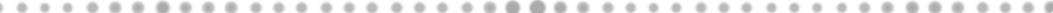

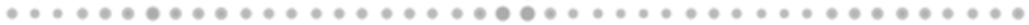

․

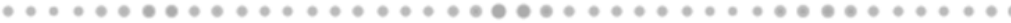

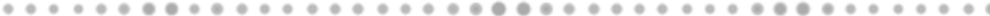

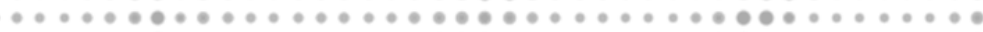

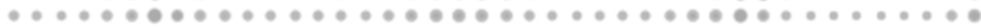

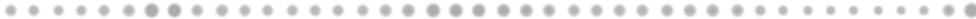

. . .

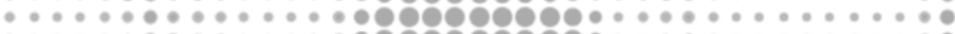

..... • *......

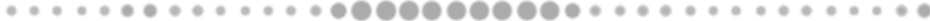

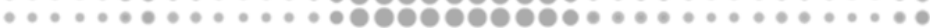

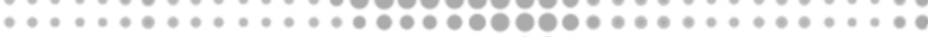

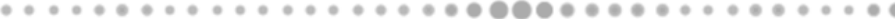

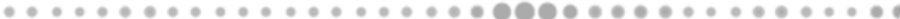

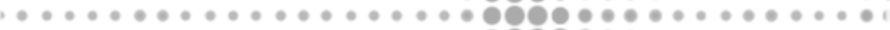

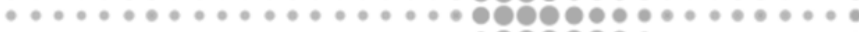

…*...........

.................

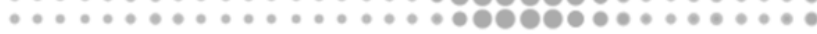

...... . ...........

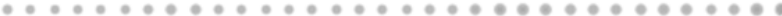

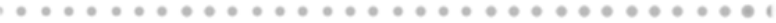

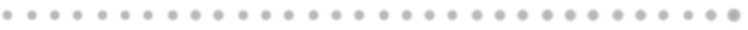

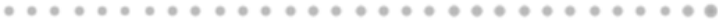

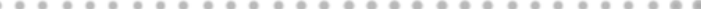

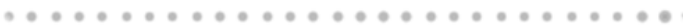

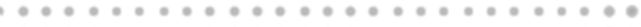

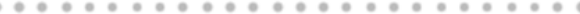

-

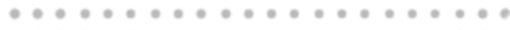

$+\cdots$

(10.

$\because, 0.0$. 



\section{ACHTERGROND}

Cochleaire implantatie (CI) wordt beschouwd als een gebruikelijke behandeling om ernstig slechthorende personen opnieuw toegang te geven tot geluid. Doordat de selectiecriteria voor CI door de jaren heen werden verruimd, is het aantal CI kandidaten toegenomen. Veel van hen hebben nog bruikbaar restgehoor in het niet geïmplanteerde oor, waardoor er in dit oor een conventioneel hoortoestel (HT) kan worden gebruikt. Wanneer elektrisch horen door middel van een CI in het ene oor wordt gecombineerd met akoestisch horen door een hoortoestel in het andere oor, spreekt men van bimodaal horen.

Meerdere onderzoeken hebben reeds bewezen dat het hoortoestel een meetbare verbetering oplevert voor bimodale luisteraars. Vragenlijststudies tonen echter aan dat er geen één-op-één relatie bestaat tussen hoe patiënten presteren in testsituaties en hoe ze hun functioneren beoordelen in dagelijkse luistersituaties. Bovendien is er nog weinig onderzoek gedaan naar welke unilaterale CI gebruikers in de eerste plaats de meeste kans hebben om een bimodale gebruiker te worden. Het voorkomen van bimodaal gebruik en de ervaringen van bimodale luisteraars in het dagelijks leven zijn echter zeer relevante onderwerpen wanneer we unilaterale CI gebruikers willen adviseren richting de meest geschikte hooroplossing.

Het is bekend dat de waarneming van spraak in rumoerige situaties nog steeds de grootste uitdaging vormt voor CI gebruikers. Hierbij wordt niet alleen het verstaan van spraak bedoeld, maar ook de inspanning die het kost om spraak te volgen te midden van storende ruis en de kwaliteit van het geluid dat wordt waargenomen door de CI gebruiker. Vandaag de dag is de technologie van directionele microfoons beschikbaar in zowel hoortoestellen als Cl's. Zowel bimodaal horen als directionele microfoonsystemen zijn bewezen manieren om prestaties in ruis te verbeteren. Ze worden beschouwd als complementair, maar werden tot op heden nog niet in combinatie geëvalueerd.

Tenslotte kan de luisterervaring van CI gebruikers mogelijk verbeterd worden door het optimaliseren van de wijze waarop frequentie informatie op een bepaalde locatie in het slakkenhuis wordt afgebeeld. Deze verschilt namelijk van de natuurlijke wijze waarop toonhoogte akoestisch wordt waargenomen. Het is bekend dat CI gebruikers tot op zekere hoogte kunnen wennen aan dit verschil, waardoor ze toch goede resultaten qua spraakverstaan bereiken. Het is echter nog steeds onduidelijk wat de mogelijkheden zouden kunnen zijn als de CI instellingen de natuurlijke tonotopie beter zouden volgen. Dit is met name van belang wanneer elektrische stimulatie gecombineerd wordt met natuurlijk restgehoor. Input die qua frequentie overeenstemt tussen beide oren zou het bimodaal voordeel nog 
kunnen verhogen op basis van het beschikbaar worden van bilaterale en binaurale aanwijzingen.

\section{DOELSTELLINGEN}

Het algemene doel van het hier gepresenteerde onderzoek was om meer inzicht te verwerven binnen het onderzoeksgebied van bimodaal horen. De volgende doelstellingen kwamen specifiek aan bod binnen de opeenvolgende hoofdstukken van deze thesis:

(1) Nagaan welke unilaterale CI patiënten uiteindelijk een bimodale gebruiker worden

(2) Bimodale ervaringen in dagelijkse luistersituaties in kaart brengen

(3) De mate van bimodaal voordeel vaststellen voor wat betreft de perceptie van spraak in brede zin

(4)Een monauraal directioneel microfoonsysteem evalueren bij bimodale gebruikers

(5) Het plaats-toonhoogte verschil tussen elektrisch horen en natuurlijk akoestisch horen verkennen

\section{METHODES}

Er werd een onderzoeksproject uitgevoerd naar de populatie volwassen unilaterale CI-gebruikers in het Maastricht Universitair Medisch Centrum. (1) Een retrospectieve cohortstudie onderzocht de eigenschappen van patiënten die wel of net niet een hoortoestel bleven dragen in het contralaterale oor. (2) Een set van bimodale vragenlijsten werd uitgestuurd om het verschil in kaart te brengen wat betreft dagelijkse luisterervaringen tussen beide groepen van patiënten, evenals binnen de groep van bimodale gebruikers. (3) Vervolgens werd een deel van de bimodale gebruikers getest door middel van een bimodale testbatterij die werd samengesteld om de mate van bimodaal voordeel te meten over verschillende dimensies van spraakwaarneming, namelijk het verstaan, de luisterinspanning en de geluidskwaliteit. (4) Tot slot werd een deel van de groep bimodale gebruikers voorzien van dezelfde CI spraakprocessor en een modern hoortoestel om verschillende directionele microfoonconfiguraties in het CI en/of het hoortoestel te testen. (5) $\mathrm{Om}$ het elektrisch-akoestische plaats-toonhoogte verschil te verkennen, werd de mogelijkheid van een nieuwe rekenmethode op basis van 3D beeldvorming onderzocht. Het resultaat werd vergeleken met een taak betreffende toonhoogtewaarneming die werd uitgevoerd door een eenzijdig dove CI gebruiker. 


\section{ResultaAt}

De databasestudie in Hoofdstuk (1) toonde aan dat meer dan 60\% van de unilaterale CI gebruikers één jaar na implantatie een hoortoestel blijft dragen in het contralaterale oor. Het blijven dragen van een hoortoestel in het andere oor bleek significant gerelateerd aan betere toondrempels en betere spraakscores (zonder behulp van een hoortoestel) in het niet geïmplanteerde oor, evenals een kleinere verschilscore qua spraakverstaan tussen beide oren. Op basis van deze factoren werd een gecombineerd model gepresenteerd betreffende de kans op bimodaal HT gebruik. Discriminatiewaarden werden voorgesteld om te bepalen welke unilaterale CI patiënten de meeste kans hebben om bimodaal gebruiker te worden.

De vragenlijststudie in Hoofdstuk (2) toonde aan dat unilaterale CI patiënten zonder hoortoestel in het andere oor hun hoormogelijkheden, auditieve handicap en kwaliteit van leven niet anders beoordeelden dan de patiënten die wel een hoortoestel bleven dragen. Wanneer echter de hoormogelijkheden binnen de bimodale groep werden bevraagd betreffende het luisteren met CI alleen, met HT alleen en met CI en HT samen, kon er consistent bimodaal voordeel worden aangetoond over alle verschillende dagelijkse hoorsituaties heen.

De resultaten van de bimodale testbatterij voor spraakwaarneming werden gepresenteerd in Hoofdstuk (3). De score voor het verstaan van spraak in stilte was beter wanneer er bimodaal dan wanneer er enkel met CI of enkel met het hoortoestel geluisterd werd. Bilaterale en binaurale voordelen van bimodaal horen voor het verstaan van spraak met spatiaal gescheiden ruisbronnen werden vastgelegd. Bij hoge signaal-ruis-verhoudingen kostte bimodaal luisteren significant minder luisterinspanning dan wanneer geluisterd werd met het CI alleen. De kwaliteit van de geluidservaring met CI en HT samen werd door bimodale gebruikers beoordeeld als voller en helderder dan het HT alleen en voller, minder onaangenaam en minder blikkerig dan wanneer er geluisterd werd met het CI alleen.

Hoofdstuk (4) illustreerde dat de toepassing van een monaurale directioneel microfoon systeem bij bimodale gebruikers een substantieel voordeel kan opleveren voor het verstaan van spraak in zowel stationaire als fluctuerende ruis. Het meeste voordeel werd vastgesteld wanneer het directionele systeem symmetrisch werd geactiveerd in zowel het CI als het HT. Luisterinspanning toonde geen bijkomend voordeel van directionaliteit bovenop het spraakverstaan. Ondanks de voordelen van directionele microfoons en bimodaal horen bleef er nog 
steeds een groot verschil bestaan tussen de prestatie van bimodale CI patiënten en normaal horende luisteraars.

De verkennende studie in Hoofdstuk (5) toonde aan dat elektrisch opgewekte toonhoogtewaarneming goed overeenkwam met de berekende frequentie door gebruik te maken van de 3D beeldvormingstechniek. De nieuwe methode reduceerde het verschil in vergelijking met de conventionele beeldvormingsmethode en werd geschikt bevonden als instrument om de waarneming van toonhoogte te voorspellen op overeenkomstige cochleaire elektrodeposities. Door gebruik te maken van de 3D beeldvormingstechniek kan de individuele elektrische frequentie-allocatie beter geschat worden om op deze wijze beter overeen te komen met de natuurlijke akoestische tonotopie.

\section{CONCLUSIE}

De meerderheid van de unilaterale CI patiënten blijft na implantatie een conventioneel hoortoestel gebruiken in het andere oor. Het gebruik van een hoortoestel naast het CI leverde substantiële voordelen op voor diverse dagelijkse luistersituaties. Dit bimodaal voordeel werd objectief aangetoond voor verschillende dimensies van spraakperceptie, namelijk verstaan, luisterinspanning en geluidskwaliteit. Er werd vastgesteld dat directionele microfoonsystemen de prestatie in ruis nog verder kunnen verbeteren wanneer deze worden toegepast in zowel het CI als het hoortoestel. De hypothese werd gevormd dat het reduceren van het plaats-toonhoogte verschil tussen elektrisch en akoestisch horen een verdere verbetering kan opleveren van de bimodale luisterervaring. Resultaten pleiten voor verder bimodale samenwerking. We moeten inspanningen blijven leveren om de best mogelijke winst te halen uit de combinatie van de akoestische en elektrische hoorwereld. 


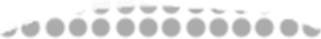

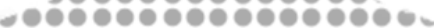

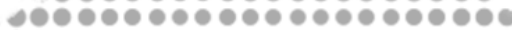

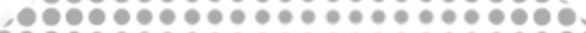

,

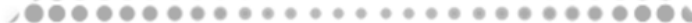

,

우

ر०

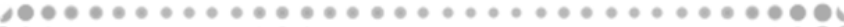

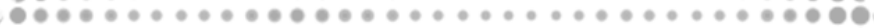

เ

분

te

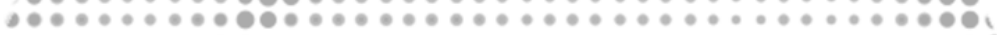

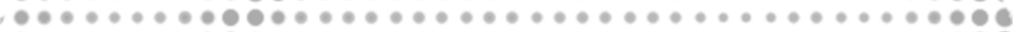

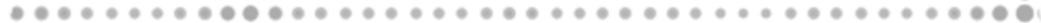

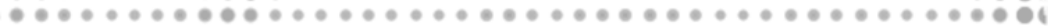

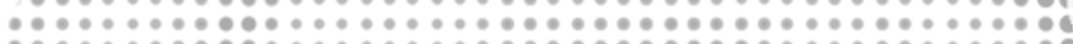

웅ำ

, e to

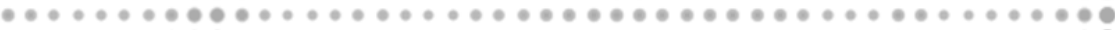

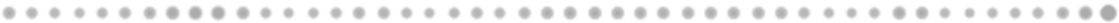

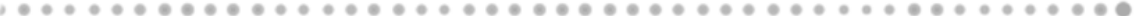

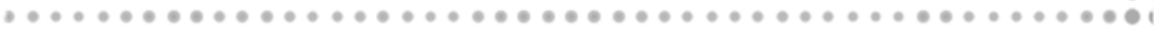

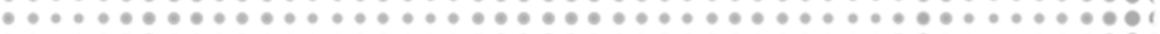

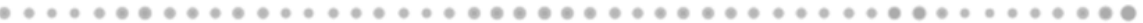

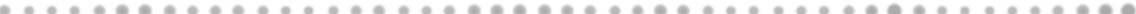

…

,

․

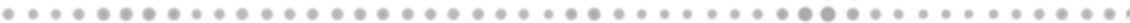

,

,

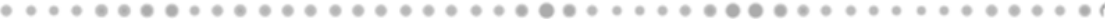

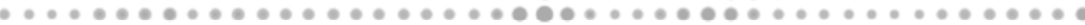

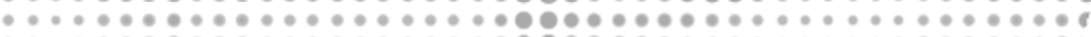

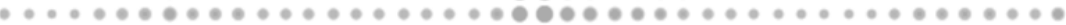

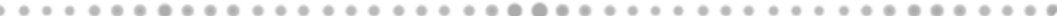

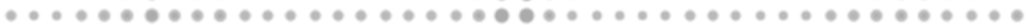

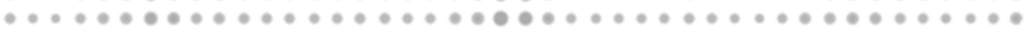

$\circ$

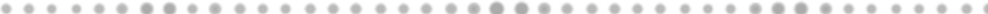

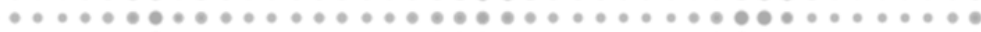

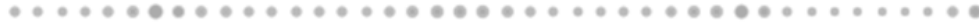

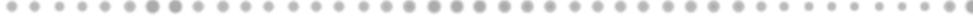

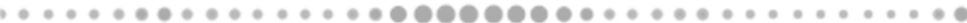

...... *.......

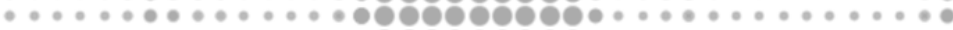

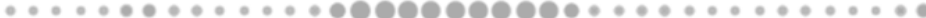

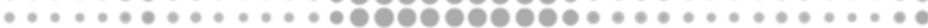

… +........

$\circ \ldots$

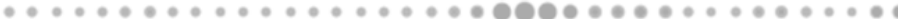

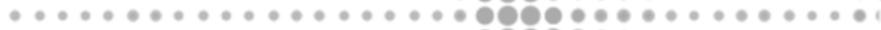

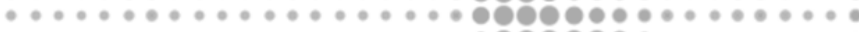

*..............

….............

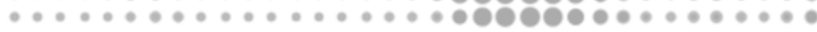

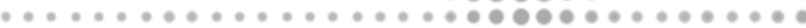

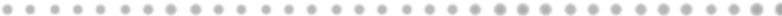

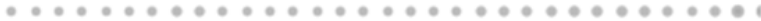

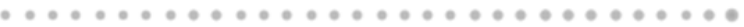

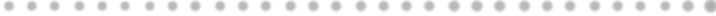

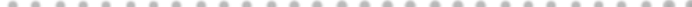

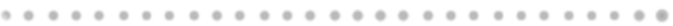

$\ldots \ldots \ldots$

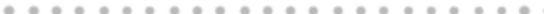

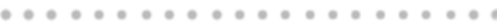

,

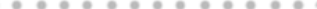

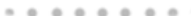



Advanced Bionics, 2012. White Paper: Clear Voice - Clinical Results. Available at: http://www.advancedbionics.com/con tent/dam/ab/Global/en_ce/documents /libraries/mediacenterdownloads/doc uments/ClearVoice White Paper.pdf.

Alexiades, G., Dhanasingh, A., Jolly, C., 2015. Method to Estimate the Complete and Two-Turn Cochlear Duct Length. Otol. Neurotol., 36(5), p.904-907.

Ali, H., Noble, J.H., Gifford, R.H., Labadie, R.F., Dawant, B.M., et al, 2015. Image-guided customization of frequency-place mapping in cochlear implants. In IEEE International Conference on Acoustics, Speech and Signal Processing (ICASSP). IEEE, pp. 5843-5847.

Amann, E., Anderson, I., 2014. Development and validation of a questionnaire for hearing implant users to self-assess their auditory abilities in everyday communication situations: the Hearing Implant Sound Quality Index (HISQUI19). Acta Otolaryngol., 134(9), p.915-23.

Amlani, A., 2001. Efficacy of directional microphone hearing aids: a metaanalytic perspective. J. Am. Acad. Audiol., 12(4), p.202-214.

ANSI S3.5, 1997. Methods For Calculation of The Speech Intelligilibity Index, New York: America National Standards Institute/Acoustical Society of America.

Appleton, J., König, G., 2014. Improvement in Speech Intelligibility and Subjective Benefit with Binaural Beamformer Technology. Hear. Rev., 21(11), p.4042.

Armstrong, M., Pegg, P., 1997. Speech perception in noise with implant and hearing aid. Am. J. Otol., 18(Suppl), p.S140-S141.

Arts, R., George, E., Griessner, A., Zierhofer, C., Stokroos, R., 2015. Tinnitus supression by intracochlear electrical stimulation in single sided deafness - a prospective clinical trial: Part I. Audiol. Neurotol. 20, p.294-313.

Avan, P., Giraudet, F., Büki, B., 2015.
Importance of binaural hearing. Audiol. Neurotol., 20(suppl 1), p.3-6.

Balfour, P., Hawkins, D., 1992. A comparison of sound quality judgments for monaural and binaural hearing aid processed stimuli. Ear Hear., 13(5), p.331-339.

Barbotte, E., Guillemin, F., Chau, N., Chau, N., Guillaume, S., et al, 2001. Prevalence of impairments, disabilities, handicaps and quality of life in the general population: A review of recent literature. Bull. World Health Organ., 79(11), p.1047-1055

Baumann, U., Nobbe, A., 2006. The cochlear implant electrode - pitch function. Hear. Res., 213(1-2), p.34-42.

Beijen, J.W., Mylanus, E.A.M., Leeuw, A.R., Snik, A.F.M., 2008. Should a hearing aid in the contralateral ear be recommended for children with a unilateral cochlear implant? Ann. Otol. Rhinol. Laryngol., 117(6), p.397-403.

Bentler, R., 2005. Effectiveness of directional microphones and noise reduction schemes in hearing aids: A systematic review of the evidence. J. Am. Acad. Audiol., 16(7), p.473-484.

Bentler, R., Egge, J., 2004. Quantification of directional benefit across different polar response patterns. J. Am. Acad. Audiol., 15(9), p.649-659.

Bernstein, J., Mehraei, G., 2013. Spectrotemporal modulation sensitivity as a predictor of speech intelligibility for hearing-impaired listeners. J. Am. Acad. Audiol., 24(4), p.293-306.

Bierer, J.A., Spindler, E., Bierer, S.M., Wright, R., 2016. An Examination of Sources of Variability Across the ConsonantNucleus-Consonant Test in Cochlear Implant Listeners. Trends Hear., 20.

Blamey, P., Artieres, F., Bașkent, D., Bergeron, F., Beynon, A., et al, 2012. Factors affecting auditory performance of postlinguistically deaf adults using cochlear implants: An update with 2251 patients. Audiol. Neurotol., 18(1), p.36-47. 
Blamey, P., Saunders, E., 2008. A review of bimodal binaural hearing systems and fitting. Acoust. Aust., 36(3), p.87-91.

Blamey, P.J., Maat, B., Baskent, D., Mawman, D., Burke, E., et al, 2015. A Retrospective Multicenter Study Comparing Speech Perception Outcomes for Bilateral Implantation and Bimodal Rehabilitation. Ear Hear., 36(4), p.408416.

Boëx, C., Baud, L., Cosendai, G., Sigrist, A., Kós, M.-I., et al, 2006. Acoustic to electric pitch comparisons in cochlear implant subjects with residual hearing. J. Assoc. Res. Otolaryngol., 7(2), p.110-124.

Boretzki, M., 1999. Quantification of significant sound quality attributes in the context of hearing instrument fine tuning. Phonak Hear. Syst. Focus \#25, p.3-11.

Bosman, A., Smoorenburg, G., 1995. Intelligibility of Dutch CVC syllables and sentences for listeners with normal hearing and with three types of hearing impairment. Audiology, 34(5), p.260284.

Boymans, M., 2003. Intelligent processing to optimize the benefits of hearing aids.

Boymans, M., Goverts, S.T., Kramer, S.E., Festen, J.M., Dreschler, W. a, 2008. A prospective multi-centre study of the benefits of bilateral hearing aids. Ear Hear., 29(6), p.930-941.

Brand, T., Kollmeier, B., 2002. Efficient adaptive procedures for threshold and concurrent slope estimates for psychophysics and speech intelligibility tests. J. Acoust. Soc. Am., 111(6), p.2801-2810.

Bredberg, G., Bredrerg, G., 1968. Cellular Pattern and Nerve Supply of the Human Organ of Corti. Acta Otolaryngol., Suppl 236, p.1-138.

Brockmeyer, A., 2011. Evaluation of Different Signal Processing Options in Unilateral and Bilateral Cochlear Freedom Implant Recipients Using R-Space ${ }^{\mathrm{TM}}$ Background Noise. J. Am. Acad. Audiol., 22(2), p.65-80.
Brons, I., Houben, R., Dreschler, W.A., 2012. Perceptual effects of noise reduction by time-frequency masking of noisy speech. J. Acoust. Soc. Am., 132(4), p.1690-1699.

Brons, I., Houben, R., Dreschler, W.A., 2013. Perceptual Effects of Noise Reduction With Respect to Personal Preference, Speech Intelligibility, and Listening Effort. Ear Hear., 34(1), p.29-41.

Brown, C.A., Bacon, S.P., 2010. Fundamental frequency and speech intelligibility in background noise. Hear. Res., 266(1-2), p.52-59.

Büchner, A., Schüssler, M., Battmer, R.D., Stöver, T., Lesinski-Schiedat, A., et al, 2009. Impact of low-frequency hearing. Audiol. Neurootol., 14(S1), p.8-13.

Buechner, A., Brendel, M., Saalfeld, H., Litvak, L., Frohne-Buechner, C., et al, 2010. Results of a pilot study with a signal enhancement algorithm for HiRes 120 cochlear implant users. Otol. Neurotol., 31(9), p.1386-1390.

Buechner, A., Dyballa, K.-H., Hehrmann, P., Fredelake, S., Lenarz, T., 2014 Advanced beamformers for cochlear implant users: acute measurement of speech perception in challenging listening conditions. PLoS One, 9(4), p.e95542.

Carlyon, R.P., Deeks, J.M., McKay, C.M., 2010a. The upper limit of temporal pitch for cochlear-implant listeners: Stimulus duration, conditioner pulses, and the number of electrodes stimulated. J. Acoust. Soc. Am., 127(3), p.1469-1478.

Carlyon, R.P., Lynch, C., Deeks, J.M., 2010b. Effect of stimulus level and place of stimulation on temporal pitch perception by cochlear implant users. $J$. Acoust. Soc. Am., 127(5), p.2997-3008.

Carlyon, R.P., Macherey, O., Frijns, J.H.M., Axon, P.R., Kalkman, R.K., et al, 2010c. Pitch Comparisons between Electrical Stimulation of a Cochlear Implant and Acoustic Stimuli Presented to a Normal-hearing Contralateral Ear. J. Assoc. Res. Otolaryngol., 11(4), p.625640. 
Chalupper, J., Agrawal, S., Fredelake, S., Spahr, T., 2013. Rationale and Implementation of a Bimodal Fitting Formula. , (Presented at CIAP Conference on Implantable Auditory Prostheses, Lake Tahoe, California, July 14-19, 2013). Available at http://figshare.com/articles/Rationale _and_Implementation_of_a_Bimodal_Fit ting_Formula/1340020.

Chang, Y., Chang, R.Y., Lin, C.-Y., Luo, X., 2016. Mandarin Tone and Vowel Recognition in Cochlear Implant Users. Ear Hear., 37(3), p.271-281.

Cheng, X., Liu, Y., Wang, B., Yuan, Y., Galvin, J.J., et al, 2018. The Benefits of Residual Hair Cell Function for Speech and Music Perception in Pediatric Bimodal Cochlear Implant Listeners. Neural Plast., p.1-10.

Ching, T., 2005. The evidence calls for making binaural-bimodal fittings routine. Hear. J., 58(11), p.32-41.

Ching, T., Incerti, P., Hill, M., van Wanrooy, E., 2006. An overview of binaural advantages for children and adults who use binaural/bimodal hearing devices. Audiol. Neurootol., 11(Suppl 1), p.6-11.

Ching, T., van Wanrooy, E., Dillon, H., 2007. Binaural-bimodal fitting or bilateral implantation for managing severe to profound deafness: a review. Trends Amplif, 11(3), p.161-192.

Ching, T.Y., van Wanrooy, E., Hill, M., Dillon, H., 2005. Binaural redundancy and interaural time difference cues for patients wearing a cochlear implant and a hearing aid in opposite ears. Int. J. Audiol., 44(9), p.513-521.

Ching, T.Y.C., Incerti, P., Hill, M., 2004. Binaural benefits for adults who use hearing aids and cochlear implants in opposite ears. Ear Hear, 25(1), p.9-21.

Christal, R.M., 2012. Subjective and Objective Measures of Adult Bimodal Users' Listening. Indep. Stud. Capstones, p.Paper 648. Available at: http://digitalcommons.wustl.edu/pacs _capstones/648.
Chung, K., Zeng, F., Acker, K., 2006. Effects of directional microphone and adaptive multichannel noise reduction algorithm on cochlear implant performance. J. Acoust. Soc. Americ, 120(4), p.22162227.

Cord, M., Surr, R., Walden, B., Dittberner, A., 2011. Ear asymmetries and asymmetric directional microphone hearing aid fittings. Am. J. Audiol., 20(2), p.111-122.

Cord, M., Walden, B., 2007. Field evaluation of an asymmetric directional microphone fitting. J. Am. Acad. Audiol., 18(3), p.245-256.

Cowan, R., Chin-Lenn, J., 2004. Pattern and prevalence of hearing aid use postimplantation in adult cochlear implant users. Aust NZJ Audiol, May(Suppl), p.48.

Cox, R., Hyde, M., Gatehouse, S., Noble, W., Dillon, H., et al, 2000. Optimal outcome measures, research priorities, and international cooperation. Ear Hear., 21(4 Suppl), p.106S-115S.

Cox, R.M., Alexander, G.C., 1995. The abbreviated profile of hearing aid benefit. Ear Hear., 16(2), p.176-86.

Crew, J.D., Galvin III, J.J., Landsberger, D.M., Fu, Q.-J., 2015. Contributions of Electric and Acoustic Hearing to Bimodal Speech and Music Perception. PLOS One, 10(3), p.e0120279.

Crew, J.D., Galvin, J.J., Fu, Q.J., 2016. Perception of Sung Speech in Bimodal Cochlear Implant Users. Trends Hear., 20, p.1-15.

Cullington, H.E., Zeng, F., 2011. Comparison of bimodal and bilateral cochlear implant users on speech recognition with competing talker, music perception, affective prosody discrimination, and talker identification. Ear Hear., 32(1), p.16-30.

D’Alessandro, H.D., Ballantyne, D., Boyle, P.J., De Seta, E., DeVincentiis, M., et al, 2018. Temporal fine structure processing, pitch, and speech perception in adult cochlear implant recipients. Ear Hear., 39(4), p.679-686. 
Dalton, D.S.D., Cruickshanks, K.J., Klein, B.E.K., Klein, R., Wiley, T.L., et al, 2003. The Impact of Hearing Loss on Quality of Life in Older Adults. Gerontologist, 43(5), p.661-668.

Davis, A., 1995. Hearing in Adults: The Prevalence and Distribution of Hearing Impairment and Reported Hearing Disability in the MRC Institute of Hearing Research's National Study of Hearing M. I. of H. Research, ed., Whurr.

Demeester, K., Topsakal, V., Hendrickx, J., Fransen, E., Laer, L. Van, et al, 2012. Hearing Disability Measured by the Speech, Spatial, and Qualities of Hearing Scale in Clinically Persons, and Disability Screening by Means of a Reduced SSQ ( the SSQ5 ). Ear Hear., 33(5), p.615-626.

Desjardins, J.L., 2016. The Effects of Hearing Aid Directional Microphone and Noise Reduction Processing on Listening Effort in Older Adults with Hearing Loss. J. Am. Acad. Audiol., 27(1), p.2941.

Devocht, E.M.J., George, E.L.J., Janssen, A.M.L., Stokroos, R.J., 2015. Bimodal Hearing Aid Retention after Unilateral Cochlear Implantation. Audiol. Neurotol., 20(6), p.383-393.

Devocht, E.M.J., Janssen, A.M.L., Chalupper, J., Stokroos, R.J., George, E.L.J., 2017. The Benefits of Bimodal Aiding on Extended Dimensions of Speech Perception: Intelligibility, Listening Effort, and Sound Quality. Trends Hear., 21.

Dillon, H., 2012a. Binaural and bilateral considerations in hearing aid fitting. In H. Dillon, ed. Hearing Aids. New York, NY: Thieme medical publishers.

Dillon, H., 2012b. Hearing Aids, Second Edition, Thieme Medical Publishers.

Dincer D’Alessandro, H., Sennaroğlu, G., Yücel, E., Belgin, E., Mancini, P., 2015. Binaural squelch and head shadow effects in children with unilateral cochlear implants and contralateral hearing aids. Acta Otorhinolaryngol. Ital., 35(5), p.343-9.
Dorman, M.F., Cook, S., Spahr, A., Zhang, T., Loiselle, L., et al, 2015. Factors constraining the benefit to speech understanding of combining information from low-frequency hearing and a cochlear implant. Hear. Res., 322, p.107-111.

Dorman, M.F., Gifford, R.H., 2017. Speech Understanding in Complex Listening Environments by Listeners Fit With Cochlear Implants. J. Speech, Lang. Hear. Res., 60(10), p.3019-3026.

Dorman, M.F., Gifford, R.H., Spahr, A.J., McKarns, S. a, 2008. The benefits of combining acoustic and electric stimulation for the recognition of speech, voice and melodies. Audiol. Neurootol., 13(2), p.105-112.

Dorman, M.F., Spahr, T., Gifford, R., Loiselle, L., McKarns, S., et al, 2007. An Electric Frequency-to-place Map for a Cochlear Implant Patient with Hearing in the Nonimplanted Ear. J. Assoc. Res. Otolaryngol., 8(2), p.234-240.

Durisin, M., Bartling, S., Arnoldner, C., Ende, M., Prokein, J., et al, 2010. Cochlear osteoneogenesis after meningitis in cochlear implant patients: a retrospective analysis. Otol. Neurotol., 31(7), p.1072-1078.

Ebrahimi-Madiseh, A., Eikelboom, R.H., Jayakody, D.M., Atlas, M.D., 2016. Speech perception scores in cochlear implant recipients: An analysis of ceiling effects in the CUNY sentence test (Quiet) in post-lingually deafened cochlear implant recipients. Cochlear Implants Int., 17(2), p.75-80.

Eisenberg, L.S., Dirks, D.D., Gornbein, J.A., 1997. Subjective judgments of speech clarity measured by paired comparisons and category rating. Ear Hear., 18(4), p.294-306.

Elko, G., Pong, A., 1995. Simple adaptive first order differential microphone. In IEEE ASSP Workshop on Applications of Signal Processing to Audio and Acoustics. New Paltz, NY, USA, pp. 169172. 
Ellis, R.J., Ronnberg, J., 2014. Cognition and speech-in-noise recognition: the role of proactive interference. J. Am. Acad. Audiol., 25(10), p.975-982.

van Esch, T.E.M., Kollmeier, B., Vormann, M., Lyzenga, J., Houtgast, T., et al, 2013. Evaluation of the preliminary auditory profile test battery in an international multi-centre study. Int. J. Audiol., 52(5), p.305-21.

Farinetti, A., Roman, S., Mancini, J., Baumstarck-Barrau, K., Meller, R., et al, 2015. Quality of life in bimodal hearing users (unilateral cochlear implants and contralateral hearing aids). Eur. Arch. Oto-Rhino-Laryngology, 272(11), p.3209-3215.

Farnik, M., Pierzchała, W.A., 2012. Instrument development and evaluation for patient-related outcomes assessments. Patient Relat. Outcome Meas., 3, p.1-7.

El Fata, F., James, C.J., Laborde, M.-L., Fraysse, B., 2009. How much residual hearing is "useful" for music perception with cochlear implants? Audiol. Neurootol., 14 Suppl 1(suppl 1), p.14-21.

Faul, F., Erdfelder, E., Lang, A., Buchner, A., 2007. G*Power 3: A flexible statistical power analysis program for social, behavioral, and biomedical sciences. Behav. Res. Methods, 39(2), p.175-191.

Faulkner, K.F., Pisoni, D.B., 2013. Some observations about cochlear implants: challenges and future directions. Neurosci. Discov., 1(1), p.9.

Fearn, R., Cartel, P., Wolfe, J., 1999. The perception of pitch by users of cochlear implants: Possible significance for rate and place theories of pitch. Acoust. Aust., 27(2), p.41-43.

Feeny, D., Furlong, W., Torrance, G.W., Goldsmith, C.H., Zhu, Z., et al, 2002. Multiattribute and single-attribute utility functions for the health utilities index mark 3 system. Med. Care, 40(2), p.113-128.

Fetterman, B.L., Domico, E.H., 2002. Speech recognition in background noise of cochlear implant patients. Otolaryngol.
Head. Neck Surg., 126(3), p.257-263.

Feuerstein, J.F., 1992. Monaural versus Binaural Hearing: Ease of Listening, Word Recognition, and Attentional Effort. Ear Hear., 13(2), p.80-86.

Fielden, C.A., Hampton, R., Smith, S., Kitterick, P.T., 2016. Access to aidable residual hearing in adult candidates for cochlear implantation in the UK. Cochlear Implants Int., 17 (sup1), p.70-73.

Firszt, J.B., 2008. Restoring hearing symmetry with two cochlear implants or one cochlear implant and a contralateral hearing aid. J. Rehabil. Res. Dev., 45(5), p.749-768.

Firszt, J.B., Reeder, R.M., Holden, L.K., Dwyer, N.Y., Gotter, B., et al, 2018. Results in Adult Cochlear Implant Recipients With Varied Asymmetric Hearing: A Prospective Longitudinal Study of Speech Recognition, Localization, and Participant Report. Ear Hear., p.1-18.

Fitzgerald, M.B., Sagi, E., Morbiwala, T. a, Tan, C.-T., Svirsky, M. a, 2013. Feasibility of real-time selection of frequency tables in an acoustic simulation of a cochlear implant. Ear Hear., 34(6), p.763-772.

Fitzpatrick, E.M., Leblanc, S., 2010. Exploring the factors influencing discontinued hearing aid use in patients with unilateral cochlear implants. Trends Amplif., 14(4), p.199-210.

Fitzpatrick, E.M., Seguin, C., Schramm, D., Chenier, J., Armstrong, S., 2009. Users' experience of a cochlear implant combined with a hearing aid. Int $J$ Audiol, 48(4), p.172-182.

Flynn, M.C., Schmidtke, T., 2004. Benefits of bimodal stimulation for adults with a cochlear implant. Int. Congr. Ser., 1273(November), p.227-230.

Francart, T., Lenssen, A., Wouters, J., 2014. Modulation Enhancement in the Electrical Signal Improves Perception of Interaural Time Differences with Bimodal Stimulation. J. Assoc. Res. Otolaryngol., 15(4), p.633-647. 
Francart, T., McDermott, H.J., 2013. Psychophysics, fitting, and signal processing for combined hearing aid and cochlear implant stimulation. Ear Hear., 34(6), p.685-700.

Francart, T., van Wieringen, A., Wouters, J., 2011. Comparison of fluctuating maskers for speech recognition tests. Int. J. Audiol., 50(1), p.2-13.

Frijns, J.H., Briaire, J.J., Grote, J.J., 2001. The importance of human cochlear anatomy for the results of modiolushugging multichannel cochlear implants. Otol. Neurotol., 22(3), p.340349.

Frohne-Büchner, C., Büchner, A., Gärtner, L., Battmer, R.D., Lenarz, T., 2004. Experience of uni- and bilateral cochlear implant users with a microphone positioned in the pinna. Int. Congr. Ser., 1273, p.93-96.

Fu, Q.J., Nogaki, G., 2005. Noise susceptibility of cochlear implant users: The role of spectral resolution and smearing. JARO - J. Assoc. Res. Otolaryngol., 6(1), p.1927.

Gabrielsson, A., 1979. Dimension analyses of perceived sound quality of soundreproducing systems. Scand. J. Psychol., 20, p.159-169.

Gatehouse, S., Akeroyd, M., 2006. Two-eared listening in dynamic situations. Int. J. Audiol., 45 Suppl 1(Supplement 1), p.S120-4.

Gatehouse, S., Noble, W., 2004. The Speech , Spatial and Qualities of Hearing Scale ( SSQ ). Int. J. Audiol., 43(2), p.85-99.

Geissler, G., Arweiler, I., 2014. Speech reception threshold benefits in cochlear implant users with an adaptive beamformer in real life situations. Cochlear Implants Int., 16(2), p.69-76.

George, E., Festen, J., Houtgast, T., 2006. Factors affecting masking release for speech in modulated noise for normalhearing and hearing-impaired listeners. J. Acoust. Soc. Am., 120(4), p.22952311.
George, E.L.J., Zekveld, A.A., Kramer, S.E., Goverts, S.T., Festen, J.M., et al, 2007. Auditory and nonauditory factors affecting speech reception in noise by older listeners. J. Acoust. Soc. Am., 121(4), p.2362-75.

Gifford, R., Revit, L., 2010. Speech perception for adult cochlear implant recipients in a realistic background noise: effectiveness of preprocessing strategies and external options for improving speech recognition in noise. J. Am. Acad. Audiol., 21(7), p.441-488.

Gifford, R.H., Dorman, M.F., 2012. The psychophysics of low-freqeuncy acoustic hearin gin electric and acoustic stimulation (EAS) and bimodal patients. J. Hear. Sci., 2(2), p.33-44.

Gifford, R.H., Dorman, M.F., McKarns, S.A., Spahr, A.J., 2007. Combined electric and contralateral acoustic hearing: Word and sentence recognition with bimodal hearing. J Speech Lang Hear Res, 50(4), p.835-843.

Gifford, R.H., Dorman, M.F., Shallop, J.K. Sydlowski, S.A., 2010. Evidence for the expansion of adult cochlear implant candidacy. Ear Hear., 31(2), p.186-194.

Gifford, R.H., Dorman, M.F., Sheffield, S.W., Teece, K., Olund, A.P., 2014. Availability of Binaural Cues for Bilateral Implant Recipients and Bimodal Listeners with and without Preserved Hearing in the Implanted Ear. Audiol. Neurotol., 19(1), p.57-71.

Gifford, R.H., Shallop, J.K., Peterson, A.M., 2008. Speech recognition materials and ceiling effects: Considerations for cochlear implant programs. Audiol. Neurotol., 13(3), p.193-205.

Glasberg, B., Moore, B., 1989. Psychoacoustic abilities of subjects with unilateral and bilateral cochlear hearing impairments and their relationship to the ability to understand speech. Scand. Audiol., 32, p.1-25.

Goman, A.M., 2014. A comparison of bilateral cochlear implantation and bimodal aiding in severely-profoundly hearingimpaired adults: head movements, 
clinical outcomes, and costeffectiveness. University of York.

Goupell, M.J., Stoelb, C., Kan, A., Litovsky, R.Y., 2013. Effect of mismatched place-ofstimulation on the salience of binaural cues in conditions that simulate bilateral cochlear-implant listening. $J$. Acoust. Soc. Am., 133(4), p.2272-2287.

Graham, J.W., 2009. Missing Data Analysis: Making It Work in the Real World. Annu. Rev. Psychol., 60(1), p.549-576.

Graham, J.W., Olchowski, A.E., Gilreath, T.D., 2007. How many imputations are really needed? Some practical clarifications of multiple imputation theory. Prev. Sci., 8(3), p.206-213.

Grancharov, V., Kleijn, W., Tobergte, D.R., Curtis, S., 2007. Sound Quality Assessment. In J. Benesty, M. Sondhi, \& Y. Huang, eds. Springer HandBook of Speech Processing. Berlin: Springer, pp. 83-99.

Grant, K., Walden, T., 2013. Understanding excessive SNR loss in hearing-impaired listeners. J. Am. Acad. Audiol., 24(4), p.329-336.

Green, T., Faulkner, A., Rosen, S., 2012 Frequency selectivity of contralateral residual acoustic hearing in bimodal cochlear implant users, and limitations on the ability to match the pitch of electric and acoustic stimuli. Int $J$ Audiol, 51(5), p.389-398.

Greenwood, D.D., 1990. A cochlear frequencyposition function for several species-29 years later. J. Acoust. Soc. Am., 87(6), p.2592-2605.

Grutters, J.P.C., Joore, M. a, van der Horst, F., Verschuure, H., Dreschler, W. a, et al, 2007. Choosing between measures: comparison of EQ-5D, HUI2 and HUI3 in persons with hearing complaints. Qual. Life Res., 16(8), p.1439-49.

Guérit, F., Santurette, S., Chalupper, J., Dau, T., 2014. Investigating Interaural Frequency-Place Mismatches via Bimodal Vowel Integration. Trends Hear., 18, p.1-10.
Hallberg, L.R.-M., Hallberg, U., Kramer, S.E., 2008. Self-reported hearing difficulties, communication strategies and psychological general well-being (quality of life) in patients with acquired hearing impairment. Disabil. Rehabil., 30(3), p.203-212.

Hällgren, M., Larsby, B., Lyxell, B., Arlinger, S., 2005. Speech understanding in quiet and noise, with and without hearing aids. Int. J. Audiol., 44(June), p.574-583.

Hamzavi, J., Pok, S.M., Gstoettner, W., Baumgartner, W.-D., 2004. Speech perception with a cochlear implant used in conjunction with a hearing aid in the opposite ear. Int. J. Audiol., 43(2), p.61-65.

Hanley, J., McNeil, B., 1982. The meaning and use of the area under a receiver operating characteristic (ROC) curve. Radiology, 143(1), p.29-36.

Harlander, N., Rosenkranz, T., Hohmann, V., 2012. Evaluation of model-based versus non-parametric monaural noise-reduction approaches for hearing aids. Int. J. Audiol., 51(8), p.627-39.

Harris, M.S., Hay-McCutcheon, M., 2010. An analysis of hearing aid fittings in adults using cochlear implants and contralateral hearing aids. Laryngoscope, 120(12), p.2484-2488.

Hay-McCutcheon, M.J., Peterson, N.R., Pisoni, D.B., Kirk, K.I., Yang, X., et al, 2018. Performance variability on perceptual discrimination tasks in profoundly deaf adults with cochlear implants. J. Commun. Disord., 72(2), p.122-135.

Hersbach, A. a, Arora, K., Mauger, S.J., Dawson, P.W., 2012. Combining directional microphone and single-channel noise reduction algorithms: a clinical evaluation in difficult listening conditions with cochlear implant users. Ear Hear., 33(4), p.e13-23.

Van de Heyning, P.H., Vermeire, K., Cochet, E., Hofkens, A., De Bodt, M., 2004. Cochlear implantation: a bionic system to restore hearing in severely hearing impaired patients. Verh. K. Acad. Geneeskd. Belg., 66(4), p.253-67. 
van Hoesel, R.J.M., 2012. Contrasting benefits from contralateral implants and hearing aids in cochlear implant users. Hear. Res., 288(1-2), p.100-13.

Holube, I., 2011. Speech intelligibility in fluctuating maskers. Available at: http://www.audiologicallibrary.gnresound.dk/External/GN/ral. nsf/0/575564BCD3422D8EC1257A0D 0042274E/\$FILE/57-64, Hubole, Speech intelligibility in fluctuating maskers, 2011.pdf.

van Hoof, M., Jeuring, S.F.G., Stokroos, R.J., Joore, M.A., 2015. [A new perspective on measuring quality of life using the capability approach]. Ned. Tijdschr. Geneeskd., 159(November), p.A9234.

Hoppe, U., Hocke, T., Digeser, F., 2018. Bimodal benefit for cochlear implant listeners with different grades of hearing loss in the opposite ear. Acta Otolaryngol., $0(0)$, p.1-9.

Hornsby, B.W.Y., Ricketts, T. a, 2007. Effects of noise source configuration on directional benefit using symmetric and asymmetric directional hearing aid fittings. Ear Hear., 28(2), p.177-86.

Houben, R., Dreschler, W.A., 2015. Optimization of the Dutch Matrix Test by Random Selection of Sentences From a Preselected Subset. Trends Hear., 19, p.1-10.

Houben, R., Koopman, J., Luts, H., Wagener, K.C., van Wieringen, A., et al, 2014. Development of a Dutch matrix sentence test to assess speech intelligibility in noise. Int. J. Audiol., 53(10), p.760-3.

House, W., 1976. Chapter I Cochlear Implants: Beginnings (1957-1961). Ann. Otol. Rhinol. Laryngol.

Hughes, M.L.M., Neff, D.D.L., Simmons, J.L., Moeller, M.P., 2014. Performance Outcomes for Borderline Cochlear Implant Recipients With Substantial Preoperative Residual Hearing. Otol. Neurotol., 35(8), p.1373-1384.

Hughes, S.E., Hutchings, H.A., Rapport, F.L., McMahon, C.M., Boisvert, I., 2018.
Social Connectedness and Perceived Listening Effort in Adult Cochlear Implant Users: A Grounded Theory to Establish Content Validity for a New Patient-Reported Outcome Measure. Ear Hear.

Huinck, W.J., Mylanus, E.A.M., Snik, A.F.M., 2019. Expanding unilateral cochlear implantation criteria for adults with bilateral acquired severe sensorineural hearing loss. Eur. Arch. Oto-RhinoLaryngology, $0(0)$, p.0.

Humes, L.E., 1999. Dimensions of hearing aid outcome. J. Am. Acad. Audiol., 10(1), p.26-39.

Humes, L.E., 2002. Factors underlying the speech-recognition performance of elderly hearing-aid wearers. J. Acoust. Soc. Am., 112(3), p.1112-1132.

Illg, A., Bojanowicz, M., Lesinski-Schiedat, A., Lenarz, T., Büchner, A., 2014. Evaluation of the bimodal benefit in a large cohort of cochlear implant subjects using a contralateral hearing aid. Otol. Neurotol., 35(9), p.e240-e244.

Iwaki, T., Matsushiro, N., Mah, S.-R., Sato, T., Yasuoka, E., et al, 2004. Comparison of speech perception between monaural and binaural hearing in cochlear implant patients. Acta Otolaryngol., 124(4), p.358-62.

Kaandorp, M.W., Smits, C., Merkus, P., Goverts, S.T., Festen, J.M., 2015. Assessing speech recognition abilities with digits in noise in cochlear implant and hearing aid users. Int. J. Audiol., 54(1), p.48-57.

Kalkman, R.K., Briaire, J.J., Dekker, D.M.T., Frijns, J.H.M., 2014. Place pitch versus electrode location in a realistic computational model of the implanted human cochlea. Hear. Res., 315, p.1024.

Kam, A.C.S., Ng, I.H.Y., Cheng, M.M.Y., Wong, T.K.C., Tong, M.C.F., 2012. Evaluation of the ClearVoice strategy in adults using hiresolution fidelity 120 sound processing. In Clinical and Experimental Otorhinolaryngology. pp. S89-S92. 
Keidser, G., Dillon, H., Convery, E., Mejia, J., 2013. Factors Influencing Individual Variation in Perceptual Directional Microphone Benefit. J. Am. Acad. Audiol., 24(10), p.955-968.

Keidser, G., Hartley, D., Carter, L., 2008. Longterm usage of modern signal processing by listeners with severe or profound hearing loss: a retrospective survey. Am. J. Audiol., 17(2), p.136-46.

Keidser, G., Seymour, J., 1999. An efficient, adaptive method of measuring loudness growth functions. Scand. Audiol., 28(1), p.3-14.

Kim, H., Kang, W.S., Park, H.J., Lee, J.Y., Park, J.W., et al, 2018. Cochlear Implantation in Postlingually Deaf Adults is Timesensitive Towards Positive Outcome: Prediction using Advanced Machine Learning Techniques. Sci. Rep., 8(1), p.18004.

Kim, J.S., Bryan, M.F., 2011. The effects of asymmetric directional microphone fittings on acceptance of background noise. Int. J. Audiol., 50(5), p.290-6.

Klasen, T.J., Moonen, M., 2005. Preservation of interaural time delay for binaural hearing aids through multi-channel Wiener filtering based noise reduction. Electr. Eng., (3), p.29-32.

Klink, K.B., Schulte, M., Meis, M., 2012a Measuring listening effort in the field of audiology - a literature review of methods, part 1. Zeitschrift für Audiol., 51(2), p.60-67.

Klink, K.B., Schulte, M., Meis, M., 2012b. Measuring listening effort in the field of audiology - a literature review of methods (part 2). Zeitschrift für Audiol., 51(2), p.60-67.

Kobosko, J., Jedrzejczak, W.W., Pilka, E., Pankowska, A., Skarzynski, H., 2015. Satisfaction with Cochlear Implants in Postlingually Deaf Adults and Its Nonaudiological Predictors: Psychological Distress, Coping Strategies, and Self-Esteem. Ear Hear.

Kokkinakis, K., Pak, N., 2014. Binaural advantages in users of bimodal and bilateral cochlear implant devices. J. Acoust. Soc. Am., 135(1), p.EL47-53.

Kompis, M., Dillier, N., 2001. Performance of an adaptive beamforming noise reduction scheme for hearing aid applications. II. Experimental verification of the predictions. J. Acoust. Soc. Am., 109(3), p.1134-1143.

Kong, Y., Carlyon, R.P., 2010. Temporal pitch perception at high rates in cochlear implants. J. Acoust. Soc. Am., 127(5), p.3114-3123.

Kong, Y., Stickney, G., Zeng, F., 2005. Speech and melody recognition in binaurally combined acoustic and electric hearing. J. Acoust. Soc. Am., 117(3), p.13511361.

Koning, R., Madhu, N., Wouters, J., 2015. Ideal time-frequency masking algorithms lead to different speech intelligibility and quality in normal-hearing and cochlear implant listeners. IEEE Trans. Biomed. Eng., 62(1), p.331-341.

Kraaijenga, V.J.C., Smit, A.L., Stegeman, I., Smilde, J.J.M., van Zanten, G.A., et al, 2015. Factors that influence outcomes in cochlear implantation in adults, based on patient related characteristics - a retrospective study. Clin. Otolaryngol., p.1-8.

Kramer, S.E., Kapteyn, T.S., Festen, J.M., Tobi, H., 1995. Factors in subjective hearing disability. Audiology, 34(6), p.311-20.

Kramer, S.E., Kapteyn, T.S., Festen, J.M., Tobi, H., 1996. The relationships between self-reported hearing disability and measures of auditory disability. Audiology, 35(5), p.277-87.

Kramer, S.E., Kapteyn, T.S., Kuik, D.J., Deeg, D.J.H., 2002. The Association of Hearing Impairment and Chronic Diseases with Psychosocial Health Status in Older Age. J. Aging Health, 14(1), p.122-137.

Kramer, S.E., Zekveld, A. a, Houtgast, T., 2009. Measuring cognitive factors in speech comprehension: the value of using the Text Reception Threshold test as a visual equivalent of the SRT test. Scand. J. Psychol., 50(5), p.507-15. 
Krueger, B., Joseph, G., Rost, U., Strauss-Schier, A., Lenarz, T., et al, 2008. Performance groups in adult cochlear implant users: speech perception results from 1984 until today. Otol. Neurotol., 29(4), p.509-12.

Landis, J.R., Koch, G.G., 1977. The Measurement of Observer Agreement for Categorical Data. Biometrics, 33(1), p.159.

Landsberger, D.M., Svrakic, M., Roland, J.T., Svirsky, M., 2015. The Relationship Between Insertion Angles, Default Frequency Allocations, and Spiral Ganglion Place Pitch in Cochlear Implants. Ear Hear., 36(5), p.e207-13.

Leek, M.R., 2001. Adaptive procedures in psychophysical research. Percept. Psychophys., 63(8), p.1279-1292.

Leigh, J.R., Moran, M., Hollow, R., Dowell, R.C., 2016. Evidence-based guidelines for recommending cochlear implantation for postlingually deafened adults. Int. J. Audiol., 2027, p.1-6.

Lenarz, T., Muller, L., Czerniejewska-Wolska, H., Varela, H.V., Dotú, C.O., et al, 2017. Patient-Related Benefits for Adults with Cochlear Implantation: A Multicultural Longitudinal Observational Study. Audiol. Neurotol., 22(2), p.61-73.

Lepage, B., Deguine, O., Taoui, S., Molinier, L., Costa, N., et al, 2019. Cochlear implantation as a treatment for singlesided deafness and asymmetric hearing loss: a randomized controlled evaluation of cost-utility. BMC Ear, Nose Throat Disord., 19(1), p.1-10.

Litovsky, R., 2015. Development of the Auditory System. In Handb Clin Neurol. pp. 55-72.

Litovsky, R.Y., Johnstone, P.M., Godar, S.P., 2006. Benefits of bilateral cochlear implants and/or hearing aids in children. Int. J. Audiol., 45, p.S78-91.

Little, R.J.A., Rubin, D.B., 2002. Statistical Analysis with Missing Data, New York, USA: John Wiley \& Sons, Inc.
Looi, V., McDermott, H., McKay, C., Hickson, L., 2007. Comparisons of quality ratings for music by cochlear implant and hearing aid users. Ear Hear., 28(2 Suppl), p.59S-61S.

van Loon, M.C., Smits, C., Smit, C.F., Hensen, E.F., Merkus, P., 2017. Cochlear Implantation in Adults With Asymmetric Hearing Loss. Otol. Neurotol., p.1.

Van Loon, M.C., Smits, C., Smit, C.F., Hensen, E.F., Merkus, P., 2017. Cochlear Implantation in Adults with Asymmetric Hearing Loss: Benefits of Bimodal Stimulation. Otol. Neurotol. 38(6), p.e100-e106.

Lotfi, Y., Hasanalifard, M., Moossavi, A., Bakhshi, E., Ajaloueyan, M., 2019. Binaural hearing advantages for children with bimodal fitting. Int. J. Pediatr. Otorhinolaryngol., 121(December 2018), p.58-63.

Luntz, M., Shpak, T., Weiss, H., 2005. Binauralbimodal hearing: concomitant use of a unilateral cochlear implant and a contralateral hearing aid. Acta Otolaryngol, 125(8), p.863-869.

Luntz, M., Yehudai, N., Shpak, T., 2007. Hearing progress and fluctuations in bimodalbinaural hearing users (unilateral cochlear implants and contralateral hearing aid). Acta Otolaryngol., 127(10), p.1045-1050.

Luo, X., Chang, Y.-P., Lin, C.-Y., Chang, R.Y., 2014. Contribution of bimodal hearing to lexical tone normalization in Mandarin-speaking cochlear implant users. Hear. Res., 312, p.1-8.

Luts, H., Eneman, K., Wouters, J., Schulte, M., Vormann, M., et al, 2010. Multicenter evaluation of signal enhancement algorithms for hearing aids. J. Acoust. Soc. Am., 127(3), p.1491-1505.

Mackenzie, E., Lutman, M.E., 2005. Speech Recognition and Comfort Using Hearing Instruments with Adaptive Directional Characteristics in Asymmetric Listening Conditions. Ear Hear., 26(6), p.669-679. 
Maes, I.H.L., Joore, M. a, Cima, R.F.F., Vlaeyen, J.W., Anteunis, L.J.C., 2011. Assessment of health state in patients with tinnitus: a comparison of the EQ-5D and HUI mark III. Ear Hear., 32(4), p.428-35.

McCormack, A., Fortnum, H., 2013. Why do people fitted with hearing aids not wear them? Int. J. Audiol., 52(5), p.3608.

McCreery, R.W., Venediktov, R.A., Coleman, J.J., Leech, H.M., 2012. An Evidence-Based Systematic Review of Directional Microphones and Digital Noise Reduction Hearing Aids in School-Age Children With Hearing Loss. Am. J. Audiol., 21(2), p.295-312.

McDermott, H.J., Sucher, C.M., 2006. Perceptual dissimilarities among acoustic stimuli and ipsilateral electric stimuli. Hear. Res., 218(1-2), p.81-88.

McRackan, T.R., Bauschard, M., Hatch, J.L., Franko-Tobin, E., Droghini, H.R., et al, 2018. Meta-analysis of quality-of-life improvement after cochlear implantation and associations with speech recognition abilities. Laryngoscope, 128(4), p.982-990.

McRackan, T.R., Hand, B.N., Velozo, C.A., Dubno, J.R., 2019. Association of Demographic and Hearing-Related Factors With Cochlear Implant-Related Quality of Life. JAMA Otolaryngol. Neck Surg., p.1-9.

Metz, C., 1978. Basic principles of ROC analysis. Semin. Nucl. Med., 8(4), p.283298.

Mishra, S., Lunner, T., Stenfelt, S., Rönnberg, J., Rudner, M., 2013. Seeing the talker's face supports executive processing of speech in steady state noise. Front. Syst. Neurosci., 7(November), p.96.

Mok, M., Galvin, K.L., Dowell, R.C., McKay, C.M., 2010. Speech perception benefit for children with a cochlear implant and a hearing aid in opposite ears and children with bilateral cochlear implants. Audiol. Neurootol., 15(1), p.44-56.
Mok, M., Grayden, D., Dowell, R.C., Lawrence, D., 2006. Speech perception for adults who use hearing aids in conjunction with cochlear implants in opposite ears. J Speech Lang Hear Res, 49(2), p.338-351.

Moore, B., Glasberg, B., 2007. Modeling binaural loudness. J. Acoust. Soc. Am., 121(3), p.1604-1612.

Morera, C., Cavalle, L., Manrique, M., Huarte, A., Angel, R., et al, 2012. Contralateral hearing aid use in cochlear implanted patients: Multicenter study of bimodal benefit. Acta Otolaryngol.

Morera, C., Manrique, M., Ramos, A., GarciaIbanez, L., Cavalle, L., et al, 2005. Advantages of binaural hearing provided through bimodal stimulation via a cochlear implant and a conventional hearing aid: a 6-month comparative study. Acta Otolaryngol, 125(6), p.596-606.

Morimoto, M., Sato, H., Kobayashi, M., 2004. Listening difficulty as a subjective measure for evaluation of speech transmission performance in public spaces. J. Acoust. Soc. Am., 116(3), p.1607-1613.

Most, T., Gaon-Sivan, G., Shpak, T., Luntz, M., 2012. Contribution of a contralateral hearing aid to perception of consonant voicing, intonation, and emotional state in adult cochlear implantees. J Deaf Stud Deaf Educ, 17(2), p.244-258.

Mulrow, C.D., 1990. Quality-of-Life Changes and Hearing Impairment. Ann. Intern. Med., 113(3), p.188.

Nachtegaal, J., Festen, J.M., Kramer, S.E., 2012. Hearing ability in working life and its relationship with sick leave and selfreported work productivity. Ear Hear.

Nelson, P.B., Jin, S.-H., 2004. Factors affecting speech understanding in gated interference: Cochlear implant users and normal-hearing listeners. J. Acoust. Soc. Am., 115(5), p.2286.

Neuman, A.C., Svirsky, M. a, 2013. Effect of Hearing Aid Bandwidth on Speech Recognition Performance of Listeners 
Using a Cochlear Implant and Contralateral Hearing Aid (Bimodal Hearing). Ear Hear., 34(5), p.553-561.

Neuman, A.C., Waltzman, S.B., Shapiro, W.H., Neukam, J.D., Zeman, A.M., et al, 2017. Self-Reported Usage , Functional Benefit, and Audiologic Characteristics of Cochlear Implant Patients Who Use a Contralateral Hearing Aid. Trends Hear., 21, p.1-14.

Neuman, A.C., Zeman, A., Neukam, J., Wang, B., Svirsky, M.A., 2018. The Effect of Hearing Aid Bandwidth and Configuration of Hearing Loss on Bimodal Speech Recognition in Cochlear Implant Users. Ear Hear., (1), p.1.

Ng, E.H.N., Rudner, M., Lunner, T., Pedersen, M.S., Rönnberg, J., 2013. Effects of noise and working memory capacity on memory processing of speech for hearing-aid users. Int. J. Audiol., 52(7), p.433-41.

NIDCD, 2016. Cochlear Implants. Fact Sheet No. 00-4798, p.2. Available at: https://www.nidcd.nih.gov/sites/defa ult/files/Documents/health/hearing/C ochlearImplants.pdf.

Noble, J., Gifford, R., Hedley-Williams, A., Dawant, B., Labadie, R., 2014. Clinical Evaluation of an Image-Guided Cochlear Implant Programming Strategy. Audiol. Neurotol., 19, p.400411.

Noble, W., Gatehouse, S., 2006. Effects of bilateral versus unilateral hearing aid fitting on abilities measured by the Speech, Spatial, and Qualities of Hearing scale (SSQ). Int. J. Audiol., 45(3), p.172-181.

Noble, W., Jensen, N.S., Naylor, G., Bhullar, N., Akeroyd, M. a, 2013. A short form of the Speech, Spatial and Qualities of Hearing scale suitable for clinical use: the SSQ12. Int. J. Audiol., 52(6), p.409-12.

Noble, W., Tyler, R., Dunn, C., Bhullar, N., 2008a. Hearing handicap ratings among different profiles of adult cochlear implant users. Ear Hear, 29(1), p.112-120.
Noble, W., Tyler, R., Dunn, C., Bhullar, N., 2008b. Unilateral and bilateral cochlear implants and the implant-plus-hearingaid profile: comparing self-assessed and measured abilities. Int J Audiol, $47(8)$, p.505-514.

Oetting, D., Hohmann, V., Appell, J.-E., Kollmeier, B., Ewert, S.D., 2017. Restoring Perceived Loudness for Listeners With Hearing Loss. Ear Hear., p.1.

Offeciers, E., Morera, C., Müller, J., Huarte, A., Shallop, J., et al, 2005. International consensus on bilateral cochlear implants and bimodal stimulation. Acta Otolaryngol., 125(9), p.918-9.

Ohlenforst, B., Zekveld, A.A., Jansma, E.P., Wang, Y., Naylor, G., et al, 2017. Effects of Hearing Impairment and Hearing Aid Amplification on Listening Effort: A Systematic Review. Ear Hear., 38(3), p.267-281.

Olson, A.D., Shinn, J.B., 2008. A systematic review to determine the effectiveness of using amplification in conjunction with cochlear implantation. J. Am. Acad. Audiol., 19(9), p.657-671.

Olusanya, B.O., Neumann, K.J., Saunders, J.E., 2014. The global burden of disabling hearing impairment: a call to action. Bull. World Heal. Organ.

OPCI, 2018. Aantal implantaties in Nederland. Available at: http://www.opciweb.nl/ci-centra/cicentra-in-nederland/aantalimplantaties-in-nederland/.

Oxenham, A.J., Bernstein, J.G.W., Penagos, H., 2004. Correct tonotopic representation is necessary for complex pitch perception. Proc. Natl. Acad. Sci. U. S. A., 101(5), p.1421-1425.

Park, L.R., Teagle, H.F.B., Buss, E., Roush, P. a, Buchman, C. a, 2012. Effects of frequency compression hearing aids for unilaterally implanted children with acoustically amplified residual hearing in the nonimplanted ear. Ear Hear., 33(4), p.e1-e12. 
Parkinson, A.J., Arcaroli, J., Staller, S.J., Arndt, P.L., Cosgriff, A., et al, 2002. The nucleus 24 contour cochlear implant system: adult clinical trial results. Ear Hear., 23(1 Suppl), p.41S-48S

Peretz, I., Ayotte, J., Zatorre, R.J., Mehler, J., Ahad, P., et al, 2002. Congenital Amusia: A disorder of fine-grained pitch discrimination. Neuron, 33(2), p.185-191.

Peterson, P., Zurek, P., 1987. Multimicrophone adaptive beamforming for reduction in hearing aids. J. Rehabil. Res. Dev, 24(4), p.103-110.

Pieper, S., Lorensen, B., Schroeder, W., Kikinis, R., 2006. The NA-MIC Kit: ITK, VTK, Pipelines, Grids and 3D Slicer as An Open Platform for the Medical Image Computing Community. Biomed. Imaging Nano to Macro, 2006. 3rd IEEE Int. Symp., p.698-701.

Pisoni, D.B., Kronenberger, W.G., Harris, M.S., Moberly, A.C., 2017. Three challenges for future research on cochlear implants. World J. Otorhinolaryngol. Head Neck Surg., 3(4), p.240-254.

Plomp, R., 1978. Auditory handicap of hearing impairment and the limited benefit of hearing aids. J. Acoust. Soc. Am., 63(2), p.533-549.

Polonenko, M.J., Papsin, B.C., Gordon, K.A., 2018. Delayed access to bilateral input alters cortical organization in children with asymmetric hearing. Neurolmage Clin., 17(July 2017), p.415-425.

Potts, L.G., Skinner, M.W., Litovsky, R.A., Strube, M.J., Kuk, F., 2009. Recognition and localization of speech by adult cochlear implant recipients wearing a digital hearing aid in the nonimplanted ear (bimodal hearing). J Am Acad Audiol, 20(6), p.353-373.

Preminger, J.E., Van Tasell, D.J., 1995a. Measurement of speech quality as a tool to optimize the fitting of a hearing aid. J. Speech Hear. Res., 38(3), p.72636.
Preminger, J.E., Van Tasell, D.J., 1995b. Quantifying the relation between speech quality and speech intelligibility. J Speech Hear Res, 38(3), p.714-725.

Punch, J.L., Hitt, R., Smith, S.W., 2019. Hearing loss and quality of life. J. Commun. Disord., 78(January), p.33-45.

Qin, M., Oxenham, A., 2003. Effects of simulated cochlear-implant processing on speech reception in fluctuating maskers. J. Acoust. Soc. Am., 114(1), p.446-454.

Qin, M.K., Oxenham, A.J., 2006. Effects of introducing unprocessed lowfrequency information on the reception of envelope-vocoder processed speech. J. Acoust. Soc. Am., 119(4), p.2417-26.

De Raeve, L., 2015. De prevalentie van cochleaire implantaten in België en Nederland: wat weten we en wat kunnen we verwachten. In 10th EuroCIU symposium on 9-10 April 2015 in Antwerp.

Ramakers, G.G.J., Smulders, Y.E., van Zon, A., Kraaijenga, V.J.C., Stegeman, I., et al, 2016. Agreement between health utility instruments in cochlear implantation. Clin. Otolaryngol., 41(6), p.737-743.

Reiss, L. a J., Ito, R. a, Eggleston, J.L., Liao, S., Becker, J.J., et al, 2014a. Pitch Adaptation Patterns in Bimodal Cochlear Implant Users: Over Time and After Experience. Ear Hear., p.1-12.

Reiss, L. a J., Turner, C.W., Karsten, S. a., Gantz, B.J., 2014b. Plasticity in human pitch perception induced by tonotopically mismatched electro-acoustic stimulation. Neuroscience, 256, p.4352 .

Ricketts, T. a, Henry, P.P., Hornsby, B.W.Y., 2005. Application of frequency importance functions to directivity for prediction of benefit in uniform fields. Ear Hear., 26(5), p.473-86.

Ricketts, T. a, Picou, E.M., 2013. Speech recognition for bilaterally asymmetric and symmetric hearing aid microphone 
modes in simulated classroom environments. Ear Hear., 34(5), p.601609.

Ricketts, T., Mueller, H., 1999. Making sense of directional microphone hearing aids. Am. J. Audiol., 8(4), p.117-127.

Rönnberg, J., Lunner, T., Zekveld, A., Sörqvist, P., Danielsson, H., et al, 2013. The Ease of Language Understanding (ELU) model: theoretical, empirical, and clinical advances. Front. Syst. Neurosci., $7(31)$.

Rönnberg, J., Rudner, M., Lunner, T., Zekveld, A.A., 2010. When cognition kicks in: working memory and speech understanding in noise. Noise Health, 12(49), p.263-9.

Rönnberg, N., Rudner, M., Lunner, T., Stenfelt, S., 2014. Memory performance on the Auditory Inference Span Test is independent of background noise type for young adults with normal hearing at high speech intelligibility. Front. Psychol., 5, p.1-11.

le Roux, T., Vinck, B., Butler, I., Louw, L., Nauta, L., et al, 2017. Predictors of healthrelated quality of life in adult cochlear implant recipients in South Africa. Int. J. Audiol.

Rubin, D.B., 1987. Multiple Imputation for Nonresponse in Surveys D. B. Rubin, ed., New York, USA: John Wiley \& Sons, Inc.

Rudner, M., Lunner, T., 2014. Cognitive spare capacity and speech communication: A narrative overview. Biomed Res. Int., 2014.

Rudner, M., Lunner, T., Behrens, T., Thorén, E.S., Rönnberg, J., 2012. Working Memory Capacity May Influence Perceived Effort during Aided Speech Recognition in Noise. J. Am. Acad. Audiol., 23(8), p.577-589.

Rudner, M., Rönnberg, J., Lunner, T., 2011. Working memory supports listening in noise for persons with hearing impairment. J. Am. Acad. Audiol., 22(3), p.156-67.
Runge, C.L., Du, F., Hu, Y., 2018. Improved speech perception in cochlear implant users with interleaved high-rate pulse trains. Otol. Neurotol., 39(5), p.e319e324.

Sarampalis, A., Kalluri, S., Edwards, B., Hafter, E., 2009. Objective measures of listening effort: effects of background noise and noise reduction. J. Speech. Lang. Hear. Res., 52(5), p.1230-1240.

Schafer, E.C., Amlani, A.M., Paiva, D., Nozari, L., Verret, S., 2011. A meta-analysis to compare speech recognition in noise with bilateral cochlear implants and bimodal stimulation. Int. J. Audiol., 50(12), p.871-880.

Schatzer, R., Vermeire, K., Visser, D., Krenmayr, A., 2014. Electric-acoustic pitch comparisons in single-sided-deaf cochlear implant users: Frequencyplace functions and rate pitch. Hear. Res., 309, p.26-35.

Scherf, F.W. a C., Arnold, L.P., 2014. Exploring the clinical approach to the bimodal fitting of hearing aids and cochlear implants: results of an international survey. Acta Otolaryngol., 134(11), p.1151-1157.

Schulte, M., Wagener, K., Vormann, M., Dillier, N., Büchner, M., 2008. D-7-4: Report Tests for listening effort. In M. Shulte, ed. Hearing in the Communiction Society. pp. 1-24.

Schulte, Meis, Wagener, 2007. Listening Effort and Speech Intelligibility. , (Presented at the 8th EFAS congress of the European Fedearion of Audiological Societies, Heidelberg, Germany, June 69, 2007). Available at: http://www.uzh.ch/orl/dga2007/prog ram/scientificprogram/Schulte_M._et_ al.pdf.

Sheffield, S.W., Gifford, R.H., 2014. The Benefits of Bimodal Hearing: Effect of Frequency Region and Acoustic Bandwidth. Audiol. Neurootol., 19(3), p.151-163.

Shpak, T., Most, T., Luntz, M., 2013. Fundamental frequency information for speech recognition via bimodal 
stimulation: cochlear implant in one ear and hearing aid in the other. Ear Hear., 35, p.97-109.

Shrout, P.E., Fleiss, J.L., 1979. Intraclass correlations: Uses in assessing rater reliability. Psychol. Bull., 86(2), p.420428.

Siburt, H.W., Holmes, A.E., 2015. Bimodal Programming: A Survey of Current Clinical Practice. Am. J. Audiol., 24(2), p.243.

Siciliano, C.M., Faulkner, A., Rosen, S., Mair, K., 2010. Resistance to learning binaurally mismatched frequency-to-place maps: implications for bilateral stimulation with cochlear implants. J. Acoust. Soc. Am., 127(3), p.1645-1660.

Silman, S., Silverman, C.A., Emmer, M.B., Gelfand, S.A., 1993. Effects of prolonged lack of amplification on speechrecognition performance: Preliminary findings. J. Rehabil. Res. Dev., 30(3), p.326-332.

Skinner, M., 1982. Amplification bandwidth and speech intelligibility for two listeners with sensorineural hearing loss. Audiol. J. Audit. Commun., 21(3), p.251-268.

Slawson, W., 1985. Sound color, Berkeley, California: University of California Press.

Smits, C., Festen, J., 2011. The interpretation of speech reception threshold data in normal-hearing and hearing-impaired listeners: Steady-state noise. J. Acoust. Soc. Am., 130(5), p.2987-2998.

Smulders, Y.E., Van Zon, A., Stegeman, I., Van Zanten, G.A., Rinia, A.B., et al, 2016. Cost-utility of bilateral versus unilateral cochlear implantation in adults: A randomized controlled trial. Otol. Neurotol., 37(1), p.38-45.

Sockalingam, R., Beilin, J., Beck, D., 2009. Sound Quality Considerations of Hearing Instruments. Hear. Rev., 16(3), p.22-28.
Soede, W., 1993. Assessment of a directional microphone array for hearing-impaired listeners. J. Acoust. Soc. Am., 94(2), p.799.

Spriet, A., Van Deun, L., Eftaxiadis, K., Laneau, J., Moonen, M., et al, 2007. Speech understanding in background noise with the two-microphone adaptive beamformer BEAM in the Nucleus Freedom Cochlear Implant System. Ear Hear., 28(1), p.62-72.

Staehelin, K., Bertoli, S., Probst, R., Schindler, C., Dratva, J., et al, 2011. Gender and Hearing Aids: Patterns of Use and Determinants of Nonregular Use. Ear Hear., 32(6), p.e26-e37.

Stakhovskaya, O., Sridhar, D., Bonham, B.H., Leake, P.A., 2007. Frequency Map for the Human Cochlear Spiral Ganglion: Implications for Cochlear Implants. $J$. Assoc. Res. Otolaryngol., 8(2), p.220233.

Stephens, S., 1976. The Input for a Damaged Cochlea - A Brief Review. Br. J. Audiol., 10(4), p.97-101.

Sucher, C.M., McDermott, H.J., 2009. Bimodal stimulation: benefits for music perception and sound quality. Cochlear Implants Int., 10 Suppl 1, p.96-9.

Suelzle, D., Parsa, V., Falk, T., 2013. On a reference-free speech quality estimator for hearing aids. J. Acoust. Soc. Am., 133(5), p.EL412-418.

Summerfield, Q., Barton, G.R., Toner, J., McAnallen, C., Proops, D., et al, 2006. Self-reported benefits from successive bilateral cochlear implantation in postlingually deafened adults: randomised controlled trial. Int. J. Audiol., 45 Suppl 1(Supplement 1), p.S99-107.

Sung, G.S., Sung, R.J., Angelelli, R.M., 1975. Directional microphone in hearing aids. Effects on speech discrimination in noise. Arch. Otolaryngol., 101(5), p.316-319.

Syms, C., Wickesberg, J., Kubo, T., Takahashi, Y., Iwaki, T., 2002. Concurrent use of cochlear implants and hearing aids. In T. Kubo, Y. Takahashi, \& T. Iwaki, eds. 
Cochlear Implants-An Update. The Hague: Kugler Publications, pp. 535539.

Theelen-van den Hoek, F.L., Houben, R., Dreschler, W.A., 2014. Investigation into the applicability and optimization of the Dutch matrix sentence test for use with cochlear implant users. Int. J. Audiol., 53(11), p.817-28.

Todd, N.W., Ball, T.I., 2004. Interobserver agreement of coiling of Med-El cochlear implant: plain x-ray studies. Otol. Neurotol., 25(3), p.271-274.

Treutwein, B., 1995. Adaptive psychophysical procedures. Vision Res., 35(17), p.2503-2522.

Turner, C., 2008. Integration of acoustic and electrical hearing. J. Rehabil. Res. Dev., 45(5), p.769-778.

Tyler, R.S., Parkinson, A.J., Wilson, B.S., Witt, S., Preece, J.P., et al, 2002. Patients utilizing a hearing aid and a cochlear implant: speech perception and localization. Ear Hear., 23(2), p.98-105.

Vaerenberg, B., Smits, C., De Ceulaer, G., Zir, E., Harman, S., et al, 2014. Cochlear implant programming: A global survey on the state of the art. Sci. World J., 2014(August 2013).

Vermeire, K., Nobbe, A., Schleich, P., Nopp, P., Voormolen, M.H., et al, 2008. Neural tonotopy in cochlear implants: An evaluation in unilateral cochlear implant patients with unilateral deafness and tinnitus. Hear. Res., 245(1-2), p.98-106.

Vermeire, K., Schatzer, R., Punte, a K., Van de Heyning, P., Voormolen, M., et al, 2015. A frequency-place map for electrical stimulation in cochlear implants: Change over time. Hear. Res., (Epub 2015 Apr 1).

Veugen, L.C.E., Chalupper, J., Snik, A.F.M., van Opstal, A.J., Mens, L.H.M., 2016. Frequency-dependent loudness balancing in bimodal cochlear implant users. Acta Otolaryngol., 6489(March), p.1-7.
Veugen, L.C.E., Chalupper, J., Snik, A.F.M., van Opstal, A.J., Mens, L.H.M., 2015. Matching Automatic Gain Control Across Devices in Bimodal Cochlear Implant Users. Ear Hear., (epub ahead of print).

Vroegop, J.L., Dingemanse, J.G., Homans, N.C., Goedegebure, A., 2017. Evaluation of a wireless remote microphone in bimodal cochlear implant recipients. Int. J. Audiol., 0(0), p.1-7.

Vroegop, J.L., Goedegebure, A., van der Schroeff, M.P., 2018a. How to Optimally Fit a Hearing Aid for Bimodal Cochlear Implant Users. Ear Hear., p.1.

Vroegop, J.L., Homans, N.C., Schroeff, M.P. Van Der, Goedegebure, A., 2018b. Comparing Two Hearing Aid Fitting Algorithms for Bimodal Cochlear Implant Users. Ear Hear., p.1-9.

Wallhäusser-Franke, E., Balkenhol, T., Hetjens, S., Rotter, N., Servais, J.J., 2018. Patient benefit following Bimodal CI-provision: Self-reported Abilities vs. hearing status. Front. Neurol., 9(SEP), p.1-13.

Waltzman, S.B., Niparko, J.K., Fisher, S.G., Cohen, N.L., 1995. Predictors of postoperative performance with cochlear implants. Ann. Otol. Rhinol. Laryngol., 104(4 II SUPPL.), p.15-18.

Warren, S., Dunbar, M., 2018. Bimodal Hearing in Individuals with Severe-to-Profound Hearing Loss: Benefits, Challenges, and Management. Semin. Hear., 39(4), p.405-413.

Weissgerber, T., Rader, T., Baumann, U., 2015. Impact of a Moving Noise Masker on Speech Perception in Cochlear Implant Users. PLoS One, 10(5), p.e0126133.

Wilson, B.S., Dorman, M.F., 2008. Cochlear implants: current designs and future possibilities. J. Rehabil. Res. Dev., 45(5), p.695-730.

Winn, M., Edwards, J., 2013. The impact of spectral resolution on listening effort revealed by pupil dilation. J. Acoust. Soc. Am., 134(5), p.4233-. 
Wong, L.L.N., 2003. Hearing Aid Satisfaction: What Does Research from the Past 20 Years Say? Trends Amplif., 7(4), p.117161.

World Health Organisation, 2018. Deafness and hearing loss. Fact Sheet. Available at: http://www.who.int/en/newsroom/fact-sheets/detail/deafness-andhearing-loss.

World Health Organisation, 1980. International Classification of impairments, disabilites and handicaps (ICIDH). , (May 1976).

Wouters, J., Vanden Berghe, J., 2001. Speech Recognition in Noise for Cochlear Implantees with a Two-Microphone Monaural Adaptive Noise Reduction System. Ear Hear., 22(5), p.420-430.

Wu, Y.-H., Aksan, N., Rizzo, M., Stangl, E., Zhang, X., et al, 2014. Measuring Listening Effort: Driving Simulator Versus Simple Dual-Task Paradigm. Ear Hear., 35(6), p.623-632.

Xu, J., Xu, S., Cohen, L., Clark, G., 2000. Cochlear view: postoperative radiography for cochlear implantation. Otol. Neurotol., 21(1), p.49-56.

Yamaguchi, C.T., Goffi-Gomez, M.V.S., 2013. Prevalence of Contralateral Hearing Aid Use in Adults with Cochlear Implants . Int. Arch. Otorhinolaryngol. , 17, p.370-374.

Yawn, R.J., O'Connell, B.P., Dwyer, R.T., Sunderhaus, L.W., Reynolds, S., et al, 2018. Bilateral Cochlear Implantation Versus Bimodal Hearing in Patients With Functional Residual Hearing. Otol. Neurotol., 39(4), p.422-427.

Yehudai, N., Shpak, T., Most, T., Luntz, M., 2013. Functional Status of Hearing Aids in Bilateral-Bimodal Users. Otol. Neurotol., Epub(Ahead of print).

Yehudai, N., Shpak, T., Most, T., Luntz, M., 2012. Natural history of contralateral residual hearing in unilateral cochlear implant users - long-term findings. Acta Otolaryngol., 132(10), p.1073-1076.
Yoon, Y.-S., Shin, Y.-R., Gho, J.-S., Fu, Q.-J., 2015. Bimodal benefit depends on the performance difference between a cochlear implant and a hearing aid. Cochlear Implants Int., 16(3), p.159167.

Yoon, Y., Li, Y., Fu, Q.-J., 2012a. Speech recognition and acoustic features in combined electric and acoustic stimulation. J. speech, Lang. Hear. Res., 55(1), p.105-124.

Yoon, Y., Shin, Y.-R., Fu, Q.-J., 2012b. Clinical selection criteria for a second cochlear implant for bimodal listeners. Otol. Neurotol., 33(7), p.1161-1168.

Youden, W., 1950. Index for rating diagnostic tests. Cancer, 3(1), p.32-35.

Zekveld, A.A., Kramer, S.E., Festen, J.M., 2010. Pupil response as an indication of effortful listening: the influence of sentence intelligibility. Ear Hear., 31(4), p.480-490.

Zhang, T., Dorman, M.F., Spahr, A.J., 2010. Information from the voice fundamental frequency (F0) region accounts for the majority of the benefit when acoustic stimulation is added to electric stimulation. Ear Hear., 31(1), p.63-69.

Zhang, T., Spahr, A.J., Dorman, M.F., Saoji, A., 2013. Relationship between auditory function of nonimplanted ears and bimodal benefit. Ear Hear., 34(2), p.133-141.

Zhou, N., Xu, L., Lee, C.-Y., 2010. The effects of frequency-place shift on consonant confusion in cochlear implant simulations. J. Acoust. Soc. Am., 128(1), p.401-409.

Zirn, S., Polterauer, D., Keller, S., Hemmert, W., 2016. The effect of fluctuating maskers on speech understanding of highperforming cochlear implant users. Int. J. Audiol., 2027(February), p.1-10.

Zurek, P.M., 1993. Binaural advantages and directional effects in speech intelligibility. In G. Studebaker \& I. Hochberg, eds. Acoustical Factors Affecting Hearing Aid Performance. 
Boston, MA: Allyn \& Bacon, pp. 255276.

Zweig, M., Campbell, G., 1993. Receiveroperating characteristic (ROC) plots: a fundamental evaluation tool in clinical medicine. Clin. Chem., 39(4), p.561577.

Zwolan, T., Kileny, P.R., Smith, S., Mills, D., Koch, D., et al, 2001. Adult cochlear implant patient performance with evolving electrode technology. Otol. Neurotol., 22(6), p.844-9. 


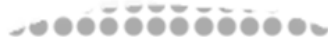

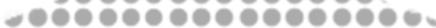

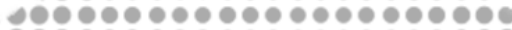

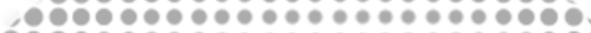

,

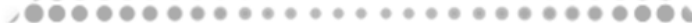

,

운,



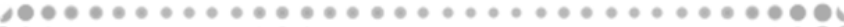

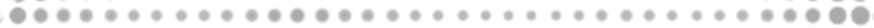

๑

เ

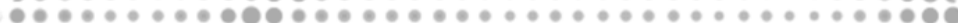

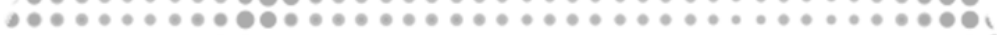

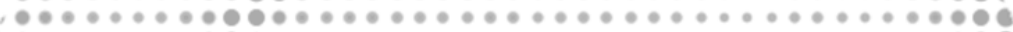

ㄴ․

,

$\circ$

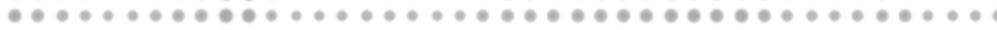

,

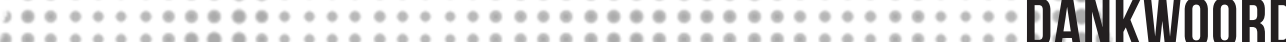

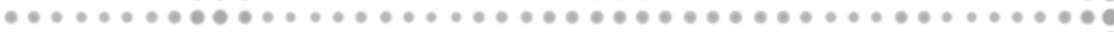

…

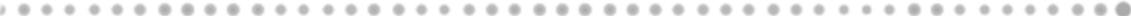

,

, * +

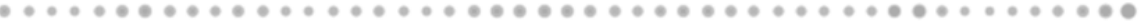

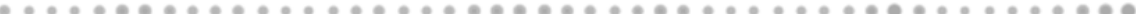

…

․

‥

… -

,

,

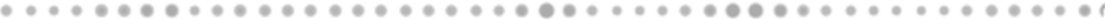

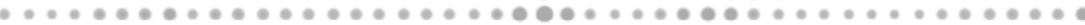

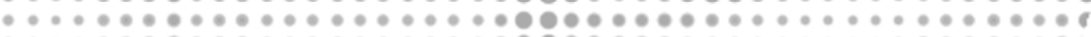

.

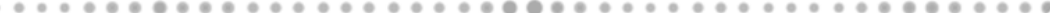

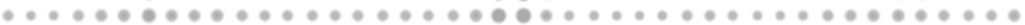

․

$\circ$

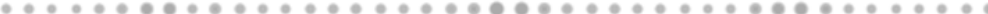

, + เ

$\circ \cdots$

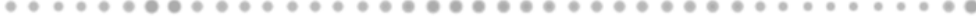

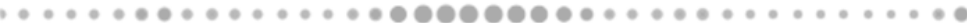

...... *.......

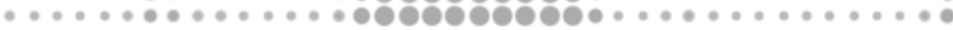

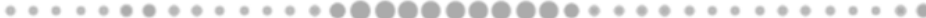

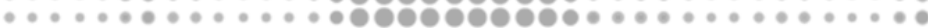

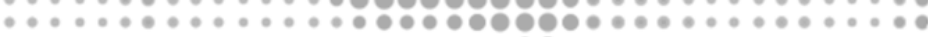

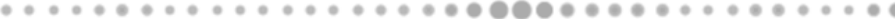

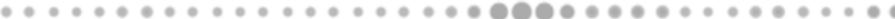

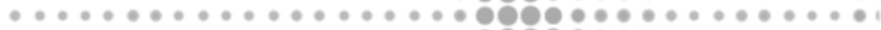

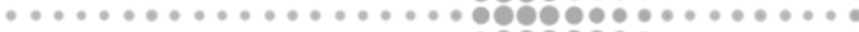

*..............

.................. -

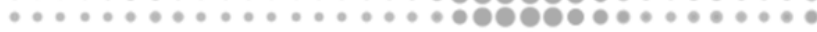

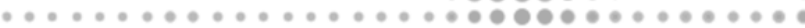

$\cdots \ldots$

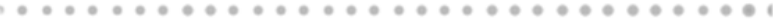

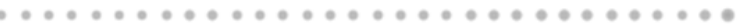

$\cdots \cdots$

एक

, ...........................

…

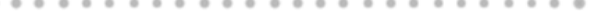

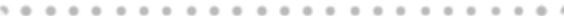

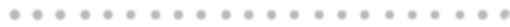

,

(2.

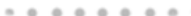





\title{
DANKWOORD
}

\author{
Ik heb gezocht, gevonden en verloren \\ en weer opnieuw gezocht totdat ik het weer vond \\ een mens wordt zoveel meer dan eens geboren \\ en voor wie zoekt is op 'n dag de cirkel rond \\ -TOON HERMANS-
}

Een proefschrift is een zoektocht. Een reis vol avonturen. Dit dankwoord is het laatste station. Want zonder alle tussenstops en medereizigers was ik nooit tot hier gekomen:

\section{$\checkmark$ IEMANDPLANT EEN IDEE}

Jezelf als promovendus zien, dat moet je leren. Iemand zag dat wel zitten, maar toen paste het niet op mijn pad. Het idee was gezaaid en broeide verder. Prof. Van Wieringen en Prof. Wouters. Bedankt!

\section{$\checkmark$ JE BAGAGE STAAT KLAAR}

Onvoorwaardelijke steun en familiale gezelligheid maakt een mens sterk. Een warme thuis geeft je fundamenten en lokt je telkens weer terug. Moeke, Vake, Joren en Dorien. Bedankt!

$\checkmark$ DE HORIZON LONKT NAAR AVONTUUR

Het audiologisch panorama onthult zich in het hartje van Nederland. Met directe tong en een groot hart voor de patiënt de praktijk leren kennen. Collega's van het Audiologisch Centrum AMC. Bedankt!

\section{$\checkmark \quad$ JE KRIJGT EEN LAATSTE DUWTJE}

Een gelegenheid biedt zich aan. Twijfels ontstaan, want je zit eigenlijk goed. Toch zou een avontuur zoveel kunnen betekenen voor de kansen die anderen in jou zien. Patrick, Hiske, Sabine. Bedankt!

\section{$\checkmark \quad$ JEDEELNAME WORDT AANVAARD}

Er stond een reis geboekt die nog uitgestippeld moest worden. Een avontuur waarvoor dé KNO implant chirurg uit het zuiden me geschikt vond en me de start heeft gegeven. Later hebben onze wegen zich gescheiden. Maar iedereen ziet, het gaat je goed. Prof. Stokroos. Bedankt!

\section{JE STIPPELT EEN PLAN UIT}

Een plan voor een route die je niet kent. Dan heb je een goede gids nodig. Eentje die altijd beschikbaar is en je door de bomen het bos weer laat zien. 
Zodat het geheel waaruit de delen bestaan groeit en vruchten afwerpt. Erwin. Bedankt!

$\checkmark$ EEN TREINKAARTJE VOOR ENKELE JAREN

Zonder vervoer en middelen kom je niet vooruit. Je zoekt je bestemmingen uit en kijkt wat mogelijk is. Over dalen en toppen heen, soms met vertraging, maar vaak ook in een hogere versnelling. Tips en ideeën kunnen licht werpen op nieuwe paden. Advanced Bionics, Sepp, Martina, Kristof. Bedankt!

\section{$\checkmark \quad$ EEN PRAATJE MET DE LOKALE BEVOLKING}

Je welkom voelen tussen de locals. Een gezellige babbel tussendoor. Een hart voor hetzelfde werkveld. Het is nooit te veel als je om hulp vraagt. Autoritjes worden leuker, uitstapjes gezelliger, je beroepsgroep wordt uitgedragen. Collega's van het Audiologisch Centrum MUMC+, de afdeling KNO. Lucien, Jan, Winde, Els, Joke, Nadia. Bedankt!

\section{$\checkmark$ SAMEN REIZEN SMEEDT BANDEN}

Van de ene plek naar de andere, zoekend naar die van jou, vind je reisgenoten. Samen dingen uitspitten, frustraties en overwinningen delen. We werden een onderzoeksgroep. Zowel tijdens als na de werkuren vonden we elkaar. Anouk, Marc, Remo. Bedankt!

De ene zwaaiden we in glorie uit, de volgende stond alweer op de stoep klaar. Een schouderklopje hier, een koffietje daar, lunchtijd of niet, we steunen elkaar! Jasper, Michel, Tim, Floor, Gusta, Joost, Marly, Frans, Kiki, Lars en alle studenten. Bedankt!

Extra input kan je gebruiken, zeker als het je eigen bagage net te buiten gaat. Samen zoeken en vinden, dat doet je sterker in je schoenen staan. Mickey, Miranda. Bedankt!

\section{$\checkmark$ PASSANTEN WIJZEN JE DE WEG}

De reis der wetenschap leer je uit de wereld om je heen. Enkel door alle input, enthousiaste reacties, tijd, motivatie en ieder antwoord samen kon deze output ontstaan. Jullie zijn de bron van mijn reis. Alle proefpersonen die hun steentje bijdroegen aan dit onderzoek. Bedankt!

\section{$\checkmark$ EEN LEVENSREIS IS NIET OVERLEVEN MAAR BELEVEN}

Jij de zon en ik de maan, zo samen door het leven gaan. Elke avond twee armen om in thuis te komen. Elkaar pauzeren, maar ook uitdagen en beleven. Jouw ruggensteun had ik nodig om mijn reis over pieken en door dalen heen voort te zetten. Gerd. Bedankt! 
De reis komt even on hold te staan voor een ander groot avontuur. Je leven wordt nooit meer hetzelfde, gelukkig maar. Elke dag twee guitige oogjes die je vol pit mama noemen. Jij bent ons kersje op de taart. Nog eventjes geduld tot nog een paar oogjes jou grote zus zullen noemen. Marlo. Bedankt!

\section{EEN ZIJSPOOR VERKENNEN}

Een andere wereld met vraag om hulp. Ondanks dat er maar een druppel valt op een hete plaat, kennen die paar ogen zoveel dankbaarheid. Een onbeschrijflijke ervaring. Een team met toewijding. De hoop op een wederkerend avontuur. Echter blijkt de toegang een uitdaging op zich. Lombok. Jantine, Holis. Jan-Wouter \& Toon, Marie-José, Auke \& Koen. Bedankt!

\section{$\checkmark$ JE ACHTERBAN STEUNT JE VANAF DE ZIJLIJN}

Kaartjes vanuit het thuisfront. Hoe gaat het? Hoe verloopt je avontuur? Als iemand het kan, dan ben jij dat! Een etentje hier, een babbeltje daar. Wie weet krijgen we het toch voor elkaar. Vriendinnen uit Klasje 6.1, Kemmel 2, meiden LAW. Familie. Schoonfamilie. Ruben \& Katrien, Koen \& Karlien. Bedankt!

\section{EEN NIEUWE BESTEMMING DIENT ZICH AAN}

Denkkronkels uitspitten en boeken doorwurmen daagt je uit, maar zorgen voor elkaar zit je in het bloed. Je trekt terug naar waar je motivatie ligt. Een frisse start, een warm welkom. Ik heb mijn plekje daar gevonden. Collega's Audiologie \& Communicatie, Adelante, Hoensbroek. Bedankt!

\section{EEN LAATSTE SPRINTJE}

Het licht aan het einde van de tunnel is in zicht. De laatste loodjes. Een nieuwe blik, een laatste duwtje. Iemand die je brengt tot het doel van je reis. Prof. Kingma. Bedankt!

\section{$\checkmark$ MEMOIRES}

Zo'n reis maak je maar één keer in je leven en je legt het resultaat voor altijd vast. Zie hier dit boekje. Anouk, Rosse \& Josse. Bedankt!

\section{$\checkmark$ HET AVONTUUR WORDT VERVOLGD}

Ideeën groeien en bloeien. Zeker als de reismicrobe wordt gedeeld. Spinsels krijgen vorm. Wat zal de volgende bestemming zijn? Een nieuwe reisroute wordt uitgestippeld. De fakkel gaat branden en wordt overgedragen. Marc, Joke, Erwin en Lars. Bedankt! 



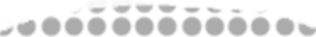

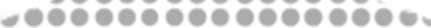

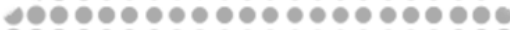

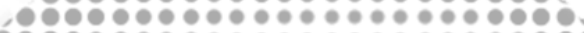
,

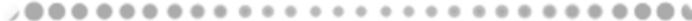

,

○우

ر우

운

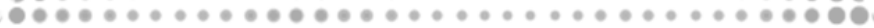

ค.

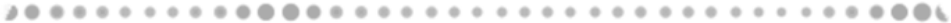

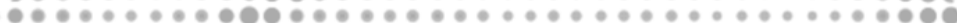

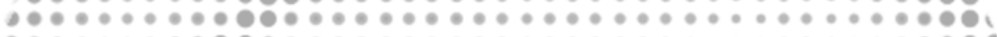

,

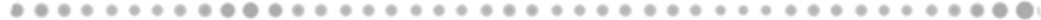

,

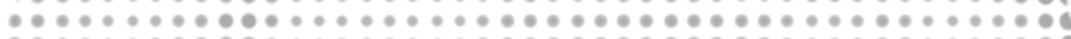

(5)

-

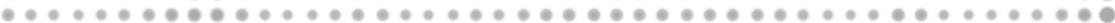

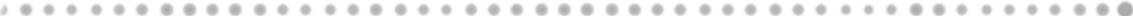

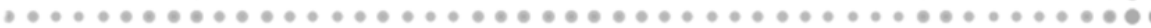

, * +

…

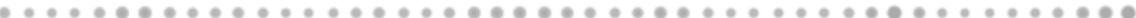

, . +

․

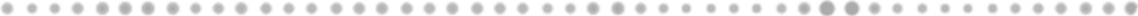

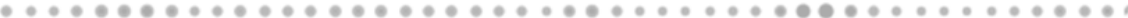

,

,

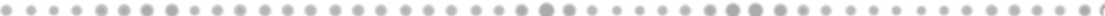

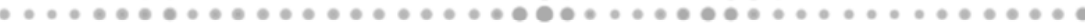

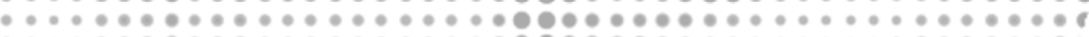

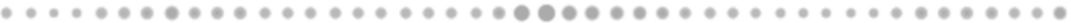

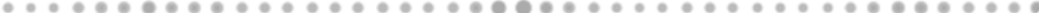

. . .

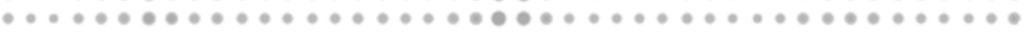

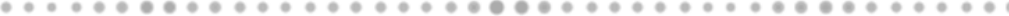

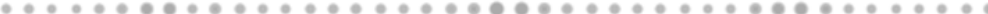

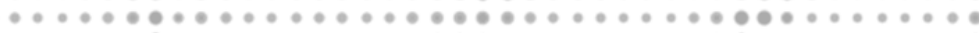

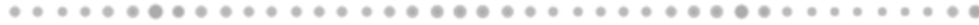

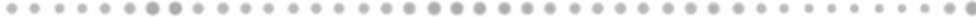

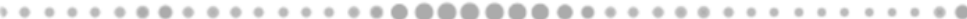

..............

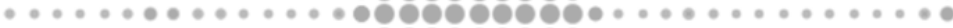

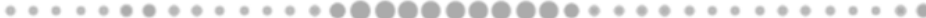

. . . * *......

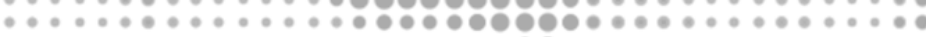

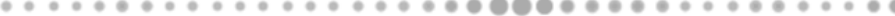

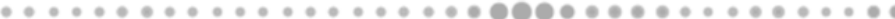

.... ..............

… *............

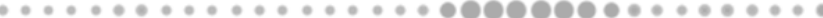

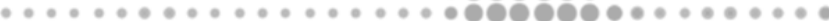

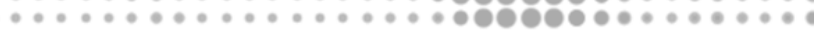

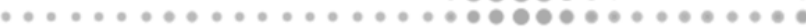

ह.

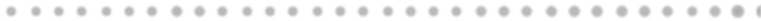

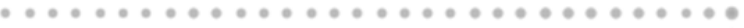

$\cdots \cdots$

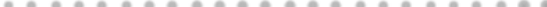

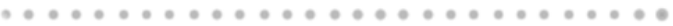

$\cdots \cdots$

-

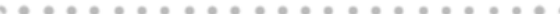

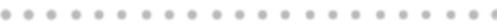

\%

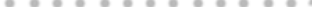

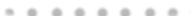





\section{CURRICULUM VITAE}

Elke (Maria Johanna) Devocht was born on the 11 th of March in 1987 in Turnhout (Belgium), where she grew up as the oldest sibling of three in a warm and caring family. She went to pre-school, primary school and secondary school at the Heilig Graf Institute in Turnhout (Belgium). In 2005 she completed her general secondary studies in Latin-Mathematics with success and started her studies in Speech Therapy and Audiological Sciences at the Catholic University of Leuven (KUL, Belgium). She obtained her Bachelor degree and consequently her Master degree summa cum laude in 2008 and 2009. During her Masters she did an internship in the Audiological Centre at the Amsterdam Medical Centre (AMC, Amsterdam, the Netherlands) and wrote a joined thesis on 'Localisation and spatial speech understanding in noise in cochlear implanted children' to complete her specialisation in Audiological Sciences. In 2010 she concluded her studies at the KUL with a post graduate degree in Audiology and Hearing Aid Fitting. In 2009 she already had returned to the Audiological Centre AMC to start her working career as a Master Audiologist. During three years she lived in Weesp (the Netherlands) and gained valuable clinical experience with cochlear implant and hearing aid fitting within the adult hearing rehabilitation team at AMC. In 2012 she accepted the challenge to start as a PhD candidate at the ENT-department and Audiological Centre in the Maastricht University Medical Centre (MUMC+). After living in Maastricht (the Netherlands) for one year, she now lives in Hasselt (Belgium) happily together with Gerd Cuppens, who became her husband in 2015, and their daughter Marlo Cuppens (2017). After five years of pure research work, she now combines her research activities at MUMC+ with clinical work as a Master Audiologist at the Audiological Centre of Adelante (Hoensbroek, the Netherlands). As active member of MAaN (Master Audiologists active in the Netherlands) she represents the interests of Master Audiologists working in Audiological Centres across the Netherlands.

\section{BIOGRAFIE}

Elke (Maria Johanna) Devocht werd geboren op 11 maart 1987 in Turnhout (België), waar ze opgroeide als oudste dochter in een warm en liefdevol gezin. Ze volgde haar kleuter, lager en middelbaar onderwijs binnen het Heilig Graf Instituut in Turnhout (België). In 2005 rondde ze met succes het algemeen secundair onderwijs af binnen de studierichting Latijn-Wiskunde en startte ze haar studies in de Logopedische- en Audiologische Wetenschappen aan de Katholieke Universiteit van Leuven (KUL, België). Ze behaalde haar Bachelor en vervolgens haar Master diploma met grootste onderscheiding in 2008 en 2009. Tijdens haar Master jaar koos ze voor de optie Audiologie waarvoor ze stage liep in het Audiologisch 
Centrum van het Amsterdam Medisch Centrum (AMC, Amsterdam, Nederland) en een gezamenlijke thesis schreef met als titel 'Lokalisatie en spatiaal spraakverstaan bij cochleair geïmplanteerde kinderen'. In 2010 besloot ze haar studies aan de KUL met een post graduaat in de Audiologie en Hoortoestel Aanpassing. In 2009 keerde ze reeds terug naar het Audiologisch Centrum van het AMC om daar haar loopbaan als Master Audioloog te starten. Gedurende drie jaar woonde ze in Weesp (Nederland) en deed ze waardevolle klinische ervaring op betreffende de aanpassing van cochlear implantaten en hoortoestellen binnen het volwassen gehoorrevalidatie team in het AMC. In 2012 nam ze de uitdaging aan om een promotietraject te starten verbonden aan de KNO afdeling en het Audiologisch Centrum van het Maastricht Universitair Medisch Centrum (MUMC+). Na een jaar in Maastricht (Nederland) gewoond te hebben, woont ze nu in Hasselt (België) gelukkig samen met Gerd Cuppens, haar echtgenoot sinds 2015, en haar dochtertje Marlo Cuppens (2017). Na vijf jaar zuiver wetenschappelijk onderzoek, combineert ze nu haar onderzoeksactiviteiten in het MUMC+ met klinische taken als Master Audioloog binnen het Audiologisch Centrum van Adelante (Hoensbroek, Nederland). Als actief lid van de MAaN (Master Audiologen actief in Nederland) vertegenwoordigt ze de belangen van Master Audiologen werkzaam binnen Audiologische Centra in Nederland.

\section{PUBLICATION LIST}

1. Devocht EMJ, George ELJ, Janssen AML, Stokroos RJ. Bimodal Hearing Aid Retention after Unilateral Cochlear Implantation. Audiol Neurotol. 2015;20: 383-393. doi:10.1159/000439344.

2. Devocht EMJ, Dees G, Arts RAGJ, Smits JJ, George ELJ, van Hoof M, et al. Revisiting PlacePitch Match in CI Recipients Using 3D Imaging Analysis. Ann Otol Rhinol Laryngol. 2016;125: 378-384. doi:10.1177/0003489415616130.

3. Devocht EMJ, Janssen AML, Chalupper J, Stokroos RJ, George ELJ. Monaural Beamforming in Bimodal Cochlear Implant Users: Effect of (A)symmetric Directivity and Noise Type. PLoS One. 2016;11: e0160829. doi:10.1371/journal.pone.0160829.

4. Devocht EMJ, Janssen AML, Chalupper J, Stokroos RJ, George ELJ. The Benefits of Bimodal Aiding on Extended Dimensions of Speech Perception : Intelligibility , Listening Effort, and Sound Quality. Trends Hear. 2017;20. doi:10.1177/2331216517727900.

5. Devocht EMJ, Janssen AML, Chalupper J, Stokroos RJ, Kingma H, George ELJ. Selfassessment of unilateral and bimodal cochlear implant experiences in daily life. Int. J. Audiol. 2019; Under review. 


\section{CO-AUTHORS AND AFFLLIATIONS}

ARTS, REMO A. G. J., DRS.

- Maastricht University Medical Center, School for Mental Health and Neuroscience (MHENS), Department of ENT/Audiology, Maastricht, The Netherlands.

CHALUPPER, JOSEF, DR.

- Advanced Bionics Inc., European Research Centre, Hannover, Germany.

DEES, GUIDO, DRS.

- Maastricht University Medical Center, School for Mental Health and Neuroscience (MHENS), Department of ENT/Audiology, Maastricht, The Netherlands.

GEORGE, ERWIN L. J., DR.

- Maastricht University Medical Center, School for Mental Health and Neuroscience (MHENS), Department of ENT/Audiology, Maastricht, The Netherlands.

HOOF VAN, MARC, DRS.

- Maastricht University Medical Center, School for Mental Health and Neuroscience (MHENS), Department of ENT/Audiology, Maastricht, The Netherlands.

JANSSEN, A. MIRANDA L., DRS.

- Maastricht University Medical Center, School for Mental Health and Neuroscience (MHENS), Department of ENT/Audiology, Maastricht, The Netherlands.

- Maastricht University, School for Public Health and Primary Care (CAPHRI), Department of Methodology and Statistics, Maastricht, The Netherlands.

KINGMA, HERMAN, PROF. DR.

- Maastricht University Medical Center, , School for Oncology and Develop Biology (GROW), Department of ENT, Maastricht, The Netherlands.

SMITS, JEROEN J., BSC

- Maastricht University Medical Center, School for Mental Health and Neuroscience (MHENS), Department of ENT/Audiology, Maastricht, The Netherlands.

STOKROOS, ROBERT J. , PROF. DR.

- Maastricht University Medical Center, School for Mental Health and Neuroscience (MHENS), Department of ENT/Audiology, Maastricht, The Netherlands.

- University Medical Center Utrecht, Department of ENT, Utrecht, The Netherlands.

- University Medical Center Utrecht, Brain Center Rudolf Magnus, Utrecht, The Netherlands. 
STELLINGEN HOREND BIJ HET PROEFSCHRIFT

\title{
COMBINING A COCHLEARIMPLANT AND
}

A HEARING AID IN OPPOSITEEARS

THE BEST OFBOTHWWORLDS

\author{
Elke Devocht \\ Maastricht, 3 juli 2019
}

1. De meerderheid van de mensen die in één oor een cochleair implantaat krijgen, kiezen er tegenwoordig voor om in het andere oor een akoestisch hoortoestel te blijven dragen. (dit proefschrift)

2. De resterende functionele prestaties met het hoortoestel in vergelijking met het resultaat van het cochleair implantaat maken dat men ervoor kiest om de bimodale combinatie te gebruiken. (dit proefschrift)

3. Bimodaal horen levert voordelen op voor de spraakperceptie, zowel op het vlak van vêrbeterd "spraakverstâan" als" verminderde" lưisterinspânning" "en "een vollere gêluidșkwaliteit. (dit proefschrift)

4. Nadere samenwerking en afstemming tussen elektrisch en akoestisch horen kunnen de complementariteit van beide werelden verder vergroten. (dit proefschrift)

5. De uitdaging binnen de hoorzorg bestaat uit het bepalen en afleveren van de meest efficiënte hooroplossing voor elk individu. (dit proefschrift en valorisatie)

6. Het cochleair implantaat is het eerste door de mensheid geproduceerd apparaat dat één van de menselijke zintuigen effectief herstelt. (Prof. G. Nossal)

7. Het belang van "communicatie voor dê menselijke sâmenleving "wordt al duizenden jaren erkend, veel lânger dan men kan àantonen in de geschiedenisboeken. (Richmond \& McCroskey, 2009)

8. Gehoorverlies zorgt niet enkel voor invaliderende sociale beperkingen maar vormt ook een significante kostenpost voor ơnzè maảtschappij. (Olüsanya et al, 2014)

9. Een cursus 'grensarbeid' kan niet ontbreken in het curriculum van een Master Audioloog.

10. Kijken naar kunst is als kijken door de ogen van een kind. (Jan Hoet jr.) 


\section{ABSTRACT}

Bimodal hearing: a cochlear implant in one ear and an acoustic hearing aid in the other. Many advantages, but also a lot of challenges. Who opts to use a bimodal fitting? What are the benefits? How does it support the perception of speech? Can this be improved by directional microphone systems? How can imaging help to approach natural hearing? Discover through this research how the best of both worlds is combined. 